The molecular biology of matrix metalloproteinases and tissue inhibitors of metalloproteinases in inflammatory bowel diseases

Peer-reviewed author version

de Bruyn, Magali; Vandooren, Jennifer; Ugarte-Berzal, Estefania; ARIJS, Ingrid; Vermeire, Severine \& OPDENAKKER, Ghislain (2016) The molecular biology of matrix metalloproteinases and tissue inhibitors of metalloproteinases in inflammatory bowel diseases. In: CRITICAL REVIEWS IN BIOCHEMISTRY AND MOLECULAR BIOLOGY, 51(5), p. 295-358.

DOI: 10.1080/10409238.2016.1199535

Handle: http://hdl.handle.net/1942/22827 


\section{The molecular biology of matrix metalloproteinases and tissue inhibitors of metalloproteinases in inflammatory bowel diseases.}

de Bruyn, Magali ${ }^{1,2}$; Vandooren, Jennifer ${ }^{1}$; Ugarte-Berzal, Estefi ${ }^{1}$; Arijs, Ingrid ${ }^{2,3}$; Vermeire, Séverine $^{2,4}$ and Opdenakker, Ghislain ${ }^{1}$.

1. Rega Institute for Medical Research, Laboratory of Immunobiology, Department of Microbiology and Immunology, KU Leuven, Leuven, Belgium. 2. Translational Research Center for Gastrointestinal Disorders (TARGID), Department of Clinical and Experimental Medicine, KU Leuven, Leuven, Belgium. 3. Faculty of Medicine and Life Sciences, Hasselt University, Hasselt, Belgium. 4. University Hospitals Leuven, Leuven, Belgium.

\section{Corresponding author:}

Prof. Dr. Ghislain Opdenakker

Rega Institute for Medical Research, Minderbroedersstraat 10 blok x - bus 1030, 3000 Leuven, Belgium.

Telephone number: +3216337341

Fax number: +3216337340

Email: ghislain.opdenakker@kuleuven.be

KEY WORDS: MMP, TIMP, IBD, association studies, interaction, inflammation, innate immune defense, adaptive immunity, remnant epitopes, autoimmunity, neutrophils, protease web, network hypothesis, degradome. 


\section{TABLE OF CONTENTS}

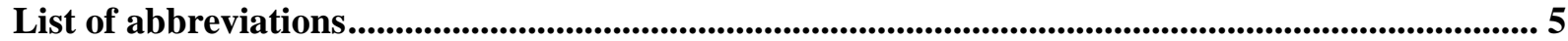

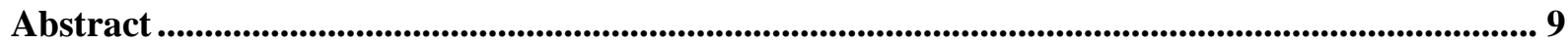

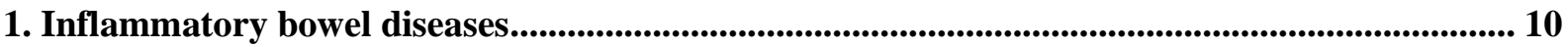

1.1 Epidemiology

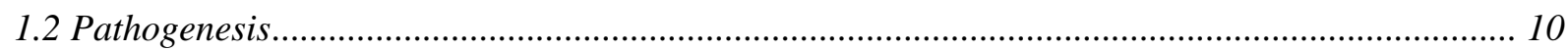

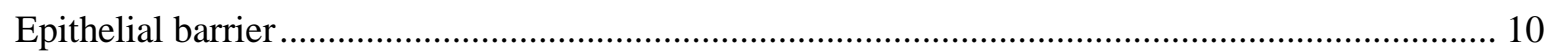

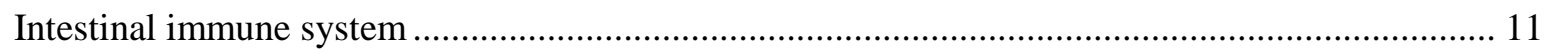

Gut microbiota

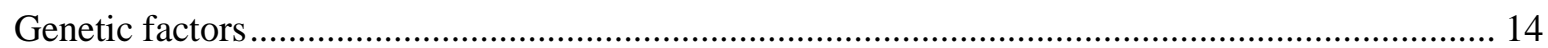

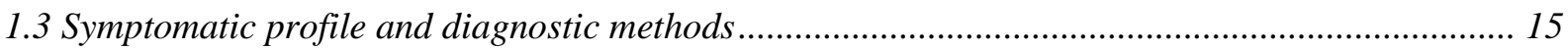

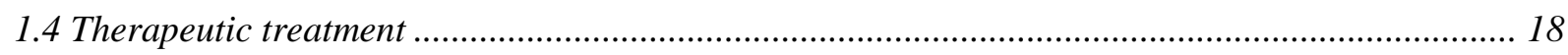

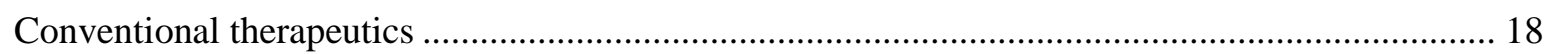

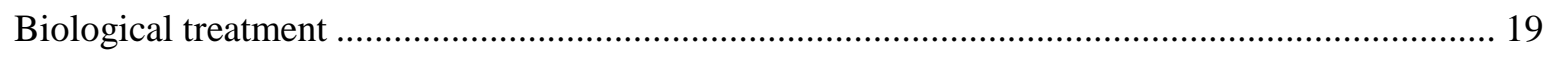

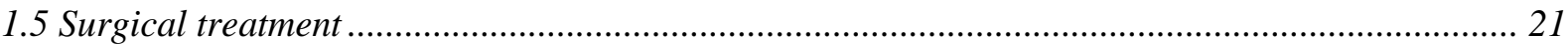

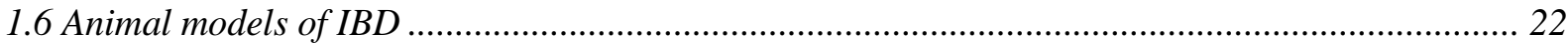

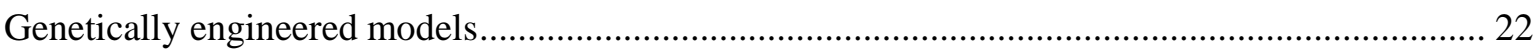

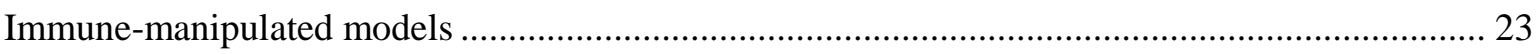

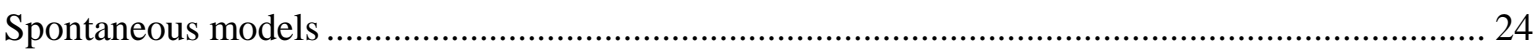

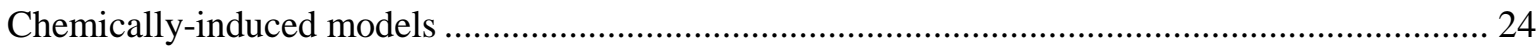

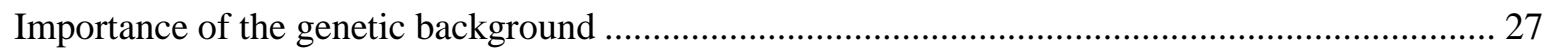

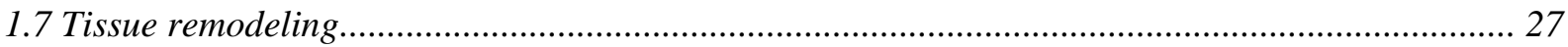

2. Interactions and balances between matrix metalloproteinases and tissue inhibitors of

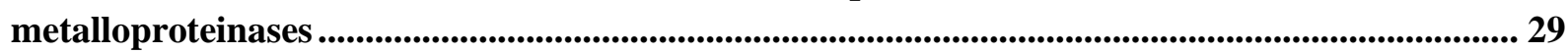

2.1 Matrix metalloproteinases (MMPs)

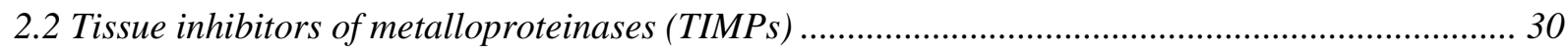

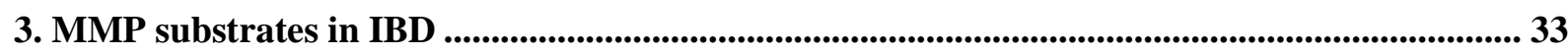

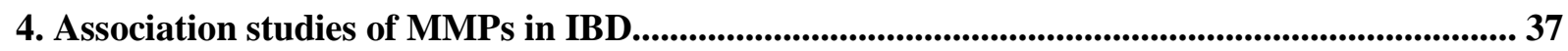

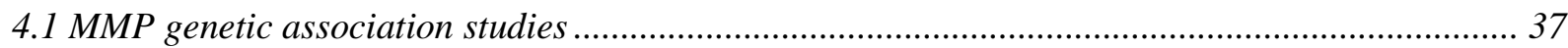

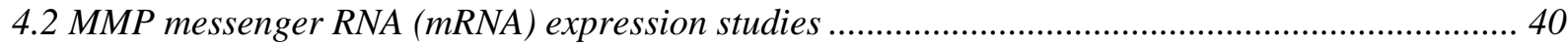

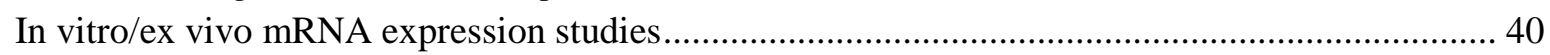

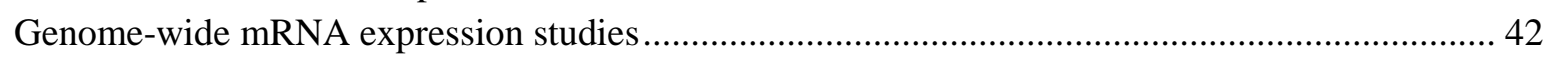

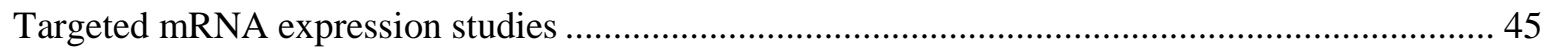

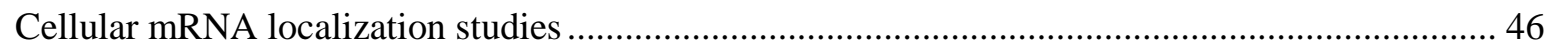

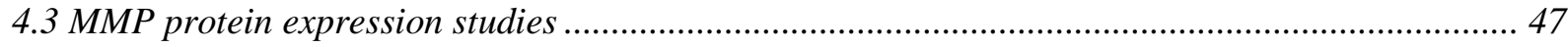

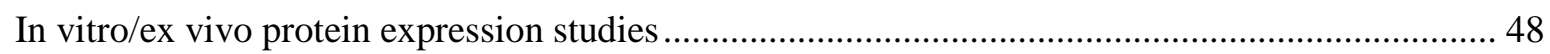

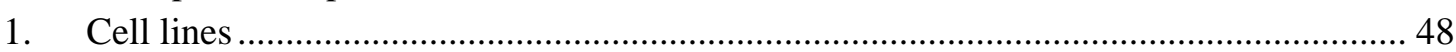

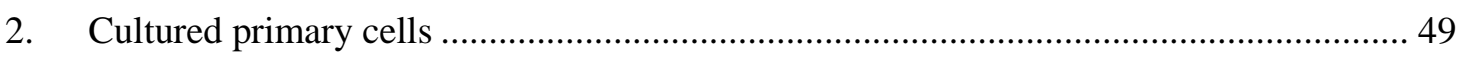




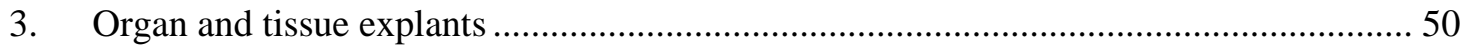

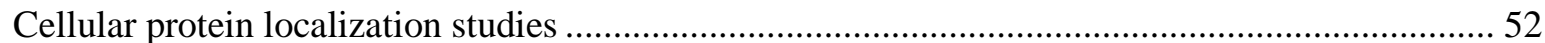

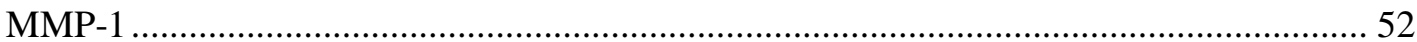

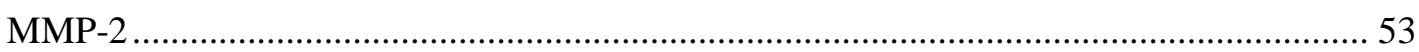

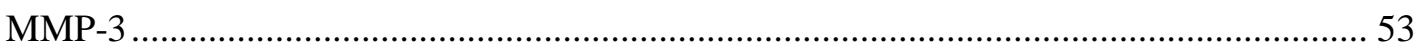

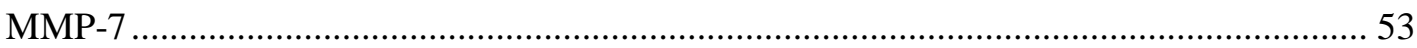

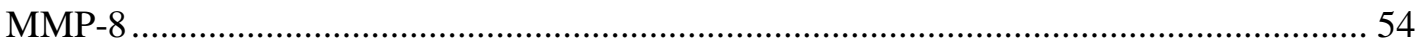

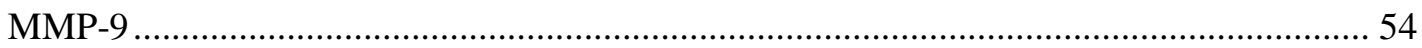

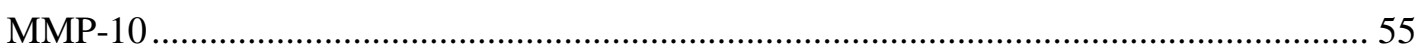

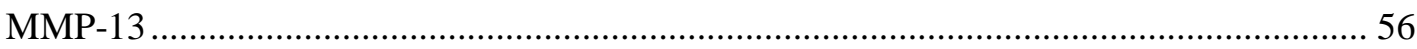

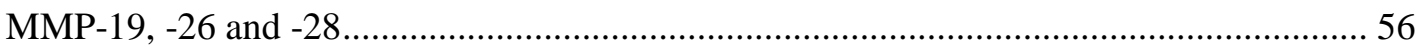

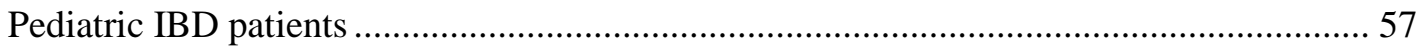

Changes in MMP expression and/or localization after treatment ...................................... 57

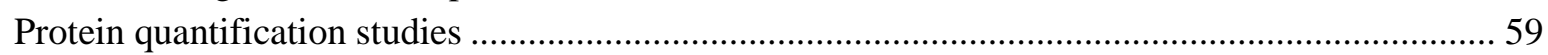

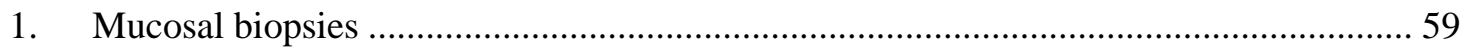

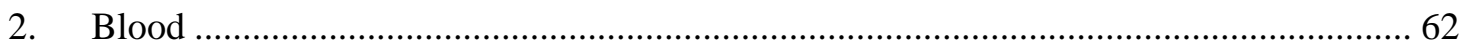

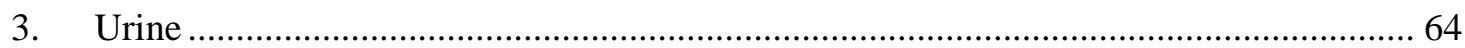

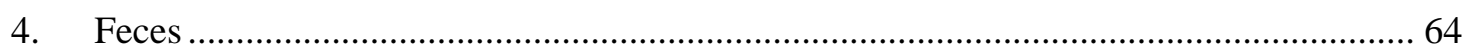

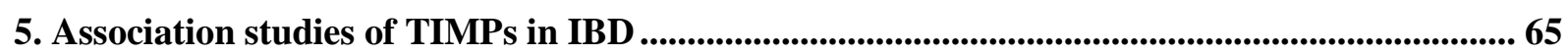

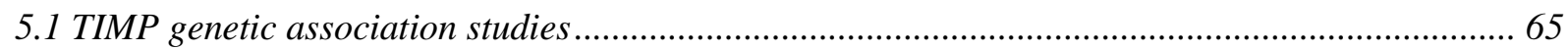

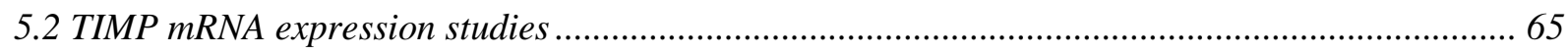

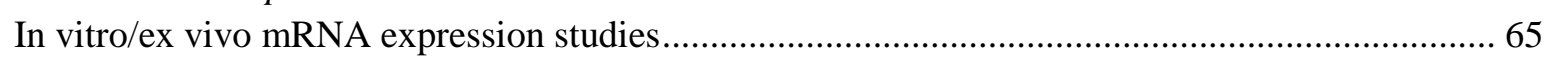

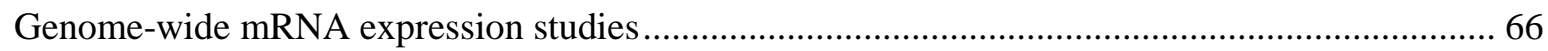

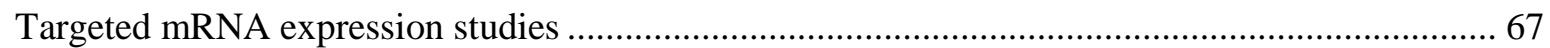

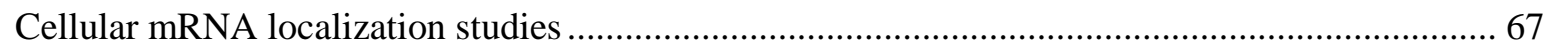

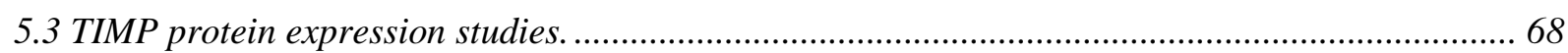

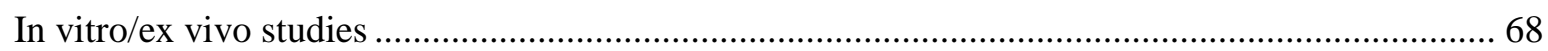

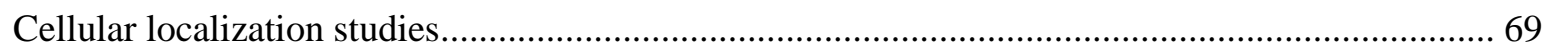

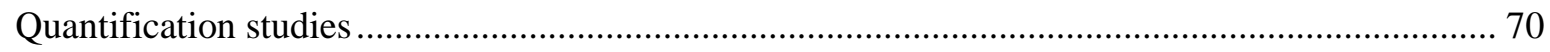

6. Animal model studies of MMPs and TIMPs ........................................................................................ 72

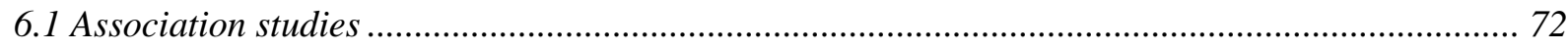

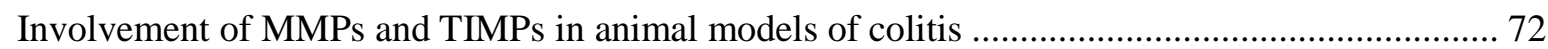

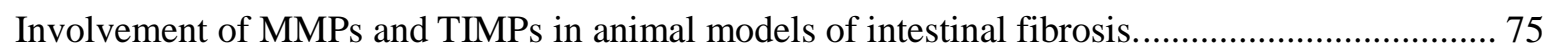

Effect of therapeutic intervention on MMPs and TIMPs in experimental colitis models. ............... 76

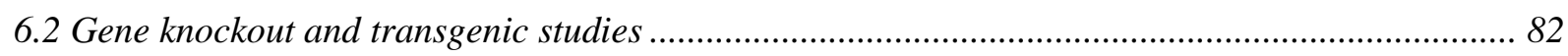

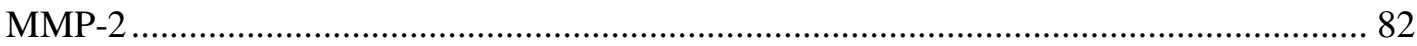

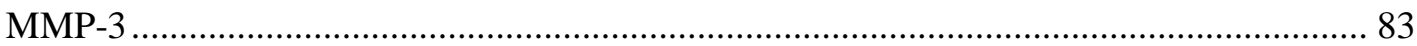

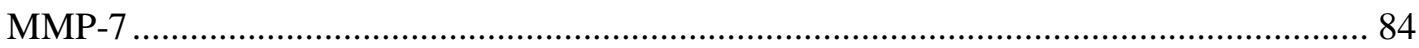

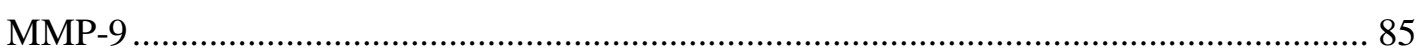

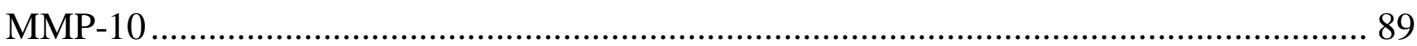

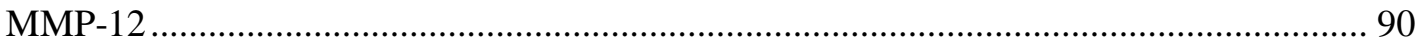

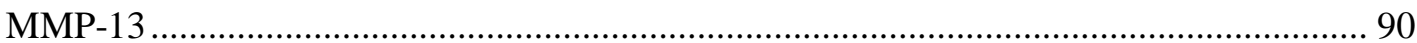

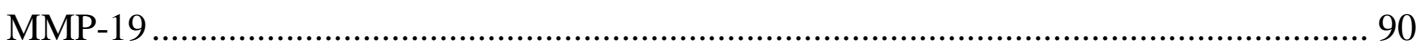

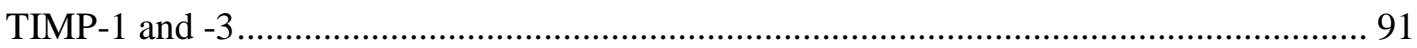


7. Studies with MMP inhibitors (MMPIs) ........................................................................................................ 92

8. Evidences for MMP inhibition as therapy for IBD in humans ....................................................... 95

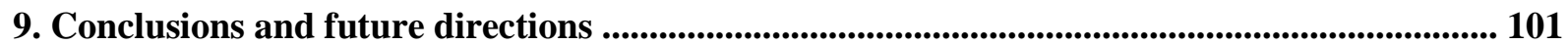

Acknowledgements ..................................................................................................................................... 105

Declaration of interest ..................................................................................................................................... 105

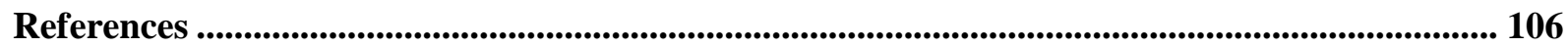

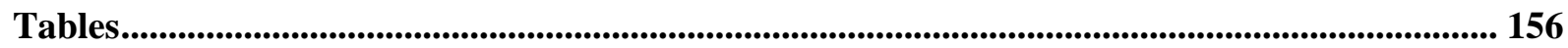

Figure legends .......................................................................................................................................................... 200 


\section{LIST OF ABBREVIATIONS}

5-ASA

6-MP

ALK5

AMP

ARE

ASCA

ATG16L1

AZA

BIA

CARD15

$\mathrm{CC}$

CD

$\mathrm{CDc}$

CDEIS

CDi

$\mathrm{CM}$

CRP

CS

DNA

DSS

DTH

ECM

ELISA

EPA

ESR

FAP 5-aminosalicylic acid

6-mercaptopurine

activin receptor-like kinase 5

antimicrobial peptide

AU rich elements

antibodies to Saccharomyces cerevisiae

autophagy related 16-like 1

azathioprine

biochemical immunosorbent activity assays

caspase recruitment domain-containing protein 15

collagenous colitis

Crohn's disease

Crohn's colitis

Crohn's disease endoscopic index of severity

Crohn's ileitis

Cordyceps militaris

C-reactive protein

corticosteroids

deoxyribonucleic acid

dextran sodium sulphate

delayed-type hypersensitivity

extracellular matrix

enzyme-linked immunosorbent assay

erythroid potentiating activity

erythrocyte sedimentation rate

familial adenomatous polyposis 
FDA

GC

GI

GPI

GWAS

$\mathrm{HC}$

HLA

IBD

IBS

ICAM-1

IEC

IFN

IL

IM

iNOS

IRGM

ISH

JAK

$\mathrm{Ki}$

$\mathrm{KO}$

LA

LC

LCN2

LPMC

LPS

MadCAM-1

MDD
Food and Drug Administration

glucocorticoid

gastrointestinal

glycosyl-phosphatidyl-inositol

genome-wide association study

healthy control

human leukocyte antigen

inflammatory bowel disease

irritable bowel disease

intercellular adhesion molecule 1

intestinal epithelial cell

interferon

interleukin

irsogladine maleate

inducible nitric oxide synthase

immunity-related GTPase family, M

in situ hybridization

Janus kinase

inhibitory constant

knock-out

$\alpha$-lipoic acid

lymphocytic colitis

lipocalin-2

lamina propria mononuclear cell

lipopolysaccharide

mucosal vascular addressin cell adhesion molecule 1

methyl-deficient diet 
macrophage migration inhibitory factor

MLCK

myosin light chain kinase

MMP

matrix metalloproteinase

MMPI

matrix metalloproteinase inhibitor

MPO

myeloperoxidase

mRNA

messenger RNA

MSC

mesenchymal stem cell

MSS

musculoskeletal syndrome

MTX

methotrexate

MW

molecular weight

NGAL

neutrophil gelatinase B-associated lipocalin

NGAL-MMP-9

neutrophil gelatinase B-associated lipocalin and matrix metalloproteinase-9 con

NOD

nucleotide oligomerization domain

Omp C

outer membrane protein $\mathrm{C}$

pANCA

perinuclear antineutrophil cytoplasmic antibodies

PAP

protease-activated prodrugs

PDE4

phosphodiesterase 4

PE

prolyl endopeptidase

PGP

proline-glycine-proline

PHA

polyhydroxyalkanoates

PMNL

polymorphonuclear leukocytes

PNR

primary non-responder

PPAR- $\gamma$

proliferator-activated receptor- $\gamma$

PPG

phenylpropanoid glycosides

PSC

primary sclerosing cholangitis

qRT-PCR

quantitative reverse transcription polymerase chain reaction

RNA

ribonucleic acid 
soluble

S.T.

SAMP1

SCID

sFASL

siRNA

SIRT

SNP

SPF

$\mathrm{SSH}$

STAT

T reg

TACE

TGF

Th

TIMP

$\mathrm{Tm}$

TNBS

TNF

UC

UCMSC

VCAM

VEGF

VEGFR

VIP

WT
Salmonella enterica subsp. serovar typhimurium

senescence accelerated mouse prone 1

severe combined immunodeficiency

soluble Fas ligand

small interfering RNA

silent information regulator

single nucleotide polymorphism

specific pathogen free

subtractive suppression hybridization

signal transducer and activator of transcription

$\mathrm{T}$ regulatory

TNF-alpha converting enzyme

transforming growth factor

T helper

tissue inhibitor of metalloproteinases

transmembrane

trinitrobenzenesulfonic acid

tumor necrosis factor

ulcerative colitis

umbilical cord mesenchymal stem cell

vascular cell adhesion molecule

vascular endothelial growth factor

vascular endothelial growth factor receptor

vasoactive intestinal peptide

wild-type 


\section{ABSTRACT}

Matrix metalloproteinases (MMPs) and tissue inhibitors of metalloproteinases (TIMPs) are thought to be predominant proteases and protease inhibitors involved in the pathogenesis of inflammatory bowel diseases (IBD) through their ability to remodel the extracellular matrix (ECM) in response to inflammatory stimuli and by their immunomodulating effects. An imbalance between MMPs and TIMPs has been linked with acute and chronic inflammation and aberrant tissue remodeling, as seen in IBD. Moreover, recurrent phases of tissue destruction and subsequent tissue repair can cause serious complications in IBD patients such as fistulas and fibrosis. The aims of this review are (i) to summarize current literature on genetic association, mRNA and protein expression studies with regard to MMPs and TIMPs in IBD patients and various animal models, including those with transgenic and knockout mice; (ii) to compare biochemical and molecular biological data in humans with those obtained in animal model studies and (iii) to critically evaluate and translate how this knowledge may be used in practical terms to understand better the pathophysiology and mechanisms operating in IBD and to apply this for improvement of clinical outcomes at diagnostic, prognostic and therapeutic levels. 


\section{INFLAMMATORY BOWEL DISEASES}

\subsection{Epidemiology}

Inflammatory bowel diseases (IBD), including ulcerative colitis (UC) and Crohn's disease (CD), are chronic disabling diseases of the gastrointestinal tract that mostly affect young adults. The incidence of UC was shown to be the highest in Europe, whereas more CD patients are newly diagnosed in North-America. Although data are scarce for developing countries, it is clear that the incidence and prevalence of IBD are increasing worldwide (Kaplan, 2015; Molodecky et al, 2012). Environmental factors play a pivotal role in the increasing incidence around the world (Danese et al, 2004). The strongest environmental factors identified are cigarette smoking and appendectomy. Nicotine use increases the risk of CD and worsens the disease course, whereas it has a protective effect in UC. Appendectomy is thought to lower the risk of developing $\mathrm{UC}$, whereas it increases the risk of developing $\mathrm{CD}$. Whether other factors such as diet, oral contraceptives, perinatal/childhood infections, or atypical mycobacterial infections play a role in the development of IBD remains unclear (Loftus, 2004).

\subsection{Pathogenesis}

Although the etiology of IBD has been extensively studied, the disease pathogenesis is not fully understood. It is thought that an abnormal immune response is elicited towards the (normal) luminal microbiota in a genetically susceptible host.

\section{Epithelial barrier}

The intestinal epithelium provides an interface between the microbiome and the lymphoid tissue of the gastrointestinal system and is important for shaping the mucosal immune response. The luminal surface of the human intestine is lined with intestinal epithelial cells (IEC) (Sun et al, 2015). Intercellular junctions are needed to seal the gaps between adjacent IECs (the paracellular space), whereas tight junction complexes including claudin, occludin, E-cadherin 
and zonulin-1 are the key elements to form a physical barrier against pathogens penetrating the lamina propria. Moreover, IECs provide an immune defense function by transporting immunoglobulin A and antimicrobial proteins (AMPs) from their cellular sources to the intestinal lumen. Besides IECs, more specialized epithelial cells also play an important role in maintaining the intestinal barrier. Goblet cells regulate the formation of a mucus layer and produce factors needed for epithelial repair and regulation of inflammation. The importance of the mucus layer was emphasized by studies demonstrating that MUC2 knock-out (KO) mice had no bacteria-free zone and developed spontaneously intestinal inflammation (Van der Sluis et al, 2006). Paneth cells are located at the base of crypts and secrete AMPs such as $\alpha$-defensins (Abraham \& Cho, 2009). In IBD, many of these first-line defense mechanisms are dysregulated. The paracellular space has an increased permeability and tight junction complexes can be cleaved by bacterial proteases leading to a breach in the intestinal barrier (Pruteanu \& Shanahan, 2013; Turner, 2006). The secretion of defensins is lowered in the ileum and the mucus layer is thinner due to loss of mucus-secreting goblet cells.

\section{Intestinal immune system}

Since the gastrointestinal tract is exposed to an extraordinarily high antigen load, the intestinal immune system requires a fine balance between a state of tolerance towards intestinal antigens and the ability to combat pathogens. Both innate and adaptive immune cells are important to maintain this balance. Innate immune cells include neutrophils, macrophages, dendritic cells and natural killer cells. They provide an initial, rapid response towards invading pathogens by recognizing pathogen-associated microbial patterns with their pattern-recognition receptors. Neutrophils are the first responders of the innate immune system and their primary function is to kill and digest bacteria via phagocytosis and digestion, and through secretion of digestive enzymes. Infiltration of neutrophils in the lamina propria is a hallmark of IBD and the degree of neutrophil migration into intestinal crypts correlates with patient symptoms and mucosal 
injury (Brazil et al, 2013). Neutrophils directly influence intestinal homeostasis by disturbing the permeability of the epithelial barrier. Moreover, the secretion of cytotoxic mediators such as hydrogen peroxide and hydroxyl radicals by neutrophils is related to subsequent tissue damage (Kucharzik et al, 2001). Macrophages have phagocytic properties, but have less killing and less digestive capacity compared to neutrophils. They form granulomata to shield pathogenic material from the rest of the body and secrete cytokines that prime and amplify the immune response. Dendritic cells are a specialized type of antigen presenting cells that are able to link the innate with the adaptive immunity. After encountering pathogens, dendritic cells present peptide antigens to T cells in secondary lymphoid organs in the gut (e.g. Peyer's patches, mesenteric lymph nodes and isolated lymphoid follicles) and thereby initiate the adaptive immune response.

For many years it was assumed that the adaptive immune response in UC was mediated by $\mathrm{T}$ helper (Th) 2 cells, whereas the response in CD was mediated by Th1 cells. This was supported by reports of increased levels of Th1 cytokines such as IFN- $\gamma$ and IL-12 in CD and an increased expression of IL-13 in UC (Berrebi et al, 1998; Brand, 2009; Heller et al, 2005; Parronchi et al, 1997). With the discovery of other T cell populations, it was found that T regulatory (Treg) cells and Th17 cells also play an important role in IBD (Allez \& Mayer, 2004; Brand, 2009; Kitani \& Xu, 2008). Treg cells are important mediators of immune responses towards selfantigens and Th17 cells were recognized as a group of T cells that produce IL-17. Both cell types play a major role in autoimmunity.

\section{Gut microbiota}

The intestinal epithelium and the host immune system are in constant contact with the gut microbiota. Trillions of microbes are present in the gut and they develop a symbiotic bond with the immune system. The composition of the gut microbiota can regulate the adaptive immune response by influencing the balance between effector and regulatory $\mathrm{T}$ cells (Kuchroo et al, 
2012). Moreover, the presence or absence of a single species can have a major effect on the immune system and can disturb this balance (Ivanov et al, 2009; Mohamadzadeh et al, 2011; Round et al, 2011). In IBD, an imbalance in the composition of the gut microbiota, termed dysbiosis, has been determined to play an important role in the disease pathogenesis (Becker et al, 2015; Kamada et al, 2013; Round \& Mazmanian, 2009; Sartor, 2008). In general, the gut flora show a decreased diversity of microbial species and are more unstable over time in patients with IBD compared to individuals with a healthy gut (Andoh et al, 2012). Confirmed reported changes are a reduction in Firmicutes and Bacteroides, and an increase in Actinobacteria and Proteobacteria (Frank et al, 2007; Morgan et al, 2012).

Evidence for a pivotal role of microbiota in the pathogenesis of IBD comes from various routes. Recurrence of $\mathrm{CD}$ in the neoterminal ileum after curative ileal resection was shown to be dependent on the presence of the fecal stream, since patients who had surgery without restoration of the fecal stream did not develop endoscopic lesions (Rutgeerts et al, 1991). The important role of gut microbiota in IBD is further underlined by animal studies. Animal strains in which spontaneous chronic colitis occurs (e.g. Human Leukocyte Antigen (HLA) B27 transgenic rats and IL-10 or IL-2 deficient mice) seem to be dependent on the presence of luminal flora (Round \& Mazmanian, 2009; Wirtz \& Neurath, 2007). Intestinal inflammation in these strains occurs only in conventional conditions and not when the animals are raised under germ-free conditions. Moreover, colitis was induced in murine strains that were kept in a gnotobiotic state by reconstitution of bacteria which are considered normal constituents of the luminal flora (Elson et al, 2005). Transfer of microbiota from mice that developed colitis to a healthy mouse resulted in intestinal inflammation (Garrett et al, 2007) as did the transfer of T cells that were shown to be reactive against commensal organisms (Kullberg et al, 2003). Most of the studies investigating the gut flora in IBD are focused on determining bacterial species, whereas only a few studies addressed the role of viral components (virome) and micro- 
eukaryotes (protozoa and fungi). Recently, it was shown that disease-specific alterations occur in the enteric virome of IBD patients with a significant expansion of Caudovirales bacteriophages (Norman et al, 2015).

\section{Genetic factors}

Further highlighting the importance of host-microbe interactions, meta-analysis of genomewide association studies (GWAS) and Immunochip data identified 201 genetic susceptibility loci in IBD (Abraham \& Cho, 2009; Cho, 2008; Cleynen et al, 2013; Jostins et al, 2012; Liu et al, 2015). Many of these genes are associated with mucosal barrier function, bacterial sensing, ER stress, autophagy and immune regulation. Some of these identified loci are allocated uniquely to $\mathrm{UC}$ or $\mathrm{CD}$, whereas others have been shown to occur in both disease types (Lees et al, 2011). Nucleotide-binding oligomerization domain 2 (NOD2) was the first gene locus to be identified as a susceptibility gene in CD. It encodes an intracellular receptor that recognizes the bacterial peptidoglycan component muramyl dipeptide and therefore plays an important role in bacterial sensing. Mutations in NOD2 were linked to a defective innate immune response which has been linked - in part - with a lowered secretion of $\alpha$-defensins (Tan et al, 2015). Alterations in genes playing a role in bacterial killing (e.g. autophagy-related 16-like protein 1 (ATG16L1) and immunity-related GTPase family $\mathrm{M}(I R G M))$ have also been described in CD patients. ATG16L1 is involved in the degradation of intracellular pathogens and ATG16L1 deficiency has been associated with defective Paneth cell function (Cadwell et al, 2008). IRGM plays a role in the control of the intracellular mycobacterial load since mice deficient in IRGM have an impaired ability to eliminate intracellular pathogens (e.g. Toxoplamsa gondii and Listeria monocytogenes) (Collazo et al, 2001). The associations of single nucleotide polymorphisms (SNPs) in NOD2, ATG16L1 and IRGM have only been described for CD and not for UC. This indicates that deficiencies in bacterial sensing and killing are a key feature of the pathogenesis of CD. In contrast, components of the IL-23R pathway were identified as genetic risk factors in 
both CD and UC patients (Cho, 2008). IL-23 signaling is mediated by binding of heterodimeric IL-23 (p19 and p40 subunits) to the heterodimeric receptor (containing IL-23R and IL-12RB1). This results in activation of the Janus Kinase - Signal Transducer and Activator of Transcription (JAK-STAT) pathway, whereby STAT3 and STAT4 play important roles in Th1 and Th17 differentiation.

\subsection{Symptomatic profile and diagnostic methods}

The symptomatic profile of UC and CD is quite similar. Patients can experience bloody diarrhea, passage of pus/mucus, abdominal cramping, fever and fatigue, loss of appetite and unintended weight loss. The severity of clinical symptoms in UC patients is related to the extent and activity of the disease. In general, diffuse mucosal inflammation is observed in UC which is restricted to the colon and extends proximally from the rectum. Patients can have inflammation only in the rectum (proctitis), in the sigmoid colon with or without the descending colon (left-sided colitis) or in the entire colon (pancolitis). CD patients present with patchy, transmural inflammation that can affect the entire gastrointestinal (GI) tract and can be classified according to the age at diagnosis and the location and behavior of disease. In half of the patients, the disease is located at the terminal ileum at time of diagnosis, whereas patients with only colonic or only ileal disease are less frequent. Few patients display upper GI inflammation. Many CD patients suffer from complications e.g. fibrotic strictures, fistulas and abscesses, whereas some have perianal disease.

Besides differences in clinical manifestation, an endoscopic evaluation is needed to accurately diagnose patients and to objectively assess the disease location and activity in patients. UC is characterized by loss of normal vascular pattern, by erythema, granularity, ulceration and spontaneous bleeding. In CD, patchy lesions including aphthoid ulceration or larger deep ulcers alternate with normally looking mucosa. A cobblestone pattern of the mucosa is mostly seen in later stages of the disease (Figure 1). During an endoscopic evaluation, mucosal biopsies can 
be taken from the bowel and used for subsequent histopathological analysis. Microscopic features of $\mathrm{CD}$ include infiltration of inflammatory cells in the lamina propria and presence of granuloma with giant cells (Figure 1). Since biopsies are taken from the mucosal layer and CD is a transmural disease, it is difficult to fully assess disease activity in CD patients with histopathological analyses. In UC, infiltration of inflammatory cells into the lamina propria, goblet cell depletion, crypt abscesses and epithelial ulceration can be observed in active disease (Figure 1).

In addition, a range of biomarkers have been identified to aid clinicians in diagnosis, disease activity assessment and determination of response to therapy. Perinuclear antineutrophil cytoplasmic antibodies (pANCAs) and antibodies to Saccharomyces cerevisiae antigens (ASCA) are two serological markers that can aid in the differential diagnosis of UC and CD. Usually, both markers are tested together to enhance the specificity and sensitivity of the test. Most patients with UC are pANCA positive and ASCA negative, whereas most patients with small intestinal CD are pANCA negative and ASCA positive. Other serological markers include E. coli outer membrane protein C (Omp C), Pseudomonas fluorescens (I2) and flagellins (CBir1, FlaX and Fla2).

C-reactive protein $(\mathrm{CRP})$ is an acute phase protein produced by hepatocytes and measured in the serum. Upon an inflammatory stimulus mediated by IL-6, TNF- $\alpha$ or IL- $1 \beta$, hepatocytes rapidly increase the production of CRP. The half-life of CRP is relatively short ( 19 hours) and therefore it is mainly a marker of acute inflammation. However, some discrepancies exist in the use of CRP as a marker for IBD. Not all UC or CD patients with active disease have elevated CRP levels. For UC, only half of the patients have elevated CRP levels (Tall, 2004), whereas this is the case for two thirds of the CD patients (Jurgens et al, 2011). Moreover, CRP is not specific for IBD since it is used as a general marker of inflammation in many diseases. Other 
peripheral venous blood markers include erythrocyte sedimentation rate (ESR), white blood cell count, platelet count and serum albumin (Iskandar \& Ciorba, 2012).

Fecal sampling may provide several advantages over serum sampling. It is less invasive and can provide more accurate information on actual elevation of mucosal inflammatory markers in the bowel. Calprotectin is a zinc- and calcium-binding protein stored in neutrophil granules. It is part of the S100 protein family as it forms a dimer between S100A8 and S100A9. Fecal calprotectin is a stable marker as it is resistant to bacterial degradation and it can be kept intact for one week at room temperature (Roseth et al, 1992). It represents more than $60 \%$ of the cytosolic proteins in granulocytes and is therefore considered directly proportional to the amount of migrated neutrophils into the gut. Fecal calprotectin is therefore considered a very sensitive marker of intestinal inflammation. However, it is not a specific marker since increased levels are also found in neoplasia, polyps, other types of IBD, increasing age and infections (Bennike et al, 2014). Another fecal marker is lactoferrin, an iron-binding glycoprotein also found in the granules of neutrophils (Kane et al, 2003). It has antimicrobial properties and is resistant to degradation and proteolysis (Mendoza \& Abreu, 2009). Fecal lactoferrin is a sensitive marker for intestinal inflammation. However, like calprotectin, it is not specific for IBD although it can distinguish IBD from irritable bowel syndrome (IBS).

S100A12 is a protein that belongs to the $\mathrm{S} 100$ protein family and has similar calcium-binding properties as fecal calprotectin. Elevated levels of S100A12 are detectable in both serum and feces, however, fecal S100A12 levels were shown to distinguish IBD from IBS patients with higher specificity and sensitivity (Kaiser et al, 2007; Manolakis et al, 2010). Recently, S100A12 levels were associated with response to anti-TNF treatment (Boschetti et al, 2015). Lipocalin2, also known as neutrophil gelatinase B-associated lipocalin (NGAL), is found in secondary granules of neutrophils (Kjeldsen et al, 1994) and can be expressed by a variety of other cell types in response to activation of Toll-like receptors during infections (Chan et al, 2009). It has 
antimicrobial properties via sequestration of iron-laden siderophores (Flo et al, 2004). An upregulation of mucosal NGAL mRNA levels was found in IBD patients with active disease (Arijs et al, 2009). Moreover, protein levels of NGAL were found to be elevated in both serum and feces of IBD patients and the levels correlated with disease activity (Chassaing et al, 2012; Nielsen et al, 1999; Oikonomou et al, 2012).

In conclusion, there is not one single biomarker that can currently be used to assess or predict diagnosis, disease activity or response to treatment with sufficient sensitivity and specificity. Therefore, clinicians rely on a multidisciplinary approach combining clinical assessment with endoscopic and histologic evaluation as well as monitoring the disease in patients with the use of a range of biomarkers.

\subsection{Therapeutic treatment}

\section{Conventional therapeutics}

5-aminosalicylic acid (5-ASA or mesalazine) is a salicylic acid derivative and acts as an antioxidant. It is used as a first-line therapy for patients with mild to moderate UC. Several oral and rectal therapies exist and both are effective for induction and maintenance of remission in UC (Feagan \& Macdonald, 2012a; b; Marshall et al, 2010; 2012). In contrast, little evidence has been provided that 5-ASA has the same efficacy in CD patients. Corticosteroids (CS) are frequently used in clinical practice since they exert a fast and consistent clinical benefit in both UC and CD patients. The effect of CS is mediated through interaction with glucocorticoid receptors and subsequent modulation of the immune response (Ford et al, 2011). In several clinical studies it was shown that CS are effective for induction of remission in UC and CD. However, they are not suited for maintaining remission due to toxicity and side-effects (Ford et al, 2011). Despite these concerns and the increasing use of immunomodulatory treatment, the used of CS has not yet diminished. Thiopurines (azathioprine [AZA] and 6-mercaptopurine [6MP]) exert their cytotoxic and immunosuppressive effects through incorporation of false purine 
analogs in DNA or RNA. This induces DNA damage, cell-cycle arrest and apoptosis which results in inhibition of inflammatory gene expression (Coskun et al, 2016; Thomas et al, 2005). AZA is a prodrug that requires conversion to 6-MP in the gut and liver to exert its biological activity. Thiopurines can be used to maintain remission in both CD and UC (Khan et al, 2011). They can also be used in the case of glucocorticoid dependence or resistance, after CD surgery and after failure of UC maintenance therapy with 5-ASA. Methotrexate (MTX) is a folic acid antagonist that has anti-inflammatory and pro-apoptotic properties by blocking dihydrofolate reductase, interfering with DNA synthesis and inhibiting de novo purine synthesis. MTX has been shown to induce and maintain remission in $\mathrm{CD}$ patients and can be given postoperatively. Several large clinical trials are nearing completion in the assessment of MTX efficacy in UC patients (Nielsen et al, 2015). To reduce the risk of potential side effects by MTX, it is highly advised to give patients folic acid supplementation (Saibeni et al, 2012).

\section{Biological treatment}

In the last 15 years, several antibodies directed against TNF have been developed (Danese et al, 2015). They target both soluble (s) and transmembrane (tm) TNF by blocking their binding to the TNF receptor which is expressed on several cell types (mainly monocytes, macrophages and lymphocytes). This dampens the cytokine production of the cells and causes these to go into apoptosis (ten Hove et al, 2002; van den Brande et al, 2005; Van den Brande et al, 2003). It is assumed that mainly the blocking of tmTNF, rather than STNF, is responsible for the observed beneficial effects (Horiuchi et al, 2010). Infliximab, a chimeric monoclonal IgG1 antibody, was the first to be approved for induction and maintenance of remission in CD patients and later also in UC patients (D'Haens et al, 1999; Hanauer et al, 2002; Present et al, 1999; Rutgeerts et al, 2005; Sands et al, 2004). Further specialized indications for infliximab are CD patients with fistulizing disease, pediatric CD patients and patients with extraintestinal manifestations (Van Assche et al, 2009). Adalimumab and golimumab are fully human IgG1 
monoclonal antibodies. The Fc region of these agents is capable of complement activation and Fc-receptor-mediated biologic activities. Several clinical trials have shown that adalimumab can be used for induction and maintenance of remission in UC and CD patients (Colombel et al, 2007; Hanauer et al, 2006; Reinisch et al, 2011; Sandborn et al, 2007; Sandborn et al, 2012). Golimumab is approved for treatment of UC patients (Sandborn et al, 2014). Additional biological functions of anti-TNF agents other than mere neutralization of TNF have been suggested (Mitoma et al, 2005). For infliximab in particular, it has been shown to induce a significant dose-dependent increase in tissue inhibitor of metalloproteinase-1 (TIMP-1) production, to enhance the migration of and decrease the collagen production by $\mathrm{CD}$ myofibroblasts (Di Sabatino et al, 2007). Moreover, infliximab also exerts a beneficial effect on intestinal epithelial cells leading to restoration of the gut barrier and has been shown to deactivate the mucosal endothelium and thereby inhibit continued $\mathrm{T}$ cell recruitment (Danese, 2008).

Despite the spectacular initial results, approximately $30 \%$ of patients does not respond to antiTNF treatment (primary non-responders, PNR) or loses response over time (secondary nonresponders). The mechanisms underlying PNR remain largely unknown (Yanai \& Hanauer, 2011). The problem of loss of response over time is linked to immunogenicity (Baert et al, 2003) and therapeutic drug levels (Vande Casteele et al, 2015). Recently, it was shown that combined immunosuppressive treatment of infliximab and AZA was able to induce higher remission rates than infliximab or AZA monotherapy (Colombel et al, 2010). This beneficial effect of combination therapy is likely to be exerted by complementary immunomodulatory mechanisms or by reducing the formation of neutralizing antibodies (Baert et al, 2003).

After the anti-TNF era, anti-adhesion molecules were created as a second generation of biologicals. Drugs targeting adhesion proteins were generated in order to block the infiltration of inflammatory cells into the site of gut inflammation. Natalizumab is a humanized IgG4 
monoclonal antibody directed against the $\alpha 4$ integrin adhesion molecule and was initially approved for the treatment of multiple sclerosis (Miller et al, 2003). Several studies indicated its efficiency for induction and maintenance of remission in CD patients. However, severe sideeffects (e.g. progressive multifocal leukoencephalopathy) led to the withdrawal of the drug by the Food and Drug Administration (FDA) for its use in IBD. Subsequently, gut-specific antiadhesion molecules were developed. Vedolizumab targets the $\alpha 4 \beta 7$ integrin which is almost uniquely expressed on gut-homing $\mathrm{T}$ cells and interacts with mucosal vascular addressin cell adhesion molecule 1 (MadCAM-1) on endothelial cells to block $\mathrm{T}$ cell migration into the inflamed gut. Vedolizumab has been recently approved for the induction and maintenance of remission in UC and CD patients (Feagan et al, 2013; Sandborn et al, 2013).

\subsection{Surgical treatment}

Up to 35\% of UC patients (Landy et al, 2012) and 80\% of CD patients (De Cruz et al, 2012) who are refractory to conventional treatment or develop complications (e.g. strictures and fistulas) eventually require surgical treatment. For UC patients the common procedure is a twostage proctocolectomy with ileal pouch-anal anastomosis (Hocevar \& Remzi, 2001), whereas for CD patients with inflammation in the terminal ileum and caecum an ileocaecal resection is performed. However, surgical treatment is not curative. Up to $50 \%$ of UC patients develop inflammation in the pouch mucosa (pouchitis) (Shen, 2012) and nearly $70 \%$ of CD patients require a second surgical resection (Frolkis et al, 2014; Van Assche \& Rutgeerts, 2004). Although biological therapy has led to reduced rates of intestinal resection over the past few decades, a significant proportion of IBD patients, especially patients with a fibrostenosing phenotype, still need surgery (Frolkis et al, 2013; Kaplan et al, 2012; Singh et al, 2015). 


\subsection{Animal models of IBD}

During the last half century, many animal models of intestinal inflammation have been developed and these were mainly based on chemical induction. However, it was only in the last 20 years with the advanced knowledge of immunology and genetics that a new generation of mouse models with chronic intestinal inflammation was developed (Hibi et al, 2002; Strober, 1985; Wirtz \& Neurath, 2000; 2007). IBD animal models represent a critical tool to study the immunopathology of IBD as well as to evaluate the performance of potential drugs (Valatas et al, 2013). There are 4 major categories of IBD mouse models (see below) and some of the most widely used models are summarized in Table 1. Despite the intensive research and the multitude of animal models, there is - unfortunately - not one single mouse model that fully represents all features of the human disease.

\section{Genetically engineered models}

One of the first models that changed the IBD mouse model field was the IL-10 knock-out mouse. IL-10 is produced by Treg cells and plays an important anti-inflammatory role. IL-10 deficient mice present with weight loss and anemia which are the result of an extensive enterocolitis that affects the entire gastrointestinal tract (Kuhn et al, 1993). The lesions seen in this model closely resemble those of CD patients and are mainly mediated by a Th1 inflammatory response. The IL-10 knock-out mouse has played an important role in unraveling the involvement of the microbiota in the pathogenesis of IBD. IL-10 deficient mice were shown to develop chronic enterocolitis under conventional housing conditions. However, when the mice were kept under specific pathogen-free (SPF) conditions, they developed a localized colitis and anemia, but not enteritis. Moreover, germ free IL-10 deficient mice did not develop enterocolitis unless specific bacteria were administered. A second model of genetic modification is the TNF overexpression model $\left(\mathrm{TNF}^{\triangle \mathrm{ARE}}\right)$. In this model, AU-rich elements (ARE), which are present in the 3' untranslated region and responsible for repressing translation 
of $\mathrm{TNF}$, were deleted. As a result, $\mathrm{TNF}^{\triangle \mathrm{ARE}}$ mice develop chronic CD-like ileitis and arthritis. The sustained overexpression of TNF results in an enhanced Th1/Th17 response. Not only TNF production by immune cells, but also by IECs and myofibroblasts contributes to the development of ileitis in this model (Bamias et al, 2013; Jones-Hall \& Grisham, 2014). Moreover a role for the microbiota has also been elicited, since iron depletion in these mice resulted in reduced inflammation due to a shift in microbiota composition (Werner et al, 2011).

\section{Immune-manipulated models}

The T cell transfer model was developed by Powrie et al. in 1993 and is one of the key IBD mouse models that changed the research field in the last 20 years (Powrie et al, 1993). CD4+ T cells in the mouse can be subdivided into two fractions based on the level of expression of the CD45RB determinant. CD45RB high $C D 4+T$ cells produce high levels of IL-2 and contain naïve T cells but lack Tregs. CD45RB ${ }^{\text {low }}$ CD4+ T cells produce both IL-2 and IL-4, and contain both naïve T cells and Tregs.

Adoptive transfer of $\mathrm{CD} 45 \mathrm{RB}^{\text {high }} \mathrm{CD} 4+\mathrm{T}$ cell population obtained from lymph nodes or spleen from healthy WT mice into severe combined immunodeficient (SCID) or Rag-1/-2 knock-out mice resulted in a lethal wasting disease with severe mononuclear cell infiltrates into the colon and elevated levels of IFN- $\gamma$ mRNA. In contrast, adoptive transfer of the CD45RB ${ }^{\text {low }}$ subset or unfractionated CD4+ T cells did not result in wasting disease or colitis. Interestingly, the co-

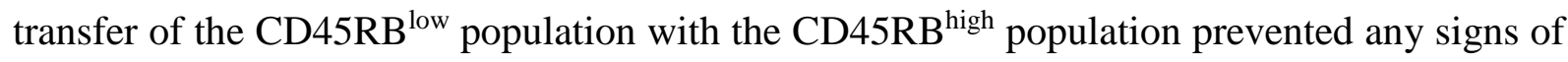
disease. This model has demonstrated the critical role of Tregs as important immunomodulatory cells. The mice develop clinical signs in both colon and small intestine and these are closely related to those in CD patients. Moreover, a mixed Th1/Th17 inflammatory response is elicited and a role for the microbiota has been suggested. A comparative study regarding colonic gene expression profiles in different IBD mouse models has pointed out that the T cell transfer model 
has a gene expression profile that is the most similar to that observed in human disease. Finally, the model can also be used to investigate the efficacy of therapeutic agents in vivo.

\section{Spontaneous models}

The Senescence Accelerated Mouse Prone 1 (SAMP1)/Yit model was first described by Matsumoto and colleagues who obtained this substrain by selective breeding of AKR mice used in senescence studies (Matsumoto et al, 1998). In these aging studies, one strain SAMP1, developed skin lesions and was backcrossed over 20 times to eventually obtain the SAMP1/Yit mice. This substrain did no longer develop the accelerated senescence phenotype, but instead presented with spontaneous ileitis under SPF conditions. SAMP1/YitFc mice represent another substrain which was developed by Cominelli and colleagues, and these mice also exhibited gut inflammation with striking similarities to CD (Rivera-Nieves et al, 2003). In both SAMP1/Yit and SAMP1/YitFc mice, inflammation develops in all mice by 30 weeks of age and is restricted to the terminal ileum and to a lesser extent the cecum. Mucosal fibrosis was also observed and some SAMP1/YitFc developed strictures and perianal fistulas (Rivera-Nieves et al, 2003) making this model an ideal tool to study similar complications in CD patients. A mixed Th1/Th2 cytokine profile was observed and the severity of the disease phenotype has been shown to be dependent on the microbiota (Jones-Hall \& Grisham, 2014).

\section{Chemically-induced models}

Dextran sodium sulphate (DSS) is a sulphated polysaccharide that can induce colitis via oral administration in the drinking water of mice. Depending on the concentration, duration and frequency of the administration, the mice develop acute or chronic colitis or even colitisinduced dysplasia. The molecular weight (MW) of DSS is highly variable and ranges from 5 $\mathrm{kDa}$ to $1400 \mathrm{kDa}$. Several studies have shown that $40 \mathrm{kDa}$ DSS induces the most reproducible results with severe colitis in the middle and distal part of the colon, whereas lower or higher MW of DSS are not sufficient. Acute colitis is induced by continuous administration of 2-5\% 
DSS for 4-9 days, whereas chronic colitis is induced by cyclic administrations at lowered concentrations of DSS (Breynaert et al, 2013; Perse \& Cerar, 2012). The mice typically develop clinical manifestations of weight loss, bloody diarrhea and anemia. Histological changes in the acute model include mucin depletion, epithelial degeneration, erosions and neutrophil infiltration in the lamina propria and the submucosa. Cryptitis and crypt abscesses are frequently seen in human disease, but are rarely reported in DSS-induced colitis. Chronic DSS-induced colitis is characterized by mononuclear cell infiltration, crypt distortion and fibrosis with thickening of the mucosa and muscularis propria (Breynaert et al, 2013).

DSS has a direct toxic effect on colonic epithelial cells with disruption of the epithelial barrier integrity and influx of luminal antigens and microorganisms as a result. This leads to a sustained inflammatory response with increased expression of several inflammatory mediators. In the acute model a mainly Th1/Th17 cytokine profile is seen, whereas in the chronic model this evolves to a more Th2 mediated cytokine profile. Recently, it has been shown that the gene expression profile is different in the acute and chronic model of colitis (Breynaert et al, 2013). The acute model seems to be mainly mediated by an innate immune response since DSS colitis can also be induced in SCID and Rag-1/-2 deficient mice which lack T and B cells (Axelsson et al, 1996). However, more recent data suggest a role for the adaptive immune system since pro-inflammatory $\mathrm{T}$ cells were shown to be elicited towards specific oral antigens in DSS induced colitis (Morgan et al, 2013). Also in this model, the gut microbiota seem to play an important role. Some studies have shown that DSS colitis does not develop in gnotobiotic mice, but rapidly emerges after reconstitution with commensal bacteria (Nell et al, 2010). Other studies point to a more specific role of the microbiota in the pathogenesis of DSS colitis. In a study by Okayasu et al., it was shown that increased numbers of Enterobacteriaceae, Bacteriodaceae and Clostridium spp. were present in the colon of mice affected with DSS colitis (Okayasu et al, 1990). Furthermore, treatment of DSS-colitis mice with antibiotics 
reduced the infiltration of neutrophils in the mucosa and restored histological features (Hans et al, 2000). However, not all authors agree on the pathological role of the microbiota in DSSinduced colitis. This is mainly due to genetic background as well as environmental differences (vide infra).

The advantages of the DSS model are its simplicity, relatively low cost, rapid onset of inflammation, reproducibility and histopathological similarity to UC. It is clear that this model is primarily an injury-induced inflammatory model with a direct disruption of the epithelial barrier. Therefore it serves as an excellent model to study acute inflammation and barrier disruption as well as the secondary effect of the microbiota contributing to the susceptibility and severity of disease. Moreover, recent advances in the characterization of the chronic DSS model have indicated a role for the adaptive immune system in chronic inflammation and fibrosis, making the DSS model an attractive all-round IBD mouse model.

Administration of the hapten trinitrobenzene sulfonic acid (TNBS) intrarectally together with ethanol $(\mathrm{EtOH})$ induces an erosive, self-limiting colitis. Simultaneous delivery of EtOH is necessary to cause a disruption of the mucosal barrier allowing further translocation of the water soluble TNBS into to submucosa. Subsequently, TNBS causes a delayed-type hypersensitivity (DTH) reaction by haptenizing colonic autologous or bacterial proteins. Clinical manifestations include diarrhea, rectal bleeding and prolapse, and weight loss. It affects the entire colon and transmural inflammation is observed with histological features including ulceration, edema and loss of goblet cells. After two months, the disease further develops into chronic colitis with lymphoid aggregations and fibrosis. The model is mainly driven by a Th1/Th17 cytokine profile and resembles human CD. The advantages of the TNBS model are its simplicity and low cost. However, the model is highly variable and difficult to reproduce due to differences in methodology and genetic background. 


\section{Importance of the genetic background}

Many of the above described animal models are not readily reproducible due to the use of different mouse strains with a different genetic background. For example, the $\mathrm{C} 3 \mathrm{H} / \mathrm{HeJ}$ substrain and 129SvEv strain are highly susceptible to develop colitis after deletion of IL-10, in contrast with the C57BL/6J and Balb/c strains (Mahler \& Leiter, 2002). Moreover, SJL/J and Balb/c mice are susceptible to TNBS-induced colitis, whereas C57BL/6 and C57BL/10 are relatively resistant (Scheiffele \& Fuss, 2002). The influence of the genetic background is most elaborately described for the DSS model. C3H/HeJ(Bir), NOD/LtJ and NOD-scid mice are highly susceptible to DSS-induced colitis, whereas C57BL/6, 129SvPas and DBA/2J mice are more resistant to development of DSS lesions (Mahler et al, 1998; Perse \& Cerar, 2012). Balb/c mice appear to be more resistant to DSS than C57BL/6, because after DSS cessation, Balb/c mice recovered whereas C57BL/6 mice developed chronic disease (Melgar et al, 2005). Moreover, male mice appear to be more susceptible to DSS since more severe lesions in the colon were observed. The genetic factors underlying the difference in DSS susceptibility in various mice strains remain unknown. In conclusion, the importance of the genetic background reaches far beyond the use of IBD mouse models and has an impact on all animal and human research as it strongly influences the obtained phenotype or response to treatment.

\subsection{Tissue remodeling}

The disease course of an IBD patient is heterogeneous and very difficult to predict. Several stages are involved in the onset and perpetuation of IBD. Both environmental and genetic factors lie at the basis of the onset of disease and can lead to an impaired barrier function. The leaky barrier thus allows translocation of microbial products which initiates an acute inflammatory response with activation of innate and adaptive immune cells. Failure of regulatory mechanisms and continuous production of pro-inflammatory mediators eventually leads to a state of chronic inflammation. Tissue damage and subsequent tissue repair are 
hallmarks of chronic inflammation. An imbalance in tissue remodeling mechanisms can cause several serious complications in IBD patients e.g. fibrosis and fistulas. Multiple factors are involved in tissue remodeling, including the site and duration of inflammation, soluble factors and the type of mesenchymal cell response (Rieder \& Fiocchi, 2013). Inflammation has multiple physiological purposes under controlled conditions. However, when inflammation is excessive, several pathological processes occur e.g. cell damage or death, disruption of architecture and function, fibrosis, autoimmunity, metaplasia or cancer.

Intestinal fibrosis is a universal feature of both $\mathrm{UC}$ and $\mathrm{CD}$, and is defined as excessive extracellular matrix (ECM) formation with proliferation and activation of myofibroblasts. In $\mathrm{UC}$, fibrosis manifests as thickening of the colonic mucosa and submucosa, whereas in $\mathrm{CD}$, the entire small or large bowel wall is affected including the muscularis propria and serosa. The thickening of the bowel wall can lead to narrowing of the lumen and eventually formation of strictures and bowel stenosis. Aberrant tissue remodeling and intestinal fibrosis have a major clinical impact in IBD. More than $30 \%$ of CD patients evolve towards a fibrostenosing phenotype with progressive narrowing of the lumen. As a consequence, up to $80 \%$ of CD patients need to undergo surgery at least once during their disease course and even after surgery in $>45 \%$ of the patients these obstructions are recurrent (Rieder et al, 2007). In contrast to recent advances in anti-inflammatory treatment, little therapeutic progress has been made regarding anti-fibrotic treatment. The main reason for this is that the early triggers leading to intestinal fibrosis in response to inflammation are unknown. They are thought to be at least partially distinct from inflammatory pathways since current available treatments have not been able to diminish the incidence of fibrosis. In addition, only a few animal models of fibrosis exist and there is a lack of reliable biomarkers (Rieder et al, 2014; Rieder et al, 2012). 


\section{INTERACTIONS}

METALLOPROTEINASES

\section{METALLOPROTEINASES}

AND BALANCES BETWEEN MATRIX

AND TISSUE INHIBITORS OF

\subsection{Matrix metalloproteinases (MMPs)}

Chronic inflammation and aberrant tissue remodeling with excessive accumulation or degradation of extracellular matrix ECM components are hallmarks in IBD. Matrix metalloproteinases (MMPs) are able to cleave ECM components and are thought to be predominant proteases involved in the pathogenesis of IBD. The human MMP family exists of 23 zinc-dependent endopeptidases encoded in 24 genes including duplicated MMP-23 genes. They can be subdivided into several groups based on their domain structure and substrate specificity: collagenases (MMP-1, -8, -13), gelatinases (MMP-2, -9), stromelysins (MMP-3, 10, -11), matrilysins (MMP-7, -26), membrane-type MMPs (MT-MMP-1 to -6) and others (Ravi et al, 2007; Vandooren et al, 2013b; Visse \& Nagase, 2003). The primary structures of MMPs differ, but they all share a conserved catalytic domain with a zinc ion in the active site, a signal peptide and a propeptide. In addition, most MMPs - except for matrilysins and MMP23 - have a haemopexin-like domain which plays a role in substrate recognition, induction of auto-activation and binding to inhibitors and cell surface receptors. Moreover, gelatinases have three fibronectin repeats located in between the active site and the metal binding site which are important for the binding and catalysis of large substrates such as elastins and denatured collagens or gelatins. Uniquely, gelatinase B (or MMP-9) contains an O-glycosylated domain inserted between the zinc-binding and the haemopexin-like domain which facilitates enzyme flexibility. MT-MMPs have a membrane anchor consisting of a type-I transmembrane domain with a cytoplasmic domain or a glycosyl-phosphatidyl-inositol (GPI) anchor (Vandooren et al, 2013b; Visse \& Nagase, 2003). In figure 2, panel A the MMP domain structure is illustrated based on MMP-9 as a model molecule. 
The presence of MMPs is generally low in control or uninflamed tissues. Indeed, the expression, secretion, activation and activity of MMPs are tightly controlled. For example, the expression of MMPs is transcriptionally controlled by many factors including cytokines, growth factors, hormones, cell-cell and cell-matrix interactions and these processes have been reviewed previously by several groups (Chakraborti et al, 2003; Yan \& Boyd, 2007). Furthermore, MMPs can also be controlled post-transcriptionally and through epigenetics. For example, oxLDL regulates MMP-2 and MMP-9 gene expression by inducing miR-29 which downregulates DNA methyltransferase $3 b$ (Chen et al, 2012). Upon secretion, most MMPs are inherently inactive since they are secreted as latent pro-enzymes and require proteolytic activation by cleavage of the propeptide, a process called 'the cysteine switch' (Van Wart \& Birkedal-Hansen, 1990). Briefly, a cysteine containing sequence (PRCGXPD) in the propeptide is able to interact with the catalytic zinc ion and thus keeps the enzyme inactive. This interaction can be interrupted either by removal of the propeptide or by covalent modification of the propeptide cysteine residue. In the active protease, the interaction with the propeptide cysteine residue is replaced by a water molecule. Other interaction sites of the zinc ion are three histidine residues present in the zinc binding motif HEXXHXXGXXH of the catalytic domain. Finally, the activity of MMPs is regulated by tissue specific location of the enzyme and inactivation by endogenous inhibitors. The latter include tissue inhibitors of MMPs which are one of the subjects of this review.

\subsection{Tissue inhibitors of metalloproteinases (TIMPs)}

Tissue inhibitors of metalloproteinases (TIMPs) are generally known for their inhibitory action on MMP-mediated catalysis. The mammalian TIMP family has four members (TIMP-1 to TIMP-4) which share sequence homology and structural identity (between 41 and 52\%) at the protein level (Baker et al, 2002; Brew et al, 2000; Edwards et al, 1996; Visse \& Nagase, 2003; Woessner, 2002). The 4 TIMPs have different expression patterns. TIMP-2 expression is 
constitutive and found throughout the body, whereas TIMP-1, -3 and -4 expression is inducible and often tissue-specific. TIMP-1 is mainly expressed in reproductive organ systems, whereas TIMP-3 is expressed in heart, thymus and kidney and TIMP-4 is expressed in brain, heart, skeletal muscle and ovary (Chirco et al, 2006).

Human TIMPs are 184-194 amino acid long molecules that contain 6 disulphide bridges. The 23 human MMPs are composed, in different degrees, of various domains and interact with 4 human TIMPs in a 1:1 stoichiometry (Figure 2, panel A). Most TIMP-MMP-interactions have an inhibitory constant $(\mathrm{Ki})$ of around $1 \mathrm{nM}$, with the exception of a rather weak interaction between MT1-MMP and TIMP-1 (Ki 100 nM) and the extremely strong interaction between MMP-2 and TIMP-2 (Ki 1 fM). Whereas TIMP-1 mainly inhibits MMP-9, TIMP-2 is the major inhibitor of MMP-2 and -3 (Gomis-Ruth et al, 1997; Nagase et al, 2006). TIMPs are comprised of an N-terminal domain of $\sim 125$ amino acids and a C-terminal region of $\sim 65$ amino acids. The $\mathrm{N}$-terminal domain interacts with the zinc-binding domain of MMPs and is sufficient for MMP inhibition (Figure 2, panel A). The C-terminal domain is important for protein-protein interactions and can exert MMP-independent functions by binding to cell surface receptors (Butler et al, 1999; Morgunova et al, 2002; Ogata et al, 1995). Although the mechanism of TIMP inhibition of catalytic activity has been elucidated based on the crystal structures of MMP-TIMP complexes, the exact interaction modes of TIMPs with MMPs remain unclear. For example, one might wonder whether the interaction of TIMP-1 with the highly flexible MMP9 monomer molecule might involve simultaneous binding of C-terminal TIMP-1 to the haemopexin-like domain and N-terminal TIMP-1 to the catalytic site (Figure 2, panel A, right molecular interaction). If so, the formation of MMP-9 complexes, for example the NGALMMP-9 complex, might influence TIMP-1 inhibition of MMP-9 proteolysis (Figure 2, panel

\section{A, left molecular interaction).}


Comparable to additional signaling functions of MMPs (Bailon et al, 2014; Chirco et al, 2006; Lambert et al, 2004; Moore \& Crocker, 2012; Stetler-Stevenson, 2008), TIMPs also exert multiple MMP-independent functions. TIMP-1 and TIMP-2 were shown to exert growthpromoting effects in a wide variety of cell types including normal cells, cancer cells and lymphoid cells. As an example, TIMP-1 was discovered to have erythroid potentiating activity (EPA), in other words it acts as a hematopoietic cytokine for red blood cells (Docherty et al, 1985). Surprisingly, both TIMPs also exerted growth-inhibiting effects in certain cell types including endothelial cells and several carcinoma cells (Lambert et al, 2004). TIMP-3 was shown to stimulate the proliferation of growth-retarded, non-transformed cells under low serum condition, whereas TIMP-4 inhibited growth of G401 Wilms tumor cells. In addition to their cell growth properties, TIMPs have also been shown to exert anti-apoptotic activity. In several cell types, TIMP-1 was associated with a reduced susceptibility for apoptosis. In contrast, the effect of TIMP-2 or TIMP-3 on programmed cell death is not clear due to conflicting data in the literature. However, TIMP-4 seems to have similar effects and can inhibit apoptosis and therefore promote tumor growth. Importantly, these growth promoting/inhibiting or antiapoptotic activities of TIMPs can be both MMP-dependent and MMP-independent (vide infra). In recent studies it was shown that TIMPs are also involved in cell signaling by binding to cell surface receptors. To date, two TIMP-1 receptors have been identified: a CD63- $\beta 1$ integrin complex in a breast cancer cell line (MCF10A) (Jung et al, 2006) and $\alpha v \beta 3$ integrin in an osteosarcoma cell line (MG-63) (Tsagaraki et al, 2010). Strong evidence exists that TIMP-1 exerts its cell growth and anti-apoptotic activities through interaction with these receptors (Moore \& Crocker, 2012). Recently, Kobuch et al. demonstrated that TIMP-1 signaling via CD63 triggers granulopoiesis and neutrophilia in mice (Kobuch et al, 2015). Furthermore, TIMP-2 has been shown to bind to $\alpha 3 \beta 1$ integrin on endothelial cells and thereby inhibits endothelial cell proliferation and angiogenesis (Seo et al, 2003). An anti-angiogenic effect has 
also been described for TIMP-3 by binding to vascular endothelial growth factor receptor 2 (VEGF-R2). TIMP-3 hereby competes with VEGF-A for binding to the receptor and thus acts as a VEGF-A antagonist (Qi et al, 2003). In addition, TIMP-3 may also interact with angiotensin II type 2 receptor to inhibit angiogenesis (Kang et al, 2008). Most of these cell-signaling functions have been shown to be MMP-independent.

Finally, TIMPs are also implicated in MMP activation. For example, one of the mechanisms of proMMP-2 activation includes the formation of a complex comprising proMMP-2, active MT1MMP/MMP-14 and TIMP-2. Once this complex is formed at the cell surface, MT1-MMP cleaves proMMP-2 which results in active MMP-2 (Figure 2, panel B) (Strongin et al, 1995; Ward et al, 1994). In the absence of TIMP-2, MT1-MMP can be subject to autocatalysis resulting in a release of the entire catalytic domain and the generation of a membrane-bound inactive form of MT1-MMP (Hernandez-Barrantes et al, 2000). TIMPs can therefore also regulate the activation status of different MMPs.

Both MMPs and TIMPs have a wide range of activities. Therefore these molecules need to be carefully controlled during physiological conditions and tissue remodeling. Imbalances of MMPs/TIMPs can result in pathological processes. Whereas excess activity of MMPs in IBD can contribute to destruction of the intestinal barrier and granulation, overproduction of TIMPs might restore the intestinal barrier and potentially lead to fibrosis (Figure 2, panel C).

\section{MMP SUBSTRATES IN IBD}

At first, MMPs were considered as general proteases of extracellular matrix components such as gelatins, collagens and elastins (Van den Steen et al, 2002; Vandooren et al, 2013b). Now it is clear that this view was too simplistic. In the last years, it was discovered that MMPs can cleave a wide range of molecules such as soluble factors, membrane receptors, adhesion factors, signaling molecules, cytoskeleton proteins and even molecules inside the nucleus (Cauwe \& Opdenakker, 2010; Cauwe et al, 2007; Rodriguez et al, 2010). The truncation or degradation of 
molecules by MMPs imposes an alteration in the activities of these proteins and consequently triggers physiological changes in cells and their environment. Furthermore, also non-catalytic functions have been described whereby MMPs act as cell ligands, activating signaling pathways (Redondo-Munoz et al, 2010), or even act as intracellular transcription factors (Eguchi et al, 2008; Marchant et al, 2014).

Many MMP substrates are important molecules within mucosal and submucosal layers and might contribute to the development and progression of IBD. In Figure 3, some processes that might be mediated by MMPs in the intestinal epithelial layer and immune cells are illustrated. For example, besides allowing cell migration, degradation of collagens can result in the generation of collagen fragments with inherent functionalities. MMP-3, -7, -9, -13 and -20 are able to generate biologically active endostatin from the C-terminal non-collagenous NC1 domain of type XVIII collagen (Heljasvaara et al, 2005). Endostatin inhibits endothelial cell proliferation, migration, angiogenesis and tumor growth (Heljasvaara et al, 2005). Prolineglycine-proline (PGP) is also a compound derived from collagen and is generated by the combined action of MMP-8, MMP-9 and prolyl endopeptidase (PE). PGP has chemotactic effects on neutrophils and acts via the chemokine receptor CXCR2. Moreover, Koelink et al. demonstrated that PGP levels were increased in intestinal tissue of patients with IBD and in the intestine of DSS-treated mice. Whereas MMP-8 and MMP-9 levels were elevated in the intestine of patients with IBD, PE was present at equal levels as observed in control tissues. Furthermore, PGP neutralization in the DSS-induced colitis mouse model led to a significant reduction in neutrophil infiltration in the intestine (Koelink et al, 2014). Besides collagen, the ECM can also contain matrix-bound VEGF. VEGF is the main mediator of blood vessel formation during development and in pathological conditions and MMPs are able to induce VEGF bioavailability through intramolecular processing. Specifically, some MMPs can cleave matrix-bound isoforms of VEGF, releasing soluble fragments. In tumors, MMP-cleaved VEGF 
promotes capillary dilatation of existent vessels and mediates neovascularization. In contrast, MMP-resistant VEGF supports extensive growth of thin vessels with multiple and frequent branch points. Matrix-bound VEGF and non-tethered VEGF therefore provide different signaling outcomes. These findings reveal a novel aspect in the regulation of extracellular VEGF that holds significance for vascular patterning and could be important in IBD (Lee et al, 2005b). MMPs are also mediators of inflammation by truncating chemokines into more or less potent molecules (Opdenakker et al, 2001a). The prototypic example of this is IL-8/CXCL8, which is potentiated at least 10-fold by active MMP-9/gelatinase B (Van den Steen et al, 2000). In addition, MMP-13 is able to cleave membrane-bound TNF into soluble TNF. Indeed, in a DSS-induced colitis model it was shown that MMP-13-mediated shedding of TNF contributes to IBD (Vandenbroucke et al, 2013). IL-1 $\beta$, a cytokine whose overexpression has been associated with IBD (Shi et al, 2007), can be degraded by MMP-1, -3 and -9 which diminishes its pro-inflammatory, metastatic and angiogenic effects (Ito et al, 1996). In a DSS mouse model for acute colonic injury in MMP-7 KO mice it was found that MMP-7 contributes to reepithelialization and controls the transepithelial influx of neutrophils (Swee et al, 2008). Moreover, MMP-7 KO mice produce procryptdins but not mature cryptdins ( $\alpha$-defensins) and have a general upregulation of IL-1 $\beta$ in the intestine (Shi et al, 2007). Cryptdins or $\alpha$-defensins are antibiotic peptides which are secreted by epithelial cells and granulocytes. Interestingly, MMP-7 can regulate $\alpha$-defensins by processing precursors into mature $\alpha$-defensins (Shirafuji et al, 2003; Wilson et al, 1999). Besides killing bacteria by membrane disruption, $\alpha$-defensins can act as pro-inflammatory molecules by inducing the secretion of IL- 6 by macrophages thereby contributing to intestinal leakage and inflammation (Vandenbroucke et al, 2014).

MMPs can also cleave several proteins implicated in maintenance of an intact epithelial barrier (Figure 3). For example, MMP-3, -7 and MT1-MMP are able to cleave cadherins, whereas occludins can be degraded by MMP-2, $-3,-7$ and -9 . Furthermore, claudin-5 is a substrate of 
MMP-3 (Cauwe et al, 2007). Integrins are both receptors and mediators of cell-interactions (cell-cell and cell-ECM adhesion molecules). In IBD and many other diseases these adhesion molecules have been associated with leukocyte trafficking. Therefore, anti-adhesion molecule strategies have already been studied for the development of new therapeutics in the management of IBD (vide supra). MMPs are also mediators of integrins. Maturation of pro-integrins can be aided by MT1-MMP (Deryugina et al, 2000) and, once activated; integrins can function as a docking complex for MMP-9 (Redondo-Munoz et al, 2008). Finally, MMP-7 is also able to inactivate pro- $\alpha 4$-integrin (Powell et al, 1999). MMPs (MMP-3 and MMP-7) can either induce or reduce apoptosis by releasing soluble Fas ligand (sFasL) (Tanaka et al, 1998). FasL is a homotrimeric type II membrane protein, mostly found on T lymphocytes, macrophages and NK cells. In the colonic mucosa FasL-containing cells comprise mainly lymphocytes in the lamina propria, in areas adjacent to the epithelium. Recent studies show that systemic and local Fasmediated apoptosis is promoted in UC and might be involved in UC pathogenesis (Souza et al, 2005; Yukawa et al, 2002). Finally, the cell surface associated mucin MUC1, which acts as an antibacterial intestinal barrier molecule (Linden et al, 2009), can also be cleaved by MT1-MMP (Thathiah \& Carson, 2004).

Besides extracellular and cell-bound proteins, MMPs cleave many cytoskeletal proteins, including proteins implicated in protein biosynthesis, redox regulation, signal transduction, apoptosis, protein folding and even the regulation of translation and transcription (Cauwe et al, 2009). Also cytoskeletal proteins can be degraded by MMPs for example actins, filamins and tubulins. The processing of these structural molecules can induce changes in morphology and migration of endothelial and immune cells. The degradation of both extracellular and intracellular structural molecules can contribute to impairment of the intestinal epithelial barrier in IBD. 


\section{ASSOCIATION STUDIES OF MMPS IN IBD}

\subsection{MMP genetic association studies}

Strong evidences exist for a genetic component in IBD since a higher disease concordance is found in monozygotic versus dizygotic twins (Tysk et al, 1988), a higher frequency of IBD occurs in families and ethnic groups (Kurata et al, 1992; Russel et al, 1997) and 201 susceptibility loci have been described in IBD patients (Cleynen et al, 2016; Cleynen et al, 2013; Ellinghaus et al, 2016; Franke et al, 2010; Jostins et al, 2012; Liu et al, 2015; Van Limbergen et al, 2014). Interestingly, one of these 201 IBD susceptibility loci has been identified for MMP-9 (rs1569723) (Jostins et al, 2012). Moreover, several functional single nucleotide polymorphisms (SNPs) in MMP genes have been described (as reviewed by Langers et al. (Langers et al, 2011)).

Multiple studies were conducted to investigate whether the previously identified SNPs in MMP genes were associated with IBD (Table 2). First, Satsangi et al. investigated the contribution of the MMP-3 6A/5A genotype to the pathogenesis of primary sclerosing cholangitis (PSC) (Satsangi et al, 2001). PSC is a cholestatic liver disease that shares pathologies with IBD and $50-80 \%$ of the patients also suffer from IBD (Karlsen \& Boberg, 2013). In this study, $82 \%$ of the PSC patients presented with concomitant UC. Strong evidence was found that the MMP-3 6A/5A genotype may influence susceptibility and disease progression in PSC, as well as the development of portal hypertension (Satsangi et al, 2001). Moreover, the MMP-3 5A allelic frequency, carriage rate and homozygosity rates were increased in PSC compared with UC, although no differences were found between UC and healthy subjects. In addition, an association of MMP-2 -1306 C/T was found with disease severity in PSC, although concomitance with IBD did not change the association strength (Korkmaz et al, 2014). The MMP-9 -1562 C/T genotype was not found to be associated with disease severity in PSC (Korkmaz et al, 2014). A highly significant association was found between overtransmission of 
the 5A allele in MMP-3 and CD (Pender et al, 2004). In contrast, no association was found between UC and the MMP-3 5A/6A genotype. The association of the MMP-3 5A allele with CD was apparent only in individuals carrying one or more mutations in the CARD15 gene and was linked with various CD complications, including stenosis, fistulizing disease, previous surgical resection and ileal or right colonic disease.

No significant differences in genotype distribution at -1607 1G/2G (MMP-1) and -1306 C/T (MMP-2) were found between IBD patients and controls (Meijer et al, 2007c). Moreover, allele frequencies of SNPs with G in the MMP-1 gene and C in the wild-type MMP-2 gene were not different between both groups. MMP-3 and MMP-9 genotype distribution at -1613 5T/6T and $-1562 \mathrm{C} / \mathrm{T}$, respectively, did also not differ between IBD patients and controls. When investigating the effect of the SNPs on CD phenotype, it was found that the 5T5T MMP-3 genotype was associated with a lower chance of colonic involvement at first endoscopic or radiologic evaluation. However, the 5T5T genotype was also associated with a major risk for development of stenotic complications. The lack of genetic associations of MMP-1, -2 , and -9 was also described in earlier linkage studies (Ma et al, 1999; Rioux et al, 2000; Satsangi et al, 1996). Explants with at least one $2 \mathrm{G}$ allele at MMP-1 -1607 or 5T allele at MMP-3 -1613 were shown to express more protein in medium compared to explants with alternative allelic composition, although these results were not statistically significant (Meijer et al, 2007b). Moreover, infliximab significantly decreased protein expression in explants with CC genotype at MMP-9 -1562, with at least one 2G allele at MMP-1 -1607 and with a 5T allele at MMP-3 1613. At mRNA level, explants with CC genotype at MMP-2 -1306 had higher MMP-2 expression compared to other genotypes (not statistically significant) and only explants with 6T6T genotype at MMP-3 -1613 had significantly decreased MMP-3 expression.

In a Caucasian New Zealand cohort of 419 UC patients SNPs in MMP-1, -2, -3, -7, -8, -9, -10, $-12,-13$ and -14 were further investigated (Morgan et al, 2011). Significant associations with 
several disease parameters were found for SNPs in MMP-3, -8, -10 and -14. Five SNPs in MMP-10 were found to be associated with UC and were further analyzed for phenotypic associations. In the MMP-10 gene, rs4431992 was associated with extra-intestinal manifestations, whereas rs12290253 was associated with the female gender, age at first diagnosis above 40 years, pancolitis, bowel resection and being a smoker at diagnosis. Three SNPs in MMP-8 were significantly associated with UC and all three SNPs were associated with UC in females and with patients who were diagnosed after 40 years of age. Moreover, rs3765620 was associated with disease of the left colon and rs1267284 with patients diagnosed between 17 and 40 years pancolitis, bowel resection, extra-intestinal manifestations and smoking at diagnosis. One SNP in MMP-3 was associated with UC and found to be associated with females, age at first diagnosis $17-40$ years, left colonic disease, smoking at diagnosis and having family with IBD. These associations could not be confirmed in a Dutch cohort of 500 UC patients. However, data from an international meta-analysis provides some support for these findings (Anderson et al, 2011). Finally, SNPs in MMP-1 (-1607), MMP-7 (-153 and -181) and MMP-9 (exon 6) were investigated in 75 patients with collagenous colitis and 334 healthy controls. The carrier frequency of the allele GG of the coding SNP MMP-9 in exon 6 was found to be significantly higher in patients with collagenous colitis compared with healthy controls and this was reflected by an odds ratio of 1.9 for increased risk of collagenous colitis (Madisch et al, 2011). None of the other SNPs of MMP-1 or -7 were found to be associated with collagenous colitis.

As a conclusion, SNP association studies indicate some associations of the stromelysins MMP3 and MMP-10 with increased risk for IBD, whereas SNPs in the neutrophil collagenase MMP8 gene are associated with lowered risk for IBD. Many MMP SNPs are clearly not associated with IBD, weakening the possibility that these MMPs may become therapeutic targets. 


\subsection{MMP messenger RNA (mRNA) expression studies}

Since MMPs play an important role in tissue remodeling, aberrant expression has been implicated in several diseases, including IBD. Transcription of MMPs is controlled by diverse signaling pathways (Van den Steen et al, 2002) and for IBD these have recently been described in detail by O'Sullivan and colleagues (O'Sullivan et al, 2015). An overview of studies in which mRNA expression of MMPs in IBD patients was investigated is shown in Table $\mathbf{3}$ and the cellular MMP mRNA expression patterns are symbolized in Figure 4. Whereas 2-fold increases are assumed to be significant, in many cases alterations at mRNA levels are substantially higher. To give the reader better information about the degrees of fold-changes, we detail here exact numbers for critical comparisons.

\section{In vitro/ex vivo mRNA expression studies}

In control human fetal small intestine explants, $M M P-1$ and -2 mRNAs were detected, whereas MMP-3 and -9 mRNAs were low or absent. In T cell-stimulated explants, however, the expression of $M M P-1,-2,-3$ and -9 was found to be increased compared to control explants (Pender et al, 1997). In an ex vivo model of T cell-mediated gut injury, a marked upregulation of MMP-10 and -12 mRNA expression was observed and this was correlated with mucosal destruction (Salmela et al, 2002). Moreover, MMP-1, -3 and -9 mRNA expression was upregulated following T cell activation, but no change in $M M P-2,-7,-8,-9,-11,-13,-14-17$, or -19 mRNA expression was observed.

The influence of infliximab on the expression of $M M P-1,-2,-3$ and -9 in intestinal mucosal explants was evaluated by Meijer and colleagues (Meijer et al, 2007b). Addition of infliximab to the explant cultures led to a decrease in basal MMP mRNA expression, particularly of $M M P$ 1 and -3 , in CD patients and controls, but not in UC patients. The influence of infliximab on the expression of $M M P-2$ and -9 was further investigated in whole blood cultures after stimulation with lipopolysaccharide (LPS) (Gao et al, 2007). MMP-2 mRNA levels were not detectable, 
whereas MMP-9 mRNA levels were increased 16-fold in leukocytes of both CD patients and healthy subjects after 24 hours stimulation with LPS. Addition of infliximab to the cultures resulted in $80 \%$ reduction of the increased $M M P-9$ mRNA expression levels. Furthermore, Gordon and colleagues investigated $M M P$ expression in gut plasma cells isolated from mucosal tissue of IBD patients (Gordon et al, 2008). The plasma cells from IBD patients expressed significantly higher levels of $M M P-3$ transcripts compared with plasma cells from controls. The same authors further investigated whether immunomodulatory drugs influenced $M M P$ expression in cultured gut lamina propria mononuclear cells (LPMCs). They found that the phosphodiesterase 4 (PDE4) inhibitor CC-10004, but not thalidomide or lenalidomide, inhibited MMP-3 mRNA production in IBD patients (Gordon et al, 2009). These results are in line with the failure of lenalidomide in a recent randomized controlled trial in $\mathrm{CD}$ as well as for the evaluation of CC-10004 as a novel oral therapy in the treatment of IBD.

The expression of $M M P-1,-2,-3,-9$ and $M T 1-M M P$ mRNAs was also investigated in human fetal mesenchymal cell lines. A high expression of MT1-MMP and MMP-3 mRNA was found in activated fibroblast-like cells at the ulcer edge where vascular cell adhesion molecule-1 (VCAM-1)-positive cells were present (Pender et al, 2000). In intestinal myofibroblast cultures, no differences were observed in $M M P-1,-2$ and -3 expression between control, UC and CD patients, whereas MMP-9 mRNA expression was below the detection limit (McKaig et al, 2003). Furthermore, the effect of $\mathrm{T}$ cell-derived IL-21 on $M M P$ expression in intestinal fibroblasts was investigated (Monteleone et al, 2006). MMP-1, -2, -3 and -9 mRNA transcripts were detected in untreated intestinal fibroblasts. However, stimulation of the cells with IL-21 did not enhance $M M P$ mRNA expression. By contrast, TNF- $\alpha$ was able to enhance $M M P-1$, $M M P-3$ and $M M P-9$ mRNA expression, but not $M M P-2$ mRNA.

Stimulation of Caco- 2 cells with IL- $1 \beta$ or TNF- $\alpha$ led to a dose-dependent increase in expression of MMP-9 mRNA (Gan et al, 2001). In contrast, LPS failed to induce expression of $M M P-9$, 
suggesting that an inflammatory reaction leading to cytokine release is a necessary step for the induction of MMP-9 release in intestinal epithelial cells. Treatment of Caco-2 cells with rosiglitazone, a peroxisome proliferator-activated receptor- $\gamma$ (PPAR- $\gamma$ ) agonist, was able to significantly reduce the secretion of $M M P-9$, indicating that agents that activate PPAR- $\gamma$ may have therapeutic use in patients with IBD. The expression of $M M P-1,-2,-3,-7,-8,-9,-10,-11$, $-12,-13$ and -14 mRNA was also investigated in human colonic epithelial cells (CEC) (Pedersen et al, 2009). HT-29 and DLD-1 cells expressed several MMPs in control conditions and the expression of $M M P-3,-10$ and -13 mRNAs increased significantly after TNF stimulation. Moreover, MMP-1, -3, -7, -9, -10 and -12 transcripts were detected in CECs and were found to be significantly increased in cells from inflamed IBD mucosa, except for MMP-12.MMP-2 and -8 mRNAs were expressed inconsistently and $M M P-11,-13$ and -14 mRNAs were undetectable.

\section{Genome-wide mRNA expression studies}

In 2001, Lawrance et al. were one of the first to investigate $M M P$ expression in mucosal biopsies from IBD patients with the use of whole-genome gene expression microarrays. They found a 16-fold and 3-fold increase of MMP-12 mRNA expression in UC and CD patients, respectively, compared to healthy controls (Lawrance et al, 2001). The expression of $M M P-1$, -3 and -9 mRNAs was found to be 5-fold, 4-fold and 6-fold increased, respectively, in UC patients. A few years later, mRNA expression of $M M P-1$ and -12 was found to be elevated 3fold in both UC and CD patients compared with healthy controls (Dooley et al, 2004). Furthermore, a modest 2-fold increase of $M M P-2$ mRNA expression was found in mucosal biopsies from both CD and UC patients versus healthy controls (Costello et al, 2005). Moreover, $M M P-3$ and -7 mRNA expression was found to be 2-fold increased in UC patients compared to healthy controls and 11-fold and 7-fold increased, respectively, in inflamed UC tissue compared to inflamed control tissue. In inflamed UC tissue versus non-inflamed UC tissue, $M M P-3$ and 7 mRNA expression was 8-fold and 6-fold increased, respectively (Noble et al, 2008). In CD 
patients, $M M P-3$ mRNA expression was 7-fold upregulated in terminal ileum biopsies and MMP-1 mRNA expression was 4-fold downregulated in colon biopsies (Noble et al, 2010). A significantly higher expression of $M M P-9$ was found in UC patients with severely active disease compared with healthy controls (Lakatos et al, 2011). With the use of a human NO gene array, MMP-1 mRNA expression was found to be significantly increased in biopsies from both UC and CD patients and MMP-7 mRNA expression was significantly increased in UC patients (Gillberg et al, 2012). Finally, the expression of $M M P-1,-3,-7$ and -10 mRNAs was found to be significantly increased in the colon of pediatric UC patients with active disease compared with controls (Jimbo et al, 2014).

Since information regarding mRNA expression of all MMPs $(n=23)$ was fragmented, we performed microarray analysis on ileal and colonic mucosal biopsies from both UC and CD patients before and after treatment with infliximab (de Bruyn et al, 2014b). We found that mRNA expression levels of $M M P-1,-2,-3,-7,-9,-10,-12$ and -19 , were more than 2-fold increased, whereas MMP-28 mRNA expression was more than 2-fold decreased in inflamed colon of IBD patients as compared with colon from healthy controls. After treatment with infliximab, most of the dysregulated $M M P$ gene expression levels decreased more than 2-fold in patients who responded to treatment when compared with baseline, and $M M P-28$ expression was more than 2-fold significantly increased. In CD patients with ileal disease, the mRNA expression levels of $M M P-1,-3,-7$ and -10 were more than 2-fold significantly upregulated as compared with control ileums. After treatment with infliximab, mRNA expression levels of $M M P-1,-3,-7$ and -10 normalized in CD patients with ileal disease who responded to treatment, whereas the expression levels of $M M P-1$ and -3 remained more than 2-fold significantly upregulated in CD patients with ileal disease who did not respond to treatment. The microarray data were confirmed with qRT-PCR for $M M P-1$ and -28 . 
In the search for markers for differential diagnosis of IBD patients, Von Stein et al. first compared gene expression in inflamed and non-inflamed colorectal mucosal biopsy samples with the use of subtractive suppression hybridization (SSH) technology (von Stein et al, 2008). They identified seven marker genes (including MMP-7, SLC6A14, SLC26A2,GRO- $\alpha$, SPAP, RegIV and Vanin-1) that were differentially expressed in IBD, making it possible to discriminate between patients suffering from UC, CD or irritable bowel syndrome (IBS). A few years later, Sipos and colleagues investigated transcriptional profiles in both blood and biopsy samples (Sipos et al, 2011). The goal was similar as that from Von Stein and colleagues, namely to identify markers to differentially diagnose IBD. However, instead of looking at only the diseased colon, these authors also measured profiles in peripheral blood samples. Differences for a set of 13 transcripts (including $M M P-9$ ) were found between IBD, non-IBD and healthy control blood samples. More specifically, 16 transcripts were different for UC, non-IBD and control samples, whereas 4 transcripts were found to discriminate CD, non-IBD and control blood samples. UC and CD blood samples could be distinguished from each other by 5 genes (LOC149832, SLC24A3, DAB2, GAB2 and MSH2) with $100 \%$ specificity and sensitivity. In biopsies, IBD and control tissues could be distinguished by 3 transcripts (including $M M P-3$, REGIA and CHI3L1) with $100 \%$ specificity and sensitivity. Moreover, MMP-3, together with 3 other transcripts (CHI3L1, SLC6A14 and REG1A), was differentially expressed in UC and control tissues, whereas no $M M P$ was involved in the discrimination between $\mathrm{CD}$ and normal tissues. Differences between UC and CD tissues could be determined by a set of 58 transcripts with $100 \%$ sensitivity and $77.78 \%$ specificity. Some disease-associated alterations in blood transcripts were also detected in colonic tissue. The authors concluded that screening for differential gene expression in peripheral blood may discriminate IBD subtypes from non-IBD, including diverticulitis, infectious and ischemic colitis. 


\section{Targeted mRNA expression studies}

In mucosal biopsies from $\mathrm{UC}$ and $\mathrm{CD}$ patients, $M M P-3$ was found to be markedly overexpressed in inflamed sites. Moreover, elevated $M M P-3$ mRNA levels in CD patients with active disease returned to normal following treatment with enteral nutrition (Heuschkel et al, 2000). In inflamed colon samples from patients with $\mathrm{UC}$ and $\mathrm{CD}, M M P-1$ and -3 mRNA expression was described to be more than 15-fold increased compared with normal colon samples and the levels correlated well with the histological degree of acute inflammation (von Lampe et al, 2000). The expression of $M M P-2$ and -14 mRNAs was found to be marginally increased in inflamed, but 9-12-fold increased in ulcerated colonic mucosa of IBD patients. A moderate 2-fold upregulation of $M M P-2$ mRNA was found in mucosal tissue from UC and CD patients compared with control tissue (Gao et al, 2005). No difference in MMP-2 mRNA expression was observed between inflamed or non-inflamed tissue, whereas $M M P-9$ mRNA expression was 2-fold increased in non-inflamed and 4-fold increased in inflamed tissue compared to control tissue (Gao et al, 2005). In UC patients, the expression of MMP-1 mRNA was shown to be upregulated in inflamed and ulcerated colonic tissue and correlated with the severity of clinical symptoms (Wang \& Yan, 2006). In a follow-up study, $M M P-1$ colonic mRNA levels were found to correlate with disease severity in UC patients (Wang \& Mao, 2007). In biopsy specimens of both CD and UC patients, significantly increased levels of $M M P-2,-7$, and -13 mRNA were found in inflamed IBD mucosa compared with healthy tissue (Rath et al, 2006). However, MMP-9 and -14 mRNA levels were not significantly different between healthy and inflamed IBD mucosa. MMP-3 mRNA levels were found to be increased in affected areas in biopsies from patients with UC, but not CD, as compared to healthy controls (Leon et al, 2009). The expression of MMP-7, -13 and -28 mRNA in mucosal biopsies from affected and healthy tissues from Norwegian UC patients was further investigated by Rath and colleagues (Rath et al, 2010). MMP-7 and -13 mRNA expression was found to be significantly increased in UC 
affected colonic mucosa, whereas $M M P-28$ mRNA expression was found to be decreased in inflamed mucosa. This decrease in $M M P-28$ mRNA expression in patients with colonic inflammation was also observed in our microarray study (de Bruyn et al, 2014b). In pediatric CD patients, $M M P-2$ mRNA expression was shown to be upregulated (Sim et al, 2012). In patients with pouchitis and active UC, increased $M M P-1$ and -2 mRNA expression levels were observed in mucosal biopsies (Stallmach et al, 2000). Moreover, in patients with pouchitis compared with irritable pouch syndrome, the expression of $M M P-1 \mathrm{mRNA}$ was also found to be elevated (Schmidt et al, 2007).

Bjerrum and colleagues aimed to validate previous findings regarding a multigene study indicating that the expression of a panel of seven specific genes (including $M M P-7$ ) had strong differential power regarding IBD versus non-IBD, as well as UC versus CD (von Stein et al, 2008). However, in their prospective study they were unable to confirm that $M M P-7$, as part of the 7 gene panel, was a reasonable parameter to discriminate UC from CD and the authors suggested that the gene panel could only discriminate inflamed from non-inflamed tissue (Bjerrum et al, 2014).

\section{Cellular mRNA localization studies}

In Figure 4, cellular localization of $M M P$ mRNA expression is symbolized. Saarialho-Kere and colleagues performed in situ hybridization (ISH) on ileal and colonic samples from IBD patients and found that $M M P-1$ and -3 mRNAs were detected in considerable amounts in granulation tissue (Saarialho-Kere et al, 1996). MMP-7 mRNA was detected in damaged epithelium bordering the ulcer area, whereas no signal was detected in intact mucosal epithelium or in any stromal or inflammatory cells (Saarialho-Kere et al, 1996). Two years later, Vaalamo and colleagues evaluated the cellular expression of $M M P-10,-12$ and -13 mRNAs in intestinal samples from UC and CD patients (Vaalamo et al, 1998). They found that MMP-10 mRNA was mainly expressed in enterocytes bordering ulcerations. $M M P-12$ mRNA was found to be 
abundantly expressed by macrophages in the vicinity of shedding mucosal epithelium and beneath the necrotic surface of the ulcers. $M M P-13$ mRNA was expressed by fibroblast-like cells deeper in the remodeling intestinal wall (Vaalamo et al, 1998). Furthermore, $M M P-1$ and -2 mRNA levels were found to be markedly increased in cells of the lamina propria, especially in spindle shaped cells, predominantly in the subepithelial myofibroblasts sheet. The intensity of the $M M P-1$ and -2 mRNA signals was positively correlated with the cellular density of the inflammatory infiltrate and was significantly increased in inflamed pouches (Stallmach et al, 2000). Kirkegaard et al. investigated the cellular expression pattern of $M M P-1,-2,-3,-7,-9$ and -10 mRNAs in intestinal specimens from CD patients (Kirkegaard et al, 2004). MMP-3 mRNA was localized in large mononuclear cells and fibroblasts, whereas MMP-9 mRNA was expressed in granulocytes and only in fistulae with acute inflammation. Overall, the expression of MMP-3 and MMP-9 mRNA was found to be markedly upregulated in intestinal fistulae. MMP-2 mRNA was observed in endothelial cells and in large mononuclear macrophage-like cells and was found to be equally expressed in normal colon and fistulae.

\subsection{MMP protein expression studies}

Under normal conditions, only a selected number of MMPs is expressed at detectable levels in the gut epithelium (MMP-2, -3, -7 and -28), in resident immune cells in the lamina propria (MMP-3) and in mesenchymal cells (MMP-2, -3) (Kirkegaard et al, 2004; O'Shea \& Smith, 2014; Ravi et al, 2007). Already during the discovery phase of MMP proteins (between the years 1987 and 2000) it was recognized that some of these were induced by cytokines and chemokines, whereas others were found to be rather constitutively expressed. The expression of MMPs has been shown to be dysregulated after various forms of injury or inflammatory stimuli including TNF- $\alpha$ (Ito et al, 1990; Kim \& Lee, 2005; Pedersen et al, 2009), interleukin$1 \beta$ (Gan et al, 2001; Nee et al, 2004; Opdenakker et al, 1991; Sternlicht \& Werb, 2001; Van Ranst et al, 1991), bacterial antigens (e.g. LPS, damage- and pathogen-associated molecular 
patterns) (Kim \& Koh, 2000; Lai et al, 2003; Lopez-Boado et al, 2000; Masure et al, 1991; Opdenakker et al, 1991; Rhee et al, 2007; Van Ranst et al, 1991), colony stimulating factors (Gutschalk et al, 2013; Tomita et al, 2000), epidermal growth factor and transforming growth factor- $\beta$ (TGF- $\beta$ ) (Edwards et al, 1987; Kim et al, 2004; Yan \& Boyd, 2007).

A wide range of cell types have been shown to express MMPs, including epithelial cells, mesenchymal cells and leukocytes (Kirkegaard et al, 2004; Kruidenier et al, 2006; Matsuno et al, 2003; McKaig et al, 2003; O'Sullivan et al, 2015; Pedersen et al, 2009; Saarialho-Kere et al, 1996; Vaalamo et al, 1998; von Lampe et al, 2000). Moreover, Ravi et al. have elaborately studied the expression of MMPs in IBD and described the cellular source and function of MMPs in normal conditions and in IBD (Ravi et al, 2007). In summary, studies in which MMP protein expression were investigated are listed in Table $\mathbf{4}$ and MMP mucosal expression patterns are symbolized in Figure 4.

\section{In vitro/ex vivo protein expression studies}

\section{Cell lines}

In a human intestinal epithelial cell line (Caco-2), the expression of MMP-9 after stimulation with IL-1 $\beta$ and TNF- $\alpha$ was evaluated with ELISA (Gan et al, 2001). Stimulation of the Caco-2 cells with both agents led to a dose-dependent increase in MMP-9 secretion. In contrast, LPS failed to directly or indirectly (after IL-1 $\beta$ or TNF- $\alpha$ induction) induce secretion of MMP-9. In addition, treatment with rosiglitazone, a selective PPAR- $\gamma$ activator, significantly reduced MMP-9 production in Caco-2 cells. Furthermore, Claramunt and colleagues investigated the effect of N-unsubstituted curcuminoid pyrazoles on MMP-2 and -9 expression in Caco-2 cells (Claramunt et al, 2009). Three compounds were shown to significantly decrease MMP-9 activity in inflammation-induced Caco-2 cells and thought to be reasonable candidates for treatment of IBD patients. In a T84 epithelial cell line, the role of MMP-7 was investigated in the process of wound healing (Puthenedam et al, 2011). MMP-7 was shown to cleave galectin- 
3 resulting in three cleaved fragments. Therefore, cleavage of galectin-3 may be one mechanism by which MMP-7 inhibits wound healing. This study has significance in understanding delayed wound healing in chronic intestinal diseases like intestinal ulcers and IBD, where MMP-7 protein expression is elevated with a decreased galectin-3 protein expression as a result. Interestingly, Ailenberg and colleagues investigated the expression of MMP-2 related to intestinal barrier function in IBD (Ailenberg \& Sefton, 2009). They employed a novel matrix metalloproteinase sequestering biomaterial (MI TheramerTM beads) which restored the epithelial barrier in a double chamber in vitro test system after disruption by Cytochalasin D and activation of MMP-2. This study highlights a potential benefit for local (as opposed to systemic) inhibition of MMPs in IBD or other intestinal inflammatory diseases.

\section{Cultured primary cells}

Pender and colleagues evaluated the expression of MMP-1, -2, -3, -9 and -14 in a human fetal mesenchymal cell line (Pender et al, 2000). Mesenchymal cells cultured in serum-free media produced basal levels of MMP-1, $-2,-3,-14$. The ligation of $\alpha 4 \beta 1$ integrin with an activating antibody or a VCAM-1 fusion protein was shown to facilitate mesenchymal cell invasion and migration through the extracellular matrix. This process was mediated by an upregulation of MMP-14 and production of activated MMP-2 and could be inhibited by TIMP-2. Latent forms of MMP-1 and -3 were found to be expressed by all myofibroblasts cultures obtained from resection specimens of active UC and fibrotic CD patients (McKaig et al, 2003). With gelatin zymography, it was shown that MMP-2 was expressed in both activated and latent forms in the myofibroblasts cultures, whereas no MMP-9 expression was observed. Stimulation of an intestinal fibroblast culture with IL-21 dose-dependently enhanced the secretion of active and inactive forms of MMP-1, -2, -3 and -9 (Monteleone et al, 2006). Moreover, co-stimulation of the fibroblast cultures with IL-21 and TNF- $\alpha$ was shown to produce more MMPs than either stimulation alone. To further confirm IL-21 regulation of MMP secretion, fibroblasts stimulated 
with CD lamina propria mononuclear cell (LPMC) supernatant were shown to enhance the production of MMPs and subsequent addition of a blocking IL-21 receptor fusion protein (IL21R/Fc) resulted in a decrease of MMP production.

Curcumin has previously been described to maintain remission in patients with quiescent UC (Hanai et al, 2006). Therefore, Epstein and colleagues investigated the effect of addition of curcumin to cultures of colonic mucosal biopsy and myofibroblasts from children and adults with active IBD. They found that curcumin led to a decrease in MMP-3 production in colonic myofibroblasts in a dose-dependent way (Epstein et al, 2010). Vitamin D has also been described to have beneficial effects in IBD (Mouli \& Ananthakrishnan, 2014). The effect of vitamin D analogue ZK 156979 on MMP-2 and -9 expression was evaluated in a culture of PBMCs (Martinesi et al, 2010). MMP-2 and -9 expression was increased in PBMCs of healthy controls, UC and CD patients after addition of polyhydroxyalkanoates (PHA), LPS or TNF- $\alpha$. Subsequent addition of the vitamin D analogue ZK 156979 led to a decrease in MMP-9 levels. In contrast, MMP-2 levels only decreased after combination of ZK 156979 with anti-TNF. These data suggest a specific down-regulation of MMP-9 expression by the vitamin D analogue ZK 156979. Mucosal plasma cells isolated from UC and CD patients were shown to express significantly higher MMP-3 levels than plasma cells isolated from healthy controls (Gordon et al, 2008). The majority of MMP-3-expressing plasma cells were shown to be immunoglobulin $\mathrm{G}$ (IgG) positive. Moreover, in a LPMC culture isolated from UC and CD patients, phosphodiesterase 4 (PDE4) inhibitor CC-10004 significantly inhibited MMP-3 expression in a dose-dependent manner, whereas MMP-3 expression in cultured myofibroblasts was not altered (Gordon et al, 2009).

\section{Organ and tissue explants}

In a culture of human fetal small intestine, the expression of MMP-1, $-2,-3$ and -9 was evaluated by Pender et al. (Pender et al, 1997). With ELISA, a 3-fold increase of MMP-1, 10-fold increase 
in MMP-3, no increase in MMP-2 and no detection of MMP-9 (below limit) were found in T cell stimulated explants compared to control explants. With Western blot analysis, activated forms of MMP-1 and MMP-3 were found in organ culture supernatants of T cell-stimulated explants, whereas no increase in MMP-2 was observed. Moreover, an increase in proMMP-9 levels was seen in the explant supernatants, but no activated enzyme was detected. An increased concentration of proMMP-9 and activated and latent MMP-2 was also detected with gelatin zymography. Immunohistochemistry for MMP-1 and -3 on frozen sections of T cell-stimulated explants showed diffuse staining throughout the subepithelial layer. A year later, the same authors investigated the effect of TNFR-IgG on MMP expression in T cell-stimulated organ cultures of human fetal small intestine (Pender et al, 1998). In control supernatants, MMP-1, 2, -3 and -9 levels were detected. After T cell activation, increased amounts of both the latent and activated forms of MMP-1 and -3 and increased amounts of latent forms of MMP-2 and -9 were detected. Treatment with TNFR-IgG resulted in a marked reduction of MMP-3 and a slight decrease in MMP-1 and -9, whereas it had little effect on MMP-2 protein levels. Expression of MMP-1, -2, -3 and -9 in basal medium of intestinal explants was found to be higher in IBD versus control explants (Meijer et al, 2007a). Within the IBD group, UC compared to CD explants expressed higher MMP levels. After T cell stimulation of the explants, MMP-2 levels were lower compared to basal supernatants, whereas MMP-1, -3, and -9 levels were increased. Incubation with infliximab did not affect basal and T cell-stimulated MMP-2 secretion, whereas secretion of MMP-1, -3, and -9 was significantly decreased. In an organ culture of colonic mucosa from CD and UC patients, MMP-3 was either undetectable or below the sensitivity in the majority of uninflamed samples. In contrast, MMP-3 levels were significantly increased in inflamed mucosa from both CD and UC patients (Louis et al, 2000). Moreover, MMP-3 levels correlated with the release of pro-inflammatory cytokines and anti-inflammatory IL-10 as well as with the degree of macroscopic and microscopic inflammation. 


\section{Cellular protein localization studies}

Cellular localization of MMP protein expression is symbolized in Figure 4. Below, the localization of individual MMP expression in adult IBD tissue is discussed, followed by separate paragraphs on MMP expression and localization in pediatric IBD tissue and changes in MMP expression and/or localization after treatment.

\section{MMP-1}

In mucosal biopsies obtained from UC and CD patients, MMP-1 signal was observed in isolated mononuclear cells and no difference was found between normal or inflamed tissue (Bailey et al, 1994). A few years later, a positive staining of MMP-1 in mononuclear cells was confirmed in normal colon from UC and CD patients and these were identified as macrophages (von Lampe et al, 2000). In inflamed tissue, the number of positive staining mononuclear cells increased slightly, but with a patchy distribution. Moreover, aggregates of cells staining positive for MMP-1 were observed in severe inflamed tissue. MMP-1 staining was also detected along the basal membrane of small blood vessels within the inflamed tissue, whereas this was not observed in non-inflamed tissues (von Lampe et al, 2000). Cellular expression of MMP-1 was also investigated by our group, in validation of the microarray data (de Bruyn et al, 2014b). We found that MMP-1 was expressed in the cytoplasm of primitive cells at the base of the crypts in normal mucosa, whereas in inflamed IBD mucosa, the expression of MMP-1 shifted toward immature and surface epithelial cells according to the regeneration of the epithelial layer. Moreover, MMP-1 expression was also found in endothelial cells in areas of active inflammation. In addition, MMP-1 was found to be expressed in inflammatory cells as well as fibroblasts, most prominently in actively inflamed areas in ulcer bases and to a lesser extent in intact inflamed mucosa (Arihiro et al, 2001). In line with the previous study by Von Lampe et al. (von Lampe et al, 2000), vascular smooth muscle cells were shown to express MMP-1. Moreover, MMP-1 was found to be expressed in stromal cells (Matsuno et al, 2003). In normal 
colonic tissue and fistulae from CD patients with fistulizing disease, the cellular expression of MMP-1 was found to be low (Kirkegaard et al, 2004). In colonic tissue from CD patients, MMP1 appeared to be more strongly expressed predominantly around mucosal glands compared to control tissue (Stumpf et al, 2005).

\section{MMP-2}

In an early study by Bailey et al., no signal was found for MMP-2 in inflamed or normal tissue obtained from UC and CD patients (Bailey et al, 1994). Almost a decade later, MMP-2 was found to be expressed in stromal cells in UC colonic tissue (Matsuno et al, 2003). Moreover, MMP-2 was found to be equally expressed in normal colon and fistulae from CD patients with fistulizing disease (Kirkegaard et al, 2004).

\section{MMP-3}

A bright signal for MMP-3 was observed in a small number of mononuclear cells in normal CD tissue, with only a slight increase of numbers of cells in inflamed CD tissue (Bailey et al, 1994). Interestingly, in tissue sections from patients with UC, MMP-3 was detected in regions of mucosal damage, but was not observed in normal tissue. In confirmation of the latter study, a positive staining of MMP-3 was found in normal colon from UC and CD patients in a few mononuclear cells and these were identified as macrophages (von Lampe et al, 2000). In addition, the cellular expression of MMP-3 in mucosal tissue of both UC and CD patients was found in inflammatory cells as well as in fibroblasts, most prominently in actively inflamed areas in ulcer bases and to a lesser extent in intact inflamed mucosa (Arihiro et al, 2001). In line with the previous study, MMP-3 was found to be localized in large mononuclear cells and fibroblasts in mucosal tissue from CD patients with fistulizing disease (Kirkegaard et al, 2004).

\section{MMP-7}

In IBD tissue and gastrointestinal ulcers, MMP-7 was detected in the epithelium bordering areas where neutrophils associated with the disrupted epithelium, particularly in cryptitis or crypt 
abscesses (Saarialho-Kere et al, 1996). No signal was detected in intact mucosal epithelium further away from the ulcers or in any stromal or inflammatory cells, suggesting that MMP-7 is not involved in the regular epithelial renewal, but participates in epithelial regeneration. The cellular expression of MMP-7 was low in mucosal tissue from CD patients with fistulizing disease (Kirkegaard et al, 2004). In mucosal biopsies from UC patients, MMP-7 was expressed in inflammatory and endothelial cells suggesting a role in both colitis-associated neoangiogenesis and inflammatory changes (Rath et al, 2010).

\section{MMP-8}

The cellular expression of MMP-8 in mucosal tissue of both UC and CD patients was found in inflammatory cells as well as in fibroblasts, most prominently in actively inflamed areas in ulcer bases and to a lesser extent in intact inflamed mucosa (Arihiro et al, 2001).

\section{MMP-9}

A bright signal for MMP-9 was found in a few isolated polymorphonuclear leukocytes (PMNLs) throughout the wall of the intestine in normal tissue, whereas large numbers of brightly stained PMNLs were found in inflamed tissue mainly in the lamina propria, submucosa and a few in the muscularis propria (Bailey et al, 1994). These data are in line with the fact that neutrophils produce and store MMP-9 in various proforms (vide infra) in tertiary granules, thus yielding bright signals upon immunohistopathological analysis (Opdenakker et al, 2001a). Most acute inflammations in humans lead to the production of IL-8 attracting neutrophils (Van Damme et al, 1988) and inducing MMP-9 release from neutrophils (Masure et al, 1991). As a consequence, immunodetection of MMP-9 in degranulated PMNLs is less bright. In confirmation with the study by Bailey et al., MMP-9 staining was found in the lamina propria consistent with the distribution of infiltrating neutrophils in inflamed connective tissue from UC and CD patients (Baugh et al, 1999). The expression pattern of MMP-9 was further investigated in patients with UC, lymphocytic colitis (LC) and collagenous colitis (CC) 
(Lakatos et al, 2011). MMP-9 was mainly expressed within the cytoplasm and cytoplasmic membranes of colonic epithelial cells. The prominent location of MMP-9 was in the submucosa with positive staining in PMNLs as well as a weak positive signal in the lamina propria with increased intensity according to severity of the inflammation in UC tissues. Occasionally, MMP-9 positive macrophages were also found, predominantly in inflamed areas. These data are in line with the fact that macrophages and other mononuclear cells need to synthesize MMP9 de novo after stimulation with pro-inflammatory cytokines (Opdenakker et al, 2001a). No other major cell types showed MMP-9 immunoreactivity in normal tissues. Moreover, significantly more MMP-9 immunoreactivity was found in stromal inflammatory cells of the colon of UC patients when compared to the samples from the non-IBD patients (Piekkala et al, 2013). In addition, MMP-9 immunoreactivity correlated with the histological grade of inflammation, but not with the other markers of active disease. In the search for a peripheral blood test to differentially diagnose IBD patients, Sipos et al. investigated MMP-9 expression in blood smears and biopsies from UC and CD patients. They found more MMP-9 positive cells in blood smears and biopsies of UC and CD patients with active disease than in normal samples or non-IBD controls (Sipos et al, 2011). The cellular expression of MMP-9 in mucosal tissue of both UC and CD patients was found in inflammatory cells as well as in fibroblasts, most prominently in actively inflamed areas in ulcer bases and to a lesser extent in intact inflamed mucosa (Arihiro et al, 2001). Vascular smooth muscle cells were also shown to express MMP9. Moreover, in mucosal tissue from UC patients, MMP-9 was found to be expressed in stromal cells (Matsuno et al, 2003) and in mucosal tissue from CD patients with fistulizing disease, MMP-9 was expressed in granulocytes and only in fistulae with acute inflammation (Kirkegaard et al, 2004).

MMP-10 
MMP-10 was found to be localized at the edge of intestinal epithelium bordering the ulcers which suggested that MMP-10 plays a role in epithelial migration (Vaalamo et al, 1998). In addition, MMP-10 staining was negative in mucosal tissue from CD patients with fistulizing disease (Kirkegaard et al, 2004).

\section{MMP-13}

In colonic tissue from CD patients, MMP-13 staining was observed in only a few tissues and no differences could be observed between CD and control tissue (Stumpf et al, 2005). The expression of MMP-13 was further investigated by Vizoso et al. in mucosal biopsies from UC and CD patients (Vizoso et al, 2006). More than $60 \%$ of patients with UC and $50 \%$ of patients with CD had MMP-13 expression in inflamed tissue. The expression was found to be highest in ulcerated colonic mucosa and a positive correlation was found between MMP-13 expression and histological inflammation scores. No MMP-13 expression was detected in uninflamed tissue, in control colon mucosa or in colonic mucosa from patients with acute diverticulitis. In addition, MMP-13 was found to be expressed in inflammatory and endothelial cells in UC patients suggesting a role in both colitis-associated neoangiogenesis and inflammatory changes.(Rath et al, 2010).

\section{MMP-19, -26 and -28}

Bister and colleagues were the first to evaluate the cellular expression pattern of MMP-19, MMP-26, and MMP-28 (Bister et al, 2004). All three MMPs were shown to be expressed in normal intestine. In inflamed tissue, MMP-19 was expressed in non-migrating enterocytes and shedding epithelium and MMP-26 was detected in migrating enterocytes. In contrast, MMP-28 was not associated with inflammatory and destructive changes seen in IBD. Furthermore, the expression of MMP-28 was found to be decreased in UC and was linked to colitis-associated epithelial destruction and loss of cryptal architecture (Rath et al, 2010). The latter finding about 
decrease of MMP-28 protein is in line with our microarray mRNA expression data (de Bruyn et al, 2014b).

\section{Pediatric IBD patients}

The cellular expression of MMP-1, -7, -8, -9, -10, -12 and -26 was further investigated in pediatric IBD patients (Makitalo et al, 2010). The expression of MMP-7 in colonic epithelium was higher in CD samples compared with UC samples (Makitalo et al, 2010). MMP-10 expression was elevated in the epithelium of both CD and UC samples compared with non-IBD samples (Makitalo et al, 2010). The authors suggested that increased expression of epithelial MMP-10 could serve as a histological indicator of IBD etiology (Makitalo et al, 2010), whereas epithelial MMP-7 expression may help to differentiate between CD-related colitis and UC (Makitalo et al, 2010). Moreover, in pediatric UC patients with a pouch, most pouch samples showed expression of epithelial and stromal MMP-3, -7, and -12, but MMP-8, -9 and -26 expression was lacking (Makitalo et al, 2012a). In samples with low grade inflammatory activity, the epithelial MMP-3 and -7 expression was increased. MMP levels did not correlate with fecal calprotectin amounts, erythrocyte sedimentation rate or CRP levels. Furthermore, the expression of MMP-1, $-3,-7$, and -10 proteins was found to be enhanced in epithelial cells and lamina propria in mucosal colon samples from pediatric IBD patients without differences between UC or CD patients (Jimbo et al, 2014).

\section{Changes in MMP expression and/or localization after treatment}

Geboes et al. investigated the long-term effect of anti-TNF treatment (infliximab) on endoscopic and histologic disease activity and expression of gelatinase B (MMP-9) in CD patients who were enrolled in the ACCENT 1 study (Geboes et al, 2005). A sustained reduction in the cellular expression of MMP-9 was associated with endoscopic and histologic evidence of mucosal healing. The cellular localization of MMP-2 and -9 was further investigated by Gao et al. in UC and CD patients (Gao et al, 2005). MMP-2 was found to be most prominently 
present in the ECM of the submucosa, whereas a weak diffuse cytoplasmic staining for MMP2 was present in some epithelial cells. MMP-9 had a similar pattern in the intestinal mucosa as MMP-2 and was mostly present in PMNLs throughout the intestinal layers. A few years later, the influence of infliximab on the expression of MMP-2 and -9 in mucosal biopsies from CD patients was investigated (Gao et al, 2007). MMP-2 levels did not clearly change, whereas MMP-9 expression in PMNLs was decreased after treatment with infliximab. These data confirm the results obtained by Geboes and colleagues (Geboes et al, 2005). The expression of MMP-1, -7, -9, -10, and -26 after immunosuppressive treatment was further investigated in CD patients (Makitalo et al, 2009). MMP-7, -9 and -26 decreased along with treatment (infliximab, adalimumab, CS, AZA or MTX), whereas expression of MMP-1 and -10 was not altered. A positive correlation was found between the total histological score and neutrophil-derived MMP-9 and -26 levels. Moreover, the expression of calprotectin seemed to have a similar pattern compared with stromal MMP-26 expression. 


\section{Protein quantification studies}

\section{Mucosal biopsies}

In mucosal tissues from pediatric and adult UC and CD patients, the protein expression levels of MMP-1, -2, -3 and -9 were investigated by Baugh and colleagues (Baugh et al, 1999). With gelatin zymography, a significant increase in proteolysis was observed in tissues from UC patients compared with control tissue and more proteolytic activity was present in inflamed tissue compared to non-inflamed tissue. Importantly, gelatinolytic levels correlated with endoscopic and histological scores. In CD patients, no significant differences were observed between inflamed and non-inflamed tissue, although increased proteolysis was observed in ulcerated and non-ulcerated sites versus controls. With Western blot, it was shown that proMMP-9 as well as the activated form of MMP-9 were abundantly present in inflamed tissue. In contrast, MMP-1 was weakly expressed in $\mathrm{UC}, \mathrm{CD}$ and control tissue with little variation. MMP-2 and -3 were not routinely detected in UC, CD or control tissue, although in some of the inflamed UC and CD tissues the activated form of MMP-3 was detected. The expression of MMP-1, -2 and -3 in UC and CD patients was also evaluated by Von Lampe and colleagues (von Lampe et al, 2000). They found an increased expression of MMP-1, -2, and -3 in lysates from inflamed colonic mucosal tissue compared with non-inflamed colonic tissue from the same patients. The most intense bands on Western blot for all three MMPs were in the latent (inactive) form.

The expression of MMP-7, -13 and -28 was further evaluated with ELISA (Rath et al, 2010). MMP-7 was significantly increased in tissue from UC patients compared with unaffected tissue, whereas MMP-13 levels were below the limit of detection in all samples. Moreover, MMP-7 or -13 levels were not associated with histopathological grading or endoscopic classification of inflammation. In a study by Meijer et al., the expression and activity of MMP-1, -2, -3 and -9 were evaluated with ELISA and specific immunosorbent bioactivity assays, respectively, in 
homogenized colonic/ileal tissue from UC and CD patients (Meijer et al, 2007a). They found an increased expression of MMP-1, $-2,-3$ and -9 relative to TIMP-1 and -2 in inflamed tissue compared to non-inflamed and control tissue. Moreover, net MMP-1, $-2,-3$ and -9 activity was increased in inflamed tissue, with similar expression profiles in CD and UC. The association between upregulated levels of MMP-1, -2, -3, -9 and morphological damage described by previous studies was hereby confirmed, not only at the MMP protein level, but particularly at the activity level. The authors therefore concluded that further research should focus on proof of in vivo activity of MMPs. In mucosal tissue from pediatric UC and CD patients, MMP-3 was found to be markedly elevated in inflamed tissue of IBD patients with active disease, but was not elevated in adjacent endoscopic normal mucosa (Heuschkel et al, 2000). In UC patients with pouchitis, MMP-1 and -2 levels were shown to be increased in mucosal tissue compared with UC patients and familial adenomatous polyposis (FAP) patients without pouchitis (Stallmach et al, 2000). Furthermore, both MMPs were significantly increased in inflamed pouches compared with non-inflamed ileal mucosa. Increased MMP-1 and -2 levels were comparable between UC patients with pouchitis and UC patients with active disease. Treatment of patients with pouchitis with metronidazole resulted in a significant decrease of MMP-1 and -2 levels after improvement of clinical symptoms and endoscopic/histological signs of pouchitis. With the use of Western blot analysis, it was further shown that only in biopsies from patients with pouchitis activated forms of MMP-1 and -2 were found. Finally, the effect of vitamin D derivatives on adhesion molecules and MMPs was investigated in colonic biopsies of IBD patients (Martinesi et al, 2014). The expression of MMP-2, -3, and -9 was decreased in biopsies after addition of vitamin $\mathrm{D}$ derivatives in $\mathrm{UC}$ and $\mathrm{CD}$ with the exception of $1,25(\mathrm{OH}) 2 \mathrm{D} 3$ that did not affect MMP-9 and -2 levels in CD.

The expression of MMP-2, -3 and -9 was also investigated with gelatin zymography and immunohistochemistry in mucosal tissue from CD patients with fistulizing disease (Kirkegaard 
et al, 2004). MMP-3 expression was increased in fistulae compared with normal colon, irrespective of the stage of inflammatory activity. Moreover, activated forms of MMP-2 and -9 were found in fistulae. Gelatinase A and B (MMP-2 and MMP-9, respectively) levels were further investigated in UC and CD patients (Gao et al, 2005). MMP-2 levels measured with ELISA were similar in tissues of CD and UC patients, in both inflamed and non-inflamed tissues, but were significantly higher than in the control tissues. With zymography, it was shown that the activated and the pro-enzyme form of MMP-2 were increased in IBD without major differences between inflamed and non-inflamed tissues and between $\mathrm{CD}$ and UC. In contrast, with the use of highly specific biochemical immunosorbent activity assays (BIA), the authors showed that the biochemical activity of MMP-2 was not increased in IBD tissue. In addition, MMP-9 levels were found to be increased 4-fold in non-inflamed IBD tissue and 7-fold in inflamed IBD tissue compared to control tissue. With zymography both the activated and the pro-enzyme form of MMP-9 were found to be significantly increased in IBD and predominantly pro-MMP-9 was found to increase with the degree of inflammation. Moreover, the biochemical activity of MMP-9 was found to be increased in both non-inflamed and inflamed IBD tissue. MMP-2 and -9 levels were also found to be increased in both UC and CD biopsies as measured with ELISA (Rath et al, 2006). In ischemic colitis patients, higher MMP-2 and -9 expression was found in affected colonic mucosa as measured by zymography and Western blot (Medina et al, 2006b). The mucosal expression of MMP-9 was found to be significantly higher in patients with UC compared to patients with lymphocytic colitis (LC), collagenous colitis (CC), active diverticulitis, inactive diverticular disease and controls (Lakatos et al, 2011). Moreover, MMP9 levels correlated with the severity of inflammation in patients with UC. Therefore, increased MMP-9 expression could contribute to the severity of mucosal damage in UC patients with active disease. In our microarray study, we performed additional experiments to confirm MMP mRNA expression at the protein level (de Bruyn et al, 2014b). We investigated mucosal MMP- 
2 and -9 protein levels with the use of gelatin zymography. We did not observe activated MMP9 or NGAL-MMP-9 levels in control tissues, whereas these were abundantly present in inflamed tissues from IBD patients (Figure 5, top panel). In several cited studies, gelatin zymography was used, but misinterpreted. Gelatin zymography is a technique to detect the presence of gelatinases. If appropriately standardized, with this technique one can even quantify the gelatinase levels. However, with gelatin zymography it is not possible to conclude that the observed enzyme is active or not. First, pro-enzyme forms of MMPs, which are inactive per se, are activated during the processing of the zymography gel. Secondly, whereas in a biological sample gelatinases maybe complexed with and completely inactivated by TIMPs, during the zymography procedure these complexes dissociate and the freed gelatinases give the impression of presence of activity. It may also be relevant to point out that activated gelatinases, having lost the propeptide sequence, have a smaller molecular weight than the proform and can be distinguished by gelatin zymography (de Bruyn et al, 2014a; Vandooren et al, 2013a). Therefore, in addition to gelatin zymography, we used a gelatin degradation assay (Vandooren et al, 2011) in our study and observed a trend for higher gelatinolytic activity levels before start of infliximab in tissue obtained from IBD patients with active disease and a decrease of activity levels after treatment (de Bruyn et al, 2014b).

\section{Blood}

Plasma concentrations of MMP-1 were found to be significantly elevated in UC patients in comparison to healthy controls, although no correlation between MMP-1 and laboratory, clinical or endoscopic indices of disease activity were observed (Wiercinska-Drapalo et al, 2003). Both UC and CD patients had increased MMP-3 and -9 serum levels and these correlated with clinical indices, indicating their involvement in the disease activity (Silosi et al, 2014). In addition, both MMP-3 and -9 levels were found to be higher according to disease severity in the serum of pediatric UC patients (Kofla-Dlubacz et al, 2014). Moreover, MMP-3 was found 
to be the best marker to discriminate UC patients from healthy controls compared with other measured markers (MMP-9, CRP and ESR). The influence of infliximab on the expression of MMP-2 and -9 was investigated in sera from CD patients (Gao et al, 2007). The authors found that serum MMP-2 levels increased after treatment with infliximab and the increase was similar in patients who responded to treatment compared to those who did not respond. In contrast, serum MMP-9 levels were decreased particularly in patients who responded to treatment. The effect of glucocorticoid (GC) and anti-TNF therapy on MMP-7, -8, -9 expression was further evaluated in serum of IBD patients (Makitalo et al, 2012b). MMP-7, -8, -9 levels were elevated at start of treatment in IBD patients compared with controls. During GC therapy and anti-TNF therapy MMP-7 decreased, although levels remained higher after anti-TNF treatment than after GC therapy. The levels of MMP-7 did not markedly associate with inflammatory markers in blood or feces.

To investigate the use of MMP-9 as a biomarker in IBD, serum MMP-9 was measured together with other serum and fecal markers in UC and CD patients (Faubion et al, 2013). The combination of fecal calprotectin and serum MMP-9 was found to be optimal for discriminating disease activity in UC patients as measured by the Mayo Clinic endoscopy subscore. In CD, the combination of fecal calprotectin, serum MMP-9 and serum IL-22 demonstrated the strongest association with imaging/endoscopy-defined inflammation. To follow-up on our initial microarray (mRNA) study (de Bruyn et al, 2014b), we investigated the impact of infliximab treatment on gelatinase (MMP-2 and -9) protein levels in serum of UC patients (de Bruyn et al, 2014a). Of interest and not yet described in previous literature, serum NGAL-MMP-9 levels were found to be elevated in UC patients with active disease and decreased after treatment with infliximab in patients who achieved mucosal healing. Moreover, NGAL-MMP-9 correlated with the Mayo endoscopic subscore as well as histopathological score and performed better than CRP in discriminating mucosal healing. Furthermore, we found that NGAL-MMP-9 levels 
were also elevated in the serum of CD patients with active disease compared with healthy controls (de Bruyn et al, 2015). After treatment with infliximab, serum NGAL-MMP-9 levels decreased in patients who achieved mucosal healing and to a lesser extent in patients who did not show complete endoscopic healing. NGAL-MMP-9 levels correlated with endoscopic and histopathological scores and were shown to be superior compared to CRP levels for the discrimination of patients with and without mucosal healing.

\section{Urine}

In the urine of pediatric IBD patients, MMP-2 and -9 levels were significantly elevated in patients with both UC and CD compared to controls (Manfredi et al, 2008). Moreover, multiple logistic regression analysis revealed that urinary MMP-2 and NGAL-MMP-9 complex levels were independent predictors of CD and UC.

\section{Feces}

Annahazi et al. were the first to investigate fecal MMP-9 levels in UC patients (Annahazi et al, 2013). They found that MMP-9 was very low or undetectable in the feces of all controls and IBS patients. In UC patients, fecal MMP-9 levels significantly correlated with the overall Mayo score, the endoscopic score, serum CRP levels and fecal calprotectin. The authors concluded that fecal MMP-9 is a useful tool in the differential diagnosis of diarrheic disorders and in the noninvasive evaluation of disease activity and mucosal healing in UC. In a follow-up study, fecal MMP-9 levels were measured in pediatric IBD patients and the performance of MMP-9 was compared with fecal calprotectin (Kolho et al, 2014). Although calprotectin was the best fecal marker in pediatric IBD, MMP-9 showed an almost comparable performance in UC, suggesting its applicability as a surrogate marker of inflammation. Recently, fecal MMP-9 was further investigated as a biomarker in both UC and CD patients (Farkas et al, 2015). Fecal MMP-9 did not correlate with any of the activity indices of $\mathrm{CD}$, whereas in line with previous 
studies, strong associations were found between fecal MMP-9 and clinical, endoscopic and histologic activities of UC and pouchitis.

\section{ASSOCIATION STUDIES OF TIMPS IN IBD}

\subsection{TIMP genetic association studies}

Several functional SNPs have been described in TIMP genes (as reviewed by Langers et al. (Langers et al, 2011)). In an exon segment of TIMP-1 a T/C transition at position +372 was found, whereas in TIMP-2 a G/A transition at position +303 was detected. To investigate whether the presence of these SNPs was associated with IBD, Meijer et al. studied the genotype distributions at the previously discovered SNP loci of the genes encoding TIMP-1 and -2 (Meijer et al, 2007c). They tested 245 IBD patients and found that the genotype TT in women and T in men at SNP +372 T/C increased CD susceptibility. Moreover, women with the TIMP1 genotype TT were found to be less prone to the development of fistulae. Male patients carrying the +372 T-allele expressed lower levels of TIMP-1 in surgically resected inflamed tissue. In a study by Morgan et al., the association of SNPs in TIMP-1, -3 and -4 with UC was further investigated (Morgan et al, 2011). However, they did not find any significant associations of SNPs in TIMP genes with UC parameters.

\subsection{TIMP mRNA expression studies}

In a range of studies, mRNA expression of TIMPs was evaluated in IBD and these are summarized in Table 5 and cellular localization of TIMP mRNA expression is symbolized in

\section{Figure 4.}

\section{In vitro/ex vivo mRNA expression studies}

In several studies, contrasting results were obtained with regard to in vitro TIMP expression. In a culture of human fetal small intestine explants, TIMP-1 mRNA was detected in both control 
and $\mathrm{T}$ cell stimulated explants (Pender et al, 1997). Moreover, the expression of TIMP-1 transcripts was found to be significantly higher in myofibroblasts isolated from fibrotic CD tissue compared with those obtained from normal tissues (McKaig et al, 2003). In contrast, in an ex vivo model of $\mathrm{T}$ cell mediated gut injury, TIMP-2 and -4 gene expression remained unchanged, whereas the expression of TIMP-1 and -3 decreased after $\mathrm{T}$ cell stimulation (Salmela et al, 2002). Moreover, T cell derived IL-21 stimulation did not enhance TIMP-1 and -2 mRNA levels in intestinal fibroblasts isolated from CD patients (Monteleone et al, 2006). TIMP-1 mRNA levels in plasma cells isolated from colonic mucosal tissue from UC and CD patients were also not significantly different from those of control samples (Gordon et al, 2008).

\section{Genome-wide mRNA expression studies}

In one of the first microarray studies, no differences in TIMP-1 mRNA expression were found in intestinal tissues from controls or UC and CD patients (Lawrance et al, 2001). In another microarray study, TIMP-1 mRNA expression was found to be 4-fold upregulated in UC patients and 2-fold in CD patients versus healthy controls (Costello et al, 2005). Moreover, with the use of a human NO gene array, TIMP-1 mRNA expression was found to be significantly increased in biopsies from both UC and CD patients (Gillberg et al, 2012). Since the information regarding TIMP mRNA expression in the literature was limited, we performed microarrays on ileal and colonic mucosal biopsies from patients with UC and CD and evaluated gene expression of 4 TIMPs (de Bruyn et al, 2014b). The mRNA expression level of TIMP-1 and -2 was 11-fold and 2-fold increased, respectively, in colon of IBD patients with active disease compared to control colon. After treatment with infliximab, the mRNA expression levels normalized to control levels in patients who responded to treatment. In CD patients with ileal disease, the mRNA expression of TIMP-1 was 6-fold increased in inflamed ileum compared to control ileum and decreased to control levels after treatment in patients who responded to infliximab. Moreover, the increased ratio of $M M P-1 / T I M P-1$ expression at baseline in IBD patients with 
active disease was restored in patients who responded to treatment. The microarray data for TIMP-1 were confirmed with qRT-PCR (de Bruyn et al, 2014b).

\section{Targeted mRNA expression studies}

Expression of TIMP-1 mRNA was found to be marginally increased in inflamed, but to be 9to 12-fold increased in ulcerated colonic mucosa of IBD patients (von Lampe et al, 2000). Moreover, significantly higher TIMP-1 mRNA levels were found in UC patients compared with CD patients and controls (Lawrance et al, 2001). The expression of TIMP-1 mRNA in ulcerated and inflamed colonic mucosa was significantly higher than in non-inflamed colonic mucosa (Wang \& Yan, 2006). Moreover, in biopsies from patients with UC and CD, TIMP-1 mRNA levels were increased in affected areas compared with control tissues (Leon et al, 2009). In contrast, TIMP-1 mRNA was found to remain unaltered in mucosal biopsies from inflamed sites in patients with IBD (Heuschkel et al, 2000) and no statistically significant TIMP-1 mRNA expression differences were found between healthy and inflamed intestinal tissue of UC or CD patients (Rath et al, 2006).

\section{Cellular mRNA localization studies}

In Figure 4, the cellular mRNA expression of several TIMPs is symbolized. With in situ hybridization, abundant TIMP-1 mRNA expression was detected in granulation tissue at the base of the ulcer in ileal and colonic samples from IBD patients (Saarialho-Kere et al, 1996). TIMP-3 mRNA was widely expressed in the intestine by endothelial cells around blood vessels and by macrophage- or fibroblast-like cells in the lamina propria surrounding damaged crypts or ulcers, whereas epithelial cells were negative (Vaalamo et al, 1998). Moreover, TIMP-3 mRNA was also detected in normal intestine, however, fewer positive cells were found compared with inflamed intestine. 
As a conclusion, in most IBD mRNA studies, TIMP mRNA levels were increased in inflamed tissues. This is reminiscent of increased MMP mRNA levels, regulated by inflammatory stimuli.

\subsection{TIMP protein expression studies.}

In several studies, TIMP protein expression in IBD was investigated and these results are summarized in Table 6, according to the type of study. Moreover, TIMP mucosal expression patterns are symbolized in Figure 4.

\section{In vitro/ex vivo studies}

The protein expression of TIMP-1 and -2 was first evaluated in a culture of human fetal small intestine (Pender et al, 1997). No increase in TIMP-1 and -2 levels was observed in T cellstimulated explants compared to control explants. In a follow-up study, the effects of TNFRIgG on the expression of TIMP-1 were analyzed in an organ culture of human fetal small intestine and in mesenchymal cells (Pender et al, 1998). TIMP-1 levels were detected in control supernatants and the levels increased after T cell activation. Addition of TNFR-IgG had little effect on TIMP-1 levels. In isolated mucosal mesenchymal cells, TNF- $\alpha$ was shown to induce the expression of TIMP-1 and subsequent addition of TNFR-IgG was able to decrease TIMP-1 levels. In contrast, IL-1 $\beta$ did not induce TIMP-1 levels and subsequent addition of TNFR-IgG had no effect. TIMP-1 protein levels were further evaluated in an organ culture model of CD and UC patients (Louis et al, 2000). The production of TIMP-1 was either undetectable or below the sensitivity level in the vast majority of uninflamed samples either from controls or from patients with CD or UC. In inflamed mucosa, the production of TIMP-1 increased significantly in both CD and UC patients.

TIMP-1 and -2 expression was further investigated in myofibroblast cultures established from fibrotic CD mucosal samples (McKaig et al, 2003). A significantly higher concentration of TIMP-1 was found in myofibroblasts isolated from fibrotic CD patients compared with similar 
cells isolated from normal or UC tissue. No differences in TIMP-2 levels were observed in myofibroblasts isolated from patients with $\mathrm{CD}$, UC or control individuals. Furthermore, T cell derived IL-21 stimulation did not enhance protein expression of TIMP-1 and -2 in intestinal fibroblasts isolated from CD mucosal biopsies (Monteleone et al, 2006). The expression of TIMP-1 and -2 was also evaluated in intestinal explants from IBD patient mucosal biopsies (Meijer et al, 2007a). The expression of TIMP-1 was increased in IBD versus control explants and the highest levels were found in inflamed mucosa compared with non-inflamed and control mucosa. TIMP-2 levels were not altered between IBD and control explants. No significant differences in TIMP levels were observed between UC and CD patients. Importantly, in this study also MMP levels were measured and the authors found that there was an increased net activity of MMPs due to an imbalance between MMP and TIMP levels. Furthermore, TIMP-1 protein production in gut plasma cells from patients with IBD did not differ from controls (Gordon et al, 2008) and no effect of thalidomide, immunomodulatory drugs CC-5013 and CC4047, or PDE4 inhibitor CC-10004 was found on TIMP-1 expression (Gordon et al, 2009).

\section{Cellular localization studies}

The cellular localization of TIMP protein expression is symbolized in Figure 4. Cellular expression of TIMP-1 was found in activated fibroblasts and vascular smooth muscle cells in inflamed UC and CD tissue at the ulcer base (Arihiro et al, 2001). In biopsy specimens from patients with UC, TIMP-1 and -2 were found to be expressed in stromal cells, but not in epithelial cells (Matsuno et al, 2003). Moreover, the expression of MMP-7 was increased compared with that of the TIMP-1 and -2 , resulting in a disturbed balance. The expression of TIMP-1, -2, and -3 was further studied in resected fistula specimens from CD patients by (Kirkegaard et al, 2004). In normal tissue, TIMP-1 protein expression was only observed in a few mononuclear cells in the lamina propria, whereas TIMP-2 and -3 were observed in colonic epithelium and in a few lamina propria mononuclear cells. 
The expression of TIMP-1, -2 and -3 was further investigated by Makitalo and colleagues in three different studies. First, the expression of TIMP-1 and -3 was studied following immunosuppressive treatment in CD tissues (Makitalo et al, 2009). Stromal TIMP-1 and -3 decreased after treatment, whereas expression of epithelial TIMP-3 by enterocytes tended to increase. TIMP-1 expression by macrophages correlated with the total histological score and calprotectin levels followed a similar pattern as stromal TIMP-1, and -3. Moreover, macrophage TIMP-1 and stromal TIMP-3 positively correlated with Crohn's disease endoscopic index of severity (CDEIS). In contrast, epithelial TIMP-3 had a negative correlation with CDEIS and CRP. In a second study in pediatric IBD patients, stromal TIMP-3 expression was higher in mucosal tissues from both UC and CD patients compared with non-IBD samples (Makitalo et al, 2010). Moreover, no differences in mucosal TIMP-3 expression were observed between UC and CD patients. Increased stromal TIMP-3 expression could therefore be used as histological indicator of IBD disease activity. Finally, TIMP-1, -2 and -3 levels were investigated in pediatric UC patients with pouchitis (Makitalo et al, 2012a). In most pouch samples, the expression of epithelial and stromal TIMP-1 and -2 was lacking. Moreover, TIMPs did not correlate with inflammatory markers of inflammation (fecal calprotectin, ESR and CRP).

In our study, we observed the presence of TIMP-1 in enteroendocrine cells in the crypts. However, we did not observe differences in cellular localization or signal strength between inflamed and control tissues (de Bruyn et al, 2014b).

\section{Quantification studies}

No significant changes in TIMP-1 protein levels were observed in inflamed tissue from pediatric UC and CD patients versus normal tissue (Heuschkel et al, 2000). Moreover, no differences in TIMP-1 levels were observed between healthy controls and IBD patients, whereas patients with colon cancer had significantly elevated TIMP-1 levels (Holten-Andersen et al, 2002). However, these data were later not reproduced in other studies. In a study by 
Wiercinska-Drapalo and colleagues, plasma concentrations of TIMP-1 were found to be significantly elevated in UC patients compared with healthy controls (Wiercinska-Drapalo et al, 2003). Moreover, TIMP-1 levels positively correlated with endoscopic degree of mucosal injury, disease activity index, clinical activity index and CRP levels. In line with these data, Rath et al. found that TIMP-1 levels were significantly increased in inflamed UC tissues compared with healthy tissues, whereas TIMP-1 levels did not differ between healthy mucosa and inflamed mucosa of CD patients (Rath et al, 2006). Furthermore, the levels of TIMP-1, -2 and -4 were measured in serum of UC and CD patients (Kapsoritakis et al, 2008). Both UC and CD patients had higher serum TIMP-1 levels compared with healthy controls and the levels were higher in IBD patients with active disease compared with inactive disease. Interestingly, male patients had significantly higher mean serum TIMP-1 levels compared with females. TIMP-2 serum levels did not differ between UC and CD patients or healthy controls. TIMP-4 levels were lower in UC and CD patients compared with healthy controls and were found to be significantly lower in males compared with females. Moreover, TIMP-1 and TIMP-4 levels significantly correlated with CRP and serum amyloid A levels. Finally, the effect of immunosuppressive treatment on TIMP levels was investigated in the serum of pediatric IBD patients (Makitalo et al, 2012b). TIMP-1 and the ratio of MMP-7/TIMP-2 were found to be decreased after treatment with glucocorticoids. 


\section{ANIMAL MODEL STUDIES OF MMPS AND TIMPS}

\subsection{Association studies}

\section{Involvement of MMPs and TIMPs in animal models of colitis}

To study the function of MMPs and TIMPs in biological processes, animal model studies are required whereby experimental settings can be controlled or manipulated. Although the complex spectrum of human IBD phenotypes is not (yet) reproduced in any animal model, with the use of animals it is possible to dissect the roles played by single molecules or single cell types (Jones-Hall \& Grisham, 2014). In addition, genetic heterogeneity, as observed for HLA/MHC genes and more than 200 other genes in humans, can be excluded by using control and IBD mice with the same genetic background. Another advantage of animal model systems is the control of environmental factors, including food and drinking and eventually the (gut) microbiomes. These elements, pointing to much more simplified conditions in animals versus humans, need to be considered when comparing animal model studies with studies in patient cohorts.

In several animal association studies the involvement of MMPs and TIMPs in experimental colitis has been investigated (Table 7). In a study by Tarlton et al., experimental colitis was induced by transfer of non-fractionated CD4+ splenic T cells to immunodeficient SCID mice (Tarlton et al, 2000). The authors investigated the role of serine proteinases and MMPs in this model of colitis by zymography and by inhibition studies. Pancreatic trypsin and neutrophil elastase as well as MMP-9 were upregulated in colitis. Moreover, activated forms of MMP-2 and MMP-9 were found in colonic extracts. Transmural inflammation was associated with serine proteinase and MMP activity in epithelium and subepithelial stroma. In DSS and TNBS models of colitis, macrophage migration inhibitory factor (MIF) antibodies were investigated as well as the influence of these antibodies on MMP-13 expression (Ohkawara et al, 2002). Clinical signs and histopathological parameters of colitis were significantly attenuated after 
anti-MIF antibody administration in DSS- and TNBS-treated mice. Expression of colonic TNF$\alpha$ and IFN- $\gamma$ was reduced, whereas IL-4 expression was increased by the anti-MIF antibody. Inhibition of MIF also prevented upregulation of MMP-13 after DSS administration. Therefore, the authors stated that MIF potentially causes tissue damage via upregulation of inflammatory cytokines such as TNF- $\alpha$ as well as MMPs. The involvement of MMP-2 and MMP-8 in DSSinduced colitis was determined with immunohistochemical analyses (Pirila et al, 2003). MMP8 expression was found in colonic surface epithelial cells and was abundantly present in inflamed colon. MMP-2 co-localized with laminin-5 gamma2-chain in colonic surface epithelial cells and in the basement membrane. In inflamed colon, MMP-2 localized in epithelial cells in the lower parts of the crypt and surrounding degraded crypts.

Claudin-1 is a tight junction protein and MMP substrate (Cauwe, Van den Steen, Opdenakker, 2007) and its expression has been shown to be increased in areas of active inflammation in IBD (Weber et al, 2008). To investigate the role of claudin-1 in regulation of epithelial homeostasis and mucosal inflammation in IBD, colitis was induced in villin-claudin-1 transgenic mice (Pope et al, 2014). Colonic claudin-1 overexpression resulted in increased MMP-9 expression and Notch- and ERK1/2-signaling. In addition, the number of goblet cells and MUC-2 expression were decreased. After induction of colitis via DSS administration, villin-claudin-1 transgenic mice had an impaired recovery as compared with WT mice. In conclusion, increased claudin-1 expression leads to induction of MMP-9 and p-ERK signaling and activates Notch-signaling, which in turn inhibits goblet cell differentiation. Decreased amounts of goblet cells result in decreased MUC-2 expression and thereby an increased susceptibility to mucosal inflammation. In a study by Kundu et al., it was investigated whether Salmonella enterica serovar typhimurium regulates host PPAR $\gamma$ during infectious colitis (Kundu et al, 2014). Moreover, the involvement of PPAR $\gamma$ in the etiology of colitis was investigated through induction of colitis in KO mice with selective epithelial PPAR $\gamma$ ablation. Mice infected with S. typhimurium had decreased 
PPAR $\gamma$ expression in the intestinal epithelium and increased expression of NGAL/lipocalin-2 (Lcn2). Selective deletion of PPAR $\gamma$ in intestinal epithelial cells resulted in more severe colitis after challenge with $S$. Typhimurium, resulting in an elevated immune response (TNF- $\alpha$, IL-6, IL-17 and IL-22) and increased Lcn2 expression. Furthermore, increased levels of Lcn2 were shown to stabilize proMMP-9 via direct protein-protein interactions and this aggravated the development of colitis. Since previous studies have shown that S. Typhimurium is resistant to Lcn-2 anti-microbial activity, Lcn2 KO mice were infected with S. Typhimurium to investigate the specific role of Lcn2. Lcn2 KO mice displayed lower amounts of proMMP-9 expression compared to PPAR $\gamma$ KO mice and showed protection against colitis. These data suggest a reduced extracellular stability and possible degradation of MMP-9 protein in the absence of Lcn2. This novel mechanism by which S. Typhimurium exploits Lcn2 and MMP-9 complex interaction to induce more severe inflammation and colitis might lead to new therapeutic intervention strategies to combat salmonellosis.

TACE is able to cleave membrane-bound TNF- $\alpha$ to bioactive soluble TNF- $\alpha$ and its involvement in IBD has been previously described (Freour et al, 2009). TIMP-3 is the endogenous inhibitor of TACE and is positively associated with silent information regulator (SIRT)-1. After induction of colitis via DSS administration, the expression of TACE was increased, whereas TIMP-3 and SIRT-1 mRNA levels were decreased (Sharma et al, 2014). Treatment with a SIRT-1 activator (resveratrol) resulted in increased expression of SIRT-1 and TIMP-3, whereas TACE mRNA expression was suppressed. These effects were associated with amelioration of colitis. Treatment with TACE inhibitor also resulted in amelioration of DSSinduced colitis. This study highlights the involvement of TACE in colonic inflammation and indicates that inhibition of TACE directly or indirectly via SIRT-1 is able to ameliorate colitis. In a DSS-induced rat model of colitis, a positive correlation of disease activity index with the degree of histopathological damage and increased levels of MMP-9 and myeloperoxidase/MPO 
was shown (Oliveira et al, 2014). In previous studies it was shown that homocysteine levels were increased in plasma and mucosal tissue from CD patients (Kallel et al, 2011). The effect of homocysteine on intestinal permeability was investigated in a TNBS-induced model of colitis in rats (Ding et al, 2014). The severity of inflammation, MPO activity, levels of IL-1 $\beta$, IL-6, TNF- $\alpha$, MMP-2, MMP-9 in the colon and intestinal permeability in small intestine were significantly increased after homocysteine injection in rats with TNBS-induced colitis. These data suggest that homocysteine aggravates TNBS-induced colitis through an increase in MMP2 and MMP-9 expression which leads to disruption of the intestinal barrier.

\section{Involvement of MMPs and TIMPs in animal models of intestinal fibrosis.}

In a model of TNBS-induced chronic inflammation and fibrosis, increased expression of both MMP-1 and TIMP-1 has been described (Lawrance et al, 2003). Isolation of mesenchymal cells from TNBS-treated mice showed that these are morphologically distinct compared to mesenchymal cells isolated from control mice. Moreover, these cells expressed increased levels of TIMP-1 after IFN- $\gamma$ treatment. Transforming growth factor $\beta$ (TGF- $\beta$ ) is known to play a key role in intestinal fibrosis and its signal transduction is mediated through TGF- $\beta$ receptors, including the TGF- $\beta$ type 1 receptor also known as activin receptor-like kinase 5 (ALK5). In two animal models of fibrosis (anaerobic bacteria and TNBS), the effect of disruption of the TGF- $\beta$ /ALK5/Smad pathway was investigated by inhibition of ALK5 with SD-208 (Medina et al, 2011). Increased levels of ALK5 and TIMP-1, phosphorylation of Smad2 and Smad3 proteins and increased collagen deposition in the intestinal wall were observed in both experimental animal models. Administration of SD-208 reduced these effects. The authors concluded that ALK5 and Smad proteins can mediate TIMP-1 and collagen synthesis and these data show promise for therapeutic intervention of the TGF- $\beta /$ ALK5/Smad pathway in intestinal fibrosis. 
The effects of homocysteine on the development of intestinal fibrosis were investigated in TNBS-induced rat model (Ding et al, 2015). Administration of homocysteine to rats with TNBS-induced colitis was found to aggravate the severity of intestinal inflammation with higher levels of TNF- $\alpha$, IL-1 $\beta$ and IL-6. Moreover, levels of collagen type I and III increased in animals with intestinal fibrosis treated with homocysteine. Finally, increased levels of TGF$\beta 1$, MMP-2, MMP-9 and CTGF and mRNA expression of TGF- $\beta 1, M M P-2, M M P-9$ and TIMP1 were observed in the colon after combined TNBS and homocysteine administration. In conclusion, the authors showed that homocysteine promotes intestinal fibrosis in rats with TNBS/ethanol-induced colitis and thereby promotes expression of profibrogenic cytokines and influences the balance between MMPs and TIMPs.

\section{Effect of therapeutic intervention on MMPs and TIMPs in experimental colitis models.}

In addition to specific synthetic MMP inhibitors (vide infra \& Table 9), some natural products or therapeutic interventions have been described to influence MMP expression and thereby ameliorate intestinal inflammation. Some of these have also previously been summarized by O’Sullivan and colleagues (O'Sullivan et al, 2015).

Administration of Fumigaclavine $\mathrm{C}$, a fungal metabolite, to TNBS-treated mice significantly reduced weight loss and mortality rate (Wu et al, 2005). It reduced the expression of MMP-9 in sacral lymph node cells, colonic patch lymphocytes and inflamed tissues. Moreover, it caused a dose-dependent reduction in MMP-9 activity. Auraptene is a natural product which occurs in a variety of citrus fruits and was previously found to suppress MMP-7 expression in HT-29 cells (Kawabata et al, 2006b). Administration of auraptene to mice with DSS-induced colitis resulted in suppression of MMP-7 as well as MMP-2 and -9 expression, although the exact effect on severity of colitis needs to be further investigated (Kawabata et al, 2006a). The effect of nanocrystalline silver (NPI 32101) and sulfasalazine on the development of colitis was evaluated in a rat DNBS model (Bhol \& Schechter, 2007). Both compounds significantly 
reduced colonic inflammation and the expression of MMP-9 as well as MMP-9 activity. In a study by Ishida et al. it was investigated whether chymase, a chymotrypsin-like serine protease, contributes to MMP-9 activation in colonic tissue from DSS-treated mice (Ishida et al, 2008). Moreover, these authors studied the effect of chymase inhibition on the development of colitis. MMP-9 activity was found to be significantly increased in colonic extracts from DSS-treated mice. Administration of a chymase inhibitor (NK3201) resulted in significant reduction of shortening of colon length and decreases of disease activity index and histological damage score after DSS-induced colitis. Moreover, NK3201 decreased infiltrated neutrophil numbers and attenuated both chymase and MMP-9 activities. These data suggest that chymase plays an important role in the development of colitis via MMP-9 activation. In previous studies it has been shown that melatonin has an important anti-inflammatory effect in different experimental models including colitis (Cuzzocrea et al, 2001). Therefore, Esposito and colleagues investigated whether melatonin exerts a direct effect on gelatinase A and B levels and thereby influences the development of experimental DNBS-induced colitis in rats (Esposito et al, 2008). The authors found that melatonin could prevent colonic injury 4 days after DNBS-induced colitis. Melatonin administration reduced the increased proMMP-9 and MMP-2 levels after DNBS-induced colitis and this was associated with reduced expression of TNF- $\alpha$.

Growing evidence exists that phenylpropanoid glycosides (PPGs) are powerful anti-oxidants and possess multiple beneficial effects such as anti-inflammatory activity (Lee et al, 2005a; Middleton, 1988). Therefore, the effect of teupolioside, a phenylpropanoid glycoside of Ajuga reptans, was investigated in DNBS-induced colitis in rats (Di Paola et al, 2009). Oral administration of teupolioside resulted in significant reduction of diarrhea and weight loss, amelioration of colonic architecture disruption and reduction of myeloperoxidase activity and malondialdehyde levels. Moreover, teupolioside reduced proinflammatory cytokine release as well as proMMP-9 and -2 levels. Methyl donor deficiency (folate and/or vitamin B12 
deficiency) leads to increased levels of homocysteine in tissues and might promote mucosal inflammation by activating a wide range of pathways related with inflammation, cellular and oxidative stress and apoptosis (Peyrin-Biroulet et al, 2007). Increased levels of homocysteine have been found in plasma and colonic mucosa of IBD patients (Danese et al, 2005; Morgenstern et al, 2003). Therefore, the effect of a methyl-deficient diet (MDD; diet deprived of folate, vitamin B12 and choline) was studied in a DSS-induced colitis model in rats (Chen et al, 2011). DSS-treated rats on the MDD diet had a significantly higher disease activity index compared to DSS-treated mice on a standard diet. TIMP-3 expression was decreased in mice on the MDD diet both in control and DSS-treated mice, whereas the mRNA level of TNF- $\alpha$ was increased. In contrast, TACE levels were found to be similar in control and DSS-treated animals.

Oleuropein is the major secoiridoid in olive tree leaves and possesses a wide range of health promoting properties e.g. anti-inflammatory activities. The effect of oleuropein on DSSinduced experimental colitis in mice was investigated in order to provide insight into its mechanisms of action (Giner et al, 2011). Oral administration of oleuropein resulted in attenuation of the extent and severity of acute colitis. This was mediated through reduced neutrophil infiltration and expression of MMP-9; production of proinflammatory cytokines and the translocation of the NF- $\kappa \mathrm{B}$ p65 subunit to the nucleus in colon tissue. Since microbiota play an important role in the pathogenesis of IBD, the effect of antibiotic (minocycline) and probiotic (Escherichia coli Nissle 1917 [EcN]) treatment as well as the combination of both treatments was investigated in a mouse model of reactivated DSS-induced colitis (Garrido-Mesa et al, 2011). Both minocycline and $\mathrm{EcN}$ had anti-inflammatory effects and were able to attenuate the reactivation of colitis. The combination of the two treatments resulted in a greater effect than the individual treatments. A reduced expression of TNF- $\alpha$, IL-1 $\beta$, IL-2, MIP-2, MCP-1, ICAM1, iNOS and MMP-9 was observed as well as an increased expression of MUC-3 and ZO-1. 
Moreover, the altered microbial composition of DSS-treated mice was partially restored. These data indicate that the combination of antibiotic and probiotic treatments improves colonic inflammation in mice and thus support the potential use of this association in controlling intestinal inflammation.

Pathologic angiogenesis and uncontrolled expression of angiogenic growth factors have been linked with development and progression of IBD (Scaldaferri et al, 2009; Tolstanova et al, 2009). Endostatin is an anti-angiogenic factor which is cleaved from collagen XVIII by MMP9 (Nilsson \& Dabrosin, 2006). The involvement of endostatin in IBD was evaluated with three different experimental colitis models: 1) $6 \%$ iodoacetamide (IA) in rats, 2) 3\% DSS in MMP-9 KO and WT mice and 3) IL-10 KO mice (Tolstanova et al, 2011). In all three models, levels of VEGF, PDGF, MMP-9 and endostatin were upregulated in diseased mice. In MMP-9 KO mice, the levels of endostatin were decreased in both control and DSS-treated mice. Moreover, treatment with endostatin significantly improved DSS-induced colitis in MMP-9 KO mice. These data show that MMP-9 is a key enzyme for generation of endostatin and anti-angiogenic treatment may be considered as a new therapeutic option in IBD. Vasoactive intestinal peptide (VIP) is expressed in primary immune organs and lymphoid follicles of many tissues and is a potent neuroendocrine mediator of diverse physiological responses. The roles of two $\mathrm{T}$ cell $\mathrm{G}$ protein-coupled receptors (VPAC1 and VPAC2) in regulation of immune homeostasis and immuno-pathological reactions were investigated in a DSS-induced murine acute colitis model (Yadav et al, 2011). VPAC-2 KO mice showed increased susceptibility to DSS-induced colitis with enhanced infiltration of neutrophils and increased expression of MMP-9. In contrast, VPAC-1 KO mice were found to be resistant to DSS-induced colitis. These data suggest that VPAC-1 plays an enhancing role in the development of colitis and may be considered as a therapeutic target. 
The effect of Etiasa, which belongs to the aminosalicylic acid class of drugs, was investigated in a rat model of TNBS-induced colitis (Mao et al, 2012b). The authors showed that Etiasa was able to reduce the increased expression of MMP-2 and TNF- $\alpha$ in the diseased rats. The therapeutic effect of irsogladine maleate (IM), widely used as an anti-ulcer drug for gastric mucosa in Japan, was investigated in a DSS-induced murine model of chronic fibrostenosing colitis (Yamaguchi et al, 2012). In this model, increased levels of MMP-2 and TIMP-1 were observed. After IM administration, clinical and histopathological parameters of chronic DSSinduced colitis were attenuated. Moreover, collagen deposition was diminished and MMP-2 and TIMP-1 expression was significantly decreased. $\alpha$-Lipoic acid (LA) is a naturally occurring sulfur-containing fatty acid and is a potent anti-oxidant and anti-inflammatory agent (Odabasoglu et al, 2011). Administration of LA to mice with chronic DSS-induced colitis resulted in amelioration of clinical and inflammatory parameters as well as decrease of MMP9 levels (Trivedi \& Jena, 2013). Cordyceps militaris (CM) extracts have been shown to exert strong anti-inflammatory and anticancer activities (Cho et al, 2007) and are widely used among traditional medicines in East Asia to treat inflammatory diseases. Since CM is difficult to obtain in large amounts due to the high unit cost of production, germinated soybeans were used as culture media for CM (Park \& Park, 2013). Germinated soybean CM was able to reduce disease activity index and histopathological parameters of DSS-induced colitis. Moreover, it reduced MMP-3 and -9 mRNA expression levels.

Due the fact that at present not one single treatment option is available to cure IBD patients, unconventional strategies are currently under investigation. One of these is the use of mesenchymal stem cells (MSCs). MSCs have emerged as a promising therapeutic candidate for diseases that are caused by abnormal immune responses due to their anti-inflammatory and reparative properties. In a recent study by Banerjee et al., the protective potential of human umbilical cord mesenchymal stem cells (UCMSCs) was investigated in an experimental model 
of DSS-induced colitis in immunodeficient NOD.CB ${ }_{17^{-}}{ }^{\text {Prkdcscid }} / \mathrm{J}$ mice (Banerjee et al, 2015). The administration of UCMSCs was shown to significantly ameliorate DSS-induced colitis. This effect was accompanied by a decrease in neutrophil infiltration, MPO levels, MMP-2 and MMP-9 protein levels as well as a positive modulation in ER stress related proteins in DSStreated mice injected with UCMSCs.

In conclusion, various unconventional approaches, including nutrient, probiotic, antibiotic, pharmacological and cell therapies, have been used as tests for treating animals with IBD-like syndromes. In most cases beneficial effects on disease scores correlated with a restoration of an imbalance between MMPs and TIMPs levels. 


\subsection{Gene knockout and transgenic studies}

Several mouse strains deficient of MMPs and TIMPs have been engineered and discussed elsewhere (Gill et al, 2010). Some of these MMP or TIMP deficient mice have been used for studies on their pivotal role in intestinal inflammation (Table 8).

\section{MMP-2}

MMP-2 (or gelatinase A) is expressed in a constitutive manner (Van den Steen et al, 2002; Vandooren et al, 2013b). However, MMP-2 levels have been shown to be upregulated during active flares in IBD patients and in experimental animal models of colitis. To investigate its pivotal role in IBD, colitis was induced with the use of two different models (DSS and Salmonella enterica subsp. serovar Typhimurium (S.T.)) in WT and MMP-2 KO mice (Garg et al, 2006). In WT mice, MMP-2 protein and activity levels were found to be increased after both DSS and S.T. administration Moreover, MMP-2 KO mice had aggravated development of colitis induced by both DSS and S.T. compared with WT mice. MMP-2 expression was found to be increased in both epithelial and infiltrating immune cells. In addition, bone marrow chimeras were performed with the use of bone marrow cells from WT and MMP-2 KO mice. These experiments showed that mucosa-derived MMP-2 is required for its protective effect during intestinal inflammation. Interestingly, the aggravated colitis observed in MMP-2 KO mice was found to be independent of compensation by MMP-9. Finally, in vivo permeability tests indicated that MMP-2 KO mice had an increased intestinal permeability compared to WT mice. MMP-2 is thus an important player to maintain epithelial barrier function. A few years later, these data were contradicted in a study by Heimesaat et al. (Heimesaat et al, 2011b). The authors investigated development of DSS-induced colitis in MMP-2 KO mice and found that these mice were protected from development of colitis. MMP-2 KO mice showed less body weight loss, lowered clinical colitis score, longer colon lengths, lower histopathological score and less pronounced immune cell response after DSS administration compared to WT mice. 
Moreover, MMP-2 KO mice had lowered colonic E. coli loads compared to WT mice. These data therefore suggest that MMP-2 plays an important disease-causing role instead of a diseaseprotecting role. The authors speculate on the reason of discrepancy with the previous study by Garg et al., pointing towards the important role of age, sex, origin and genetic background of the used mice as well as percentage of DSS and duration of colitis. Moreover, the role of the microbiota composition in initiation, mediation and perpetuation of colitis is currently the topic of many studies and is influenced by several factors (e.g. animal facility, diet, genetic background, infection status). Also this confounding factor may have played a role in such different outcomes of both studies. Recently, the role of MMP-2 in the development of infectious colitis was further investigated (Alutis et al, 2015b). In this study, infant MMP-2 KO mice were per orally infected with Campylobacter $(C$.) jejuni strain B2 immediately after weaning. MMP-2 KO mice showed protection against development of infectious colitis, with less blood in the feces, lowered histopathological score and apoptosis, lowered number of immune cells and elevated IL-22 mRNA levels. Moreover, in another study by Alutis et al., the role of MMP-2 in C. jejuni infection was further investigated in gnotobiotic mice (Alutis et al, 2015a). The mice were treated with broad-spectrum antibiotic treatment and then per orally infected with $C$ ) jejuni strain 81-176. After 8 and 14 days post infection, MMP-2 KO mice had lowered histopathological changes, less epithelial apoptosis, reduced numbers of immune cells and increased expression of IL-22, IL-17A and IL-1 $\beta$. However, MMP-2 KO mice showed more epithelial proliferation compared to WT mice. Data obtained by both studies point towards a disease-causing role of MMP-2 in $C$. jejuni mediated infectious colitis and are in line with the previous study by Heimesaat et al. (Heimesaat et al, 2011b).

\section{MMP-3}


In a study by Li et al., the role of MMP-3 in infectious colitis was investigated. MMP-3 KO mice were found to have similar extents of crypt hyperplasia in response to $C$. rodentium infection as WT mice (Li et al, 2004). However, MMP-3 KO mice showed delayed occurrence of $\mathrm{CD}^{+}$cell infiltrates and TNF transcripts in the infected colon compared to WT mice. Consistent with reduced infiltrate of CD4+ cells, lower amount of $\beta 7+$ cell were observed. Deletion of MMP-3 was found to be compensated by elevated expression of other MMPs, namely MMP-7 and MMP-12, as well as TIMP-1. These data suggest that MMP-3 plays a critical role in mobilization of $\mathrm{T}$ cells in the lamina propria and controlling pathogenic bacterial populations.

\section{MMP-7}

The role of MMP-7 in experimental colitis was investigated by several knock-out studies. First, Wilson et al. described that MMP-7 KO mice had more severe infectious colitis induced by S.T. compared to WT mice (Wilson et al, 1999). The authors concluded that MMP-7 is involved in host defense against bacteria through activation of defensins. In a second study, it was found that MMP-7 KO mice produce pro-defensins, but not mature defensins -due to lack of processing by MMP-7- and developed more severe DSS-induced colitis (Shi et al, 2007). Moreover, the expression of IL-1 $\beta$ was found to be increased in MMP-7 KO mice both at baseline and after induction of colitis with DSS. In addition, the link between defensin deficiency and increased IL-1 $\beta$ production was further elucidated by addition of defensins to LPS-activated mouse peritoneal macrophages. In these experiments it was clear that only mature defensins are able to inhibit IL-1 $\beta$ release from LPS-activated monocytes. In contrast, no effect of defensins was found on the secretion of TNF- $\alpha$ by LPS-activated monocytes. Third, Swee and colleagues found a dual role for MMP-7 in the development of colitis (Swee et al, 2008). A lower mortality rate was found for MMP-7 KO mice after DSS administration compared to WT mice. However, re-epithelialization appeared to be delayed in MMP-7 KO 
mice and the overall observed intestinal injury was not significantly different from WT mice. The difference in mortality rate was found to be due to differences in location and extent of infiltrated neutrophils in the gut, which was related to chemokine expression. MMP-7 is therefore thought to control neutrophil movement by generating a transmucosal chemokine gradient. Finally, Vandenbroucke et al. described that MMP-7 KO mice were protected against LPS-induced intestinal permeability and inflammation (Vandenbroucke et al, 2014). They found that $\alpha$-defensins are able to stimulate macrophages to secrete IL-6 in a TLR-4 independent way. Besides previously described bactericidal and anti-inflammatory roles, the activation of $\alpha$-defensins by MMP-7 is therefore considered to play a pro-inflammatory role through activation of macrophages and amplification of the local inflammatory response.

\section{MMP-9}

Several authors investigated the role of MMP-9 (or gelatinase B) in experimental colitis. MMP9 is an inducible protease which has been shown to be upregulated in several inflammatory conditions (Opdenakker et al, 2001b). In a study by Castaneda et al., the effect of MMP-9 deletion on the development of DSS- and S. typhimurium-induced colitis was investigated (Castaneda et al, 2005). MMP-9 KO mice showed attenuated development of DSS-induced colitis with less body weight loss, less diarrhea and fecal occult blood, lowered clinical colitis score and lowered histopathological score. Moreover, MMP-9 KO mice were also protected from oral S. typhimurium-induced colitis. The authors further investigated whether neutrophilderived MMP-9 is required for migration of neutrophils to the site of inflammation. However, no difference in transepithelial migration was found between neutrophils derived from WT or MMP-9 KO mice. In addition, bone marrow transplantation experiments showed that WT mice that received bone marrow from MMP-9 KO mice developed aggravated colitis that manifested earlier compared to WT mice that received WT bone marrow. Moreover, MMP-9 KO mice that received WT bone marrow had increased neutrophil infiltration compared to MMP-9 KO mice 
that received MMP-9 KO bone marrow. However, no difference in clinical and histopathological parameters of colitis was found between these mice. Therefore it was concluded that not neutrophil-derived MMP-9, but mucosal (epithelial) MMP-9 is required for tissue damage in DSS-induced colitis. The attenuation of colitis in MMP-9 KO mice was also observed in a study by Santana et al. (Santana et al, 2006). The authors observed significantly lowered body weight loss, higher survival rates and attenuated extent and severity of intestinal injury in MMP-9 KO mice compared to WT mice. Interestingly, DSS-induced colitis parameters correlated well with pro-MMP-9 levels, but not with MMP-2 levels. In contrast, MMP-2 levels were upregulated during the recovery phase of colitis, whereas pro-MMP-9 levels decreased in this phase. These data suggested that MMP-9 is mainly involved in mucosal injury whereas MMP-2 seems to be linked with mucosal repair. However, previously mentioned MMP-2 KO studies are not able to confirm this hypothesis due to inconsistent results.

Since epithelial MMP-9 is considered the critical mediator of tissue damage and MMP-9 KO mice are protected from colitis, Garg et al. further studied the role and mechanism of MMP-9 in epithelial barrier function (Garg et al, 2007). They found that MMP-9 KO had increased number of goblet cells and MUC-2 expression. In addition, transcription factors involved in terminal differentiation of goblet cells, KLF-4 and Elf-3, were found to be upregulated, whereas activated Notch-1 was downregulated in MMP-9 KO mice. Interestingly, these data can be linked to a previously discussed study by Pope et al. (Pope et al, 2014), whereby claudin-1 overexpression led to induction of MMP-9, p-ERK- and Notch- signaling and inhibition of goblet cell differentiation and MUC-2 expression. Although cancer is not the topic of this review, it was shown that MMP-9 KO mice have an increased susceptibility to colitis-associated cancer (Garg et al, 2010). MMP-9 is thus considered as an amplifier of intestinal inflammation, but a suppressor of colonic tumor formation. 
In contrast to previously discussed studies in which MMP-9 KO mice were shown to have attenuated development of colitis, Heimesaat et al. found no difference in the development of colitis between WT and MMP-9 KO mice (Heimesaat et al, 2011b). In addition, our group reinvestigated the role of MMP-9 by inducing acute and chronic IBD with DSS in an optimally controlled colony of $\mathrm{KO}$ and wild-type mice and we found no differences (PhD Thesis Magali de Bruyn; KU Leuven, 2016 \& manuscript in preparation). In our studies, we used littermate wild-type and KO mice with the same genetic background (13 backcrossed into C57BL/6J), reared for more than 15 years in the same SPF insulator with exactly the same environmental conditions, including food and beverages and with similar microbiomes. As mentioned previously (Vanden Berghe et al, 2015), differences in age, sex and genetic background of the mice as well as differences in the used $\mathrm{KO}$ and colitis model probably are the causes behind opposite observations between studies. As previously documented (Hu et al, 2007), a strong need exists for better controlled KO studies with better genotyping and environmental and microbiome matching of wild-type and KO mice.

Indeed, substantial differences in microbial composition could be the cause of the observed discrepancies. Therefore the authors propose that future studies should perform a detailed survey of the gut microbiota in order to allow comparison of data obtained in different animal facilities. In another study, in which the important role of the microbiota was emphasized, also no significant differences were found in development of colitis in MMP-9 KO versus WT mice (Rodrigues et al, 2012). In this study, an infectious colitis model was used whereby $C$. rodentium was orally administered to WT and MMP-9 KO mice. The response to infection, colonic epithelial cell hyperplasia and barrier dysfunction were similar in WT and MMP-9 KO mice. Moreover, the authors performed nonmetric multidimensional scaling of terminal restriction fragments which indicated a different fecal microbiome composition and $C$. rodentium colonization pattern in WT and MMP-9 KO mice. MMP-9 KO mice had lower levels 
of $C$. rodentium and elevated levels of protective segmented filamentous bacteria and interleukin-17. Finally, MMP-9 KO mice were found to be protected against reductions in microbial diversity in response to infection. These data indicate a role for MMP-9 in disturbance of the microbial composition during bacterial-induced colitis. In a study by Alutis et al., it was shown that infant MMP-9 KO mice responded to $C$. jejuni infection in a similar way as infant WT mice. However, significantly lowered amounts of colonic neutrophils and T cells were seen in MMP-9 KO mice as compared to WT mice post-infection. Moreover, in a follow-up study by Alutis et al., whereby adult mice were first treated with broad spectrum antibiotics and then infected with $C$. jejuni, it was confirmed that MMP-9 KO mice were not protected from infectious colitis (in contrast to MMP-2 KO mice) (Alutis et al, 2015a). In addition, the observation of a less pronounced influx of immune cells in the colon was also confirmed in this study. In a recent study by Nighot et al., the role of MMP-9 in intestinal permeability was further investigated (Nighot et al, 2015). The severity of colitis and increased intestinal permeability after DSS administration were found to be attenuated in MMP-9 KO mice compared to WT mice. The tight junctional occludin level was found to be increased after DSS compared with control tissue in MMP-9 KO mice. Moreover, previous studies have shown that intestinal barrier function is regulated by myosin light chain kinase (MLCK) via MLC phosphorylation (Shen et al, 2006). MMP-9 KO mice were found to have lowered expression of MLCK and phospho-MLC. These data indicated that MMP-9 modulates both intestinal permeability and severity of inflammation through increased MLCK expression.

Next, the effect of double KO of both gelatinases (MMP-2 and MMP-9) on the development of colitis was investigated (Garg et al, 2009). MMP-2/-9 double KO mice were found to be protected against development of colitis in three different models (DSS, S. typhimurium, and TNBS). These data, together with previous data by these authors that MMP-2 KO mice have worsened colitis and thus MMP-2 plays a protective role (Garg et al, 2006), suggest that tissue 
injury mediated by MMP-9 overrides the barrier protective role of MMP-2. Therefore the authors conclude that inhibition of MMP-9 as therapeutic option may be beneficial even if this also results in MMP-2 inhibition.

As another approach to KO mice studies to investigate the pivotal role of MMP-9 in intestinal inflammation, the effect of MMP-9 overexpression on the development of colitis was investigated in two independent studies. First, DSS and S. typhimurium colitis was induced in MMP-9 transgenic mice (Tg-villin-MMP-9) with targeted epithelial overexpression of MMP9. Tg-villin-MMP-9 mice had significantly decreased amounts of goblet cells, increased epithelial proliferation and apoptosis, and increased expression of proinflammatory cytokine Kc compared with WT mice. After induction of colitis, Tg-villin-MMP-9 mice showed an aggravated inflammatory response with greater weight loss, higher clinical scores, higher histopathological scores and MPO activity compared to WT mice. The authors therefore concluded that MMP-9 secreted by intestinal epithelial cells mediates inflammation with increased expression of Kc. Recently, Nighot et al. described that injection of exogenous MMP9 in healthy WT mice resulted in an increased colonic permeability in a dose-dependent manner (Nighot et al, 2015). The MMP-9-injected mice, however, did not show any symptoms of colitis, histopathological signs of inflammation in the colon or elevated proinflammatory cytokine levels. These data indicate that the MMP-9-mediated increase in colonic permeability did not result from colonic inflammation, nor did it lead to colonic inflammation. The role of MMP-9 in intestinal permeability and inflammation can therefore be considered as an important 'chicken or the egg' research question which needs to be further investigated.

\section{MMP-10}

In addition, Koller et al. studied the development of acute and chronic DSS-induced colitis in MMP-10 KO mice (Koller et al, 2012). Deletion of MMP-10 resulted in more severe development of colitis with higher disease scores and failure to resolve inflammation even after 
extended recovery periods. MMP-10 was found to be produced predominantly by infiltrating myeloid cells (mostly macrophages). Moreover, bone marrow transplantation experiments indicated that MMP-10 production by these cells reduces severity of colitis. In addition, MMP$10 \mathrm{KO}$ mice had a higher propensity for development of dysplastic lesions. In support of previous reports of a beneficial role of MMP-10 in human IBD (Salmela et al, 2004), MMP-10 is thought to be required for resolution of colonic damage and absence of MMP-10 leads to chronic inflammation and ultimately dysplasia.

\section{MMP-12}

The role of MMP-12 in intestinal inflammation was further investigated in a TNBS-induced colitis model (Pender et al, 2006). MMP-12 KO mice were shown to be protected against development of colitis as no mucosal damage or histopathological inflammation were observed. MMP-12 expression by macrophages can be increased by local immune responses and MMP12 might therefore play a significant detrimental role in intestinal inflammation.

\section{MMP-13}

Vandenbroucke et al. investigated the pivotal role of MMP-13 in epithelial barrier disruption and the development of colitis (Vandenbroucke et al, 2013). MMP-13 KO mice were found to be protected against development of acute DSS-induced colitis, as well as LPS-induced sepsis. Goblet cell depletion, ER stress, permeability and tight junction destabilization were all found to be reduced in the gut of MMP-13 KO mice compared to WT mice. The authors showed that MMP-13 is able to cleave membrane-bound TNF into soluble TNF, identifying MMP-13 as an alternative TNF sheddase, next to TACE. These data suggest that MMP-13 is a novel therapeutic target for treatment of IBD, although caution is required towards creating compounds that are specific for MMP-13 and do not target other MMPs.

\section{MMP-19}


Recently, the role of MMP-19 is intestinal inflammation was investigated (Brauer et al, 2015). MMP-19 KO mice presented with more severe DSS-induced colitis compared to WT mice. Neutrophil infiltration into the colon was found to be delayed in MMP-19 KO mice which led to persistent inflammation and delayed recovery. Reconstitution of MMP-19 KO mice with bone marrow from WT mice resolved these problems. Moreover, MMP-19 deficient macrophages showed decreased migration and intestinal permeability was found to be increased. CX3CL1 was identified as a novel substrate of MMP-19 and was linked with the impaired cellular recruitment phenotype in MMP-19 KO mice. MMP-19 was therefore characterized to play an important role in host response and innate cellular recruitment in colitis.

\section{TIMP-1 and -3}

Since TIMPs are the endogenous inhibitors of MMPs, the effect of genetic deletion of TIMPs on the development of colitis was also investigated. In a study by Monteleone and colleagues, the effect of TIMP-3 deletion on development of colitis after TNBS administration and T cell transfer was investigated (Monteleone et al, 2012). TIMP-3 inhibits TACE which cleaves membrane-bound TNF into soluble TNF. TIMP-3 levels were found to be decreased in human CD samples, whereas TACE levels were increased. TIMP-3 KO mice had increased TACE activity and soluble TNF levels and developed more severe colitis compared to WT mice. In contrast, mice that overexpressed TIMP-3 in myeloid cells under the CD68 promotor (TgCD68-TIMP-3) were found to be protected against TNBS-induced colitis. TIMP-3 is therefore considered to be a negative regulator of inflammation in the gut with a central role for TNF. Recently, we investigated the development of colitis and fibrosis in TIMP-1 KO mice (Breynaert \& de Bruyn et al, in press). We observed that TIMP-1 KO mice recovered faster from acute DSS-induced colitis based on body weight and disease activity index. However, microscopic and macroscopic parameters indicated that TIMP-1 KO mice had more severe acute colitis compared to WT mice. In contrast, chronic inflammation and fibrosis were 
attenuated in TIMP-1 KO mice after chronic DSS administration. With gelatin zymography analysis, we could not link the observed lowered development of fibrosis in TIMP-1 KO mice with increased MMP-9 levels (Figure 5, lower panel). In contrast, we observed a trend for higher proMMP-9 levels in WT mice after chronic DSS administration. In addition, in the acute model, we observed higher pro- and activated MMP-2 levels in WT mice compared to TIMP-1 KO mice. TIMP-1 is involved in fibrosis through inhibition of MMP-related ECM degradation. However, TIMP-1 has several MMP-independent functions which may cause the paradoxical effects seen in the acute model of colitis. It also needs to be stressed that the TIMP-1 gene is Xlinked in human and mice and that all mouse TIMP-1 KO studies have been done with insufficient control of genetic background and environmental confounding factors.

In conclusion and on the basis of critical evaluation of the literature, the present role of MMP2 and MMP-9 in IBD is not clear, whereas MMP-7, MMP-10 and MMP-19 seem beneficial molecules, and MMP-12 and MMP-13 seem detrimental molecules in induced IBD models. For the TIMPs, the pictures are less clear: TIMP-3 may be protective, whereas TIMP-1 may be rather disease-promoting.

\section{STUDIES WITH MMP INHIBITORS (MMPIS)}

Since the late 1970's, at least 56 different MMP inhibitors (MMPI) have been pursued as clinical candidates. The primary indications, for which these inhibitors were used, were cancer (24 MMPIs), arthritis (27 MMPIs) and cardiovascular disease (10 MMPIs). Most of these MMPIs were discontinued in their respective clinical trials due to severe dose-related sideeffects (e.g. musculoskeletal syndrome, MSS) (Coussens et al, 2002; Fingleton, 2003). Only two have been approved by the FDA, one for the indication of periodontal disease (Periostat $\left.{ }^{\circledR}\right)$ and one for the treatment of stroke (Minocycline). In addition, a handful of MMPIs remain in clinical trials (Hu et al, 2007). The study field of MMPIs after the first tribulations therefore shifted to treatment of early stage cancer and inflammatory conditions (Overall \& Lopez-Otin, 
2002). An overview of MMPI in vascular and inflammatory diseases was previously given by Hu et al. (Hu et al, 2007) and Vandenbroucke et al. (Vandenbroucke \& Libert, 2014). More relevant to the present topic we provide a brief summary of MMPIs in relation to research on IBD (Table 9).

Treatment with the broad-spectrum hydroxamate-type MMP inhibitors batimastat and marimastat (acting on most MMPs discussed above within the frame of knockout studies) resulted in amelioration of intestinal inflammation in a TNBS-induced rat model of colitis (Di Sebastiano et al, 2001; Sykes et al, 1999). The synthetic inhibitor CGS-27023-A was tested in a DSS-induced rat model of colitis. Although DSS-treated rats had a decreased extent and severity of epithelial injury after oral administration of CGS-27023-A, the inhibitor had no effect on mucosal repair (Medina et al, 2003). In a follow-up study CGS-27023-A was also investigated in TNBS-induced rat model of colitis (Medina et al, 2006a). At the used calculated doses, the administration of CGS-27023-A was able to inhibit MMP-9, but not MMP-2, and lowered MPO activity. Moreover, early (day 0-10), but not delayed (day 11-21) treatment with CGS-27023-A was able to attenuate mucosal injury. Another orally active MMP inhibitor, ONO-4817 has been shown to inhibit MMP-2, -3, -8, -9, -12 and -13, but not MMP-1 and -7 (Yamada et al, 2000). Administration of ONO-4817 to mice with DSS-induced colitis resulted in reduced inflammatory cell infiltration and mucosal cell disruption (Naito et al, 2004). Intraperitoneal administration of MMP-3 and MMP-10-specific small interfering RNAs (siRNAs) resulted in reduced transcription of the targeted MMPs and caused attenuation of DSS-induced colitis in mice. Another broad-spectrum inhibitor ilomastat was shown to protect rats from TNBS-induced ulcerative colitis through inhibition of MMP-1 (Wang \& Wang, 2008). In addition, administration of minocycline, a semi-synthetic tetracycline and inhibitor of MMPs, was able to attenuate acute and chronic DSS-induced colitis through lowered expression 
of MMP-2, -3, -9 and -13 as well as suppression of inducible nitric oxide synthase (iNOS), nitrotyrosine and proinflammatory cytokine expression (Huang et al, 2009). Similarly, minocycline was also shown to attenuate TNBS-induced colitis in mice (Huang et al, 2009). Previously, the synthetic collagen analogue (Gly-Pro-Hyp) ${ }_{10}\left[(\mathrm{GPO})_{10}\right]$, an exemplification of the rigid collagen triple helix, was identified as a relevant binding structure for proMMP-2/-9 in the extracellular matrix (Ruehl et al, 2011) and promoted enzymatic activity of proMMP-2 (Freise et al, 2012). Treatment of mice with (GPO) 10 resulted in amelioration of clinical and histopathological parameters of DSS-induced colitis, decreased secretion of IL-6 and increased concentrations of IL-10 (Heimesaat et al, 2012). Furthermore, infiltration of inflammatory cells was reduced, whereas Tregs and regenerative cell counts were increased. RO28-2653, a selective gelatinase (MMP-2/-9) inhibitor, was previously shown to ameliorate hyper-acute small intestinal inflammation in mice infected with Toxoplasma gondii (Munoz et al, 2009). Moreover, in a study by Heimesaat et al., RO28-2653 was shown to ameliorate DSS-induced colitis in mice with less severe histopathological changes, lowered pro-inflammatory cytokine expression, less influx of immune cells into the mucosa and lowered E. coli loads in the colon (Heimesaat et al, 2011a).

AE-941, a natural inhibitor of MMPs found in shark cartilage, was found to decrease MMP-2 and -9 expression as well as to ameliorate signs of colitis after administration via gastric lavage to rats with TNBS-induced colitis (Mao et al, 2012a). Sela-Passwell et al. recently described that a selective gelatinase inhibitor (SDS3), created by molecular mimicry and directed towards the activated forms of MMP-2 and MMP-9, was able to attenuate DSS-induced colitis in mice (Sela-Passwell et al, 2012). Furthermore, the effect of proline-glycine-proline (PGP) antagonists and antibodies on DSS-induced colitis was investigated by Koelink and colleagues (Koelink et al, 2014). PGP is derived from collagen by a combination of MMP-8 and/or MMP9 and prolyl endopeptidase (PE) and was shown to have chemotactic effects on neutrophils via 
CXCR2 in lung diseases (Weathington et al, 2006). MMP-8, MMP-9 and PGP levels were found to be increased in intestinal tissue from DSS-treated mice and PGP neutralization results in significant reduction of neutrophil infiltrates in the intestine. These results show that a vicious cycle exists between MMP-8/-9 mediated collagen degradation and neutrophil infiltration in IBD. Recently, Marshall and colleagues created selective anti-MMP-9 antibodies and tested these in preclinical animal models of DSS-induced colitis as well as colorectal cancer (Marshall et al, 2015). Human MMP-9 protein was created by cloning full length MMP-9 cDNA into a pSecTag2hygro (B) vector and transiently transfecting it into HEK293 cells. The human MMP9 protein was then used to immunize BALB/c mice and finally the anti-human and anti-rat MMP-9 antibody AB0041 was identified. The anti-mouse MMP-9 mAb (AB0046) was created by immunization of MMP-9 KO mice with a mouse MMP-9 protein composed of only the pro and catalytic domains. Treatment of rats with AB0041 did not result in musculoskeletal syndrome symptoms, in contrast to marimastat. Moreover, AB0041 was able to inhibit release of sTNF by MMP-9. Treatment of DSS-induced colitis in mice with AB0046 resulted in significant reduction of weight loss, diarrhea, endoscopy scores and histological scores. Moreover, AB0046 administration resulted in 55\% reduction of total MMP-9 levels in colon tissue lysates. Finally, the authors humanized AB0041 to be able to use this antibody (GS-5745) in human clinical trials. Currently, phase 2 clinical trials with GS-5745 are ongoing in both UC and CD patients (NCT02520284 and NCT02405442).

\section{EVIDENCES FOR MMP INHIBITION AS THERAPY FOR IBD IN HUMANS}

In the past and as stated above, the use of MMPI in cancer trials has failed due to serious sideeffects (MSS). The reason for failure of most MMPIs was the non-selectivity of these inhibitors. To avoid broad-spectrum non-selective inhibition and inherent side-effects caused by inhibition of off-target MMPs, recent research has switched from targeting the MMP catalytic site to alternative, less conserved sites of MMPs. Although it still remains a challenge to identify 
MMP-specific alternative regulatory sites, several technological improvements seem promising (Udi et al, 2013). One possibility is to target the haemopexin domain of MMPs, because the latter domains possess the highest sequence diversity (Piccard et al, 2007). Moreover, in the search for more selective MMPIs, antibody-based therapeutics are now being explored (Fields, 2015). Several functional blocking antibodies that selectively target MT-MMPs have been developed (Devy \& Dransfield, 2011). In our laboratory, a selective neutralizing antibody against MMP-9 (REGA-3G12) was developed (Paemen et al, 1995). It is directed against the catalytic domain, but does not target the fibronectin or the zinc-binding domains (Martens et al, 2007). Recently, antibodies selectively targeting activated forms of MMP-2 and MMP-9 with TIMP-like binding mechanisms were described (Sela-Passwell et al, 2012). These were shown to protect against the development of colitis after DSS administration in mice. Moreover, Marshall and colleagues created allosteric antagonists specific for MMP-9 which were shown to be effective against development of acute DSS-induced colitis in mice (Marshall et al, 2015) (vide supra).

In addition to the synthesis of MMP inhibitory agents, modifications of TIMPs to target more selectively specific MMPs have been described and these have been reviewed elsewhere (Nagase et al, 2008). Differences in interaction and chemistry within the interfaces of Nterminal TIMP-1 complexes provide a basis for engineering inhibitory selectivity. For example, Hamze and colleagues demonstrated that constraining specificity in the N-domain of TIMP-1 can lead to the engineering of gelatinase-selective inhibitors (Hamze et al, 2007). Furthermore, the concept of Protease-Activated Prodrugs (PAPs) (Vandooren et al, 2016) allows for therapeutic agents to be targeted to the location of inflammation and the presence of MMPs is then required for activation of the drug. In this case, MMPs can be used indirectly as therapeutic targets in IBD. 
Despite promising results from animal models of colitis and human data that link MMPs with disease severity, no clinical trials were initiated, until recently, with MMP inhibitors as treatment for IBD. Two years ago, phase 1 clinical trials with monoclonal antibodies directed against MMP-9 in IBD patients were completed (Bhandari et al, 2015) and currently phase 2 clinical trials are ongoing. Although results from phase 1 trials indicated that anti-MMP-9 treatment may be safe and efficacious in moderate-to-severe UC patients (Bhandari et al, 2015), discrepancies in preclinical animal studies with MMP-9 KO mice conducted by us and others (Alutis et al, 2015a; Heimesaat et al, 2011b; Rodrigues et al, 2012) warrant careful follow-up of these antagonists and their use in IBD patients. Moreover some recommendations are needed with views on future studies investigating MMPs as therapeutic targets in IBD. First, more studies investigating the therapeutic potential of MMPs in IBD are required with strong emphasis on spatiotemporal expression and function in different stages of the disease. In addition, animal models that are commonly used to elucidate MMP activity and to evaluate MMP inhibitors are often acute colitis models, whereas tissue remodeling, fibrosis and stricture formation are only apparent in chronic models. Furthermore, besides detrimental roles, several MMPs are important for wound healing, which suggests that therapeutic MMP inhibition may have adverse effects. Of importance is the fact that MMPs are involved in a web of interconnected proteases (Kruger et al, 2010) and therefore disruption or therapeutic inhibition of one MMP might impact functional properties of other MMPs. This might translate into disrupted substrate cleavage and physiological processes. Therefore, multidimensional approaches are needed to unravel the pivotal roles of MMPs in intestinal inflammation including genomic and proteomic profiling techniques in addition to gene deletion and transgenic mouse models (Morrison et al, 2009). Our micro-array data (de Bruyn et al, 2014b) and the present systematic review illustrate that MMP inhibition for IBD therapy is a real challenge. If we want to keep medical research safe, the discovery of a key MMP within the 
protease network of IBD will require a lot more preclinical studies than those that are presently done.

Finally, several suggestions for further investigations with regard to the use of MMPs as therapeutic targets may come from cancer research whereby several MMPs have been defined as anti-targets (Dufour \& Overall, 2013). An elevated expression of a gene transcript or product does not always imply this particular gene can be considered as a target for the studied disease. This has been shown previously for some MMPs in cancer research whereby MMP-8, -10 and -12 were considered as anti-targets (Overall \& Kleifeld, 2006). In IBD, it appears that MMP-2, $-7,-9,-10$ and -19 can be considered as anti-targets, whereas MMP-12 and -13 may still be considered as targets based on transgenic and $\mathrm{KO}$ animal studies (see Table 8). In the case of MMP-2, -7 and -9; several animal studies indicate contradicting results with regard to diseasepromoting or disease-limiting functions of the representative MMPs. Moreover, at this point it is not clear whether MMP-3 can be considered a target or anti-target. MMP-3 is known to be able to activate many MMPs by cleavage of the pro-domain and it can be considered as an upstream activation switch. However, based on previously cited data (see Table 8), it is unsure whether blocking MMP-3 would be beneficial for disease development and/or resolution of inflammation in IBD. In contrast, deletion or inhibition of MMP-10 or -19 was determined to be detrimental for disease progression, whereas the opposite was true for MMP-12 and -13 . However, one should be careful with these simplified conclusions, since many factors can influence or stimulate both beneficial and detrimental functions of a single MMP and this can be different both in a certain disease type as well as between different types of diseases.

Although most of the attempts to target MMPs have been performed in a setting of colitis, studying the effects of targeting MMPs or TIMPs on the development of intestinal fibrosis is a relatively novel approach. Intestinal fibrosis and narrowing of the bowel wall are hallmarks of CD. Currently, no adequate therapeutic treatments exist, leaving surgery as the only option for 
patients with fibrostenosing disease. In a recent study, we studied the effect of TIMP-1 deletion on the development of chronic colitis and fibrosis and found that TIMP-1 KO mice developed less fibrosis with lowered amounts of collagen deposition and lowered thickness of mucosal layers (Breynaert \& de Bruyn et al, in press). In contrast, MMP-9 KO mice did not show any difference in development of intestinal fibrosis or collagen deposition/resolution compared to WT mice after induction of chronic DSS-induced colitis (de Bruyn et al, in preparation). Although patients with IBD suffer from chronic intestinal inflammation with aberrant tissue remodeling and blocking MMPs as ECM-remodeling proteases could resolve -in part- these disease processes, we believe that blocking MMPs in a heterogeneous population of IBD patients with complicated individual disease courses and spatiotemporal expressions of MMPs, is at this moment not supported by animal transgenic and knockout studies. However, we do feel that MMP inhibition might be a useful therapeutic strategy in IBD be it in a more shortterm and targeted way of administration. For example, CD patients with fistulizing disease could benefit from additional MMP inhibition in combination with biological treatment to faster control the detrimental breakdown of the mucosa. In addition, patients with fibrostenosing disease for whom currently no adequate therapeutic interventions are available, could benefit from MMP gene delivery or TIMP inhibition. However, processes underlying intestinal fibrosis are not well understood and it is unsure whether inflammation always precedes fibrosis and/or whether fibrosis can be avoided by timely anti-inflammatory interventions. Also, limited amounts of animal models of fibrosis or biomarkers exist to define the exact moment of onset and therefore more preclinical animal studies are warranted.

In conclusion, since a balance between MMPs and TIMPs is crucial, therapeutic interventions targeting MMPS or TIMPs will always present researchers and clinicians with a difficult task of weighting beneficial versus detrimental effects. To further complicate this balancing job, MMPs and TIMPs are part of a bigger protein network and have several ECM-independent 
functions. Careful experimental and preclinical studies are therefore warranted to unravel the complex interaction between MMPs and TIMPs as well as their spatiotemporal expression and function with regard to a particular disease of interest. 


\section{CONCLUSIONS AND FUTURE DIRECTIONS}

Through our literature search we found that only in a few studies the association of SNPs in MMP and TIMP genes with IBD was investigated. Since genetics are an important facet of the pathogenesis of IBD, it is critical to understand whether the occurrence of SNPs in MMPs or TIMPs is associated with an increased risk for disease. Presently the insights provided in many studies on SNPs are limited, because of differences in the ethnicities of the patients who were included. World-wide efforts are underway to genotype more diverse ethnic groups (Liu et al, 2015) and these studies might contribute to a better understanding of the relevance of SNPs in MMPs and TIMPs in IBD. Moreover, as with other inflammatory and autoimmune diseases, it is questioned whether the cumulative small differences in disease susceptibility imposed by many of the 201 IBD-associated SNPs can be therapeutically exploited. The discovery of major genetic factors (such as NOD2, ATG16L1 and IL-23R) may, however, lead to therapeutic implications of specific sub-cohorts of patients. In addition, major associations with specific genes, such as the HLA haplotypes, may help presently in better diagnosis of IBD subtypes. In many studies the expression (mRNA, protein, immunoreactivity) of MMPs and TIMPs has been analyzed in body fluids and tissues of IBD patients and compared with that of healthy control subjects. With regard to gene expression studies, several studies have been previously performed with microarrays (Costello et al, 2005; Dooley et al, 2004; Gillberg et al, 2012; Jimbo et al, 2014; Lakatos et al, 2011; Lawrance et al, 2001; Noble et al, 2008; Noble et al, 2010; Sipos et al, 2011) although only a limited number of MMPs was found to be differentially expressed in IBD patients. In contrast, our group recently performed a broad microarray study on the mucosal gene expression of MMPs and TIMPs following anti-inflammatory treatment (de Bruyn et al, 2014b). In addition, the influence of different types of treatment on the expression of MMPs or TIMPs was investigated in several targeted gene expression studies. However, these studies were only performed on intestinal ex vivo cultures or cell-lines and not 
in blood or mucosal tissue from IBD patients. Interestingly, in two studies the potential of a panel of transcripts to differentially diagnose IBD patients was investigated (Sipos et al, 2011; von Stein et al, 2008). These studies identified that MMP-3, -7 and -9 have the potential to discriminate IBD from non-IBD patients.

With regard to protein expression, the levels of MMPs and TIMPs in blood and mucosal tissue have been reported and, in a number of studies, the cellular localization was demonstrated. In most of these studies, ELISA was used to quantify MMP and TIMP levels. However, the value of this technique needs to be critically analyzed, since ELISA measures immunoreactivity and does neither discriminate between proform, intact, activated and degraded MMPs, between intact or processed TIMPs, nor distinguishes whether the enzyme is free or in complexes with other molecules (e.g. TIMP or NGAL) or whether it is active or neutralized. In this regard, in several other studies Western blot, zymography analysis or activity assays were used. With Western blot analysis, the limitations intrinsic to immunoreactivity remain, however, information with regard to protein size makes it possible to identify intact versus processed and latent versus activated MMP forms. Zymography analysis is mostly used to measure gelatinases (MMP-2 and -9) and with this technique different forms can be distinguished: MMP-9 trimers, latent and activated monomers and complexes with NGAL, latent and activated forms of MMP2 (Vandooren et al, 2013a) (Figure 5). Although both Western blot and zymography can be tuned from semi-quantitative to quantitative methods, these are not valid methods to measure MMP activity. To determine net biological activity in samples, substrate conversion assays are needed. Depending on the used substrate, the activity of a range of MMPs can be determined in a biological sample, whereby the net MMP activity can be measured in the presence of biological inhibitors e.g. TIMPs. However, since several MMPs are able to cleave the same substrate, such assays need to be well controlled (Vandooren et al, 2011). The effect of different types of IBD treatment, including infliximab, on protein expression levels has been investigated 
by a handful of studies (Gao et al, 2007; Geboes et al, 2005; Makitalo et al, 2012b). However, no studies have been performed to investigate the effect of recently developed new types of treatments (e.g. anti-integrin therapy) on the expression of MMPs and TIMPs and on the net activities in the balances between MMPs and TIMPs in IBD.

Since the balance between MMP and TIMP levels determines net MMP activity, it is not sufficient to measure only MMP levels and to associate these with the observed mucosal destruction in IBD. Moreover, in most studies no discrimination is made between latent and activated forms of MMPs. Therefore, more studies are needed on the measurement of both MMPs and TIMPs and to identify the occurrence of different forms of MMPs. Although the imbalance between MMPs and TIMPs is described to play a role in aberrant tissue remodeling in IBD, several recent studies shed new light on an alternative view of this balance (Moore \& Crocker, 2012). An increase in MMPs may be responsible for more tissue destruction; however, TIMPs and MMPs have several independent functions and therefore lowered TIMP levels may also contribute to development of disease. Currently, several groups are further unraveling the role of TIMPs in cell signaling (Edwards et al, 1996; Gomez et al, 1997; Hayakawa et al, 1994; Kobuch et al, 2015; Stetler-Stevenson, 2008).

Regardless of the limitations of several studies, the expression of many MMPs has been shown to be increased in IBD and MMPs may become excellent disease markers, useful for (differential) diagnosis. Moreover, some MMPs have been shown to correlate with disease activity parameters and their use as biomarkers of disease is now widely studied. The detection of the complex between MMP-9 and NGAL, a prototypic neutrophil product, in serum and plasma is useful as IBD disease marker. However, the biological relevance of this aberrant expression in IBD is unclear, mainly the fact whether this is a cause or a consequence of IBD is unknown. In several patient studies, it was observed that elevated MMP expression decreased after anti-inflammatory treatment. We could not corroborate the beneficial effect of MMP 
inhibition in mouse models and, in our hands MMP-9 knockout mice developed similar disease parameters as wild-type mice in both an acute and chronic model of DSS colitis. It remains therefore unclear whether directly targeting MMPs is a valid therapeutic option in IBD. Moreover, the role of MMPs in several normal physiological processes throughout the body is a serious limitation towards development of anti-MMP treatment for IBD. Therefore, additional preclinical and clinical studies are needed to identify whether and eventually which MMPs are cause or consequence of inflammation in IBD. 


\section{ACKNOWLEDGEMENTS}

The authors thank all members of the Laboratory of Immunobiology and the Laboratory of Molecular Immunology, Rega Institute for Medical Research, for practical help. The authors thank Professor Angeles Garcia-Pardo (CSIC, Madrid, Spain), Professor Dylan Edwards (University of East Anglia, Norwich, UK), Professor Edouard Louis (Université de Liège, Belgium) and Professor Jo Van Damme, Professor Xavier Bossuyt and Dr. Annabel Rector (KU Leuven, Belgium) for comments and suggestions. This work is dedicated to Professor Emeritus Karel Geboes and Professor Emeritus Paul Rutgeerts for building the Leuven School for research on IBD.

\section{DECLARATION OF INTEREST}

This study formed part of the doctoral program by Magali de Bruyn, PhD (University of Leuven, March 2016), who was the recipient of a Fellowship from the Agency for Innovation in Science and Technology (IWT, 2013-2016). The present study was supported by grants from the Fund for Scientific Research of Flanders (FWO-Vlaanderen) (grant number: G077513N), the Concerted Research Actions, Belgium (GOA 2013/015) and the Charcot Foundation, Belgium (2016). Estefania Ugarte-Berzal, PhD, and Jennifer Vandooren, $\mathrm{PhD}$ were supported by Postdoctoral Fellowships to the Laboratory of Immunobiology. Ingrid Arijs, $\mathrm{PhD}$ was supported as a postdoctoral fellow and Professor Séverine Vermeire as a Senior Clinical Investigator by the Fund for Scientific Research of Flanders (FWO-Vlaanderen). SV reports following conflicts of interest: grant support, lecture fees and consulting fees from Abbvie, Centocor, MSD, Takeda, Pfizer, Shire, Tillotts Pharma, Hospira, Munipharma, Genentech/Roche. All other authors have no disclosures. 


\section{REFERENCES}

Abraham, C. \& Cho, J. H. (2009) Inflammatory bowel disease. N Engl J Med, 361(21), 2066-78.

Ailenberg, M. \& Sefton, M. V. (2009) Effect of a matrix metalloproteinase sequestering biomaterial on Caco-2 epithelial cell barrier integrity in vitro. Acta Biomater, 5(6), 1898-904.

Allez, M. \& Mayer, L. (2004) Regulatory T cells: peace keepers in the gut. Inflamm Bowel Dis, 10(5), 666-76.

Alutis, M. E., Grundmann, U., Fischer, A., Hagen, U., Kuhl, A. A., Gobel, U. B., Bereswill, S. \& Heimesaat, M. M. (2015a) The Role of Gelatinases in Campylobacter Jejuni Infection of Gnotobiotic Mice. Eur J Microbiol Immunol (Bp), 5(4), 256-67.

Alutis, M. E., Grundmann, U., Hagen, U., Fischer, A., Kuhl, A. A., Gobel, U. B., Bereswill, S. \& Heimesaat, M. M. (2015b) Matrix Metalloproteinase-2 Mediates Intestinal Immunopathogenesis in Campylobacter Jejuni-Infected Infant Mice. Eur J Microbiol Immunol (Bp), 5(3), 188-98.

Anderson, C. A., Boucher, G., Lees, C. W., Franke, A., D'Amato, M., Taylor, K. D., Lee, J. C., Goyette, P., Imielinski, M., Latiano, A., Lagace, C., Scott, R., Amininejad, L., Bumpstead, S., Baidoo, L., Baldassano, R. N., Barclay, M., Bayless, T. M., Brand, S., Buning, C., Colombel, J. F., Denson, L. A., De Vos, M., Dubinsky, M., Edwards, C., Ellinghaus, D., Fehrmann, R. S., Floyd, J. A., Florin, T., Franchimont, D., Franke, L., Georges, M., Glas, J., Glazer, N. L., Guthery, S. L., Haritunians, T., Hayward, N. K., Hugot, J. P., Jobin, G., Laukens, D., Lawrance, I., Lemann, M., Levine, A., Libioulle, C., Louis, E., McGovern, D. P., Milla, M., Montgomery, G. W., Morley, K. I., Mowat, C., Ng, A., Newman, W., Ophoff, R. A., Papi, L., Palmieri, O., Peyrin-Biroulet, L., Panes, J., Phillips, A., Prescott, N. J., Proctor, D. D., Roberts, R., Russell, R., Rutgeerts, P., Sanderson, J., Sans, M., Schumm, P., Seibold, F., Sharma, Y., Simms, L. A., Seielstad, M., Steinhart, A. H., Targan, S. R., van den Berg, L. H., Vatn, M., Verspaget, H., Walters, T., Wijmenga, C., Wilson, D. C., Westra, H. J., Xavier, R. J., Zhao, Z. Z., Ponsioen, C. Y., Andersen, V., Torkvist, L., Gazouli, M., Anagnou, N. P., Karlsen, T. H., Kupcinskas, L., Sventoraityte, J., Mansfield, J. C., Kugathasan, S., Silverberg, M. S., Halfvarson, J., Rotter, J. I., Mathew, C. G., Griffiths, A. M., Gearry, R., Ahmad, T., Brant, S. R., Chamaillard, M., et 
al (2011) Meta-analysis identifies 29 additional ulcerative colitis risk loci, increasing the number of confirmed associations to 47. Nat Genet, 43(3), 246-52.

Andoh, A., Kuzuoka, H., Tsujikawa, T., Nakamura, S., Hirai, F., Suzuki, Y., Matsui, T., Fujiyama, Y. \& Matsumoto, T. (2012) Multicenter analysis of fecal microbiota profiles in Japanese patients with Crohn's disease. J Gastroenterol, 47(12), 1298-307.

Annahazi, A., Molnar, T., Farkas, K., Rosztoczy, A., Izbeki, F., Gecse, K., Inczefi, O., Nagy, F., Foldesi, I., Szucs, M., Dabek, M., Ferrier, L., Theodorou, V., Bueno, L., Wittmann, T. \& Roka, R. (2013) Fecal MMP-9: A New Noninvasive Differential Diagnostic and Activity Marker in Ulcerative Colitis. Inflamm Bowel Dis, 19(2), 316-20.

Arihiro, S., Ohtani, H., Hiwatashi, N., Torii, A., Sorsa, T. \& Nagura, H. (2001) Vascular smooth muscle cells and pericytes express MMP-1, MMP-9, TIMP-1 and type I procollagen in inflammatory bowel disease. Histopathology, 39(1), 50-9.

Arijs, I., De Hertogh, G., Lemaire, K., Quintens, R., Van Lommel, L., Van Steen, K., Leemans, P., Cleynen, I., Van Assche, G., Vermeire, S., Geboes, K., Schuit, F. \& Rutgeerts, P. (2009) Mucosal gene expression of antimicrobial peptides in inflammatory bowel disease. PLoS One, 4(11), e7984.

Axelsson, L. G., Landstrom, E., Goldschmidt, T. J., Gronberg, A. \& Bylund-Fellenius, A. C. (1996) Dextran sulfate sodium (DSS) induced experimental colitis in immunodeficient mice: effects in CD4(+) -cell depleted, athymic and NK-cell depleted SCID mice. Inflamm Res, 45(4), 181-91.

Baert, F., Noman, M., Vermeire, S., Van Assche, G., G, D. H., Carbonez, A. \& Rutgeerts, P. (2003) Influence of immunogenicity on the long-term efficacy of infliximab in Crohn's disease. $N$ Engl J Med, 348(7), 601-8.

Bailey, C. J., Hembry, R. M., Alexander, A., Irving, M. H., Grant, M. E. \& Shuttleworth, C. A. (1994) Distribution of the matrix metalloproteinases stromelysin, gelatinases A and B, and collagenase in Crohn's disease and normal intestine. J Clin Pathol, 47(2), 113-6.

Bailon, E., Ugarte-Berzal, E., Amigo-Jimenez, I., Van den Steen, P., Opdenakker, G., Garcia-Marco, J. A. \& Garcia-Pardo, A. (2014) Overexpression of progelatinase B/proMMP-9 affects migration 
regulatory pathways and impairs chronic lymphocytic leukemia cell homing to bone marrow and spleen. J Leukoc Biol, 96(2), 185-99.

Baker, A. H., Edwards, D. R. \& Murphy, G. (2002) Metalloproteinase inhibitors: biological actions and therapeutic opportunities. J Cell Sci, 115(Pt 19), 3719-27.

Bamias, G., Dahman, M. I., Arseneau, K. O., Guanzon, M., Gruska, D., Pizarro, T. T. \& Cominelli, F. (2013) Intestinal-specific TNFalpha overexpression induces Crohn's-like ileitis in mice. PLoS One, 8(8), e72594.

Banerjee, A., Bizzaro, D., Burra, P., Di Liddo, R., Pathak, S., Arcidiacono, D., Cappon, A., Bo, P., Conconi, M. T., Crescenzi, M., Pinna, C. M., Parnigotto, P. P., Alison, M. R., Sturniolo, G. C., D'Inca, R. \& Russo, F. P. (2015) Umbilical cord mesenchymal stem cells modulate dextran sulfate sodium induced acute colitis in immunodeficient mice. Stem Cell Res Ther, 6, 79.

Baugh, M. D., Perry, M. J., Hollander, A. P., Davies, D. R., Cross, S. S., Lobo, A. J., Taylor, C. J. \& Evans, G. S. (1999) Matrix metalloproteinase levels are elevated in inflammatory bowel disease. Gastroenterology, 117(4), 814-22.

Becker, C., Neurath, M. F. \& Wirtz, S. (2015) The Intestinal Microbiota in Inflammatory Bowel Disease. Ilar j, 56(2), 192-204.

Bennike, T., Birkelund, S., Stensballe, A. \& Andersen, V. (2014) Biomarkers in inflammatory bowel diseases: current status and proteomics identification strategies. World J Gastroenterol, 20(12), 323144.

Berrebi, D., Besnard, M., Fromont-Hankard, G., Paris, R., Mougenot, J. F., De Lagausie, P., Emilie, D., Cezard, J. P., Navarro, J. \& Peuchmaur, M. (1998) Interleukin-12 expression is focally enhanced in the gastric mucosa of pediatric patients with Crohn's disease. Am J Pathol, 152(3), 667-72.

Bhandari, B. R., Fogel, R., Onken, J., Yen, E. H., Kanwar, B., Subramanian, G. M., McHutchison, J. G., Lee, S. D., Feagan, B. G. \& Sandborn, W. (2015) Tu2056 Safety and Efficacy of GS-5745 an AntiMatrix Metalloproteinase 9 (MMP) Monoclonal Antibody in Patients With Moderately to Severely Active Ulcerative Colitis. Gastroenterology, 148(4). 
Bhol, K. C. \& Schechter, P. J. (2007) Effects of nanocrystalline silver (NPI 32101) in a rat model of ulcerative colitis. Dig Dis Sci, 52(10), 2732-42.

Bister, V. O., Salmela, M. T., Karjalainen-Lindsberg, M. L., Uria, J., Lohi, J., Puolakkainen, P., LopezOtin, C. \& Saarialho-Kere, U. (2004) Differential expression of three matrix metalloproteinases, MMP19, MMP-26, and MMP-28, in normal and inflamed intestine and colon cancer. Dig Dis Sci, 49(4), 65361.

Bjerrum, J. T., Nyberg, C., Olsen, J. \& Nielsen, O. H. (2014) Assessment of the validity of a multigene analysis in the diagnostics of inflammatory bowel disease. J Intern Med, 275(5), 484-93.

Boschetti, G., Garnero, P., Moussata, D., Cuerq, C., Preaudat, C., Duclaux-Loras, R., Mialon, A., Drai, J., Flourie, B. \& Nancey, S. (2015) Accuracies of serum and fecal S100 proteins (calprotectin and calgranulin C) to predict the response to TNF antagonists in patients with Crohn's disease. Inflamm Bowel Dis, 21(2), 331-6.

Brand, S. (2009) Crohn's disease: Th1, Th17 or both? The change of a paradigm: new immunological and genetic insights implicate Th17 cells in the pathogenesis of Crohn's disease. Gut, 58(8), 1152-67.

Brauer, R., Tureckova, J., Kanchev, I., Khoylou, M., Skarda, J., Prochazka, J., Spoutil, F., Beck, I. M., Zbodakova, O., Kasparek, P., Korinek, V., Chalupsky, K., Karhu, T., Herzig, K. H., Hajduch, M., Gregor, M. \& Sedlacek, R. (2015) MMP-19 deficiency causes aggravation of colitis due to defects in innate immune cell function. Mucosal Immunol.

Brazil, J. C., Louis, N. A. \& Parkos, C. A. (2013) The role of polymorphonuclear leukocyte trafficking in the perpetuation of inflammation during inflammatory bowel disease. Inflamm Bowel Dis, 19(7), $1556-65$.

Brew, K., Dinakarpandian, D. \& Nagase, H. (2000) Tissue inhibitors of metalloproteinases: evolution, structure and function. Biochim Biophys Acta, 1477(1-2), 267-83.

Breynaert, C., Dresselaers, T., Perrier, C., Arijs, I., Cremer, J., Van Lommel, L., Van Steen, K., Ferrante, M., Schuit, F., Vermeire, S., Rutgeerts, P., Himmelreich, U., Ceuppens, J. L., Geboes, K. \& Van Assche, G. (2013) Unique gene expression and MR T2 relaxometry patterns define chronic murine dextran 
sodium sulphate colitis as a model for connective tissue changes in human Crohn's disease. PLoS One, 8(7), e68876.

Butler, G. S., Apte, S. S., Willenbrock, F. \& Murphy, G. (1999) Human tissue inhibitor of metalloproteinases 3 interacts with both the $\mathrm{N}$ - and C-terminal domains of gelatinases $\mathrm{A}$ and $\mathrm{B}$. Regulation by polyanions. J Biol Chem, 274(16), 10846-51.

Cadwell, K., Liu, J. Y., Brown, S. L., Miyoshi, H., Loh, J., Lennerz, J. K., Kishi, C., Kc, W., Carrero, J. A., Hunt, S., Stone, C. D., Brunt, E. M., Xavier, R. J., Sleckman, B. P., Li, E., Mizushima, N., Stappenbeck, T. S. \& Virgin, H. W. t. (2008) A key role for autophagy and the autophagy gene Atg1611 in mouse and human intestinal Paneth cells. Nature, 456(7219), 259-63.

Castaneda, F. E., Walia, B., Vijay-Kumar, M., Patel, N. R., Roser, S., Kolachala, V. L., Rojas, M., Wang, L., Oprea, G., Garg, P., Gewirtz, A. T., Roman, J., Merlin, D. \& Sitaraman, S. V. (2005) Targeted deletion of metalloproteinase 9 attenuates experimental colitis in mice: central role of epithelial-derived MMP. Gastroenterology, 129(6), 1991-2008.

Cauwe, B., Martens, E., Proost, P. \& Opdenakker, G. (2009) Multidimensional degradomics identifies systemic autoantigens and intracellular matrix proteins as novel gelatinase B/MMP-9 substrates. Integr Biol (Camb), 1(5-6), 404-26.

Cauwe, B. \& Opdenakker, G. (2010) Intracellular substrate cleavage: a novel dimension in the biochemistry, biology and pathology of matrix metalloproteinases. Crit Rev Biochem Mol Biol, 45(5), $351-423$.

Cauwe, B., Van den Steen, P. E. \& Opdenakker, G. (2007) The biochemical, biological, and pathological kaleidoscope of cell surface substrates processed by matrix metalloproteinases. Crit Rev Biochem Mol Biol, 42(3), 113-85.

Chakraborti, S., Mandal, M., Das, S., Mandal, A. \& Chakraborti, T. (2003) Regulation of matrix metalloproteinases: an overview. Mol Cell Biochem, 253(1-2), 269-85. 
Chan, Y. R., Liu, J. S., Pociask, D. A., Zheng, M., Mietzner, T. A., Berger, T., Mak, T. W., Clifton, M. C., Strong, R. K., Ray, P. \& Kolls, J. K. (2009) Lipocalin 2 is required for pulmonary host defense against Klebsiella infection. J Immunol, 182(8), 4947-56.

Chassaing, B., Srinivasan, G., Delgado, M. A., Young, A. N., Gewirtz, A. T. \& Vijay-Kumar, M. (2012) Fecal lipocalin 2, a sensitive and broadly dynamic non-invasive biomarker for intestinal inflammation. PLoS One, 7(9), e44328.

Chen, K. C., Liao, Y. C., Hsieh, I. C., Wang, Y. S., Hu, C. Y. \& Juo, S. H. (2012) OxLDL causes both epigenetic modification and signaling regulation on the microRNA-29b gene: novel mechanisms for cardiovascular diseases. J Mol Cell Cardiol, 52(3), 587-95.

Chen, M., Peyrin-Biroulet, L., George, A., Coste, F., Bressenot, A., Bossenmeyer-Pourie, C., Alberto, J. M., Xia, B., Namour, B. \& Gueant, J. L. (2011) Methyl deficient diet aggravates experimental colitis in rats. J Cell Mol Med, 15(11), 2486-97.

Chirco, R., Liu, X. W., Jung, K. K. \& Kim, H. R. (2006) Novel functions of TIMPs in cell signaling. Cancer Metastasis Rev, 25(1), 99-113.

Cho, H. J., Cho, J. Y., Rhee, M. H. \& Park, H. J. (2007) Cordycepin (3'-deoxyadenosine) inhibits human platelet aggregation in a cyclic AMP- and cyclic GMP-dependent manner. Eur J Pharmacol, 558(1-3), 43-51.

Cho, J. H. (2008) The genetics and immunopathogenesis of inflammatory bowel disease. Nat Rev Immunol, 8(6), 458-66.

Claramunt, R. M., Bouissane, L., Cabildo, M. P., Cornago, M. P., Elguero, J., Radziwon, A. \& Medina, C. (2009) Synthesis and biological evaluation of curcuminoid pyrazoles as new therapeutic agents in inflammatory bowel disease: effect on matrix metalloproteinases. Bioorg Med Chem, 17(3), 1290-6.

Cleynen, I., Boucher, G., Jostins, L., Schumm, L. P., Zeissig, S., Ahmad, T., Andersen, V., Andrews, J. M., Annese, V., Brand, S., Brant, S. R., Cho, J. H., Daly, M. J., Dubinsky, M., Duerr, R. H., Ferguson, L. R., Franke, A., Gearry, R. B., Goyette, P., Hakonarson, H., Halfvarson, J., Hov, J. R., Huang, H., Kennedy, N. A., Kupcinskas, L., Lawrance, I. C., Lee, J. C., Satsangi, J., Schreiber, S., Theatre, E., van 
der Meulen-de Jong, A. E., Weersma, R. K., Wilson, D. C., Parkes, M., Vermeire, S., Rioux, J. D., Mansfield, J., Silverberg, M. S., Radford-Smith, G., McGovern, D. P., Barrett, J. C. \& Lees, C. W. (2016) Inherited determinants of Crohn's disease and ulcerative colitis phenotypes: a genetic association study. Lancet, 387(10014), 156-67.

Cleynen, I., Gonzalez, J. R., Figueroa, C., Franke, A., McGovern, D., Bortlik, M., Crusius, B. J., Vecchi, M., Artieda, M., Szczypiorska, M., Bethge, J., Arteta, D., Ayala, E., Danese, S., van Hogezand, R. A., Panes, J., Pena, S. A., Lukas, M., Jewell, D. P., Schreiber, S., Vermeire, S. \& Sans, M. (2013) Genetic factors conferring an increased susceptibility to develop Crohn's disease also influence disease phenotype: results from the IBDchip European Project. Gut, 62(11), 1556-65.

Collazo, C. M., Yap, G. S., Sempowski, G. D., Lusby, K. C., Tessarollo, L., Vande Woude, G. F., Sher, A. \& Taylor, G. A. (2001) Inactivation of LRG-47 and IRG-47 reveals a family of interferon gammainducible genes with essential, pathogen-specific roles in resistance to infection. $J$ Exp Med, 194(2), $181-8$

Colombel, J. F., Sandborn, W. J., Reinisch, W., Mantzaris, G. J., Kornbluth, A., Rachmilewitz, D., Lichtiger, S., D'Haens, G., Diamond, R. H., Broussard, D. L., Tang, K. L., van der Woude, C. J. \& Rutgeerts, P. (2010) Infliximab, azathioprine, or combination therapy for Crohn's disease. N Engl J Med, 362(15), 1383-95.

Colombel, J. F., Sandborn, W. J., Rutgeerts, P., Enns, R., Hanauer, S. B., Panaccione, R., Schreiber, S., Byczkowski, D., Li, J., Kent, J. D. \& Pollack, P. F. (2007) Adalimumab for maintenance of clinical response and remission in patients with Crohn's disease: the CHARM trial. Gastroenterology, 132(1), $52-65$.

Coskun, M., Steenholdt, C., de Boer, N. K. \& Nielsen, O. H. (2016) Pharmacology and Optimization of Thiopurines and Methotrexate in Inflammatory Bowel Disease. Clin Pharmacokinet, 55(3), 257-74.

Costello, C. M., Mah, N., Hasler, R., Rosenstiel, P., Waetzig, G. H., Hahn, A., Lu, T., Gurbuz, Y., Nikolaus, S., Albrecht, M., Hampe, J., Lucius, R., Kloppel, G., Eickhoff, H., Lehrach, H., Lengauer, T. 
\& Schreiber, S. (2005) Dissection of the inflammatory bowel disease transcriptome using genome-wide cDNA microarrays. PLoS Med, 2(8), e199.

Coussens, L. M., Fingleton, B. \& Matrisian, L. M. (2002) Matrix metalloproteinase inhibitors and cancer: trials and tribulations. Science, 295(5564), 2387-92.

Cuzzocrea, S., Mazzon, E., Serraino, I., Lepore, V., Terranova, M. L., Ciccolo, A. \& Caputi, A. P. (2001) Melatonin reduces dinitrobenzene sulfonic acid-induced colitis. J Pineal Res, 30(1), 1-12.

D'Haens, G., Van Deventer, S., Van Hogezand, R., Chalmers, D., Kothe, C., Baert, F., Braakman, T., Schaible, T., Geboes, K. \& Rutgeerts, P. (1999) Endoscopic and histological healing with infliximab anti-tumor necrosis factor antibodies in Crohn's disease: A European multicenter trial. Gastroenterology, 116(5), 1029-34.

Danese, S. (2008) Mechanisms of action of infliximab in inflammatory bowel disease: an antiinflammatory multitasker. Dig Liver Dis, 40 Suppl 2, S225-8.

Danese, S., Sans, M. \& Fiocchi, C. (2004) Inflammatory bowel disease: the role of environmental factors. Autoimmun Rev, 3(5), 394-400.

Danese, S., Sgambato, A., Papa, A., Scaldaferri, F., Pola, R., Sans, M., Lovecchio, M., Gasbarrini, G., Cittadini, A. \& Gasbarrini, A. (2005) Homocysteine triggers mucosal microvascular activation in inflammatory bowel disease. Am J Gastroenterol, 100(4), 886-95.

Danese, S., Vuitton, L. \& Peyrin-Biroulet, L. (2015) Biologic agents for IBD: practical insights. Nat Rev Gastroenterol Hepatol, 12(9), 537-45.

de Bruyn, M., Arijs, I., De Hertogh, G., Ferrante, M., Van Assche, G., Rutgeerts, P., Vermeire, S. \& Opdenakker, G. (2015) Serum Neutrophil Gelatinase B-associated Lipocalin and Matrix Metalloproteinase-9 Complex as a Surrogate Marker for Mucosal Healing in Patients with Crohn's Disease. J Crohns Colitis, 9(12), 1079-87.

de Bruyn, M., Arijs, I., Wollants, W. J., Machiels, K., Van Steen, K., Van Assche, G., Ferrante, M., Rutgeerts, P., Vermeire, S. \& Opdenakker, G. (2014a) Neutrophil gelatinase B-associated lipocalin and 
matrix metalloproteinase-9 complex as a surrogate serum marker of mucosal healing in ulcerative colitis. Inflamm Bowel Dis, 20(7), 1198-207.

de Bruyn, M., Machiels, K., Vandooren, J., Lemmens, B., Van Lommel, L., Breynaert, C., Van der Goten, J., Staelens, D., Billiet, T., De Hertogh, G., Ferrante, M., Van Assche, G., Vermeire, S., Opdenakker, G., Schuit, F., Rutgeerts, P. \& Arijs, I. (2014b) Infliximab restores the dysfunctional matrix remodeling protein and growth factor gene expression in patients with inflammatory bowel disease. Inflamm Bowel Dis, 20(2), 339-52.

De Cruz, P., Kamm, M. A., Prideaux, L., Allen, P. B. \& Desmond, P. V. (2012) Postoperative recurrent luminal Crohn's disease: a systematic review. Inflamm Bowel Dis, 18(4), 758-77.

Deryugina, E. I., Bourdon, M. A., Jungwirth, K., Smith, J. W. \& Strongin, A. Y. (2000) Functional activation of integrin alpha $\mathrm{V}$ beta 3 in tumor cells expressing membrane-type 1 matrix metalloproteinase. Int J Cancer, 86(1), 15-23.

Devy, L. \& Dransfield, D. T. (2011) New Strategies for the Next Generation of MatrixMetalloproteinase Inhibitors: Selectively Targeting Membrane-Anchored MMPs with Therapeutic Antibodies. Biochem Res Int, 2011, 191670.

Di Paola, R., Esposito, E., Mazzon, E., Riccardi, L., Caminiti, R., Dal Toso, R., Pressi, G. \& Cuzzocrea, S. (2009) Teupolioside, a phenylpropanoid glycosides of Ajuga reptans, biotechnologically produced by IRBN22 plant cell line, exerts beneficial effects on a rodent model of colitis. Biochem Pharmacol, 77(5), 845-57.

Di Sabatino, A., Pender, S. L., Jackson, C. L., Prothero, J. D., Gordon, J. N., Picariello, L., Rovedatti, L., Docena, G., Monteleone, G., Rampton, D. S., Tonelli, F., Corazza, G. R. \& MacDonald, T. T. (2007) Functional modulation of Crohn's disease myofibroblasts by anti-tumor necrosis factor antibodies. Gastroenterology, 133(1), 137-49.

Di Sebastiano, P., di Mola, F. F., Artese, L., Rossi, C., Mascetta, G., Pernthaler, H. \& Innocenti, P. (2001) Beneficial effects of Batimastat (BB-94), a matrix metalloproteinase inhibitor, in rat experimental colitis. Digestion, 63(4), 234-9. 
Ding, H., Gan, H. Z., Fan, W. J., Cao, L. Y., Xu, J. M. \& Mei, Q. (2015) Homocysteine promotes intestinal fibrosis in rats with trinitrobenzene sulfonic acid-induced colitis. Dig Dis Sci, 60(2), 375-81.

Ding, H., Mei, Q., Gan, H. Z., Cao, L. Y., Liu, X. C. \& Xu, J. M. (2014) Effect of homocysteine on intestinal permeability in rats with experimental colitis, and its mechanism. Gastroenterol Rep (Oxf), 2(3), 215-20.

Docherty, A. J., Lyons, A., Smith, B. J., Wright, E. M., Stephens, P. E., Harris, T. J., Murphy, G. \& Reynolds, J. J. (1985) Sequence of human tissue inhibitor of metalloproteinases and its identity to erythroid-potentiating activity. Nature, 318(6041), 66-9.

Dooley, T. P., Curto, E. V., Reddy, S. P., Davis, R. L., Lambert, G. W., Wilborn, T. W. \& Elson, C. O. (2004) Regulation of gene expression in inflammatory bowel disease and correlation with IBD drugs: screening by DNA microarrays. Inflamm Bowel Dis, 10(1), 1-14.

Dufour, A. \& Overall, C. M. (2013) Missing the target: matrix metalloproteinase antitargets in inflammation and cancer. Trends Pharmacol Sci, 34(4), 233-42.

Edwards, D. R., Beaudry, P. P., Laing, T. D., Kowal, V., Leco, K. J., Leco, P. A. \& Lim, M. S. (1996) The roles of tissue inhibitors of metalloproteinases in tissue remodelling and cell growth. Int J Obes Relat Metab Disord, 20 Suppl 3, S9-15.

Edwards, D. R., Murphy, G., Reynolds, J. J., Whitham, S. E., Docherty, A. J., Angel, P. \& Heath, J. K. (1987) Transforming growth factor beta modulates the expression of collagenase and metalloproteinase inhibitor. Embo j, 6(7), 1899-904.

Eguchi, T., Kubota, S., Kawata, K., Mukudai, Y., Uehara, J., Ohgawara, T., Ibaragi, S., Sasaki, A., Kuboki, T. \& Takigawa, M. (2008) Novel transcription-factor-like function of human matrix metalloproteinase 3 regulating the CTGF/CCN2 gene. Mol Cell Biol, 28(7), 2391-413.

Ellinghaus, D., Jostins, L., Spain, S. L., Cortes, A., Bethune, J., Han, B., Park, Y. R., Raychaudhuri, S., Pouget, J. G., Hubenthal, M., Folseraas, T., Wang, Y., Esko, T., Metspalu, A., Westra, H. J., Franke, L., Pers, T. H., Weersma, R. K., Collij, V., D'Amato, M., Halfvarson, J., Jensen, A. B., Lieb, W., Degenhardt, F., Forstner, A. J., Hofmann, A., Schreiber, S., Mrowietz, U., Juran, B. D., Lazaridis, K. 
N., Brunak, S., Dale, A. M., Trembath, R. C., Weidinger, S., Weichenthal, M., Ellinghaus, E., Elder, J. T., Barker, J. N., Andreassen, O. A., McGovern, D. P., Karlsen, T. H., Barrett, J. C., Parkes, M., Brown, M. A. \& Franke, A. (2016) Analysis of five chronic inflammatory diseases identifies 27 new associations and highlights disease-specific patterns at shared loci. Nat Genet.

Elson, C. O., Cong, Y., McCracken, V. J., Dimmitt, R. A., Lorenz, R. G. \& Weaver, C. T. (2005) Experimental models of inflammatory bowel disease reveal innate, adaptive, and regulatory mechanisms of host dialogue with the microbiota. Immunol Rev, 206, 260-76.

Epstein, J., Docena, G., MacDonald, T. T. \& Sanderson, I. R. (2010) Curcumin suppresses p38 mitogenactivated protein kinase activation, reduces IL-1beta and matrix metalloproteinase- 3 and enhances IL10 in the mucosa of children and adults with inflammatory bowel disease. Br J Nutr, 103(6), 824-32.

Esposito, E., Mazzon, E., Riccardi, L., Caminiti, R., Meli, R. \& Cuzzocrea, S. (2008) Matrix metalloproteinase- 9 and metalloproteinase- 2 activity and expression is reduced by melatonin during experimental colitis. J Pineal Res, 45(2), 166-73.

Farkas, K., Sarodi, Z., Balint, A., Foldesi, I., Tiszlavicz, L., Szucs, M., Nyari, T., Tajti, J., Nagy, F., Szepes, Z., Bor, R., Annahazi, A., Roka, R. \& Molnar, T. (2015) The diagnostic value of a new fecal marker, matrix metalloprotease-9, in different types of inflammatory bowel diseases. J Crohns Colitis, 9(3), 231-7.

Faubion, W. A., Jr., Fletcher, J. G., O'Byrne, S., Feagan, B. G., de Villiers, W. J., Salzberg, B., Plevy, S., Proctor, D. D., Valentine, J. F., Higgins, P. D., Harris, J. M., Diehl, L., Wright, L., Tew, G. W., Luca, D., Basu, K. \& Keir, M. E. (2013) EMerging BiomARKers in Inflammatory Bowel Disease (EMBARK) study identifies fecal calprotectin, serum MMP9, and serum IL-22 as a novel combination of biomarkers for Crohn's disease activity: role of cross-sectional imaging. Am J Gastroenterol, 108(12), 1891-900.

Feagan, B. G. \& Macdonald, J. K. (2012a) Oral 5-aminosalicylic acid for induction of remission in ulcerative colitis. Cochrane Database Syst Rev, 10, Cd000543.

Feagan, B. G. \& Macdonald, J. K. (2012b) Oral 5-aminosalicylic acid for maintenance of remission in ulcerative colitis. Cochrane Database Syst Rev, 10, Cd000544. 
Feagan, B. G., Rutgeerts, P., Sands, B. E., Hanauer, S., Colombel, J. F., Sandborn, W. J., Van Assche, G., Axler, J., Kim, H. J., Danese, S., Fox, I., Milch, C., Sankoh, S., Wyant, T., Xu, J. \& Parikh, A. (2013) Vedolizumab as induction and maintenance therapy for ulcerative colitis. N Engl J Med, 369(8), 699710.

Fields, G. B. (2015) New strategies for targeting matrix metalloproteinases. Matrix Biol, 44-46, 239-46.

Fingleton, B. (2003) Matrix metalloproteinase inhibitors for cancer therapy:the current situation and future prospects. Expert Opin Ther Targets, 7(3), 385-97.

Flo, T. H., Smith, K. D., Sato, S., Rodriguez, D. J., Holmes, M. A., Strong, R. K., Akira, S. \& Aderem, A. (2004) Lipocalin 2 mediates an innate immune response to bacterial infection by sequestrating iron. Nature, 432(7019), 917-21.

Ford, A. C., Bernstein, C. N., Khan, K. J., Abreu, M. T., Marshall, J. K., Talley, N. J. \& Moayyedi, P. (2011) Glucocorticosteroid therapy in inflammatory bowel disease: systematic review and metaanalysis. Am J Gastroenterol, 106(4), 590-9; quiz 600.

Frank, D. N., St Amand, A. L., Feldman, R. A., Boedeker, E. C., Harpaz, N. \& Pace, N. R. (2007) Molecular-phylogenetic characterization of microbial community imbalances in human inflammatory bowel diseases. Proc Natl Acad Sci U S A, 104(34), 13780-5.

Franke, A., McGovern, D. P., Barrett, J. C., Wang, K., Radford-Smith, G. L., Ahmad, T., Lees, C. W., Balschun, T., Lee, J., Roberts, R., Anderson, C. A., Bis, J. C., Bumpstead, S., Ellinghaus, D., Festen, E. M., Georges, M., Green, T., Haritunians, T., Jostins, L., Latiano, A., Mathew, C. G., Montgomery, G. W., Prescott, N. J., Raychaudhuri, S., Rotter, J. I., Schumm, P., Sharma, Y., Simms, L. A., Taylor, K. D., Whiteman, D., Wijmenga, C., Baldassano, R. N., Barclay, M., Bayless, T. M., Brand, S., Buning, C., Cohen, A., Colombel, J. F., Cottone, M., Stronati, L., Denson, T., De Vos, M., D'Inca, R., Dubinsky, M., Edwards, C., Florin, T., Franchimont, D., Gearry, R., Glas, J., Van Gossum, A., Guthery, S. L., Halfvarson, J., Verspaget, H. W., Hugot, J. P., Karban, A., Laukens, D., Lawrance, I., Lemann, M., Levine, A., Libioulle, C., Louis, E., Mowat, C., Newman, W., Panes, J., Phillips, A., Proctor, D. D., Regueiro, M., Russell, R., Rutgeerts, P., Sanderson, J., Sans, M., Seibold, F., Steinhart, A. H., Stokkers, 
P. C., Torkvist, L., Kullak-Ublick, G., Wilson, D., Walters, T., Targan, S. R., Brant, S. R., Rioux, J. D., D'Amato, M., Weersma, R. K., Kugathasan, S., Griffiths, A. M., Mansfield, J. C., Vermeire, S., Duerr, R. H., Silverberg, M. S., Satsangi, J., Schreiber, S., Cho, J. H., Annese, V., Hakonarson, H., Daly, M. J. \& Parkes, M. (2010) Genome-wide meta-analysis increases to 71 the number of confirmed Crohn's disease susceptibility loci. Nat Genet, 42(12), 1118-25.

Freise, C., Ruehl, M., Erben, U., Farndale, R. W., Somasundaram, R. \& Heimesaat, M. M. (2012) The synthetic hydroxyproline-containing collagen analogue (Gly-Pro-Hyp)10 promotes enzymatic activity of matrixmetalloproteinase-2 in vitro. Eur J Microbiol Immunol (Bp), 2(3), 186-91.

Freour, T., Jarry, A., Bach-Ngohou, K., Dejoie, T., Bou-Hanna, C., Denis, M. G., Mosnier, J. F., Laboisse, C. L. \& Masson, D. (2009) TACE inhibition amplifies TNF-alpha-mediated colonic epithelial barrier disruption. Int J Mol Med, 23(1), 41-8.

Frolkis, A. D., Dykeman, J., Negron, M. E., Debruyn, J., Jette, N., Fiest, K. M., Frolkis, T., Barkema, H. W., Rioux, K. P., Panaccione, R., Ghosh, S., Wiebe, S. \& Kaplan, G. G. (2013) Risk of surgery for inflammatory bowel diseases has decreased over time: a systematic review and meta-analysis of population-based studies. Gastroenterology, 145(5), 996-1006.

Frolkis, A. D., Lipton, D. S., Fiest, K. M., Negron, M. E., Dykeman, J., deBruyn, J., Jette, N., Frolkis, T., Rezaie, A., Seow, C. H., Panaccione, R., Ghosh, S. \& Kaplan, G. G. (2014) Cumulative incidence of second intestinal resection in Crohn's disease: a systematic review and meta-analysis of populationbased studies. Am J Gastroenterol, 109(11), 1739-48.

Gan, X., Wong, B., Wright, S. D. \& Cai, T. Q. (2001) Production of matrix metalloproteinase-9 in CaCO-2 cells in response to inflammatory stimuli. J Interferon Cytokine Res, 21(2), 93-8.

Gao, Q., Meijer, M. J., Kubben, F. J., Sier, C. F., Kruidenier, L., van Duijn, W., van den Berg, M., van Hogezand, R. A., Lamers, C. B. \& Verspaget, H. W. (2005) Expression of matrix metalloproteinases-2 and -9 in intestinal tissue of patients with inflammatory bowel diseases. Dig Liver Dis, 37(8), 584-92.

Gao, Q., Meijer, M. J., Schluter, U. G., van Hogezand, R. A., van der Zon, J. M., van den Berg, M., van Duijn, W., Lamers, C. B. \& Verspaget, H. W. (2007) Infliximab treatment influences the serological 
expression of matrix metalloproteinase (MMP)-2 and -9 in Crohn's disease. Inflamm Bowel Dis, 13(6), 693-702.

Garg, P., Ravi, A., Patel, N. R., Roman, J., Gewirtz, A. T., Merlin, D. \& Sitaraman, S. V. (2007) Matrix metalloproteinase-9 regulates MUC-2 expression through its effect on goblet cell differentiation. Gastroenterology, 132(5), 1877-89.

Garg, P., Rojas, M., Ravi, A., Bockbrader, K., Epstein, S., Vijay-Kumar, M., Gewirtz, A. T., Merlin, D. \& Sitaraman, S. V. (2006) Selective ablation of matrix metalloproteinase-2 exacerbates experimental colitis: contrasting role of gelatinases in the pathogenesis of colitis. J Immunol, 177(6), 4103-12.

Garg, P., Sarma, D., Jeppsson, S., Patel, N. R., Gewirtz, A. T., Merlin, D. \& Sitaraman, S. V. (2010) Matrix metalloproteinase-9 functions as a tumor suppressor in colitis-associated cancer. Cancer Res, 70(2), 792-801.

Garg, P., Vijay-Kumar, M., Wang, L., Gewirtz, A. T., Merlin, D. \& Sitaraman, S. V. (2009) Matrix metalloproteinase-9-mediated tissue injury overrides the protective effect of matrix metalloproteinase-2 during colitis. Am J Physiol Gastrointest Liver Physiol, 296(2), G175-84.

Garrett, W. S., Lord, G. M., Punit, S., Lugo-Villarino, G., Mazmanian, S. K., Ito, S., Glickman, J. N. \& Glimcher, L. H. (2007) Communicable ulcerative colitis induced by T-bet deficiency in the innate immune system. Cell, 131(1), 33-45.

Garrido-Mesa, N., Utrilla, P., Comalada, M., Zorrilla, P., Garrido-Mesa, J., Zarzuelo, A., RodriguezCabezas, M. E. \& Galvez, J. (2011) The association of minocycline and the probiotic Escherichia coli Nissle 1917 results in an additive beneficial effect in a DSS model of reactivated colitis in mice. Biochem Pharmacol, 82(12), 1891-900.

Geboes, K., Rutgeerts, P., Opdenakker, G., Olson, A., Patel, K., Wagner, C. L. \& Marano, C. W. (2005) Endoscopic and histologic evidence of persistent mucosal healing and correlation with clinical improvement following sustained infliximab treatment for Crohn's disease. Curr Med Res Opin, 21(11), $1741-54$ 
Gill, S. E., Kassim, S. Y., Birkland, T. P. \& Parks, W. C. (2010) Mouse models of MMP and TIMP function. Methods Mol Biol, 622, 31-52.

Gillberg, L., Varsanyi, M., Sjostrom, M., Lordal, M., Lindholm, J. \& Hellstrom, P. M. (2012) Nitric oxide pathway-related gene alterations in inflammatory bowel disease. Scand J Gastroenterol, 47(11), 1283-97.

Giner, E., Andujar, I., Recio, M. C., Rios, J. L., Cerda-Nicolas, J. M. \& Giner, R. M. (2011) Oleuropein ameliorates acute colitis in mice. J Agric Food Chem, 59(24), 12882-92.

Gomez, D. E., Alonso, D. F., Yoshiji, H. \& Thorgeirsson, U. P. (1997) Tissue inhibitors of metalloproteinases: structure, regulation and biological functions. Eur J Cell Biol, 74(2), 111-22.

Gomis-Ruth, F. X., Maskos, K., Betz, M., Bergner, A., Huber, R., Suzuki, K., Yoshida, N., Nagase, H., Brew, K., Bourenkov, G. P., Bartunik, H. \& Bode, W. (1997) Mechanism of inhibition of the human matrix metalloproteinase stromelysin-1 by TIMP-1. Nature, 389(6646), 77-81.

Gordon, J. N., Pickard, K. M., Di Sabatino, A., Prothero, J. D., Pender, S. L., Goggin, P. M. \& MacDonald, T. T. (2008) Matrix metalloproteinase-3 production by gut IgG plasma cells in chronic inflammatory bowel disease. Inflamm Bowel Dis, 14(2), 195-203.

Gordon, J. N., Prothero, J. D., Thornton, C. A., Pickard, K. M., Di Sabatino, A., Goggin, P. M., Pender, S. L. \& Macdonald, T. T. (2009) CC-10004 but not thalidomide or lenalidomide inhibits lamina propria mononuclear cell TNF-alpha and MMP-3 production in patients with inflammatory bowel disease. $J$ Crohns Colitis, 3(3), 175-82.

Gutschalk, C. M., Yanamandra, A. K., Linde, N., Meides, A., Depner, S. \& Mueller, M. M. (2013) GMCSF enhances tumor invasion by elevated MMP-2, -9, and -26 expression. Cancer Med, 2(2), 117-29.

Hamze, A. B., Wei, S., Bahudhanapati, H., Kota, S., Acharya, K. R. \& Brew, K. (2007) Constraining specificity in the $\mathrm{N}$-domain of tissue inhibitor of metalloproteinases-1; gelatinase-selective inhibitors. Protein Sci, 16(9), 1905-13. 
Hanai, H., Iida, T., Takeuchi, K., Watanabe, F., Maruyama, Y., Andoh, A., Tsujikawa, T., Fujiyama, Y., Mitsuyama, K., Sata, M., Yamada, M., Iwaoka, Y., Kanke, K., Hiraishi, H., Hirayama, K., Arai, H., Yoshii, S., Uchijima, M., Nagata, T. \& Koide, Y. (2006) Curcumin maintenance therapy for ulcerative colitis: randomized, multicenter, double-blind, placebo-controlled trial. Clin Gastroenterol Hepatol, 4(12), 1502-6.

Hanauer, S. B., Feagan, B. G., Lichtenstein, G. R., Mayer, L. F., Schreiber, S., Colombel, J. F., Rachmilewitz, D., Wolf, D. C., Olson, A., Bao, W. \& Rutgeerts, P. (2002) Maintenance infliximab for Crohn's disease: the ACCENT I randomised trial. Lancet, 359(9317), 1541-9.

Hanauer, S. B., Sandborn, W. J., Rutgeerts, P., Fedorak, R. N., Lukas, M., MacIntosh, D., Panaccione, R., Wolf, D. \& Pollack, P. (2006) Human anti-tumor necrosis factor monoclonal antibody (adalimumab) in Crohn's disease: the CLASSIC-I trial. Gastroenterology, 130(2), 323-33; quiz 591.

Hans, W., Scholmerich, J., Gross, V. \& Falk, W. (2000) The role of the resident intestinal flora in acute and chronic dextran sulfate sodium-induced colitis in mice. Eur J Gastroenterol Hepatol, 12(3), 26773.

Hayakawa, T., Yamashita, K., Ohuchi, E. \& Shinagawa, A. (1994) Cell growth-promoting activity of tissue inhibitor of metalloproteinases-2 (TIMP-2). J Cell Sci, 107 ( Pt 9), 2373-9.

Heimesaat, M. M., Dunay, I. R., Fuchs, D., Trautmann, D., Fischer, A., Kuhl, A. A., Loddenkemper, C., Batra, A., Siegmund, B., Krell, H. W., Bereswill, S. \& Liesenfeld, O. (2011a) Selective gelatinase blockage ameliorates acute DSS colitis. Eur J Microbiol Immunol (Bp), 1(3), 228-36.

Heimesaat, M. M., Dunay, I. R., Fuchs, D., Trautmann, D., Fischer, A., Kuhl, A. A., Loddenkemper, C., Siegmund, B., Batra, A., Bereswill, S. \& Liesenfeld, O. (2011b) The distinct roles of MMP-2 and MMP9 in acute DSS colitis. Eur J Microbiol Immunol (Bp), 1(4), 302-10.

Heimesaat, M. M., Heilmann, K., Kuhl, A. A., Erben, U., Ruhl, M., Fischer, A., Farndale, R. W., Bereswill, S., Gobel, U. B., Zeitz, M., Somasundaram, R. \& Freise, C. (2012) The synthetic hydroxyproline-containing collagen analogue (Gly-Pro-Hyp)10 ameliorates acute DSS colitis. Eur J Microbiol Immunol (Bp), 2(3), 192-200. 
Heljasvaara, R., Nyberg, P., Luostarinen, J., Parikka, M., Heikkila, P., Rehn, M., Sorsa, T., Salo, T. \& Pihlajaniemi, T. (2005) Generation of biologically active endostatin fragments from human collagen XVIII by distinct matrix metalloproteases. Exp Cell Res, 307(2), 292-304.

Heller, F., Florian, P., Bojarski, C., Richter, J., Christ, M., Hillenbrand, B., Mankertz, J., Gitter, A. H., Burgel, N., Fromm, M., Zeitz, M., Fuss, I., Strober, W. \& Schulzke, J. D. (2005) Interleukin-13 is the key effector Th2 cytokine in ulcerative colitis that affects epithelial tight junctions, apoptosis, and cell restitution. Gastroenterology, 129(2), 550-64.

Hernandez-Barrantes, S., Toth, M., Bernardo, M. M., Yurkova, M., Gervasi, D. C., Raz, Y., Sang, Q. A. \& Fridman, R. (2000) Binding of active (57 kDa) membrane type 1-matrix metalloproteinase (MT1MMP) to tissue inhibitor of metalloproteinase (TIMP)-2 regulates MT1-MMP processing and proMMP-2 activation. $J$ Biol Chem, 275(16), 12080-9.

Heuschkel, R. B., MacDonald, T. T., Monteleone, G., Bajaj-Elliott, M., Smith, J. A. \& Pender, S. L. (2000) Imbalance of stromelysin-1 and TIMP-1 in the mucosal lesions of children with inflammatory bowel disease. Gut, 47(1), 57-62.

Hibi, T., Ogata, H. \& Sakuraba, A. (2002) Animal models of inflammatory bowel disease. $J$ Gastroenterol, 37(6), 409-17.

Hocevar, B. J. \& Remzi, F. (2001) The ileal pouch anal anastomosis: past, present, and future. J Wound Ostomy Continence Nurs, 28(1), 32-6.

Holten-Andersen, M. N., Christensen, I. J., Nielsen, H. J., Stephens, R. W., Jensen, V., Nielsen, O. H., Sorensen, S., Overgaard, J., Lilja, H., Harris, A., Murphy, G. \& Brunner, N. (2002) Total levels of tissue inhibitor of metalloproteinases 1 in plasma yield high diagnostic sensitivity and specificity in patients with colon cancer. Clin Cancer Res, 8(1), 156-64.

Horiuchi, T., Mitoma, H., Harashima, S., Tsukamoto, H. \& Shimoda, T. (2010) Transmembrane TNFalpha: structure, function and interaction with anti-TNF agents. Rheumatology (Oxford), 49(7), 121528. 
Hu, J., Van den Steen, P. E., Sang, Q. X. \& Opdenakker, G. (2007) Matrix metalloproteinase inhibitors as therapy for inflammatory and vascular diseases. Nat Rev Drug Discov, 6(6), 480-98.

Huang, T. Y., Chu, H. C., Lin, Y. L., Lin, C. K., Hsieh, T. Y., Chang, W. K., Chao, Y. C. \& Liao, C. L. (2009) Minocycline attenuates experimental colitis in mice by blocking expression of inducible nitric oxide synthase and matrix metalloproteinases. Toxicol Appl Pharmacol, 237(1), 69-82.

Ishida, K., Takai, S., Murano, M., Nishikawa, T., Inoue, T., Murano, N., Inoue, N., Jin, D., Umegaki, E., Higuchi, K. \& Miyazaki, M. (2008) Role of chymase-dependent matrix metalloproteinase-9 activation in mice with dextran sodium sulfate-induced colitis. J Pharmacol Exp Ther, 324(2), 422-6.

Iskandar, H. N. \& Ciorba, M. A. (2012) Biomarkers in inflammatory bowel disease: current practices and recent advances. Transl Res, 159(4), 313-25.

Ito, A., Mukaiyama, A., Itoh, Y., Nagase, H., Thogersen, I. B., Enghild, J. J., Sasaguri, Y. \& Mori, Y. (1996) Degradation of interleukin 1beta by matrix metalloproteinases. J Biol Chem, 271(25), 14657-60.

Ito, A., Sato, T., Iga, T. \& Mori, Y. (1990) Tumor necrosis factor bifunctionally regulates matrix metalloproteinases and tissue inhibitor of metalloproteinases (TIMP) production by human fibroblasts. FEBS Lett, 269(1), 93-5.

Itoh, Y. \& Seiki, M. (2004) MT1-MMP: an enzyme with multidimensional regulation. Trends Biochem Sci, 29(6), 285-9.

Ivanov, II, Atarashi, K., Manel, N., Brodie, E. L., Shima, T., Karaoz, U., Wei, D., Goldfarb, K. C., Santee, C. A., Lynch, S. V., Tanoue, T., Imaoka, A., Itoh, K., Takeda, K., Umesaki, Y., Honda, K. \& Littman, D. R. (2009) Induction of intestinal Th17 cells by segmented filamentous bacteria. Cell, 139(3), 485-98.

Jimbo, K., Ohtsuka, Y., Kojima, Y., Hosoi, K., Ohbayashi, N., Ikuse, T., Aoyagi, Y., Fujii, T., Kudo, T. \& Shimizu, T. (2014) Increased expression of CXCR3 axis components and matrix metalloproteinase in pediatric inflammatory bowel disease patients. Pediatr Int, 56(6), 873-83. 
Jones-Hall, Y. L. \& Grisham, M. B. (2014) Immunopathological characterization of selected mouse models of inflammatory bowel disease: Comparison to human disease. Pathophysiology, 21(4), 267-88. Jostins, L., Ripke, S., Weersma, R. K., Duerr, R. H., McGovern, D. P., Hui, K. Y., Lee, J. C., Schumm, L. P., Sharma, Y., Anderson, C. A., Essers, J., Mitrovic, M., Ning, K., Cleynen, I., Theatre, E., Spain, S. L., Raychaudhuri, S., Goyette, P., Wei, Z., Abraham, C., Achkar, J. P., Ahmad, T., Amininejad, L., Ananthakrishnan, A. N., Andersen, V., Andrews, J. M., Baidoo, L., Balschun, T., Bampton, P. A., Bitton, A., Boucher, G., Brand, S., Büning, C., Cohain, A., Cichon, S., D’Amato, M., De Jong, D., Devaney, K. L., Dubinsky, M., Edwards, C., Ellinghaus, D., Ferguson, L. R., Franchimont, D., Fransen, K., Gearry, R., Georges, M., Gieger, C., Glas, J., Haritunians, T., Hart, A., Hawkey, C., Hedl, M., Hu, X., Karlsen, T. H., Kupcinskas, L., Kugathasan, S., Latiano, A., Laukens, D., Lawrance, I. C., Lees, C. W., Louis, E., Mahy, G., Mansfield, J., Morgan, A. R., Mowat, C., Newman, W., Palmieri, O., Ponsioen, C. Y., Potocnik, U., Prescott, N. J., Regueiro, M., Rotter, J. I., Russell, R. K., Sanderson, J. D., Sans, M., Satsangi, J., Schreiber, S., Simms, L. A., Sventoraityte, J., Targan, S. R., Taylor, K. D., Tremelling, M., Verspaget, H. W., De Vos, M., Wijmenga, C., Wilson, D. C., Winkelmann, J., Xavier, R. J., Zeissig, S., Zhang, B., Zhang, C. K., Zhao, H., Silverberg, M. S., Annese, V., Hakonarson, H., Brant, S. R., Radford-Smith, G., Mathew, C. G., Rioux, J. D., Schadt, E. E., et al (2012) Host-microbe interactions have shaped the genetic architecture of inflammatory bowel disease. Nature, 491(7422), 119-24.

Jung, K. K., Liu, X. W., Chirco, R., Fridman, R. \& Kim, H. R. (2006) Identification of CD63 as a tissue inhibitor of metalloproteinase-1 interacting cell surface protein. Embo j, 25(17), 3934-42.

Jurgens, M., Mahachie John, J. M., Cleynen, I., Schnitzler, F., Fidder, H., van Moerkercke, W., Ballet, V., Noman, M., Hoffman, I., van Assche, G., Rutgeerts, P. J., van Steen, K. \& Vermeire, S. (2011) Levels of C-reactive protein are associated with response to infliximab therapy in patients with Crohn's disease. Clin Gastroenterol Hepatol, 9(5), 421-7.e1.

Kaiser, T., Langhorst, J., Wittkowski, H., Becker, K., Friedrich, A. W., Rueffer, A., Dobos, G. J., Roth, J. \& Foell, D. (2007) Faecal S100A12 as a non-invasive marker distinguishing inflammatory bowel disease from irritable bowel syndrome. Gut, 56(12), 1706-13. 
Kallel, L., Feki, M., Sekri, W., Segheir, L., Fekih, M., Boubaker, J., Kaabachi, N. \& Filali, A. (2011) Prevalence and risk factors of hyperhomocysteinemia in Tunisian patients with Crohn's disease. $J$ Crohns Colitis, 5(2), 110-4.

Kamada, N., Seo, S. U., Chen, G. Y. \& Nunez, G. (2013) Role of the gut microbiota in immunity and inflammatory disease. Nat Rev Immunol, 13(5), 321-35.

Kane, S. V., Sandborn, W. J., Rufo, P. A., Zholudev, A., Boone, J., Lyerly, D., Camilleri, M. \& Hanauer, S. B. (2003) Fecal lactoferrin is a sensitive and specific marker in identifying intestinal inflammation. Am J Gastroenterol, 98(6), 1309-14.

Kang, K. H., Park, S. Y., Rho, S. B. \& Lee, J. H. (2008) Tissue inhibitor of metalloproteinases-3 interacts with angiotensin II type 2 receptor and additively inhibits angiogenesis. Cardiovasc Res, 79(1), 150-60.

Kaplan, G. G. (2015) The global burden of IBD: from 2015 to 2025. Nat Rev Gastroenterol Hepatol, 12(12), 720-7.

Kaplan, G. G., Seow, C. H., Ghosh, S., Molodecky, N., Rezaie, A., Moran, G. W., Proulx, M. C., Hubbard, J., MacLean, A., Buie, D. \& Panaccione, R. (2012) Decreasing colectomy rates for ulcerative colitis: a population-based time trend study. Am J Gastroenterol, 107(12), 1879-87.

Kapsoritakis, A. N., Kapsoritaki, A. I., Davidi, I. P., Lotis, V. D., Manolakis, A. C., Mylonis, P. I., Theodoridou, A. T., Germenis, A. E. \& Potamianos, S. P. (2008) Imbalance of tissue inhibitors of metalloproteinases (TIMP) - 1 and - 4 serum levels, in patients with inflammatory bowel disease. BMC Gastroenterol, 8, 55.

Karlsen, T. H. \& Boberg, K. M. (2013) Update on primary sclerosing cholangitis. J Hepatol, 59(3), 57182.

Kawabata, K., Murakami, A. \& Ohigashi, H. (2006a) Auraptene decreases the activity of matrix metalloproteinases in dextran sulfate sodium-induced ulcerative colitis in ICR mice. Biosci Biotechnol Biochem, 70(12), 3062-5. 
Kawabata, K., Murakami, A. \& Ohigashi, H. (2006b) Citrus auraptene targets translation of MMP-7 (matrilysin) via ERK1/2-dependent and mTOR-independent mechanism. FEBS Lett, 580(22), 5288-94. Khan, K. J., Dubinsky, M. C., Ford, A. C., Ullman, T. A., Talley, N. J. \& Moayyedi, P. (2011) Efficacy of immunosuppressive therapy for inflammatory bowel disease: a systematic review and meta-analysis. Am J Gastroenterol, 106(4), 630-42.

Kim, H. \& Koh, G. (2000) Lipopolysaccharide activates matrix metalloproteinase-2 in endothelial cells through an NF-kappaB-dependent pathway. Biochem Biophys Res Commun, 269(2), 401-5.

Kim, H. S., Shang, T., Chen, Z., Pflugfelder, S. C. \& Li, D. Q. (2004) TGF-beta1 stimulates production of gelatinase (MMP-9), collagenases (MMP-1, -13) and stromelysins (MMP-3, -10, -11) by human corneal epithelial cells. Exp Eye Res, 79(2), 263-74.

Kim, K. C. \& Lee, C. H. (2005) MAP kinase activation is required for the MMP-9 induction by TNFstimulation. Arch Pharm Res, 28(11), 1257-62.

Kirkegaard, T., Hansen, A., Bruun, E. \& Brynskov, J. (2004) Expression and localisation of matrix metalloproteinases and their natural inhibitors in fistulae of patients with Crohn's disease. Gut, 53(5), 701-9.

Kitani, A. \& Xu, L. (2008) Regulatory T cells and the induction of IL-17. Mucosal Immunol, 1 Suppl 1, S43-6.

Kjeldsen, L., Bainton, D. F., Sengelov, H. \& Borregaard, N. (1994) Identification of neutrophil gelatinase-associated lipocalin as a novel matrix protein of specific granules in human neutrophils. Blood, 83(3), 799-807.

Kobayashi, K., Arimura, Y., Goto, A., Okahara, S., Endo, T., Shinomura, Y. \& Imai, K. (2006) Therapeutic implications of the specific inhibition of causative matrix metalloproteinases in experimental colitis induced by dextran sulphate sodium. J Pathol, 209(3), 376-83.

Kobuch, J., Cui, H., Grunwald, B., Saftig, P., Knolle, P. A. \& Kruger, A. (2015) TIMP-1 signaling via CD63 triggers granulopoiesis and neutrophilia in mice. Haematologica, 100(8), 1005-13. 
Koelink, P. J., Overbeek, S. A., Braber, S., Morgan, M. E., Henricks, P. A., Abdul Roda, M., Verspaget, H. W., Wolfkamp, S. C., te Velde, A. A., Jones, C. W., Jackson, P. L., Blalock, J. E., Sparidans, R. W., Kruijtzer, J. A., Garssen, J., Folkerts, G. \& Kraneveld, A. D. (2014) Collagen degradation and neutrophilic infiltration: a vicious circle in inflammatory bowel disease. Gut, 63(4), 578-87.

Kofla-Dlubacz, A., Matusiewicz, M., Krzesiek, E., Noga, L. \& Iwanczak, B. (2014) Metalloproteinase3 and -9 as novel markers in the evaluation of ulcerative colitis activity in children. Adv Clin Exp Med, 23(1), 103-10.

Kolho, K. L., Sipponen, T., Valtonen, E. \& Savilahti, E. (2014) Fecal calprotectin, MMP-9, and human beta-defensin-2 levels in pediatric inflammatory bowel disease. Int J Colorectal Dis, 29(1), 43-50.

Koller, F. L., Dozier, E. A., Nam, K. T., Swee, M., Birkland, T. P., Parks, W. C. \& Fingleton, B. (2012) Lack of MMP10 exacerbates experimental colitis and promotes development of inflammationassociated colonic dysplasia. Lab Invest, 92(12), 1749-59.

Korkmaz, K. S., de Rooij, B. J., van Hoek, B., Janse, M., Coenraad, M. J., van der Reijden, J. J., Weersma, R. K., Porte, R. J., Voorneveld, P. W., Baranski, A. G. \& Verspaget, H. W. (2014) MMP-2 is a disease-modifying gene in primary sclerosing cholangitis. Liver Int, 34(2), 274-80.

Kruger, A., Kates, R. E. \& Edwards, D. R. (2010) Avoiding spam in the proteolytic internet: future strategies for anti-metastatic MMP inhibition. Biochim Biophys Acta, 1803(1), 95-102.

Kruidenier, L., MacDonald, T. T., Collins, J. E., Pender, S. L. \& Sanderson, I. R. (2006) Myofibroblast matrix metalloproteinases activate the neutrophil chemoattractant CXCL7 from intestinal epithelial cells. Gastroenterology, 130(1), 127-36.

Kucharzik, T., Walsh, S. V., Chen, J., Parkos, C. A. \& Nusrat, A. (2001) Neutrophil transmigration in inflammatory bowel disease is associated with differential expression of epithelial intercellular junction proteins. Am J Pathol, 159(6), 2001-9.

Kuchroo, V. K., Ohashi, P. S., Sartor, R. B. \& Vinuesa, C. G. (2012) Dysregulation of immune homeostasis in autoimmune diseases. Nat Med, 18(1), 42-7. 
Kuhn, R., Lohler, J., Rennick, D., Rajewsky, K. \& Muller, W. (1993) Interleukin-10-deficient mice develop chronic enterocolitis. Cell, 75(2), 263-74.

Kullberg, M. C., Andersen, J. F., Gorelick, P. L., Caspar, P., Suerbaum, S., Fox, J. G., Cheever, A. W., Jankovic, D. \& Sher, A. (2003) Induction of colitis by a CD4+ T cell clone specific for a bacterial epitope. Proc Natl Acad Sci U S A, 100(26), 15830-5.

Kundu, P., Ling, T. W., Korecka, A., Li, Y., D'Arienzo, R., Bunte, R. M., Berger, T., Arulampalam, V., Chambon, P., Mak, T. W., Wahli, W. \& Pettersson, S. (2014) Absence of intestinal PPARgamma aggravates acute infectious colitis in mice through a lipocalin-2-dependent pathway. PLoS Pathog, 10(1), e1003887.

Kurata, J. H., Kantor-Fish, S., Frankl, H., Godby, P. \& Vadheim, C. M. (1992) Crohn's disease among ethnic groups in a large health maintenance organization. Gastroenterology, 102(6), 1940-8.

Lai, W. C., Zhou, M., Shankavaram, U., Peng, G. \& Wahl, L. M. (2003) Differential regulation of lipopolysaccharide-induced monocyte matrix metalloproteinase (MMP)-1 and MMP-9 by p38 and extracellular signal-regulated kinase 1/2 mitogen-activated protein kinases. J Immunol, 170(12), 62449.

Lakatos, G., Sipos, F., Miheller, P., Hritz, I., Varga, M. Z., Juhasz, M., Molnar, B., Tulassay, Z. \& Herszenyi, L. (2011) The Behavior of Matrix Metalloproteinase-9 in Lymphocytic Colitis, Collagenous Colitis and Ulcerative Colitis. Pathol Oncol Res.

Lambert, E., Dasse, E., Haye, B. \& Petitfrere, E. (2004) TIMPs as multifacial proteins. Crit Rev Oncol Hematol, 49(3), 187-98.

Landy, J., Al-Hassi, H. O., McLaughlin, S. D., Knight, S. C., Ciclitira, P. J., Nicholls, R. J., Clark, S. K. \& Hart, A. L. (2012) Etiology of pouchitis. Inflamm Bowel Dis, 18(6), 1146-55.

Langers, A. M., Verspaget, H. W., Hommes, D. W. \& Sier, C. F. (2011) Single-nucleotide polymorphisms of matrix metalloproteinases and their inhibitors in gastrointestinal cancer. World $J$ Gastrointest Oncol, 3(6), 79-98. 
Lawrance, I. C., Fiocchi, C. \& Chakravarti, S. (2001) Ulcerative colitis and Crohn's disease: distinctive gene expression profiles and novel susceptibility candidate genes. Hum Mol Genet, 10(5), 445-56.

Lawrance, I. C., Wu, F., Leite, A. Z., Willis, J., West, G. A., Fiocchi, C. \& Chakravarti, S. (2003) A murine model of chronic inflammation-induced intestinal fibrosis down-regulated by antisense NFkappa B. Gastroenterology, 125(6), 1750-61.

Lee, J. Y., Woo, E. R. \& Kang, K. W. (2005a) Inhibition of lipopolysaccharide-inducible nitric oxide synthase expression by acteoside through blocking of AP-1 activation. J Ethnopharmacol, 97(3), 5616.

Lee, S., Jilani, S. M., Nikolova, G. V., Carpizo, D. \& Iruela-Arispe, M. L. (2005b) Processing of VEGFA by matrix metalloproteinases regulates bioavailability and vascular patterning in tumors. J Cell Biol, 169(4), 681-91.

Lees, C. W., Barrett, J. C., Parkes, M. \& Satsangi, J. (2011) New IBD genetics: common pathways with other diseases. Gut, 60(12), 1739-53.

Leon, A. J., Gomez, E., Garrote, J. A., Bernardo, D., Barrera, A., Marcos, J. L., Fernandez-Salazar, L., Velayos, B., Blanco-Quiros, A. \& Arranz, E. (2009) High levels of proinflammatory cytokines, but not markers of tissue injury, in unaffected intestinal areas from patients with IBD. Mediators Inflamm, 2009, 580450.

Li, C. K., Pender, S. L., Pickard, K. M., Chance, V., Holloway, J. A., Huett, A., Goncalves, N. S., Mudgett, J. S., Dougan, G., Frankel, G. \& MacDonald, T. T. (2004) Impaired immunity to intestinal bacterial infection in stromelysin-1 (matrix metalloproteinase-3)-deficient mice. J Immunol, 173(8), $5171-9$.

Linden, S. K., Sheng, Y. H., Every, A. L., Miles, K. M., Skoog, E. C., Florin, T. H., Sutton, P. \& McGuckin, M. A. (2009) MUC1 limits Helicobacter pylori infection both by steric hindrance and by acting as a releasable decoy. PLoS Pathog, 5(10), e1000617. 
Liu, H., Patel, N. R., Walter, L., Ingersoll, S., Sitaraman, S. V. \& Garg, P. (2013) Constitutive expression of MMP9 in intestinal epithelium worsens murine acute colitis and is associated with increased levels of proinflammatory cytokine Kc. Am J Physiol Gastrointest Liver Physiol, 304(9), G793-803.

Liu, J. Z., van Sommeren, S., Huang, H., Ng, S. C., Alberts, R., Takahashi, A., Ripke, S., Lee, J. C., Jostins, L., Shah, T., Abedian, S., Cheon, J. H., Cho, J., Daryani, N. E., Franke, L., Fuyuno, Y., Hart, A., Juyal, R. C., Juyal, G., Kim, W. H., Morris, A. P., Poustchi, H., Newman, W. G., Midha, V., Orchard, T. R., Vahedi, H., Sood, A., Sung, J. J., Malekzadeh, R., Westra, H. J., Yamazaki, K., Yang, S. K., Barrett, J. C., Franke, A., Alizadeh, B. Z., Parkes, M., B, K. T., Daly, M. J., Kubo, M., Anderson, C. A. \& Weersma, R. K. (2015) Association analyses identify 38 susceptibility loci for inflammatory bowel disease and highlight shared genetic risk across populations. Nat Genet, 47(9), 979-86.

Loftus, E. V., Jr. (2004) Clinical epidemiology of inflammatory bowel disease: Incidence, prevalence, and environmental influences. Gastroenterology, 126(6), 1504-17.

Lopez-Boado, Y. S., Wilson, C. L., Hooper, L. V., Gordon, J. I., Hultgren, S. J. \& Parks, W. C. (2000) Bacterial exposure induces and activates matrilysin in mucosal epithelial cells. J Cell Biol, 148(6), 130515.

Louis, E., Ribbens, C., Godon, A., Franchimont, D., De Groote, D., Hardy, N., Boniver, J., Belaiche, J. \& Malaise, M. (2000) Increased production of matrix metalloproteinase-3 and tissue inhibitor of metalloproteinase-1 by inflamed mucosa in inflammatory bowel disease. Clin Exp Immunol, 120(2), 241-6.

Ma, Y., Ohmen, J. D., Li, Z., Bentley, L. G., McElree, C., Pressman, S., Targan, S. R., Fischel-Ghodsian, N., Rotter, J. I. \& Yang, H. (1999) A genome-wide search identifies potential new susceptibility loci for Crohn's disease. Inflamm Bowel Dis, 5(4), 271-8.

Madisch, A., Hellmig, S., Schreiber, S., Bethke, B., Stolte, M. \& Miehlke, S. (2011) Allelic variation of the matrix metalloproteinase-9 gene is associated with collagenous colitis. Inflamm Bowel Dis, 17(11), 2295-8 
Mahler, M., Bristol, I. J., Leiter, E. H., Workman, A. E., Birkenmeier, E. H., Elson, C. O. \& Sundberg, J. P. (1998) Differential susceptibility of inbred mouse strains to dextran sulfate sodium-induced colitis. Am J Physiol, 274(3 Pt 1), G544-51.

Mahler, M. \& Leiter, E. H. (2002) Genetic and environmental context determines the course of colitis developing in IL-10-deficient mice. Inflamm Bowel Dis, 8(5), 347-55.

Makitalo, L., Kolho, K. L., Karikoski, R., Anthoni, H. \& Saarialho-Kere, U. (2010) Expression profiles of matrix metalloproteinases and their inhibitors in colonic inflammation related to pediatric inflammatory bowel disease. Scand J Gastroenterol, 45(7-8), 862-71.

Makitalo, L., Piekkala, M., Ashorn, M., Pakarinen, M., Koivusalo, A., Karikoski, R., Natunen, J., Saarialho-Kere, U., Rintala, R. \& Kolho, K. L. (2012a) Matrix metalloproteinases in the restorative proctocolectomy pouch of pediatric ulcerative colitis. World J Gastroenterol, 18(30), 4028-36.

Makitalo, L., Rintamaki, H., Tervahartiala, T., Sorsa, T. \& Kolho, K. L. (2012b) Serum MMPs 7-9 and their inhibitors during glucocorticoid and anti-TNF-alpha therapy in pediatric inflammatory bowel disease. Scand J Gastroenterol, 47(7), 785-94.

Makitalo, L., Sipponen, T., Karkkainen, P., Kolho, K. L. \& Saarialho-Kere, U. (2009) Changes in matrix metalloproteinase (MMP) and tissue inhibitors of metalloproteinases (TIMP) expression profile in Crohn's disease after immunosuppressive treatment correlate with histological score and calprotectin values. Int J Colorectal Dis, 24(10), 1157-67.

Manfredi, M. A., Zurakowski, D., Rufo, P. A., Walker, T. R., Fox, V. L. \& Moses, M. A. (2008) Increased incidence of urinary matrix metalloproteinases as predictors of disease in pediatric patients with inflammatory bowel disease. Inflamm Bowel Dis, 14(8), 1091-6.

Manolakis, A. C., Kapsoritakis, A. N., Georgoulias, P., Tzavara, C., Valotassiou, V., Kapsoritaki, A. \& Potamianos, S. P. (2010) Moderate performance of serum S100A12, in distinguishing inflammatory bowel disease from irritable bowel syndrome. BMC Gastroenterol, 10, 118.

Mao, J. W., He, X. M., Tang, H. Y. \& Wang, Y. D. (2012a) Protective role of metalloproteinase inhibitor (AE-941) on ulcerative colitis in rats. World J Gastroenterol, 18(47), 7063-9. 
Mao, J. W., Tang, H. Y., Tan, X. Y. \& Wang, Y. D. (2012b) Effect of Etiasa on the expression of matrix metalloproteinase-2 and tumor necrosis factor-alpha in a rat model of ulcerative colitis. Mol Med Rep, 6(5), 996-1000.

Marchant, D. J., Bellac, C. L., Moraes, T. J., Wadsworth, S. J., Dufour, A., Butler, G. S., Bilawchuk, L. M., Hendry, R. G., Robertson, A. G., Cheung, C. T., Ng, J., Ang, L., Luo, Z., Heilbron, K., Norris, M. J., Duan, W., Bucyk, T., Karpov, A., Devel, L., Georgiadis, D., Hegele, R. G., Luo, H., Granville, D. J., Dive, V., McManus, B. M. \& Overall, C. M. (2014) A new transcriptional role for matrix metalloproteinase-12 in antiviral immunity. Nat Med, 20(5), 493-502.

Marshall, D. C., Lyman, S. K., McCauley, S., Kovalenko, M., Spangler, R., Liu, C., Lee, M., O'Sullivan, C., Barry-Hamilton, V., Ghermazien, H., Mikels-Vigdal, A., Garcia, C. A., Jorgensen, B., Velayo, A. C., Wang, R., Adamkewicz, J. I. \& Smith, V. (2015) Selective Allosteric Inhibition of MMP9 Is Efficacious in Preclinical Models of Ulcerative Colitis and Colorectal Cancer. PLoS One, 10(5), e0127063.

Marshall, J. K., Thabane, M., Steinhart, A. H., Newman, J. R., Anand, A. \& Irvine, E. J. (2010) Rectal 5-aminosalicylic acid for induction of remission in ulcerative colitis. Cochrane Database Syst Rev(1), Cd004115.

Marshall, J. K., Thabane, M., Steinhart, A. H., Newman, J. R., Anand, A. \& Irvine, E. J. (2012) Rectal 5-aminosalicylic acid for maintenance of remission in ulcerative colitis. Cochrane Database Syst Rev, 11, Cd004118.

Martens, E., Leyssen, A., Van Aelst, I., Fiten, P., Piccard, H., Hu, J., Descamps, F. J., Van den Steen, P. E., Proost, P., Van Damme, J., Liuzzi, G. M., Riccio, P., Polverini, E. \& Opdenakker, G. (2007) A monoclonal antibody inhibits gelatinase B/MMP-9 by selective binding to part of the catalytic domain and not to the fibronectin or zinc binding domains. Biochim Biophys Acta, 1770(2), 178-86.

Martinesi, M., Ambrosini, S., Treves, C., Zuegel, U., Steinmeyer, A., Vito, A., Milla, M., Bonanomi, A. G. \& Stio, M. (2014) Role of vitamin D derivatives in intestinal tissue of patients with inflammatory bowel diseases. J Crohns Colitis, 8(9), 1062-71. 
Martinesi, M., Treves, C., Bonanomi, A. G., Milla, M., Bagnoli, S., Zuegel, U., Steinmeyer, A. \& Stio, M. (2010) Down-regulation of adhesion molecules and matrix metalloproteinases by ZK 156979 in inflammatory bowel diseases. Clin Immunol, 136(1), 51-60.

Masure, S., Proost, P., Van Damme, J. \& Opdenakker, G. (1991) Purification and identification of 91kDa neutrophil gelatinase. Release by the activating peptide interleukin-8. Eur J Biochem, 198(2), 3918.

Matsumoto, S., Okabe, Y., Setoyama, H., Takayama, K., Ohtsuka, J., Funahashi, H., Imaoka, A., Okada, Y. \& Umesaki, Y. (1998) Inflammatory bowel disease-like enteritis and caecitis in a senescence accelerated mouse P1/Yit strain. Gut, 43(1), 71-8.

Matsuno, K., Adachi, Y., Yamamoto, H., Goto, A., Arimura, Y., Endo, T., Itoh, F. \& Imai, K. (2003) The expression of matrix metalloproteinase matrilysin indicates the degree of inflammation in ulcerative colitis. J Gastroenterol, 38(4), 348-54.

McKaig, B. C., McWilliams, D., Watson, S. A. \& Mahida, Y. R. (2003) Expression and regulation of tissue inhibitor of metalloproteinase-1 and matrix metalloproteinases by intestinal myofibroblasts in inflammatory bowel disease. Am J Pathol, 162(4), 1355-60.

Medina, C., Santana, A., Paz, M. C., Diaz-Gonzalez, F., Farre, E., Salas, A., Radomski, M. W. \& Quintero, E. (2006a) Matrix metalloproteinase-9 modulates intestinal injury in rats with transmural colitis. J Leukoc Biol, 79(5), 954-62.

Medina, C., Santana, A., Paz-Cabrera, M. C., Parra-Blanco, A., Nicolas, D., Gimeno-Garcia, A. Z. \& Quintero, E. (2006b) Increased activity and expression of gelatinases in ischemic colitis. Dig Dis Sci, 51(12), 2393-9.

Medina, C., Santos-Martinez, M. J., Santana, A., Paz-Cabrera, M. C., Johnston, M. J., Mourelle, M., Salas, A. \& Guarner, F. (2011) Transforming growth factor-beta type 1 receptor (ALK5) and Smad proteins mediate TIMP-1 and collagen synthesis in experimental intestinal fibrosis. J Pathol, 224(4), 461-72. 
Medina, C., Videla, S., Radomski, A., Radomski, M. W., Antolin, M., Guarner, F., Vilaseca, J., Salas, A. \& Malagelada, J. R. (2003) Increased activity and expression of matrix metalloproteinase-9 in a rat model of distal colitis. Am J Physiol Gastrointest Liver Physiol, 284(1), G116-22.

Meijer, M. J., Mieremet-Ooms, M. A., van der Zon, A. M., van Duijn, W., van Hogezand, R. A., Sier, C. F., Hommes, D. W., Lamers, C. B. \& Verspaget, H. W. (2007a) Increased mucosal matrix metalloproteinase-1, $-2,-3$ and -9 activity in patients with inflammatory bowel disease and the relation with Crohn's disease phenotype. Dig Liver Dis, 39(8), 733-9.

Meijer, M. J., Mieremet-Ooms, M. A., van Duijn, W., van der Zon, A. M., Hanemaaijer, R., Verheijen, J. H., van Hogezand, R. A., Lamers, C. B. \& Verspaget, H. W. (2007b) Effect of the anti-tumor necrosis factor-alpha antibody infliximab on the ex vivo mucosal matrix metalloproteinase-proteolytic phenotype in inflammatory bowel disease. Inflamm Bowel Dis, 13(2), 200-10.

Meijer, M. J., Mieremet-Ooms, M. A., van Hogezand, R. A., Lamers, C. B., Hommes, D. W. \& Verspaget, H. W. (2007c) Role of matrix metalloproteinase, tissue inhibitor of metalloproteinase and tumor necrosis factor-alpha single nucleotide gene polymorphisms in inflammatory bowel disease. World J Gastroenterol, 13(21), 2960-6.

Melgar, S., Karlsson, A. \& Michaelsson, E. (2005) Acute colitis induced by dextran sulfate sodium progresses to chronicity in $\mathrm{C} 57 \mathrm{BL} / 6$ but not in $\mathrm{BALB} / \mathrm{c}$ mice: correlation between symptoms and inflammation. Am J Physiol Gastrointest Liver Physiol, 288(6), G1328-38.

Mendoza, J. L. \& Abreu, M. T. (2009) Biological markers in inflammatory bowel disease: practical consideration for clinicians. Gastroenterol Clin Biol, 33 Suppl 3, S158-73.

Middleton, E., Jr. (1988) Some biological properties of plant flavonoids. Ann Allergy, 61(6 Pt 2), 53-7.

Miller, D. H., Khan, O. A., Sheremata, W. A., Blumhardt, L. D., Rice, G. P., Libonati, M. A., WillmerHulme, A. J., Dalton, C. M., Miszkiel, K. A. \& O'Connor, P. W. (2003) A controlled trial of natalizumab for relapsing multiple sclerosis. N Engl J Med, 348(1), 15-23. 
Mitoma, H., Horiuchi, T., Hatta, N., Tsukamoto, H., Harashima, S., Kikuchi, Y., Otsuka, J., Okamura, S., Fujita, S. \& Harada, M. (2005) Infliximab induces potent anti-inflammatory responses by outsideto-inside signals through transmembrane TNF-alpha. Gastroenterology, 128(2), 376-92.

Mohamadzadeh, M., Pfeiler, E. A., Brown, J. B., Zadeh, M., Gramarossa, M., Managlia, E., Bere, P., Sarraj, B., Khan, M. W., Pakanati, K. C., Ansari, M. J., O'Flaherty, S., Barrett, T. \& Klaenhammer, T. R. (2011) Regulation of induced colonic inflammation by Lactobacillus acidophilus deficient in lipoteichoic acid. Proc Natl Acad Sci U S A, 108 Suppl 1, 4623-30.

Molodecky, N. A., Soon, I. S., Rabi, D. M., Ghali, W. A., Ferris, M., Chernoff, G., Benchimol, E. I., Panaccione, R., Ghosh, S., Barkema, H. W. \& Kaplan, G. G. (2012) Increasing incidence and prevalence of the inflammatory bowel diseases with time, based on systematic review. Gastroenterology, 142(1), 46-54.e42; quiz e30.

Monteleone, G., Caruso, R., Fina, D., Peluso, I., Gioia, V., Stolfi, C., Fantini, M. C., Caprioli, F., Tersigni, R., Alessandroni, L., MacDonald, T. T. \& Pallone, F. (2006) Control of matrix metalloproteinase production in human intestinal fibroblasts by interleukin 21. Gut, 55(12), 1774-80.

Monteleone, I., Federici, M., Sarra, M., Franze, E., Casagrande, V., Zorzi, F., Cavalera, M., Rizzo, A., Lauro, R., Pallone, F., MacDonald, T. T. \& Monteleone, G. (2012) Tissue inhibitor of metalloproteinase3 regulates inflammation in human and mouse intestine. Gastroenterology, 143(5), 1277-87.e1-4.

Moore, C. S. \& Crocker, S. J. (2012) An alternate perspective on the roles of TIMPs and MMPs in pathology. Am J Pathol, 180(1), 12-6.

Morgan, A. R., Han, D. Y., Lam, W. J., Triggs, C. M., Fraser, A. G., Barclay, M., Gearry, R. B., Meisner, S., Stokkers, P., Boeckxstaens, G. E. \& Ferguson, L. R. (2011) Genetic variations in matrix metalloproteinases may be associated with increased risk of ulcerative colitis. Hum Immunol, 72(11), 1117-27.

Morgan, M. E., Zheng, B., Koelink, P. J., van de Kant, H. J., Haazen, L. C., van Roest, M., Garssen, J., Folkerts, G. \& Kraneveld, A. D. (2013) New perspective on dextran sodium sulfate colitis: antigenspecific T cell development during intestinal inflammation. PLoS One, 8(7), e69936. 
Morgan, X. C., Tickle, T. L., Sokol, H., Gevers, D., Devaney, K. L., Ward, D. V., Reyes, J. A., Shah, S. A., LeLeiko, N., Snapper, S. B., Bousvaros, A., Korzenik, J., Sands, B. E., Xavier, R. J. \& Huttenhower, C. (2012) Dysfunction of the intestinal microbiome in inflammatory bowel disease and treatment. Genome Biol, 13(9), R79.

Morgenstern, I., Raijmakers, M. T., Peters, W. H., Hoensch, H. \& Kirch, W. (2003) Homocysteine, cysteine, and glutathione in human colonic mucosa: elevated levels of homocysteine in patients with inflammatory bowel disease. Dig Dis Sci, 48(10), 2083-90.

Morgunova, E., Tuuttila, A., Bergmann, U. \& Tryggvason, K. (2002) Structural insight into the complex formation of latent matrix metalloproteinase 2 with tissue inhibitor of metalloproteinase 2. Proc Natl Acad Sci U S A, 99(11), 7414-9.

Morrison, C. J., Butler, G. S., Rodriguez, D. \& Overall, C. M. (2009) Matrix metalloproteinase proteomics: substrates, targets, and therapy. Curr Opin Cell Biol, 21(5), 645-53.

Mouli, V. P. \& Ananthakrishnan, A. N. (2014) Review article: vitamin D and inflammatory bowel diseases. Aliment Pharmacol Ther, 39(2), 125-36.

Munoz, M., Heimesaat, M. M., Danker, K., Struck, D., Lohmann, U., Plickert, R., Bereswill, S., Fischer, A., Dunay, I. R., Wolk, K., Loddenkemper, C., Krell, H. W., Libert, C., Lund, L. R., Frey, O., Holscher, C., Iwakura, Y., Ghilardi, N., Ouyang, W., Kamradt, T., Sabat, R. \& Liesenfeld, O. (2009) Interleukin (IL)-23 mediates Toxoplasma gondii-induced immunopathology in the gut via matrixmetalloproteinase2 and IL-22 but independent of IL-17. J Exp Med, 206(13), 3047-59.

Nagase, H., London, I. C., Murphy, G., Cambridge University, C. R. U. C. R. I., Li ka Shing Centre \& gm290@cam.ac.uk (2008) Tailoring TIMPs for Selective Metalloproteinase Inhibition, in Dylan Edwards, G. H.-H., Francesco Blasi, Bonnie F. Sloane (ed), The Cancer DegradomeSpringer New York, 787-810.

Nagase, H., Visse, R. \& Murphy, G. (2006) Structure and function of matrix metalloproteinases and TIMPs. Cardiovasc Res, 69(3), 562-73. 
Naito, Y., Takagi, T., Kuroda, M., Katada, K., Ichikawa, H., Kokura, S., Yoshida, N., Okanoue, T. \& Yoshikawa, T. (2004) An orally active matrix metalloproteinase inhibitor, ONO-4817, reduces dextran sulfate sodium-induced colitis in mice. Inflamm Res, 53(9), 462-8.

Nee, L. E., McMorrow, T., Campbell, E., Slattery, C. \& Ryan, M. P. (2004) TNF-alpha and IL-1betamediated regulation of MMP-9 and TIMP-1 in renal proximal tubular cells. Kidney Int, 66(4), 1376-86.

Nell, S., Suerbaum, S. \& Josenhans, C. (2010) The impact of the microbiota on the pathogenesis of IBD: lessons from mouse infection models. Nat Rev Microbiol, 8(8), 564-77.

Nielsen, O. H., Coskun, M., Steenholdt, C. \& Rogler, G. (2015) The role and advances of immunomodulator therapy for inflammatory bowel disease. Expert Rev Gastroenterol Hepatol, 9(2), 177-89.

Nielsen, O. H., Gionchetti, P., Ainsworth, M., Vainer, B., Campieri, M., Borregaard, N. \& Kjeldsen, L. (1999) Rectal dialysate and fecal concentrations of neutrophil gelatinase-associated lipocalin, interleukin-8, and tumor necrosis factor-alpha in ulcerative colitis. Am J Gastroenterol, 94(10), 2923-8. Nighot, P., Al-Sadi, R., Rawat, M., Guo, S., Watterson, D. M. \& Ma, T. (2015) Matrix metalloproteinase 9-induced increase in intestinal epithelial tight junction permeability contributes to the severity of experimental DSS colitis. Am J Physiol Gastrointest Liver Physiol, 309(12), G988-97.

Nilsson, U. W. \& Dabrosin, C. (2006) Estradiol and tamoxifen regulate endostatin generation via matrix metalloproteinase activity in breast cancer in vivo. Cancer Res, 66(9), 4789-94.

Noble, C. L., Abbas, A. R., Cornelius, J., Lees, C. W., Ho, G. T., Toy, K., Modrusan, Z., Pal, N., Zhong, F., Chalasani, S., Clark, H., Arnott, I. D., Penman, I. D., Satsangi, J. \& Diehl, L. (2008) Regional variation in gene expression in the healthy colon is dysregulated in ulcerative colitis. Gut, 57(10), 1398405.

Noble, C. L., Abbas, A. R., Lees, C. W., Cornelius, J., Toy, K., Modrusan, Z., Clark, H. F., Arnott, I. D., Penman, I. D., Satsangi, J. \& Diehl, L. (2010) Characterization of intestinal gene expression profiles in Crohn's disease by genome-wide microarray analysis. Inflamm Bowel Dis, 16(10), 1717-28. 
Norman, J. M., Handley, S. A., Baldridge, M. T., Droit, L., Liu, C. Y., Keller, B. C., Kambal, A., Monaco, C. L., Zhao, G., Fleshner, P., Stappenbeck, T. S., McGovern, D. P., Keshavarzian, A., Mutlu, E. A., Sauk, J., Gevers, D., Xavier, R. J., Wang, D., Parkes, M. \& Virgin, H. W. (2015) Disease-specific alterations in the enteric virome in inflammatory bowel disease. Cell, 160(3), 447-60.

O'Shea, N. R. \& Smith, A. M. (2014) Matrix metalloproteases role in bowel inflammation and inflammatory bowel disease: an up to date review. Inflamm Bowel Dis, 20(12), 2379-93.

O'Sullivan, S., Gilmer, J. F. \& Medina, C. (2015) Matrix metalloproteinases in inflammatory bowel disease: an update. Mediators Inflamm, 2015, 964131.

Odabasoglu, F., Halici, Z., Aygun, H., Halici, M., Atalay, F., Cakir, A., Cadirci, E., Bayir, Y. \& Suleyman, H. (2011) alpha-Lipoic acid has anti-inflammatory and anti-oxidative properties: an experimental study in rats with carrageenan-induced acute and cotton pellet-induced chronic inflammations. Br J Nutr, 105(1), 31-43.

Ogata, Y., Itoh, Y. \& Nagase, H. (1995) Steps involved in activation of the pro-matrix metalloproteinase 9 (progelatinase B)-tissue inhibitor of metalloproteinases-1 complex by 4-aminophenylmercuric acetate and proteinases. J Biol Chem, 270(31), 18506-11.

Ohkawara, T., Nishihira, J., Takeda, H., Hige, S., Kato, M., Sugiyama, T., Iwanaga, T., Nakamura, H., Mizue, Y. \& Asaka, M. (2002) Amelioration of dextran sulfate sodium-induced colitis by antimacrophage migration inhibitory factor antibody in mice. Gastroenterology, 123(1), 256-70.

Oikonomou, K. A., Kapsoritakis, A. N., Theodoridou, C., Karangelis, D., Germenis, A., Stefanidis, I. \& Potamianos, S. P. (2012) Neutrophil gelatinase-associated lipocalin (NGAL) in inflammatory bowel disease: association with pathophysiology of inflammation, established markers, and disease activity. $J$ Gastroenterol, 47(5), 519-30.

Okayasu, I., Hatakeyama, S., Yamada, M., Ohkusa, T., Inagaki, Y. \& Nakaya, R. (1990) A novel method in the induction of reliable experimental acute and chronic ulcerative colitis in mice. Gastroenterology, 98(3), 694-702. 
Oliveira, L. G., Cunha, A. L., Duarte, A. C., Castanon, M. C., Chebli, J. M. \& Aguiar, J. A. (2014) Positive correlation between disease activity index and matrix metalloproteinases activity in a rat model of colitis. Arq Gastroenterol, 51(2), 107-12.

Opdenakker, G., Masure, S., Grillet, B. \& Van Damme, J. (1991) Cytokine-mediated regulation of human leukocyte gelatinases and role in arthritis. Lymphokine Cytokine Res, 10(4), 317-24.

Opdenakker, G. \& Van Damme, J. (1994) Cytokine-regulated proteases in autoimmune diseases. Immunol Today, 15(3), 103-7.

Opdenakker, G. \& Van Damme, J. (2011) Probing cytokines, chemokines and matrix metalloproteinases towards better immunotherapies of multiple sclerosis. Cytokine Growth Factor Rev, 22(5-6), 359-65.

Opdenakker, G., Van den Steen, P. E., Dubois, B., Nelissen, I., Van Coillie, E., Masure, S., Proost, P. \& Van Damme, J. (2001a) Gelatinase B functions as regulator and effector in leukocyte biology. J Leukoc Biol, 69(6), 851-9.

Opdenakker, G., Van den Steen, P. E. \& Van Damme, J. (2001b) Gelatinase B: a tuner and amplifier of immune functions. Trends Immunol, 22(10), 571-9.

Overall, C. M. \& Kleifeld, O. (2006) Tumour microenvironment - opinion: validating matrix metalloproteinases as drug targets and anti-targets for cancer therapy. Nat Rev Cancer, 6(3), 227-39.

Overall, C. M. \& Lopez-Otin, C. (2002) Strategies for MMP inhibition in cancer: innovations for the post-trial era. Nat Rev Cancer, 2(9), 657-72.

Paemen, L., Martens, E., Masure, S. \& Opdenakker, G. (1995) Monoclonal antibodies specific for natural human neutrophil gelatinase B used for affinity purification, quantitation by two-site ELISA and inhibition of enzymatic activity. Eur J Biochem, 234(3), 759-65.

Park, D. K. \& Park, H. J. (2013) Ethanol extract of Cordyceps militaris grown on germinated soybeans attenuates dextran-sodium-sulfate- (DSS-) induced colitis by suppressing the expression of matrix metalloproteinases and inflammatory mediators. Biomed Res Int, 2013, 102918. 
Parronchi, P., Romagnani, P., Annunziato, F., Sampognaro, S., Becchio, A., Giannarini, L., Maggi, E., Pupilli, C., Tonelli, F. \& Romagnani, S. (1997) Type 1 T-helper cell predominance and interleukin-12 expression in the gut of patients with Crohn's disease. Am J Pathol, 150(3), 823-32.

Pedersen, G., Saermark, T., Kirkegaard, T. \& Brynskov, J. (2009) Spontaneous and cytokine induced expression and activity of matrix metalloproteinases in human colonic epithelium. Clin Exp Immunol, $155(2), 257-65$.

Pender, S. L., Croucher, P. J., Mascheretti, S., Prothero, J. D., Fisher, S. A., MacDonald, T. T., Schreiber, S. \& Ye, S. (2004) Transmission disequilibrium test of stromelysin-1 gene variation in relation to Crohn's disease. J Med Genet, 41(9), e112.

Pender, S. L., Fell, J. M., Chamow, S. M., Ashkenazi, A. \& MacDonald, T. T. (1998) A p55 TNF receptor immunoadhesin prevents $\mathrm{T}$ cell-mediated intestinal injury by inhibiting matrix metalloproteinase production. J Immunol, 160(8), 4098-103.

Pender, S. L., Li, C. K., Di Sabatino, A., MacDonald, T. T. \& Buckley, M. G. (2006) Role of macrophage metalloelastase in gut inflammation. Ann N Y Acad Sci, 1072, 386-8.

Pender, S. L., Salmela, M. T., Monteleone, G., Schnapp, D., McKenzie, C., Spencer, J., Fong, S., Saarialho-Kere, U. \& MacDonald, T. T. (2000) Ligation of alpha4ss1 integrin on human intestinal mucosal mesenchymal cells selectively Up-regulates membrane type-1 matrix metalloproteinase and confers a migratory phenotype. Am J Pathol, 157(6), 1955-62.

Pender, S. L., Tickle, S. P., Docherty, A. J., Howie, D., Wathen, N. C. \& MacDonald, T. T. (1997) A major role for matrix metalloproteinases in T cell injury in the gut. J Immunol, 158(4), 1582-90.

Perse, M. \& Cerar, A. (2012) Dextran sodium sulphate colitis mouse model: traps and tricks. J Biomed Biotechnol, 2012, 718617.

Peyrin-Biroulet, L., Rodriguez-Gueant, R. M., Chamaillard, M., Desreumaux, P., Xia, B., Bronowicki, J. P., Bigard, M. A. \& Gueant, J. L. (2007) Vascular and cellular stress in inflammatory bowel disease: revisiting the role of homocysteine. Am J Gastroenterol, 102(5), 1108-15. 
Piccard, H., Van den Steen, P. E. \& Opdenakker, G. (2007) Hemopexin domains as multifunctional liganding modules in matrix metalloproteinases and other proteins. J Leukoc Biol, 81(4), 870-92.

Piekkala, M., Hagstrom, J., Tanskanen, M., Rintala, R., Haglund, C. \& Kolho, K. L. (2013) Low trypsinogen-1 expression in pediatric ulcerative colitis patients who undergo surgery. World $J$ Gastroenterol, 19(21), 3272-80.

Pirila, E., Ramamurthy, N. S., Sorsa, T., Salo, T., Hietanen, J. \& Maisi, P. (2003) Gelatinase A (MMP2), collagenase-2 (MMP-8), and laminin-5 gamma2-chain expression in murine inflammatory bowel disease (ulcerative colitis). Dig Dis Sci, 48(1), 93-8.

Pope, J. L., Bhat, A. A., Sharma, A., Ahmad, R., Krishnan, M., Washington, M. K., Beauchamp, R. D., Singh, A. B. \& Dhawan, P. (2014) Claudin-1 regulates intestinal epithelial homeostasis through the modulation of Notch-signalling. Gut, 63(4), 622-34.

Powell, W. C., Fingleton, B., Wilson, C. L., Boothby, M. \& Matrisian, L. M. (1999) The metalloproteinase matrilysin proteolytically generates active soluble Fas ligand and potentiates epithelial cell apoptosis. Curr Biol, 9(24), 1441-7.

Powrie, F., Leach, M. W., Mauze, S., Caddle, L. B. \& Coffman, R. L. (1993) Phenotypically distinct subsets of CD4+ T cells induce or protect from chronic intestinal inflammation in C. B-17 scid mice. Int Immunol, 5(11), 1461-71.

Present, D. H., Rutgeerts, P., Targan, S., Hanauer, S. B., Mayer, L., van Hogezand, R. A., Podolsky, D. K., Sands, B. E., Braakman, T., DeWoody, K. L., Schaible, T. F. \& van Deventer, S. J. H. (1999) Infliximab for the treatment of fistulas in patients with Crohn's disease. New England Journal of Medicine, 340(18), 1398-1405.

Pruteanu, M. \& Shanahan, F. (2013) Digestion of epithelial tight junction proteins by the commensal Clostridium perfringens. Am J Physiol Gastrointest Liver Physiol, 305(10), G740-8.

Puthenedam, M., Wu, F., Shetye, A., Michaels, A., Rhee, K. J. \& Kwon, J. H. (2011) Matrilysin-1 (MMP7) cleaves galectin-3 and inhibits wound healing in intestinal epithelial cells. Inflamm Bowel Dis, 17(1), 260-7. 
Qi, J. H., Ebrahem, Q., Moore, N., Murphy, G., Claesson-Welsh, L., Bond, M., Baker, A. \& AnandApte, B. (2003) A novel function for tissue inhibitor of metalloproteinases-3 (TIMP3): inhibition of angiogenesis by blockage of VEGF binding to VEGF receptor-2. Nat Med, 9(4), 407-15.

Rath, T., Roderfeld, M., Graf, J., Wagner, S., Vehr, A. K., Dietrich, C., Geier, A. \& Roeb, E. (2006) Enhanced expression of MMP-7 and MMP-13 in inflammatory bowel disease: a precancerous potential? Inflamm Bowel Dis, 12(11), 1025-35.

Rath, T., Roderfeld, M., Halwe, J. M., Tschuschner, A., Roeb, E. \& Graf, J. (2010) Cellular sources of MMP-7, MMP-13 and MMP-28 in ulcerative colitis. Scand J Gastroenterol, 45(10), 1186-96.

Ravi, A., Garg, P. \& Sitaraman, S. V. (2007) Matrix metalloproteinases in inflammatory bowel disease: boon or a bane? Inflamm Bowel Dis, 13(1), 97-107.

Redondo-Munoz, J., Ugarte-Berzal, E., Garcia-Marco, J. A., del Cerro, M. H., Van den Steen, P. E., Opdenakker, G., Terol, M. J. \& Garcia-Pardo, A. (2008) Alpha4beta1 integrin and 190-kDa CD44v constitute a cell surface docking complex for gelatinase B/MMP-9 in chronic leukemic but not in normal B cells. Blood, 112(1), 169-78.

Redondo-Munoz, J., Ugarte-Berzal, E., Terol, M. J., Van den Steen, P. E., Hernandez del Cerro, M., Roderfeld, M., Roeb, E., Opdenakker, G., Garcia-Marco, J. A. \& Garcia-Pardo, A. (2010) Matrix metalloproteinase-9 promotes chronic lymphocytic leukemia b cell survival through its hemopexin domain. Cancer Cell, 17(2), 160-72.

Reinisch, W., Sandborn, W. J., Hommes, D. W., D'Haens, G., Hanauer, S., Schreiber, S., Panaccione, R., Fedorak, R. N., Tighe, M. B., Huang, B., Kampman, W., Lazar, A. \& Thakkar, R. (2011) Adalimumab for induction of clinical remission in moderately to severely active ulcerative colitis: results of a randomised controlled trial. Gut, 60(6), 780-7.

Rhee, J. W., Lee, K. W., Sohn, W. J., Lee, Y., Jeon, O. H., Kwon, H. J. \& Kim, D. S. (2007) Regulation of matrix metalloproteinase-9 gene expression and cell migration by NF-kappa B in response to CpGoligodeoxynucleotides in RAW 264.7 cells. Mol Immunol, 44(6), 1393-400. 
Rieder, F., Brenmoehl, J., Leeb, S., Scholmerich, J. \& Rogler, G. (2007) Wound healing and fibrosis in intestinal disease. Gut, 56(1), 130-9.

Rieder, F., de Bruyn, J. R., Pham, B. T., Katsanos, K., Annese, V., Higgins, P. D., Magro, F. \& Dotan, I. (2014) Results of the 4th scientific workshop of the ECCO (Group II): markers of intestinal fibrosis in inflammatory bowel disease. J Crohns Colitis, 8(10), 1166-78.

Rieder, F. \& Fiocchi, C. (2013) Mechanisms of tissue remodeling in inflammatory bowel disease. Dig Dis, 31(2), 186-93.

Rieder, F., Kessler, S., Sans, M. \& Fiocchi, C. (2012) Animal models of intestinal fibrosis: new tools for the understanding of pathogenesis and therapy of human disease. Am J Physiol Gastrointest Liver Physiol, 303(7), G786-801.

Rioux, J. D., Silverberg, M. S., Daly, M. J., Steinhart, A. H., McLeod, R. S., Griffiths, A. M., Green, T., Brettin, T. S., Stone, V., Bull, S. B., Bitton, A., Williams, C. N., Greenberg, G. R., Cohen, Z., Lander, E. S., Hudson, T. J. \& Siminovitch, K. A. (2000) Genomewide search in Canadian families with inflammatory bowel disease reveals two novel susceptibility loci. Am J Hum Genet, 66(6), 1863-70.

Rivera-Nieves, J., Bamias, G., Vidrich, A., Marini, M., Pizarro, T. T., McDuffie, M. J., Moskaluk, C. A., Cohn, S. M. \& Cominelli, F. (2003) Emergence of perianal fistulizing disease in the SAMP1/YitFc mouse, a spontaneous model of chronic ileitis. Gastroenterology, 124(4), 972-82.

Rodrigues, D. M., Sousa, A. J., Hawley, S. P., Vong, L., Gareau, M. G., Kumar, S. A., Johnson-Henry, K. C. \& Sherman, P. M. (2012) Matrix metalloproteinase 9 contributes to gut microbe homeostasis in a model of infectious colitis. BMC Microbiol, 12, 105.

Rodriguez, D., Morrison, C. J. \& Overall, C. M. (2010) Matrix metalloproteinases: what do they not do? New substrates and biological roles identified by murine models and proteomics. Biochim Biophys Acta, 1803(1), 39-54.

Roseth, A. G., Fagerhol, M. K., Aadland, E. \& Schjonsby, H. (1992) Assessment of the neutrophil dominating protein calprotectin in feces. A methodologic study. Scand J Gastroenterol, 27(9), 793-8. 
Round, J. L., Lee, S. M., Li, J., Tran, G., Jabri, B., Chatila, T. A. \& Mazmanian, S. K. (2011) The Tolllike receptor 2 pathway establishes colonization by a commensal of the human microbiota. Science, 332(6032), 974-7.

Round, J. L. \& Mazmanian, S. K. (2009) The gut microbiota shapes intestinal immune responses during health and disease. Nat Rev Immunol, 9(5), 313-23.

Ruehl, M., Muche, M., Freise, C., Erben, U., Neumann, U., Schuppan, D., Popov, Y., Dieterich, W., Zeitz, M., Farndale, R. W. \& Somasundaram, R. (2011) Hydroxyproline-containing collagen analogs trigger the release and activation of collagen-sequestered proMMP-2 by competition with prodomainderived peptide P33-42. Fibrogenesis Tissue Repair, 4(1), 1.

Russel, M. G., Pastoor, C. J., Janssen, K. M., van Deursen, C. T., Muris, J. W., van Wijlick, E. H. \& Stockbrugger, R. W. (1997) Familial aggregation of inflammatory bowel disease: a population-based study in South Limburg, The Netherlands. The South Limburg IBD Study Group. Scand J Gastroenterol Suppl, 223, 88-91.

Rutgeerts, P., Goboes, K., Peeters, M., Hiele, M., Penninckx, F., Aerts, R., Kerremans, R. \& Vantrappen, G. (1991) Effect of faecal stream diversion on recurrence of Crohn's disease in the neoterminal ileum. Lancet, 338(8770), 771-4.

Rutgeerts, P., Sandborn, W. J., Feagan, B. G., Reinisch, W., Olson, A., Johanns, J., Travers, S., Rachmilewitz, D., Hanauer, S. B., Lichtenstein, G. R., de Villiers, W. J., Present, D., Sands, B. E. \& Colombel, J. F. (2005) Infliximab for induction and maintenance therapy for ulcerative colitis. $N$ Engl J Med, 353(23), 2462-76.

Saarialho-Kere, U. K., Vaalamo, M., Puolakkainen, P., Airola, K., Parks, W. C. \& KarjalainenLindsberg, M. L. (1996) Enhanced expression of matrilysin, collagenase, and stromelysin-1 in gastrointestinal ulcers. Am J Pathol, 148(2), 519-26.

Saibeni, S., Bollani, S., Losco, A., Michielan, A., Sostegni, R., Devani, M., Lupinacci, G., Pirola, L., Cucino, C., Meucci, G., Basilisco, G., D'Inca, R. \& Bruno, S. (2012) The use of methotrexate for treatment of inflammatory bowel disease in clinical practice. Dig Liver Dis, 44(2), 123-7. 
Salmela, M. T., MacDonald, T. T., Black, D., Irvine, B., Zhuma, T., Saarialho-Kere, U. \& Pender, S. L. (2002) Upregulation of matrix metalloproteinases in a model of T cell mediated tissue injury in the gut: analysis by gene array and in situ hybridisation. Gut, 51(4), 540-7.

Salmela, M. T., Pender, S. L., Karjalainen-Lindsberg, M. L., Puolakkainen, P., Macdonald, T. T. \& Saarialho-Kere, U. (2004) Collagenase-1 (MMP-1), matrilysin-1 (MMP-7), and stromelysin-2 (MMP10) are expressed by migrating enterocytes during intestinal wound healing. Scand J Gastroenterol, 39(11), 1095-104.

Sandborn, W. J., Feagan, B. G., Marano, C., Zhang, H., Strauss, R., Johanns, J., Adedokun, O. J., Guzzo, C., Colombel, J. F., Reinisch, W., Gibson, P. R., Collins, J., Jarnerot, G. \& Rutgeerts, P. (2014) Subcutaneous golimumab maintains clinical response in patients with moderate-to-severe ulcerative colitis. Gastroenterology, 146(1), 96-109.e1.

Sandborn, W. J., Feagan, B. G., Rutgeerts, P., Hanauer, S., Colombel, J. F., Sands, B. E., Lukas, M., Fedorak, R. N., Lee, S., Bressler, B., Fox, I., Rosario, M., Sankoh, S., Xu, J., Stephens, K., Milch, C. \& Parikh, A. (2013) Vedolizumab as induction and maintenance therapy for Crohn's disease. $N$ Engl J Med, 369(8), 711-21.

Sandborn, W. J., Hanauer, S. B., Rutgeerts, P., Fedorak, R. N., Lukas, M., MacIntosh, D. G., Panaccione, R., Wolf, D., Kent, J. D., Bittle, B., Li, J. \& Pollack, P. F. (2007) Adalimumab for maintenance treatment of Crohn's disease: results of the CLASSIC II trial. Gut, 56(9), 1232-9.

Sandborn, W. J., van Assche, G., Reinisch, W., Colombel, J. F., D'Haens, G., Wolf, D. C., Kron, M., Tighe, M. B., Lazar, A. \& Thakkar, R. B. (2012) Adalimumab induces and maintains clinical remission in patients with moderate-to-severe ulcerative colitis. Gastroenterology, 142(2), 257-65.e1-3.

Sands, B. E., Anderson, F. H., Bernstein, C. N., Chey, W. Y., Feagan, B. G., Fedorak, R. N., Kamm, M. A., Korzenik, J. R., Lashner, B. A., Onken, J. E., Rachmilewitz, D., Rutgeerts, P., Wild, G., Wolf, D. C., Marsters, P. A., Travers, S. B., Blank, M. A. \& van Deventer, S. J. (2004) Infliximab maintenance therapy for fistulizing Crohn's disease. N Engl J Med, 350(9), 876-85. 
Santana, A., Medina, C., Paz-Cabrera, M. C., Diaz-Gonzalez, F., Farre, E., Salas, A., Radomski, M. W. \& Quintero, E. (2006) Attenuation of dextran sodium sulphate induced colitis in matrix metalloproteinase-9 deficient mice. World J Gastroenterol, 12(40), 6464-72.

Sartor, R. B. (2008) Microbial influences in inflammatory bowel diseases. Gastroenterology, 134(2), 577-94.

Satsangi, J., Chapman, R. W., Haldar, N., Donaldson, P., Mitchell, S., Simmons, J., Norris, S., Marshall, S. E., Bell, J. I., Jewell, D. P. \& Welsh, K. I. (2001) A functional polymorphism of the stromelysin gene (MMP-3) influences susceptibility to primary sclerosing cholangitis. Gastroenterology, 121(1), 124-30. Satsangi, J., Parkes, M., Louis, E., Hashimoto, L., Kato, N., Welsh, K., Terwilliger, J. D., Lathrop, G. M., Bell, J. I. \& Jewell, D. P. (1996) Two stage genome-wide search in inflammatory bowel disease provides evidence for susceptibility loci on chromosomes 3, 7 and 12. Nat Genet, 14(2), 199-202.

Scaldaferri, F., Vetrano, S., Sans, M., Arena, V., Straface, G., Stigliano, E., Repici, A., Sturm, A., Malesci, A., Panes, J., Yla-Herttuala, S., Fiocchi, C. \& Danese, S. (2009) VEGF-A links angiogenesis and inflammation in inflammatory bowel disease pathogenesis. Gastroenterology, 136(2), 585-95.e5.

Scheiffele, F. \& Fuss, I. J. (2002) Induction of TNBS colitis in mice, Curr Protoc Immunol, 2008/04/25 edition, Unit 15.19 .

Schmidt, C., Hauser, W., Giese, T. \& Stallmach, A. (2007) Irritable pouch syndrome is associated with depressiveness and can be differentiated from pouchitis by quantification of mucosal levels of proinflammatory gene transcripts. Inflamm Bowel Dis, 13(12), 1502-8.

Sela-Passwell, N., Kikkeri, R., Dym, O., Rozenberg, H., Margalit, R., Arad-Yellin, R., Eisenstein, M., Brenner, O., Shoham, T., Danon, T., Shanzer, A. \& Sagi, I. (2012) Antibodies targeting the catalytic zinc complex of activated matrix metalloproteinases show therapeutic potential. Nat Med, 18(1), 143-7.

Seo, D. W., Li, H., Guedez, L., Wingfield, P. T., Diaz, T., Salloum, R., Wei, B. Y. \& Stetler-Stevenson, W. G. (2003) TIMP-2 mediated inhibition of angiogenesis: an MMP-independent mechanism. Cell, 114(2), 171-80. 
Sharma, M., Mohapatra, J., Wagh, A., Patel, H. M., Pandey, D., Kadam, S., Argade, A., Deshpande, S. S., Shah, G. B., Chatterjee, A. \& Jain, M. R. (2014) Involvement of TACE in colon inflammation: a novel mechanism of regulation via SIRT-1 activation. Cytokine, 66(1), 30-9.

Shen, B. (2012) Acute and chronic pouchitis--pathogenesis, diagnosis and treatment. Nat Rev Gastroenterol Hepatol, 9(6), 323-33.

Shen, L., Black, E. D., Witkowski, E. D., Lencer, W. I., Guerriero, V., Schneeberger, E. E. \& Turner, J. R. (2006) Myosin light chain phosphorylation regulates barrier function by remodeling tight junction structure. J Cell Sci, 119(Pt 10), 2095-106.

Shi, J., Aono, S., Lu, W., Ouellette, A. J., Hu, X., Ji, Y., Wang, L., Lenz, S., van Ginkel, F. W., Liles, M., Dykstra, C., Morrison, E. E. \& Elson, C. O. (2007) A novel role for defensins in intestinal homeostasis: regulation of IL-1beta secretion. J Immunol, 179(2), 1245-53.

Shirafuji, Y., Tanabe, H., Satchell, D. P., Henschen-Edman, A., Wilson, C. L. \& Ouellette, A. J. (2003) Structural determinants of procryptdin recognition and cleavage by matrix metalloproteinase-7. J Biol Chem, 278(10), 7910-9.

Silosi, I., Boldeanu, M. V., Mogoanta, S. S., Ghilusi, M., Cojocaru, M., Biciusca, V., Cojocaru, I. M., Avramescu, C. S., Gheonea, D. I., Silosi, C. A. \& Turculeanu, A. (2014) Matrix metalloproteinases (MMP-3 and MMP-9) implication in the pathogenesis of inflammatory bowel disease (IBD). Rom J Morphol Embryol, 55(4), 1317-24.

Sim, W. H., Wagner, J., Cameron, D. J., Catto-Smith, A. G., Bishop, R. F. \& Kirkwood, C. D. (2012) Expression profile of genes involved in pathogenesis of pediatric Crohn's disease. J Gastroenterol Hepatol, 27(6), 1083-93.

Singh, S., Al-Darmaki, A., Frolkis, A. D., Seow, C. H., Leung, Y., Novak, K. L., Ghosh, S., Eksteen, B., Panaccione, R. \& Kaplan, G. G. (2015) Postoperative Mortality Among Patients With Inflammatory Bowel Diseases: A Systematic Review and Meta-analysis of Population-Based Studies. Gastroenterology, 149(4), 928-37. 
Sipos, F., Galamb, O., Wichmann, B., Krenacs, T., Toth, K., Leiszter, K., Muzes, G., Zagoni, T., Tulassay, Z. \& Molnar, B. (2011) Peripheral blood based discrimination of ulcerative colitis and Crohn's disease from non-IBD colitis by genome-wide gene expression profiling. Dis Markers, 30(1), 1-17.

Souza, H. S., Tortori, C. J., Castelo-Branco, M. T., Carvalho, A. T., Margallo, V. S., Delgado, C. F., Dines, I. \& Elia, C. C. (2005) Apoptosis in the intestinal mucosa of patients with inflammatory bowel disease: evidence of altered expression of FasL and perforin cytotoxic pathways. Int J Colorectal Dis, 20(3), 277-86.

Stallmach, A., Chan, C. C., Ecker, K. W., Feifel, G., Herbst, H., Schuppan, D. \& Zeitz, M. (2000) Comparable expression of matrix metalloproteinases 1 and 2 in pouchitis and ulcerative colitis. Gut, 47(3), 415-22.

Sternlicht, M. D. \& Werb, Z. (2001) How matrix metalloproteinases regulate cell behavior. Annu Rev Cell Dev Biol, 17, 463-516.

Stetler-Stevenson, W. G. (2008) Tissue inhibitors of metalloproteinases in cell signaling: metalloproteinase-independent biological activities. Sci Signal, 1(27), re6.

Strober, W. (1985) Animal models of inflammatory bowel disease--an overview. Dig Dis Sci, 30(12 Suppl), 3s-10s.

Strongin, A. Y., Collier, I., Bannikov, G., Marmer, B. L., Grant, G. A. \& Goldberg, G. I. (1995) Mechanism of cell surface activation of 72-kDa type IV collagenase. Isolation of the activated form of the membrane metalloprotease. J Biol Chem, 270(10), 5331-8.

Stumpf, M., Cao, W., Klinge, U., Klosterhalfen, B., Junge, K., Krones, C. J. \& Schumpelick, V. (2005) Reduced expression of collagen type I and increased expression of matrix metalloproteinases 1 in patients with Crohn's disease. J Invest Surg, 18(1), 33-8.

Sun, M., He, C., Cong, Y. \& Liu, Z. (2015) Regulatory immune cells in regulation of intestinal inflammatory response to microbiota. Mucosal Immunol, 8(5), 969-78. 
Swee, M., Wilson, C. L., Wang, Y., McGuire, J. K. \& Parks, W. C. (2008) Matrix metalloproteinase-7 (matrilysin) controls neutrophil egress by generating chemokine gradients. J Leukoc Biol, 83(6), 140412.

Sykes, A. P., Bhogal, R., Brampton, C., Chander, C., Whelan, C., Parsons, M. E. \& Bird, J. (1999) The effect of an inhibitor of matrix metalloproteinases on colonic inflammation in a trinitrobenzenesulphonic acid rat model of inflammatory bowel disease. Aliment Pharmacol Ther, 13(11), 1535-42.

Tall, A. R. (2004) C-reactive protein reassessed. N Engl J Med, 350(14), 1450-2.

Tan, G., Zeng, B. \& Zhi, F. C. (2015) Regulation of human enteric alpha-defensins by NOD2 in the Paneth cell lineage. Eur J Cell Biol, 94(1), 60-6.

Tanaka, M., Itai, T., Adachi, M. \& Nagata, S. (1998) Downregulation of Fas ligand by shedding. Nat Med, 4(1), 31-6.

Tarlton, J. F., Whiting, C. V., Tunmore, D., Bregenholt, S., Reimann, J., Claesson, M. H. \& Bland, P. W. (2000) The role of up-regulated serine proteases and matrix metalloproteinases in the pathogenesis of a murine model of colitis. Am J Pathol, 157(6), 1927-35.

ten Hove, T., van Montfrans, C., Peppelenbosch, M. P. \& van Deventer, S. J. (2002) Infliximab treatment induces apoptosis of lamina propria T lymphocytes in Crohn's disease. Gut, 50(2), 206-11.

Thathiah, A. \& Carson, D. D. (2004) MT1-MMP mediates MUC1 shedding independent of TACE/ADAM17. Biochem J, 382(Pt 1), 363-73.

Thomas, C. W., Myhre, G. M., Tschumper, R., Sreekumar, R., Jelinek, D., McKean, D. J., Lipsky, J. J., Sandborn, W. J. \& Egan, L. J. (2005) Selective inhibition of inflammatory gene expression in activated T lymphocytes: a mechanism of immune suppression by thiopurines. J Pharmacol Exp Ther, 312(2), $537-45$.

Tolstanova, G., Deng, X., Khomenko, T., Garg, P., Paunovic, B., Chen, L., Sitaraman, S. V., Shiloach, J., Szabo, S. \& Sandor, Z. (2011) Role of anti-angiogenic factor endostatin in the pathogenesis of experimental ulcerative colitis. Life Sci, 88(1-2), 74-81. 
Tolstanova, G., Khomenko, T., Deng, X., Chen, L., Tarnawski, A., Ahluwalia, A., Szabo, S. \& Sandor, Z. (2009) Neutralizing anti-vascular endothelial growth factor (VEGF) antibody reduces severity of experimental ulcerative colitis in rats: direct evidence for the pathogenic role of VEGF. $J$ Pharmacol Exp Ther, 328(3), 749-57.

Tomita, T., Fujii, M., Tokumaru, Y., Imanishi, Y., Kanke, M., Yamashita, T., Ishiguro, R., Kanzaki, J., Kameyama, K. \& Otani, Y. (2000) Granulocyte-macrophage colony-stimulating factor upregulates matrix metalloproteinase-2 (MMP-2) and membrane type-1 MMP (MT1-MMP) in human head and neck cancer cells. Cancer Lett, 156(1), 83-91.

Trivedi, P. P. \& Jena, G. B. (2013) Role of alpha-lipoic acid in dextran sulfate sodium-induced ulcerative colitis in mice: studies on inflammation, oxidative stress, DNA damage and fibrosis. Food Chem Toxicol, 59, 339-55.

Tsagaraki, I., Tsilibary, E. C. \& Tzinia, A. K. (2010) TIMP-1 interaction with alphavbeta3 integrin confers resistance to human osteosarcoma cell line MG-63 against TNF-alpha-induced apoptosis. Cell Tissue Res, 342(1), 87-96.

Turner, J. R. (2006) Molecular basis of epithelial barrier regulation: from basic mechanisms to clinical application. Am J Pathol, 169(6), 1901-9.

Tysk, C., Lindberg, E., Jarnerot, G. \& Floderus-Myrhed, B. (1988) Ulcerative colitis and Crohn's disease in an unselected population of monozygotic and dizygotic twins. A study of heritability and the influence of smoking. Gut, 29(7), 990-6.

Udi, Y., Fragai, M., Grossman, M., Mitternacht, S., Arad-Yellin, R., Calderone, V., Melikian, M., Toccafondi, M., Berezovsky, I. N., Luchinat, C. \& Sagi, I. (2013) Unraveling hidden regulatory sites in structurally homologous metalloproteases. J Mol Biol, 425(13), 2330-46.

Vaalamo, M., Karjalainen-Lindsberg, M. L., Puolakkainen, P., Kere, J. \& Saarialho-Kere, U. (1998) Distinct expression profiles of stromelysin-2 (MMP-10), collagenase-3 (MMP-13), macrophage metalloelastase (MMP-12), and tissue inhibitor of metalloproteinases-3 (TIMP-3) in intestinal ulcerations. Am J Pathol, 152(4), 1005-14. 
Valatas, V., Vakas, M. \& Kolios, G. (2013) The value of experimental models of colitis in predicting efficacy of biological therapies for inflammatory bowel diseases. Am J Physiol Gastrointest Liver Physiol, 305(11), G763-85.

Van Assche, G. \& Rutgeerts, P. (2004) Medical management of postoperative recurrence in Crohn's disease. Gastroenterol Clin North Am, 33(2), 347-60, x.

Van Assche, G., Vermeire, S. \& Rutgeerts, P. (2009) Infliximab therapy for patients with inflammatory bowel disease: 10 years on. Eur J Pharmacol, 623 Suppl 1, S17-25.

Van Damme, J., Van Beeumen, J., Opdenakker, G. \& Billiau, A. (1988) A novel, NH2-terminal sequence-characterized human monokine possessing neutrophil chemotactic, skin-reactive, and granulocytosis-promoting activity. J Exp Med, 167(4), 1364-76.

van den Brande, J., Hommes, D. W. \& Peppelenbosch, M. P. (2005) Infliximab induced T lymphocyte apoptosis in Crohn's disease. J Rheumatol Suppl, 74, 26-30.

Van den Brande, J. M., Braat, H., van den Brink, G. R., Versteeg, H. H., Bauer, C. A., Hoedemaeker, I., van Montfrans, C., Hommes, D. W., Peppelenbosch, M. P. \& van Deventer, S. J. (2003) Infliximab but not etanercept induces apoptosis in lamina propria T-lymphocytes from patients with Crohn's disease. Gastroenterology, 124(7), 1774-85.

Van den Steen, P. E., Dubois, B., Nelissen, I., Rudd, P. M., Dwek, R. A. \& Opdenakker, G. (2002) Biochemistry and molecular biology of gelatinase B or matrix metalloproteinase-9 (MMP-9). Crit Rev Biochem Mol Biol, 37(6), 375-536.

Van den Steen, P. E., Proost, P., Wuyts, A., Van Damme, J. \& Opdenakker, G. (2000) Neutrophil gelatinase B potentiates interleukin-8 tenfold by aminoterminal processing, whereas it degrades CTAPIII, PF-4, and GRO-alpha and leaves RANTES and MCP-2 intact. Blood, 96(8), 2673-81.

Van der Sluis, M., De Koning, B. A., De Bruijn, A. C., Velcich, A., Meijerink, J. P., Van Goudoever, J. B., Buller, H. A., Dekker, J., Van Seuningen, I., Renes, I. B. \& Einerhand, A. W. (2006) Muc2-deficient mice spontaneously develop colitis, indicating that MUC2 is critical for colonic protection. Gastroenterology, 131(1), 117-29. 
Van Limbergen, J., Radford-Smith, G. \& Satsangi, J. (2014) Advances in IBD genetics. Nat Rev Gastroenterol Hepatol, 11(6), 372-85.

Van Ranst, M., Norga, K., Masure, S., Proost, P., Vandekerckhove, F., Auwerx, J., Van Damme, J. \& Opdenakker, G. (1991) The cytokine-protease connection: identification of a 96-kD THP-1 gelatinase and regulation by interleukin-1 and cytokine inducers. Cytokine, 3(3), 231-9.

Van Wart, H. E. \& Birkedal-Hansen, H. (1990) The cysteine switch: a principle of regulation of metalloproteinase activity with potential applicability to the entire matrix metalloproteinase gene family. Proc Natl Acad Sci U S A, 87(14), 5578-82.

Vande Casteele, N., Ferrante, M., Van Assche, G., Ballet, V., Compernolle, G., Van Steen, K., Simoens, S., Rutgeerts, P., Gils, A. \& Vermeire, S. (2015) Trough concentrations of infliximab guide dosing for patients with inflammatory bowel disease. Gastroenterology, 148(7), 1320-9.e3.

Vanden Berghe, T., Hulpiau, P., Martens, L., Vandenbroucke, R. E., Van Wonterghem, E., Perry, S. W., Bruggeman, I., Divert, T., Choi, S. M., Vuylsteke, M., Shestopalov, V. I., Libert, C. \& Vandenabeele, P. (2015) Passenger Mutations Confound Interpretation of All Genetically Modified Congenic Mice. Immunity, 43(1), 200-9.

Vandenbroucke, R. E., Dejonckheere, E., Van Hauwermeiren, F., Lodens, S., De Rycke, R., Van Wonterghem, E., Staes, A., Gevaert, K., Lopez-Otin, C. \& Libert, C. (2013) Matrix metalloproteinase 13 modulates intestinal epithelial barrier integrity in inflammatory diseases by activating TNF. EMBO Mol Med, 5(7), 932-48.

Vandenbroucke, R. E. \& Libert, C. (2014) Is there new hope for therapeutic matrix metalloproteinase inhibition? Nat Rev Drug Discov, 13(12), 904-27.

Vandenbroucke, R. E., Vanlaere, I., Van Hauwermeiren, F., Van Wonterghem, E., Wilson, C. \& Libert, C. (2014) Pro-inflammatory effects of matrix metalloproteinase 7 in acute inflammation. Mucosal Immunol, 7(3), 579-88. 
Vandooren, J., Geurts, N., Martens, E., Van den Steen, P. E., Jonghe, S. D., Herdewijn, P. \& Opdenakker, G. (2011) Gelatin degradation assay reveals MMP-9 inhibitors and function of Oglycosylated domain. World J Biol Chem, 2(1), 14-24.

Vandooren, J., Geurts, N., Martens, E., Van den Steen, P. E. \& Opdenakker, G. (2013a) Zymography methods for visualizing hydrolytic enzymes. Nat Methods, 10(3), 211-20.

Vandooren, J., Opdenakker, G., Loadman, P. M. \& Edwards, D. R. (2016) Proteases in cancer drug delivery. Adv Drug Deliv Rev, 97, 144-55.

Vandooren, J., Van den Steen, P. E. \& Opdenakker, G. (2013b) Biochemistry and molecular biology of gelatinase B or matrix metalloproteinase-9 (MMP-9): the next decade. Crit Rev Biochem Mol Biol, 48(3), 222-72.

Visse, R. \& Nagase, H. (2003) Matrix metalloproteinases and tissue inhibitors of metalloproteinases: structure, function, and biochemistry. Circ Res, 92(8), 827-39.

Vizoso, F. J., Gonzalez, L. O., Corte, M. D., Corte, M. G., Bongera, M., Martinez, A., Martin, A., Andicoechea, A. \& Gava, R. R. (2006) Collagenase-3 (MMP-13) expression by inflamed mucosa in inflammatory bowel disease. Scand J Gastroenterol, 41(9), 1050-5.

von Lampe, B., Barthel, B., Coupland, S. E., Riecken, E. O. \& Rosewicz, S. (2000) Differential expression of matrix metalloproteinases and their tissue inhibitors in colon mucosa of patients with inflammatory bowel disease. Gut, 47(1), 63-73.

von Stein, P., Lofberg, R., Kuznetsov, N. V., Gielen, A. W., Persson, J. O., Sundberg, R., Hellstrom, K., Eriksson, A., Befrits, R., Ost, A. \& von Stein, O. D. (2008) Multigene analysis can discriminate between ulcerative colitis, Crohn's disease, and irritable bowel syndrome. Gastroenterology, 134(7), 1869-81; quiz 2153-4.

Wang, Y. D. \& Mao, J. W. (2007) Expression of matrix metalloproteinase-1 and tumor necrosis factoralpha in ulcerative colitis. World J Gastroenterol, 13(44), 5926-32. 
Wang, Y. D. \& Wang, W. (2008) Protective effect of ilomastat on trinitrobenzenesulfonic acid-induced ulcerative colitis in rats. World J Gastroenterol, 14(37), 5683-8.

Wang, Y. D. \& Yan, P. Y. (2006) Expression of matrix metalloproteinase-1 and tissue inhibitor of metalloproteinase-1 in ulcerative colitis. World J Gastroenterol, 12(37), 6050-3.

Ward, R. V., Atkinson, S. J., Reynolds, J. J. \& Murphy, G. (1994) Cell surface-mediated activation of progelatinase A: demonstration of the involvement of the C-terminal domain of progelatinase A in cell surface binding and activation of progelatinase A by primary fibroblasts. Biochem J, 304 ( Pt 1), 263-9. Weathington, N. M., van Houwelingen, A. H., Noerager, B. D., Jackson, P. L., Kraneveld, A. D., Galin, F. S., Folkerts, G., Nijkamp, F. P. \& Blalock, J. E. (2006) A novel peptide CXCR ligand derived from extracellular matrix degradation during airway inflammation. Nat Med, 12(3), 317-23.

Weber, C. R., Nalle, S. C., Tretiakova, M., Rubin, D. T. \& Turner, J. R. (2008) Claudin-1 and claudin2 expression is elevated in inflammatory bowel disease and may contribute to early neoplastic transformation. Lab Invest, 88(10), 1110-20.

Werner, T., Wagner, S. J., Martinez, I., Walter, J., Chang, J. S., Clavel, T., Kisling, S., Schuemann, K. \& Haller, D. (2011) Depletion of luminal iron alters the gut microbiota and prevents Crohn's diseaselike ileitis. Gut, 60(3), 325-33.

Wiercinska-Drapalo, A., Jaroszewicz, J., Flisiak, R. \& Prokopowicz, D. (2003) Plasma matrix metalloproteinase-1 and tissue inhibitor of metalloproteinase-1 as biomarkers of ulcerative colitis activity. World J Gastroenterol, 9(12), 2843-5.

Wilson, C. L., Ouellette, A. J., Satchell, D. P., Ayabe, T., Lopez-Boado, Y. S., Stratman, J. L., Hultgren, S. J., Matrisian, L. M. \& Parks, W. C. (1999) Regulation of intestinal alpha-defensin activation by the metalloproteinase matrilysin in innate host defense. Science, 286(5437), 113-7.

Wirtz, S. \& Neurath, M. F. (2000) Animal models of intestinal inflammation: new insights into the molecular pathogenesis and immunotherapy of inflammatory bowel disease. Int J Colorectal Dis, 15(3), $144-60$. 
Wirtz, S. \& Neurath, M. F. (2007) Mouse models of inflammatory bowel disease. Adv Drug Deliv Rev, 59(11), 1073-83.

Woessner, J. F., Jr. (2002) MMPs and TIMPs--an historical perspective. Mol Biotechnol, 22(1), 33-49.

Wu, X. F., Fei, M. J., Shu, R. G., Tan, R. X. \& Xu, Q. (2005) Fumigaclavine C, an fungal metabolite, improves experimental colitis in mice via downregulating Th1 cytokine production and matrix metalloproteinase activity. Int Immunopharmacol, 5(10), 1543-53.

Yadav, M., Huang, M. C. \& Goetzl, E. J. (2011) VPAC1 (vasoactive intestinal peptide (VIP) receptor type 1) G protein-coupled receptor mediation of VIP enhancement of murine experimental colitis. Cell Immunol, 267(2), 124-32.

Yamada, A., Uegaki, A., Nakamura, T. \& Ogawa, K. (2000) ONO-4817, an orally active matrix metalloproteinase inhibitor, prevents lipopolysaccharide-induced proteoglycan release from the joint cartilage in guinea pigs. Inflamm Res, 49(4), 144-6.

Yamaguchi, H., Suzuki, K., Nagata, M., Kawase, T., Sukumaran, V., Thandavarayan, R. A., Kawauchi, Y., Yokoyama, J., Tomita, M., Kawachi, H., Watanabe, K., Yoneyama, H., Asakura, H. \& Takagi, R. (2012) Irsogladine maleate ameliorates inflammation and fibrosis in mice with chronic colitis induced by dextran sulfate sodium. Med Mol Morphol, 45(3), 140-51.

Yan, C. \& Boyd, D. D. (2007) Regulation of matrix metalloproteinase gene expression. J Cell Physiol, 211(1), 19-26.

Yanai, H. \& Hanauer, S. B. (2011) Assessing response and loss of response to biological therapies in IBD. Am J Gastroenterol, 106(4), 685-98.

Yukawa, M., Iizuka, M., Horie, Y., Yoneyama, K., Shirasaka, T., Itou, H., Komatsu, M., Fukushima, T. $\&$ Watanabe, S. (2002) Systemic and local evidence of increased Fas-mediated apoptosis in ulcerative colitis. Int J Colorectal Dis, 17(2), 70-6. 


\section{TABLES}

Table 1: Overview of the most commonly used IBD mouse models. Table adapted from (Jones-Hall \& Grisham, 2014).

\begin{tabular}{|c|c|c|c|c|c|}
\hline Category & $\begin{array}{l}\text { Model } \\
\text { system }\end{array}$ & $\begin{array}{l}\text { Human } \\
\text { disease }\end{array}$ & $\begin{array}{l}\text { Predominant cytokine } \\
\text { response }\end{array}$ & $\begin{array}{l}\text { Location of } \\
\text { lesions in } \\
\text { intestine }\end{array}$ & $\begin{array}{l}\text { Pathologic response in } \\
\text { intestine }\end{array}$ \\
\hline $\begin{array}{l}\text { Genetically } \\
\text { engineered }\end{array}$ & $\begin{array}{l}\text { IL-10 }{ }^{-/} \\
\text {mouse }\end{array}$ & $\mathrm{CD}$ & $\begin{array}{l}\text { Th1 } \\
\uparrow: \text { TNF, IL-1 } \beta, \text { IFN- } \gamma\end{array}$ & $\begin{array}{l}\text { Entire small } \\
\text { and large } \\
\text { intestine } \\
\text { (duodenum } \\
\text { most severe) }\end{array}$ & $\begin{array}{l}\text { Mononuclear to granulomatous } \\
\text { inflammation, } \\
\text { mucosal/submucosal, epithelial } \\
\text { regeneration, pseudeopolyps }\end{array}$ \\
\hline $\begin{array}{l}\text { Genetically } \\
\text { engineered }\end{array}$ & $\begin{array}{l}\mathrm{TNF}^{\triangle \mathrm{ARE}} \\
\text { mouse }\end{array}$ & $\mathrm{CD}$ & $\begin{array}{l}\text { Th1/Th17 } \\
\uparrow: \text { TNF, IL-17, IFN- } \gamma, \text { IL-10 }\end{array}$ & Ileum & $\begin{array}{l}\text { Blunting and widening of villi, } \\
\text { transmural and mixed } \\
\text { inflammation with non- } \\
\text { caseating granulomas }\end{array}$ \\
\hline $\begin{array}{l}\text { Immune } \\
\text { manipulated }\end{array}$ & $\begin{array}{l}\text { T cell } \\
\text { transfer }\end{array}$ & $\mathrm{CD} / \mathrm{UC}$ & $\begin{array}{l}\text { Th1/Th17 } \\
\uparrow: \text { TNF, IFN- } \gamma \\
\downarrow: \text { IL-10, IL-4 } \\
\uparrow / \downarrow: \text { IL-17 }\end{array}$ & $\begin{array}{l}\text { Distal colon, } \\
\text { small intestine }\end{array}$ & $\begin{array}{l}\text { Mixed (macrophages, } \\
\text { lymphocytes, neutrophils) } \\
\text { inflammation, } \\
\text { mucosal/submucosal, goblet cell } \\
\text { loss, ulceration }\end{array}$ \\
\hline Spontaneous & $\begin{array}{l}\mathrm{SAMP} 1 / \mathrm{Yi} \\
\mathrm{t}(\mathrm{Fc}) \\
\text { mouse }\end{array}$ & $\mathrm{CD} / \mathrm{UC}$ & $\begin{array}{l}\text { Th1/Th2 } \\
\uparrow: \text { TNF, IFN- } \gamma \\
\uparrow: \text { IL-33, IL-17, IL-4, IL-10 }\end{array}$ & $\begin{array}{l}\text { Terminal ileum } \\
\text { (cecum) }\end{array}$ & $\begin{array}{l}\text { Mixed (macrophages, } \\
\text { lymphocytes, neutrophils) } \\
\text { inflammation, crypt } \\
\text { microabsseses, granulomas }\end{array}$ \\
\hline $\begin{array}{l}\text { Chemically } \\
\text { induced }\end{array}$ & $\begin{array}{l}\text { DSS } \\
\text { model }\end{array}$ & $\mathrm{UC}$ & $\begin{array}{l}\text { Acute: Th1/Th17 (Th2) } \\
\uparrow: \text { TNF, IFN- } \gamma, \text { IL-12, IL-1, } \\
\text { IL-4, IL-17, KC } \\
\text { Chronic: Th2 (Th1) } \\
\uparrow: \text { IFN- } \gamma, \text { IL-6, IL-10, IL-4 } \\
\downarrow: \text { TNF, IL-6, IL17 }\end{array}$ & $\begin{array}{l}\text { Colon (location } \\
\text { varies) }\end{array}$ & $\begin{array}{l}\text { Acute: Mixed (neutrophils } \\
\text { predominate), superficial, } \\
\text { ulceration and necrosis } \\
\text { Chronic: Patchy inflammation, } \\
\text { prominent lymphoid follicles, } \\
\text { fissuring ulcers }\end{array}$ \\
\hline $\begin{array}{l}\text { Chemically } \\
\text { induced }\end{array}$ & $\begin{array}{l}\text { TNBS } \\
\text { model }\end{array}$ & $\mathrm{CD}$ & $\begin{array}{l}\text { Th1/Th17 } \\
\uparrow: \text { TNF, IL-18, IL-2, IFN- } \gamma, \\
\text { IL-12, IL-6 } \\
\downarrow: \text { IL-4 }\end{array}$ & Entire colon & $\begin{array}{l}\text { Mononuclear to granulomatous } \\
\text { inflammation, transmural, } \\
\text { fibrosis }\end{array}$ \\
\hline
\end{tabular}

Primary cytokine responses are shown in bold, whereas secondary effects are shown in normal font. Arrows indicate increased $(\uparrow)$ or decreased $(\downarrow)$ expression of cytokines. 
Table 2: MMP polymorphism associations with IBD.

\begin{tabular}{|c|c|c|c|c|c|}
\hline Gene & Alternative name & SNP & $\begin{array}{l}\text { Human } \\
\text { genotype }\end{array}$ & Main findings & References \\
\hline \multirow[t]{3}{*}{ MMP-1 } & \multirow[t]{3}{*}{$\begin{array}{l}\text { Interstitial } \\
\text { collagenase }\end{array}$} & \multirow[t]{3}{*}{ rs1799750 } & $-16071 \mathrm{G} / 2 \mathrm{G}$ & Not associated with IBD & $\begin{array}{l}\text { (Meijer et al, } \\
\text { 2007c; Morgan } \\
\text { et al, 2011; } \\
\text { Satsangi et al, } \\
\text { 2001) }\end{array}$ \\
\hline & & & $\begin{array}{l}-16071 \mathrm{G} 2 \mathrm{G} \text { or } \\
2 \mathrm{G} 2 \mathrm{G}\end{array}$ & $\begin{array}{l}\text { Higher MMP-1 expression in IBD patients with at least one } \\
2 \mathrm{G} \text { allele } \\
\text { Lowered MMP-1 expression in IBD patients with } 1 \mathrm{G} 2 \mathrm{G} \text { or } \\
2 \mathrm{G} 2 \mathrm{G} \text { alleles after treatment with infliximab }\end{array}$ & $\begin{array}{l}\text { (Meijer et al, } \\
2007 \mathrm{c})\end{array}$ \\
\hline & & & $-1607-/ \mathrm{G}$ & Not associated with collagenous colitis & $\begin{array}{l}\text { (Madisch et al, } \\
\text { 2011) }\end{array}$ \\
\hline MMP-2 & Gelatinase A & rs243865 & $-1306 \mathrm{C} / \mathrm{T}$ & Not associated with IBD & $\begin{array}{l}\text { (Meijer et al, } \\
\text { 2007c; Morgan }\end{array}$ \\
\hline
\end{tabular}




\begin{tabular}{|c|c|c|c|c|c|}
\hline & & & & & $\begin{array}{l}\text { et al, 2011; } \\
\text { Satsangi et al, } \\
\text { 2001) }\end{array}$ \\
\hline & & & $-13062 \mathrm{C}$ & Higher MMP-2 expression in IBD patients with $2 \mathrm{C}$ alleles & $\begin{array}{l}\text { (Meijer et al, } \\
2007 \mathrm{c})\end{array}$ \\
\hline \multirow[t]{6}{*}{ MMP-3 } & \multirow[t]{6}{*}{ Stromelysin 1} & \multirow[t]{6}{*}{ rs 3025058} & $-11715 \mathrm{~A} / 6 \mathrm{~A}$ & Associated with PSC, not associated with UC alone & $\begin{array}{l}\text { (Satsangi et al, } \\
\text { 2001) }\end{array}$ \\
\hline & & & $-11715 \mathrm{~A} / 5 \mathrm{~A}$ & Associated with CD & $\begin{array}{l}\text { (Pender et al, } \\
\text { 2004) }\end{array}$ \\
\hline & & & $-16135 \mathrm{~T} / 6 \mathrm{~T}$ & Not associated with IBD & \multirow{3}{*}{$\begin{array}{l}\text { (Meijer et al, } \\
2007 \mathrm{c})\end{array}$} \\
\hline & & & $-16135 \mathrm{~T} / 5 \mathrm{~T}$ & Associated with CD & \\
\hline & & & $-16135 \mathrm{~T}$ & $\begin{array}{l}\text { Higher MMP-3 expression in IBD patients with at least one } \\
5 \text { T allele }\end{array}$ & \\
\hline & & & $-1613 \mathrm{~T}$ & Associated with UC & $\begin{array}{l}\text { (Morgan et al, } \\
\text { 2011) }\end{array}$ \\
\hline
\end{tabular}




\begin{tabular}{|c|c|c|c|c|c|}
\hline & & rs678815 & $\mathrm{G} / \mathrm{G}$ & $\begin{array}{l}\text { Associated with UC / females, age at first diagnosis between } \\
17 \text { and } 40 \text { years, left colon disease, smoking at diagnosis, } \\
\text { family and relatives with IBD }\end{array}$ & $\begin{array}{l}\text { (Morgan et al, } \\
\text { 2011) }\end{array}$ \\
\hline \multirow[t]{2}{*}{ MMP-7 } & Matrilysin 1 & NA & $-153 \mathrm{C} / \mathrm{T}$ & Not associated with collagenous colitis & \multirow{2}{*}{$\begin{array}{l}\text { (Madisch et al, } \\
\text { 2011) }\end{array}$} \\
\hline & & NA & $-181 \mathrm{~A} / \mathrm{G}$ & Not associated with collagenous colitis & \\
\hline \multirow[t]{3}{*}{ MMP-8 } & $\begin{array}{l}\text { Neutrophil } \\
\text { collagenase }\end{array}$ & rs1276284 & $\mathrm{C} / \mathrm{C}$ & $\begin{array}{l}\text { Associated with lowered risk for UC / females, patients } \\
\text { diagnosed after } 40 \text { years of age and between } 17 \text { and } 40 \text { years } \\
\text { of age, pancolitis, bowel resection, extra-intestinal } \\
\text { manifestations and smoking at diagnosis }\end{array}$ & \multirow{3}{*}{$\begin{array}{l}\text { (Morgan et al, } \\
\text { 2011) }\end{array}$} \\
\hline & & rs11225394 & $\mathrm{G} / \mathrm{G}$ & $\begin{array}{l}\text { Associated with lowered risk for UC / females and patients } \\
\text { diagnosed after } 40 \text { years of age }\end{array}$ & \\
\hline & & rs3765620 & $\mathrm{A} / \mathrm{A}$ & $\begin{array}{l}\text { Associated with lowered risk for UC / females, patients } \\
\text { diagnosed after } 40 \text { years of age and disease of the left colon }\end{array}$ & \\
\hline MMP-9 & Gelatinase B & rs3918278 & $-1562 \mathrm{C} / \mathrm{T}$ & Not associated with IBD & $\begin{array}{l}\text { (Meijer et al, } \\
\text { 2007c; Morgan } \\
\text { et al, 2011; }\end{array}$ \\
\hline
\end{tabular}




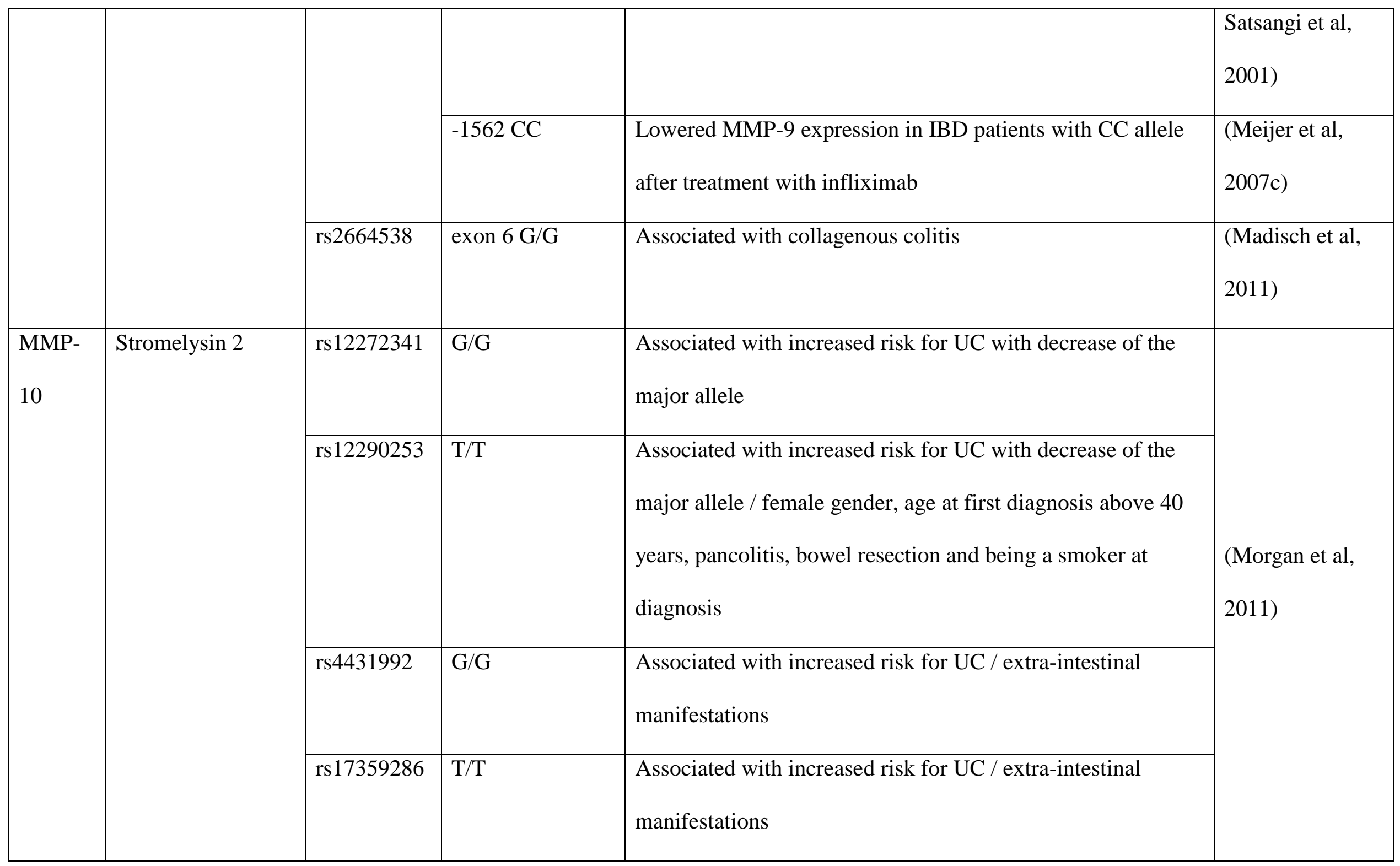




\begin{tabular}{|l|l|l|l|l|l|}
\hline & & rs470155 & A/A & Associated with increased risk for UC / extra-intestinal & manifestations \\
13 & & & & Not associated with IBD & (Morgan et al, \\
MMP- & Collagenase 3 & rs2252070 & -77 A/G & Associated with UC & (Morgan et al, \\
\hline 14 & MT1 MMP & rs3751488 & G/G & & 2011) \\
\hline
\end{tabular}

Abbreviations: NA, not applicable 
Table 3: Overview of MMP mRNA expression studies in human samples.

\begin{tabular}{|c|c|c|c|c|}
\hline MMP & Method & Sample/IBD type & Main findings & References \\
\hline $\begin{array}{l}\text { MMP-1, }-2, \quad-3, \quad-9 \\
\text { and MT1-MMP }\end{array}$ & qRT-PCR, ISH & $\begin{array}{l}\text { Human fetal mesenchymal cell } \\
\text { line }\end{array}$ & $\begin{array}{l}\uparrow M T 1-M M P \text { and } M M P-3 \text { in fibroblast at ulcer } \\
\text { edge }\end{array}$ & $\begin{array}{l}\text { (Pender et al, } \\
2000)\end{array}$ \\
\hline MMP-9 & qRT-PCR & Caco- 2 cell line & $\begin{array}{l}\underline{\mathrm{IL}-1 \beta \text { and TNF- } \alpha \text { stimulation: }} \uparrow M M P-9 \\
\underline{\mathrm{LPS}}:=M M P-9 \\
\underline{\text { PPAR- } \gamma \text { agonist: }} \downarrow \downarrow M M P-9\end{array}$ & (Gan et al, 2001) \\
\hline MMP-1, -2, -3, -9 & qRT-PCR & $\begin{array}{l}\text { Myofibroblast cultures and } \\
\text { mucosal samples (UC and CD) }\end{array}$ & $\begin{array}{l}=M M P-1,-2 \text { and }-3 \\
M M P-9 \text { undetectable }\end{array}$ & $\begin{array}{l}\text { (McKaig et al, } \\
\text { 2003) }\end{array}$ \\
\hline
\end{tabular}




\begin{tabular}{|c|c|c|c|c|}
\hline MMP-1, -2, -3, -9 & qRT-PCR & $\begin{array}{l}\text { Intestinal fibroblast cultures } \\
\text { and mucosal samples (CD) }\end{array}$ & $\begin{array}{l}\text { Control: } M M P-1,-2,-3,-9 \text { detected } \\
\underline{\text { IL-21 stimulation: }=}\end{array}$ & $\begin{array}{l}\text { (Monteleone et al, } \\
\text { 2006) }\end{array}$ \\
\hline MMP-2 and -9 & qRT-PCR & Whole blood (CD) & $\begin{array}{l}\underline{\text { LPS }}: \uparrow M M P-9(16 \text {-fold }) \\
\underline{\text { Infliximab: }} \downarrow \downarrow M M P-9(80 \%)\end{array}$ & (Gao et al, 2007) \\
\hline MMP-3 & qRT-PCR & $\begin{array}{l}\text { Gut PCs isolated from colonic } \\
\text { tissue (UC and CD) }\end{array}$ & $\uparrow M M P-3$ & $\begin{array}{l}\text { (Gordon et al, } \\
2008)\end{array}$ \\
\hline
\end{tabular}




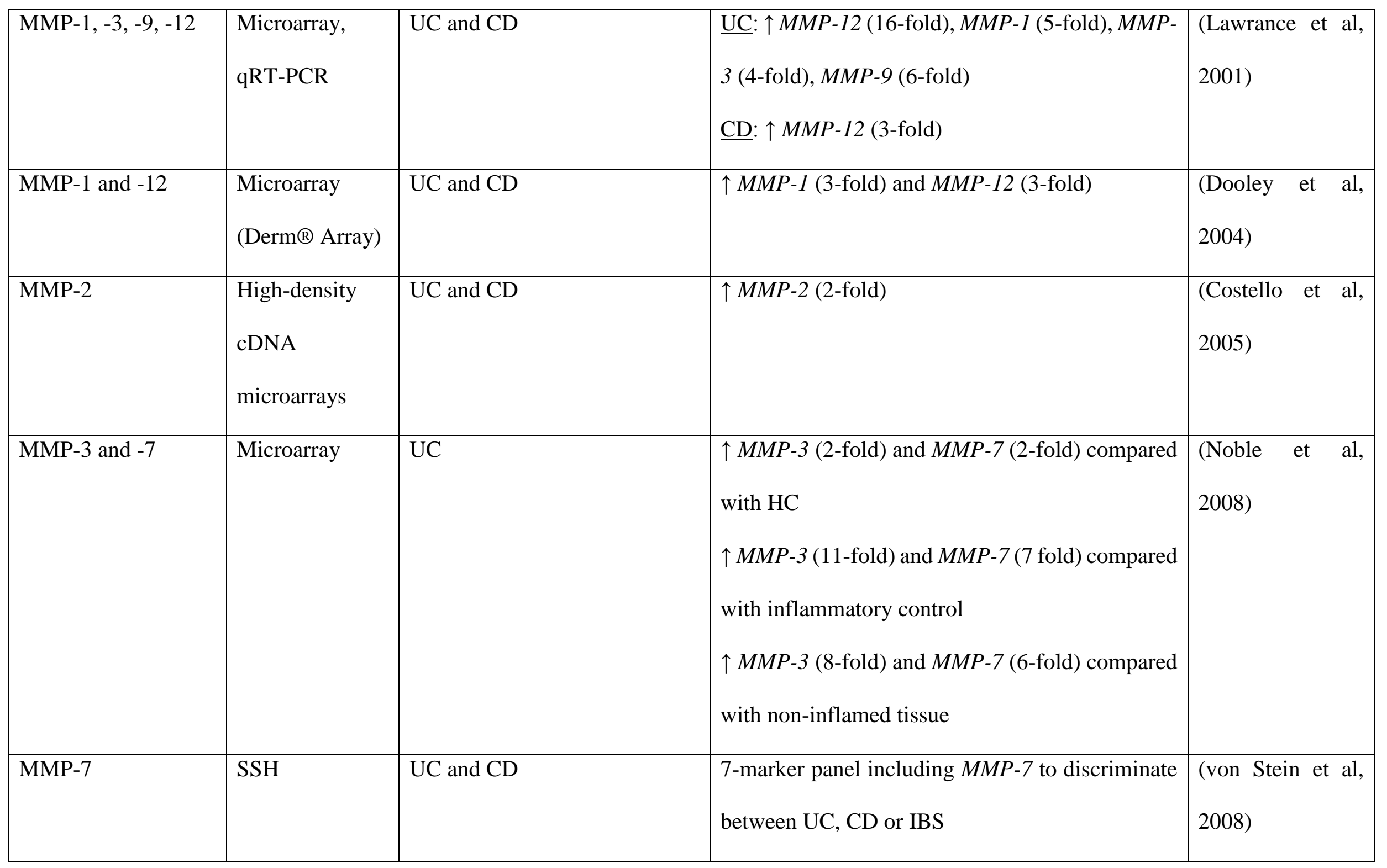




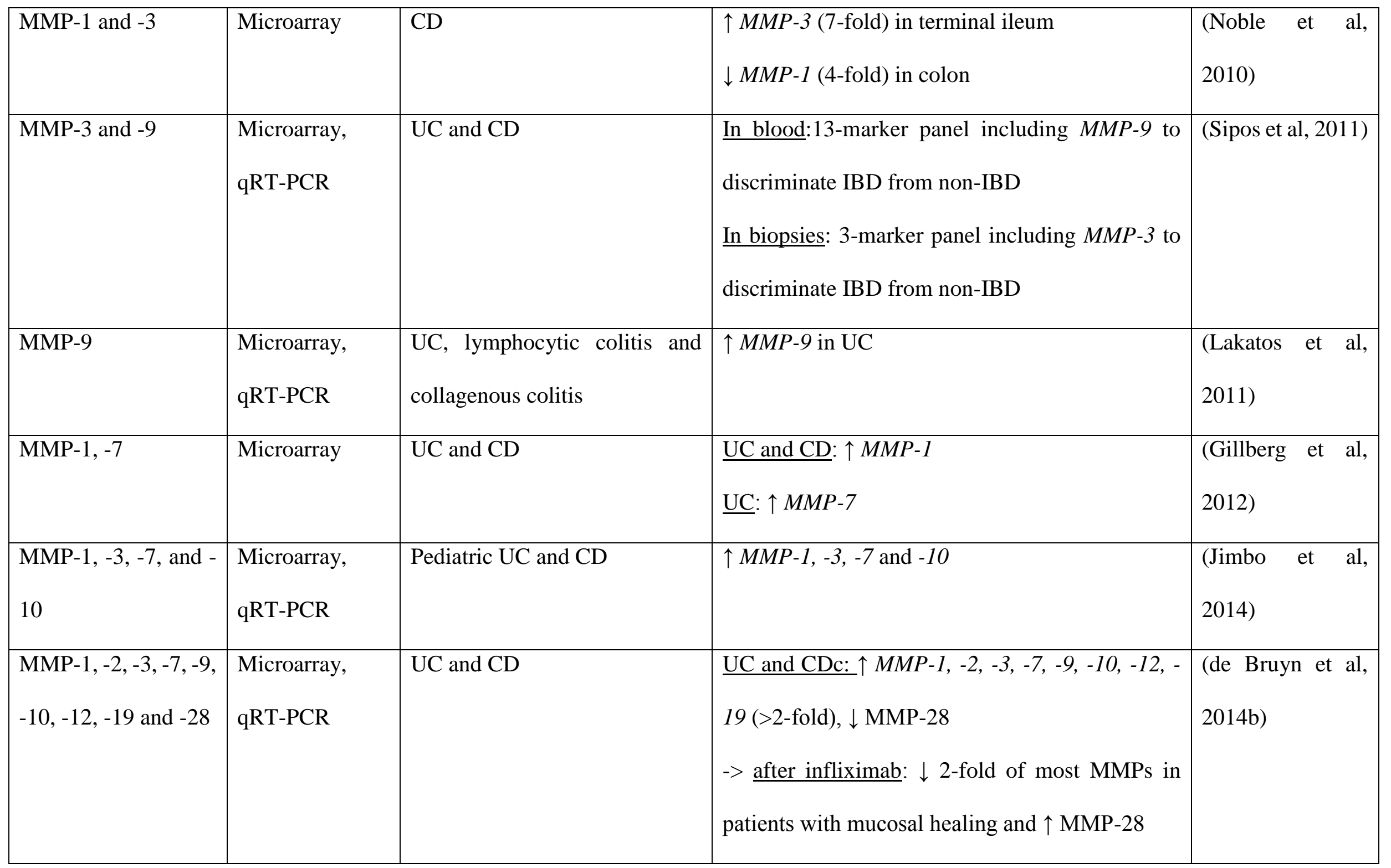




\begin{tabular}{|c|c|c|c|c|}
\hline & & & $\begin{array}{l}\text { CDi: } \uparrow M M P-1,-3,-7 \text { and }-10 \\
->\text { after infliximab: } \downarrow \text { 2-fold of MMPs in patients } \\
\text { with mucosal healing }\end{array}$ & \\
\hline \multicolumn{5}{|c|}{ Targeted mRNA expression studies } \\
\hline MMP-1, $-2,-3,-14$ & qRT-PCR & $\mathrm{UC}$ and $\mathrm{CD}$ & $\begin{array}{l}\uparrow M M P-1 \text { and }-3 \text { (15-fold) } \\
\uparrow \quad M M P-2 \text { and }-14 \text { in inflamed mucosa } \\
\text { (marginally) and in ulcerated mucosa (9-12-fold) }\end{array}$ & $\begin{array}{l}\text { (von Lampe et al, } \\
\text { 2000) }\end{array}$ \\
\hline MMP-3 & qRT-PCR & Pediatric UC and CD & $\begin{array}{l}\uparrow M M P-3 \text { in inflamed tissue and } \downarrow \text { after enteral } \\
\text { nutrition }\end{array}$ & $\begin{array}{l}\text { (Heuschkel et al, } \\
\text { 2000) }\end{array}$ \\
\hline MMP-2 and -9 & qRT-PCR & $\mathrm{UC}$ and $\mathrm{CD}$ & $\begin{array}{l}\uparrow M M P-2 \text { (2-fold), but no difference between } \\
\text { inflamed or non-inflamed } \\
\uparrow M M P-9 \text { in non-inflamed tissue (2-fold) and in } \\
\text { inflamed tissue (4-fold) }\end{array}$ & (Gao et al, 2005) \\
\hline
\end{tabular}




\begin{tabular}{|c|c|c|c|c|}
\hline MMP-1 & qRT-PCR & UC & $\begin{array}{l}\uparrow M M P-1 \text { and correlation with severity clinical } \\
\text { symptoms }\end{array}$ & $\begin{array}{l}\text { (Wang \& Yan, } \\
\text { 2006) }\end{array}$ \\
\hline MMP-1 & qRT-PCR & UC & $\uparrow M M P-1$ and correlation with disease severity & $\begin{array}{l}\text { (Wang \& Mao, } \\
\text { 2007) }\end{array}$ \\
\hline MMP-1 & qRT-PCR & UC and pouchitis & $\uparrow M M P-1$ in pouchitis & $\begin{array}{l}\text { (Schmidt et al, } \\
\text { 2007) }\end{array}$ \\
\hline MMP-2 & SSH, qRT-PCR & Pediatric ileal CD & $\uparrow M M P-2$ & (Sim et al, 2012) \\
\hline MMP-7 & qRT-PCR & $\mathrm{UC}$ and $\mathrm{CD}$ & $\begin{array}{l}M M P-7 \text { does not discriminate between } \mathrm{UC} \text { and } \\
\mathrm{CD}\end{array}$ & $\begin{array}{l}\text { (Bjerrum et al, } \\
\text { 2014) }\end{array}$ \\
\hline IRNA loca & tion studies & & & \\
\hline
\end{tabular}




\begin{tabular}{|c|c|c|c|c|}
\hline MMP-1, -3, - 7 & ISH & $\begin{array}{l}\mathrm{UC} \text { and } \mathrm{CD} \text {, gastrointestinal } \\
\text { ulcers }\end{array}$ & $\begin{array}{l}M M P-1 \text { and }-3: \text { granulation tissue } \\
M M P-7: \text { epithelium at ulcer }\end{array}$ & $\begin{array}{l}\text { (Saarialho-Kere } \\
\text { et al, 1996) }\end{array}$ \\
\hline MMP-1, -2 & ISH & UC and pouchitis & $\uparrow M M P-1$ and $-2:$ myofibroblasts & $\begin{array}{l}\text { (Stallmach et al, } \\
\text { 2000) }\end{array}$ \\
\hline $\begin{array}{l}\text { MMP-1, -2, -3, } \\
-7,-9,-10\end{array}$ & ISH & $\mathrm{CD}$ & $\begin{array}{l}\uparrow M M P-3 \text { : mononuclear cells and fibroblasts in } \\
\text { fistula } \\
\uparrow M M P-9 \text { : granulocytes in fistula } \\
=M M P-2 \text { : endothelial cells and macrophages }\end{array}$ & $\begin{array}{l}\text { (Kirkegaard et al, } \\
\text { 2004) }\end{array}$ \\
\hline
\end{tabular}

Abbreviations: ISH, in situ hybridization; CDc, colonic CD; CDi, ileal CD; PC, plasma cell; SSH, suppressive subtractive hybridization. Symbols:

$=$, unchanged; $\uparrow$, increased; $\downarrow$, decreased. 
Table 4: Overview of MMP protein expression studies in human samples.

\begin{tabular}{|c|c|c|c|c|c|}
\hline MMP & Method & IBD type & Sample & Main findings & References \\
\hline \multicolumn{6}{|c|}{ In vitro/ex vivo studies } \\
\hline $\begin{array}{l}\text { MMP-1,-2, -3, } \\
-9\end{array}$ & $\begin{array}{l}\text { ELISA, } \\
\text { zymograph } \\
\text { y, IHC }\end{array}$ & NA & $\begin{array}{l}\text { Culture of human } \\
\text { fetal small } \\
\text { intestine }\end{array}$ & $\begin{array}{l}\text { after T cell activation: } \\
\text { ELISA: } \uparrow \text { MMP-1 (3-fold) and MMP-3 (10-fold), = MMP-2, } \\
\text { MMP-9 (below limit) } \\
\text { WB: activated MMP-1, -3 and proMMP-9 } \\
\text { Zymography: } \uparrow \text { proMMP-9 and activated and latent MMP-2 } \\
\text { IHC: MMP-1 and }-3 \text { in subepithelial layer }\end{array}$ & $\begin{array}{l}\text { (Pender et al, } \\
1997)\end{array}$ \\
\hline $\begin{array}{l}\text { MMP-1, }-2,-3 \\
\text { and }-9\end{array}$ & WB & NA & $\begin{array}{l}\text { Organ culture of } \\
\text { human fetal small } \\
\text { intestine }\end{array}$ & 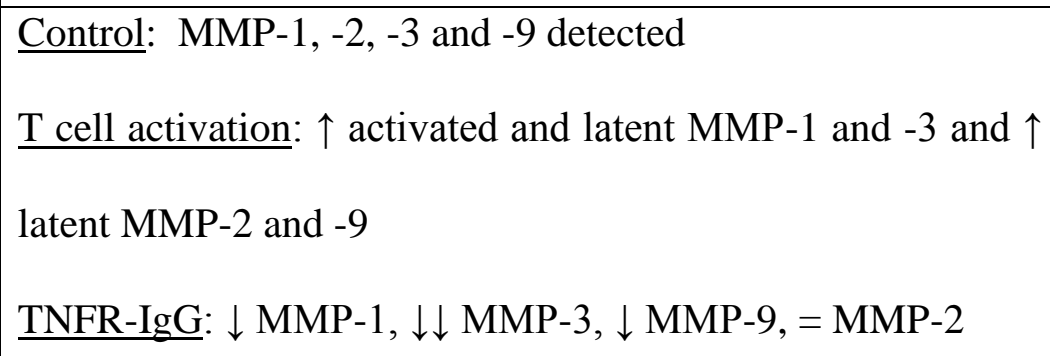 & $\begin{array}{l}\text { (Pender et al, } \\
1998)\end{array}$ \\
\hline
\end{tabular}




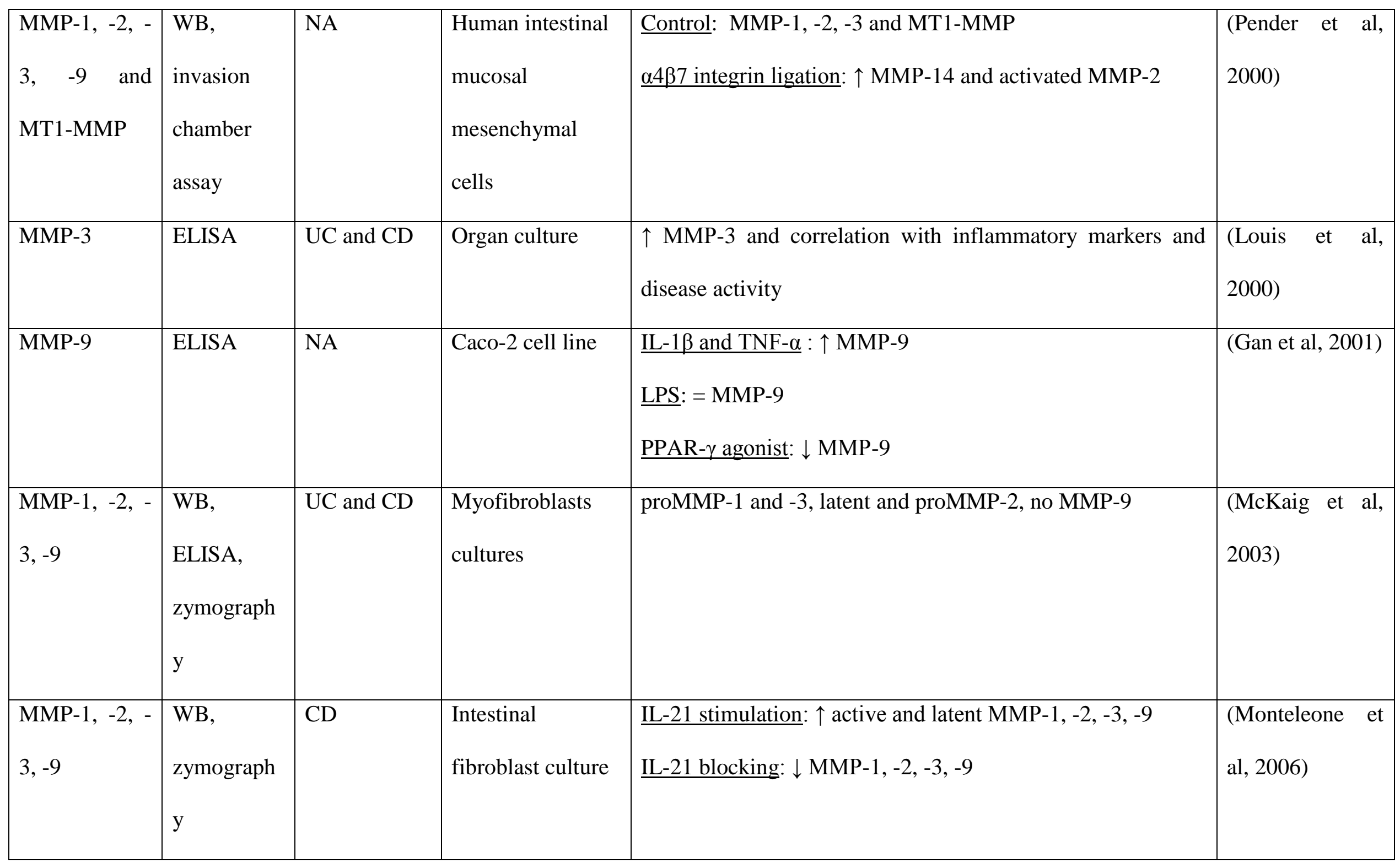




\begin{tabular}{|c|c|c|c|c|c|}
\hline $\begin{array}{l}\text { MMP-1, -2, - } \\
3,-9\end{array}$ & $\begin{array}{l}\text { ELISA, } \\
\text { bioactivity } \\
\text { assays }\end{array}$ & $\mathrm{UC}$ and $\mathrm{CD}$ & Intestinal explants & 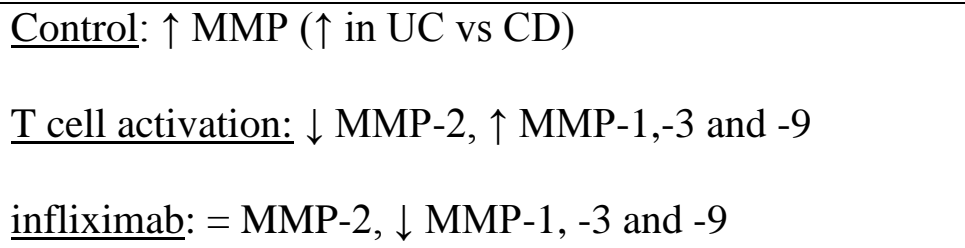 & $\begin{array}{l}\text { (Meijer et al, } \\
\text { 2007b) }\end{array}$ \\
\hline MMP-3 & $\begin{array}{l}\text { ELISA, } \\
\text { Western } \\
\text { blotting, } \\
\text { confocal } \\
\text { microscopy }\end{array}$ & $\mathrm{UC}$ and $\mathrm{CD}$ & $\begin{array}{l}\text { Gut PCs isolated } \\
\text { from colonic } \\
\text { tissue }\end{array}$ & $\uparrow$ MMP-3 in IgG positive PCs & $\begin{array}{l}\text { (Gordon et al, } \\
2008)\end{array}$ \\
\hline MMP-3 & WB & $\mathrm{UC}$ and $\mathrm{CD}$ & $\begin{array}{l}\text { Cultured gut } \\
\text { LPMCs and } \\
\text { myofibroblasts }\end{array}$ & PDE4: $\downarrow$ MMP-3 in LPMCs, but not in myofibroblasts & $\begin{array}{l}\text { (Gordon et al, } \\
2009 \text { ) }\end{array}$ \\
\hline MMP-2 & $\begin{array}{l}\text { WB, Ussing } \\
\text { chambers }\end{array}$ & NA & $\begin{array}{l}\text { Caco-2 epithelial } \\
\text { cells and } \\
\text { mesenchymal } \\
\text { 3T3 fibroblasts }\end{array}$ & Local inhibition of MMP-2 restores the epithelial barrier & $\begin{array}{l}\text { (Ailenberg \& } \\
\text { Sefton, 2009) }\end{array}$ \\
\hline
\end{tabular}




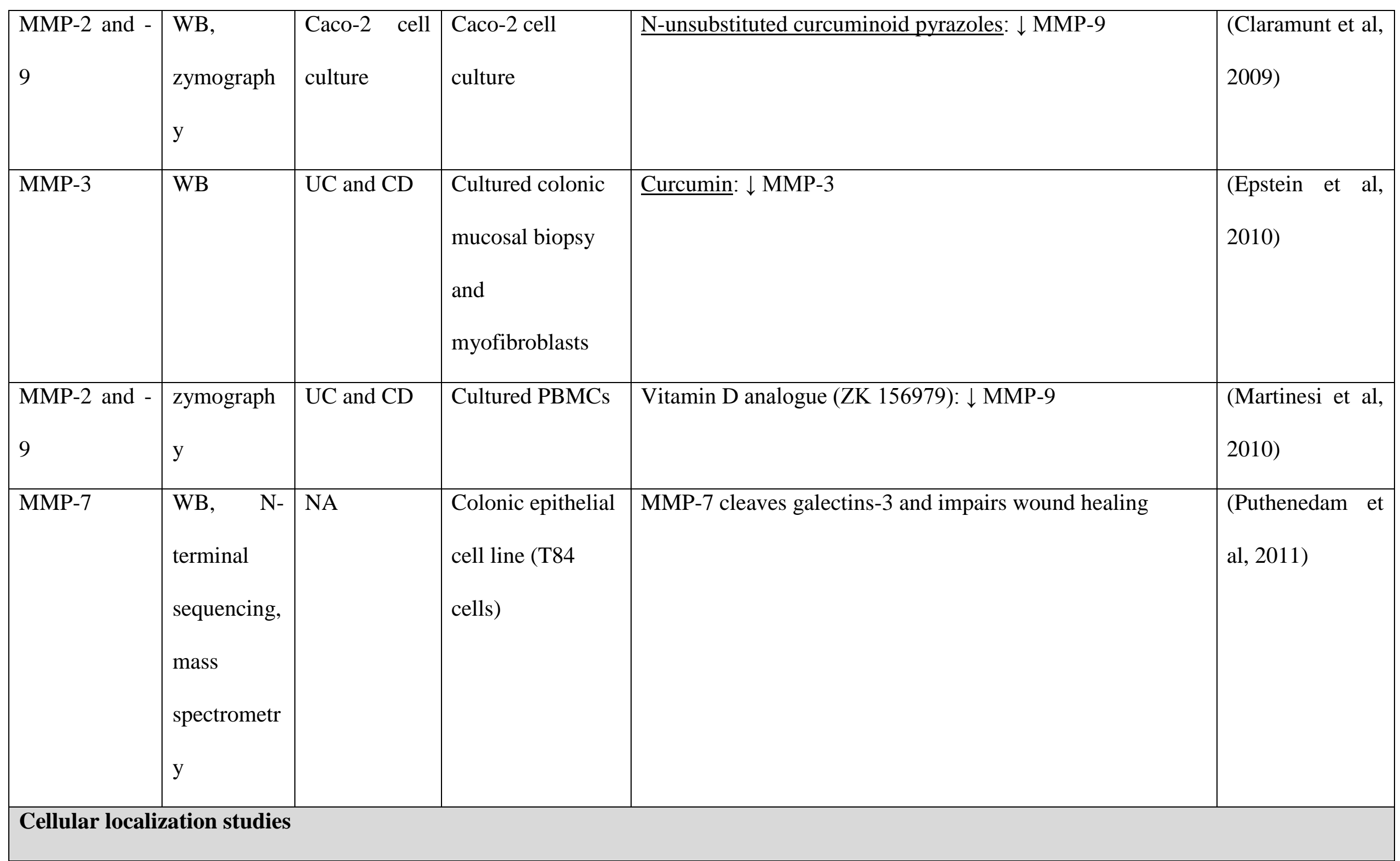




\begin{tabular}{|c|c|c|c|c|c|}
\hline $\begin{array}{l}\text { MMP-1, -2, - } \\
3,-9\end{array}$ & IF & $\mathrm{UC}$ and $\mathrm{CD}$ & Mucosal biopsy & $\begin{array}{l}\text { Inflamed tissue: } \\
\text { MMP-1: mononuclear cells } \\
\text { MMP-2: no signal } \\
\text { MMP-9: PMNL in lamina propria, submucosa and muscularis } \\
\text { propria } \\
\text { MMP-3: mononuclear cells }\end{array}$ & $\begin{array}{l}\text { (Bailey et al, } \\
\text { 1994) }\end{array}$ \\
\hline MMP- 7 & IHC & $\begin{array}{l}\text { UC and CD, } \\
\text { gastrointestin } \\
\text { al ulcers }\end{array}$ & Mucosal biopsy & $\begin{array}{l}\text { Epithelium bordering neutrophil-associated disrupted areas - } \\
>\text { involved in epithelial regeneration }\end{array}$ & $\begin{array}{l}\text { (Saarialho-Kere } \\
\text { et al, 1996) }\end{array}$ \\
\hline MMP-10 & IHC & $\mathrm{UC}$ and $\mathrm{CD}$ & Mucosal biopsy & Epithelium at ulcer -> involved in epithelial migration & $\begin{array}{l}\text { (Vaalamo et al, } \\
\text { 1998) }\end{array}$ \\
\hline MMP -9 & IF & $\begin{array}{l}\text { Pediatric and } \\
\text { adult UC and } \\
\text { CD }\end{array}$ & Mucosal biopsy & Neutrophils in lamina propria & $\begin{array}{l}\text { (Baugh et al, } \\
\text { 1999) }\end{array}$ \\
\hline MMP-1, -3 & IHC & $\mathrm{UC}$ and $\mathrm{CD}$ & Mucosal biopsy & $\begin{array}{l}\text { MMP-1: macrophages, endothelial cells, and cell aggregates } \\
\text { in severely inflamed tissue }\end{array}$ & $\begin{array}{l}\text { (von Lampe et } \\
\text { al, 2000) }\end{array}$ \\
\hline
\end{tabular}




\begin{tabular}{|c|c|c|c|c|c|}
\hline & & & & MMP-3: macrophages & \\
\hline $\begin{array}{l}\text { MMP-1, }-3,-8 \\
\text { and }-9\end{array}$ & IHC & $\mathrm{UC}$ and $\mathrm{CD}$ & Mucosal biopsy & $\begin{array}{l}\text { MMP-1, }-3,-8 \text { and }-9 \text { : Inflammatory cells and fibroblasts at } \\
\text { ulcer bases } \\
\text { MMP-1 and -9: vascular smooth muscle cells }\end{array}$ & $\begin{array}{l}\text { (Arihiro et al, } \\
\text { 2001) }\end{array}$ \\
\hline $\begin{array}{l}\text { MMP-1, -2, - } \\
3,-7,-9\end{array}$ & IHC & UC & Mucosal biopsy & $\begin{array}{l}\text { MMP-1, -2 and -9: stromal cells } \\
\text { MMP-3: epithelial and stromal cells } \\
\text { MMP-7: epithelial cells at ulcer edge and correlated with } \\
\text { disease severity. }\end{array}$ & $\begin{array}{l}\text { (Matsuno et al, } \\
\text { 2003) }\end{array}$ \\
\hline $\begin{array}{l}\text { MMP-1, -2, - } \\
3,-7,-9,-10\end{array}$ & IHC & $\mathrm{CD}$ & Mucosal biopsy & $\begin{array}{l}\text { MMP-3: large mononuclear cells and fibroblasts } \\
\text { MMP-9: granulocytes }\end{array}$ & $\begin{array}{l}\text { (Kirkegaard et } \\
\text { al, 2004) }\end{array}$ \\
\hline $\begin{array}{l}\text { MMP-19, }-26 \text {, } \\
\text { and }-28\end{array}$ & IHC & $\mathrm{UC}$ and $\mathrm{CD}$ & Mucosal biopsy & $\begin{array}{l}\text { MMP-19: non-migrating enterocytes and shedding epithelium } \\
\text { MMP-26: migrating enterocytes } \\
\text { MMP-28: not associated }\end{array}$ & $\begin{array}{l}\text { (Bister et al, } \\
\text { 2004) }\end{array}$ \\
\hline MMP-9 & IHC & $\mathrm{CD}$ & Mucosal biopsy & MMP-9 $\downarrow$ after infliximab & $\begin{array}{l}\text { (Geboes et al, } \\
2005)\end{array}$ \\
\hline
\end{tabular}




\begin{tabular}{|c|c|c|c|c|c|}
\hline $\begin{array}{l}\text { MMP-2 and - } \\
9\end{array}$ & IHC & $\mathrm{UC}$ and $\mathrm{CD}$ & Mucosal biopsy & $\begin{array}{l}\text { MMP-2: submucosa and some epithelial cells } \\
\text { MMP-9: PMNL }\end{array}$ & (Gao et al, 2005) \\
\hline $\begin{array}{l}\text { MMP-1 and - } \\
13\end{array}$ & IHC & $\mathrm{CD}$ & Mucosal biopsy & $\begin{array}{l}\text { MMP-1: mucosal glands and subepithelial } \\
\text { MMP-13: epithelial basement membrane and in stroma }\end{array}$ & $\begin{array}{l}\text { (Stumpf et al, } \\
2005)\end{array}$ \\
\hline MMP-13 & IHC & $\mathrm{UC}$ and $\mathrm{CD}$ & Mucosal biopsy & Ulcerated mucosa & $\begin{array}{l}\text { (Vizoso et al, } \\
\text { 2006) }\end{array}$ \\
\hline $\begin{array}{l}\text { MMP-2 and - } \\
9\end{array}$ & IHC & $\mathrm{CD}$ & $\begin{array}{l}\text { Serum and } \\
\text { mucosal biopsy }\end{array}$ & MMP-2 $=$ and MMP-9 $\downarrow$ after infliximab & (Gao et al, 2007) \\
\hline $\begin{array}{l}\text { MMP-1, -7, - } \\
9,- \\
10, \text { and }-26\end{array}$ & IHC & $\mathrm{CD}$ & Mucosal biopsy & MMP-7, -9 and -26 $\downarrow$ after immunosuppressive treatment. & $\begin{array}{l}\text { (Makitalo et al, } \\
\text { 2009) }\end{array}$ \\
\hline $\begin{array}{l}\text { MMP-7, 13, - } \\
28\end{array}$ & IHC & UC & Mucosal biopsy & $\begin{array}{l}\text { MMP-7 and -13: inflammatory and endothelial cells } \\
\text { MMP-28: } \downarrow \text { and linked with epithelial destruction and loss of } \\
\text { crypt architecture }\end{array}$ & (Rath et al, 2010) \\
\hline
\end{tabular}




\begin{tabular}{|c|c|c|c|c|c|}
\hline $\begin{array}{l}\text { MMP-1, }-7,- \\
8,-9,-10,-12 \\
\text { and }-26\end{array}$ & IHC & $\begin{array}{l}\text { Pediatric UC } \\
\text { and } \mathrm{CD}\end{array}$ & Mucosal biopsy & $\begin{array}{l}\text { MMP-7: epithelium ( } \uparrow \text { in CD vs UC) }->\text { differentiation UC } \\
\text { and CD colitis } \\
\text { MMP-10: epithelium -> histological indicator of IBD etiology }\end{array}$ & $\begin{array}{l}\text { (Makitalo et al, } \\
\text { 2010) }\end{array}$ \\
\hline MMP-9 & IHC & $\mathrm{UC}$ and $\mathrm{CD}$ & $\begin{array}{l}\text { Blood smears and } \\
\text { mucosal biopsies }\end{array}$ & $\uparrow$ MMP-9 positive cells & $\begin{array}{l}\text { (Sipos et al, } \\
2011)\end{array}$ \\
\hline MMP-9 & IHC & $\begin{array}{l}\text { UC, } \\
\text { lymphocytic } \\
\text { colitis and } \\
\text { collagenous } \\
\text { colitis }\end{array}$ & Mucosal biopsy & PMNL in submucosa and occasionally macrophages & $\begin{array}{l}\text { (Lakatos et al, } \\
\text { 2011) }\end{array}$ \\
\hline $\begin{array}{l}\text { MMPs-3, -7, - } \\
8,-9,-12 \text { and - } \\
26\end{array}$ & IHC & $\begin{array}{l}\text { Pediatric UC } \\
\text { pouch }\end{array}$ & Ileal pouch biopsy & $\begin{array}{l}\text { MMP-3 }(\uparrow),-7(\uparrow) \text { and }-12: \text { epithelial and stromal cells } \\
\text { MMP-8, -9 and -26: not detected }\end{array}$ & $\begin{array}{l}\text { (Makitalo et al, } \\
\text { 2012a) }\end{array}$ \\
\hline MMP-9 & IHC & $\mathrm{UC}$ & Mucosal biopsy & Stromal inflammatory cells & $\begin{array}{l}\text { (Piekkala et al, } \\
\text { 2013) }\end{array}$ \\
\hline
\end{tabular}




\begin{tabular}{|c|c|c|c|c|c|}
\hline $\begin{array}{l}\text { MMP-1, -3, - } \\
7, \text { and }-10\end{array}$ & $\mathrm{IHC}$ & $\begin{array}{l}\text { Pediatric UC } \\
\text { and } \mathrm{CD}\end{array}$ & Mucosal biopsy & MMP-1, $-3,-7$, and -10 in epithelial cells and lamina propria & $\begin{array}{l}\text { (Jimbo et al, } \\
2014)\end{array}$ \\
\hline MMP-1 & IHC & UC and CD & Mucosal biopsy & $\begin{array}{l}\text { Normal: primitive cells at the base of the crypts } \\
\text { Inflamed: immature and surface epithelium, endothelial cells }\end{array}$ & $\begin{array}{l}\text { (de Bruyn et al, } \\
2014 b \text { ) }\end{array}$ \\
\hline \multicolumn{6}{|c|}{ Quantification studies } \\
\hline $\begin{array}{l}\text { MMP-1, }-2,-3 \\
\text { and }-9\end{array}$ & $\begin{array}{l}\text { Zymograph } \\
\text { y, WB }\end{array}$ & $\begin{array}{l}\text { Pediatric and } \\
\text { adult UC and } \\
\text { CD }\end{array}$ & Mucosal biopsy & $\begin{array}{l}\text { Zymography: } \uparrow \text { proteolytic activity in UC, not in CD } \\
\underline{\text { WB: }} \uparrow \text { activated and latent MMP-9, activated MMP-3 }\end{array}$ & $\begin{array}{l}\text { (Baugh et al, } \\
\text { 1999) }\end{array}$ \\
\hline MMP-1, -2, -3 & WB & $\mathrm{UC}$ and $\mathrm{CD}$ & Mucosal biopsy & $\uparrow$ MMP-1, -2 and -3 & $\begin{array}{l}\text { (von Lampe et } \\
\text { al, 2000) }\end{array}$ \\
\hline MMP-3 & WB & $\begin{array}{l}\text { Pediatric UC } \\
\text { and } \mathrm{CD}\end{array}$ & Mucosal biopsy & $\uparrow$ MMP-3 & $\begin{array}{l}\text { (Heuschkel et al, } \\
\text { 2000) }\end{array}$ \\
\hline MMP-1, -2 & $\begin{array}{l}\text { WB, } \\
\text { ELISA }\end{array}$ & $\begin{array}{l}\text { UC and } \\
\text { pouchitis }\end{array}$ & Mucosal biopsy & $\uparrow \mathrm{MMP}-1$ and -2 & $\begin{array}{l}\text { (Stallmach et al, } \\
\text { 2000) }\end{array}$ \\
\hline
\end{tabular}




\begin{tabular}{|c|c|c|c|c|c|}
\hline MMP-1 & ELISA & UC & Plasma & $\uparrow \mathrm{MMP}-1$ & $\begin{array}{l}\text { (Wiercinska- } \\
\text { Drapalo et al, } \\
\text { 2003) }\end{array}$ \\
\hline MMP-2, -9 & $\begin{array}{l}\text { IHC, } \\
\text { zymograph } \\
\text { y }\end{array}$ & $\mathrm{CD}$ & Mucosal biopsy & $\uparrow$ MMP-3, activated MMP-2 and -9 in fistula & $\begin{array}{l}\text { (Kirkegaard et } \\
\text { al, 2004) }\end{array}$ \\
\hline $\begin{array}{l}\text { MMP-2 and - } \\
9\end{array}$ & $\begin{array}{l}\text { ELISA, } \\
\text { zymograph } \\
\mathrm{y}, \quad \text { activity } \\
\text { test }\end{array}$ & $\mathrm{UC}$ and $\mathrm{CD}$ & Mucosal biopsy & $\begin{array}{l}\text { ELISA }: \uparrow \text { MMP-2 and -9 } \\
\text { Zymography: } \uparrow \text { latent and activated MMP-2 and }-9 \\
\underline{\text { Activity test: }}=\text { MMP-2, } \uparrow \text { MMP-9 }\end{array}$ & (Gao et al, 2005) \\
\hline MMP-2, -9 & ELISA & $\mathrm{UC}$ and $\mathrm{CD}$ & Mucosal biopsy & $\uparrow$ MMP-2 and -9 & (Rath et al, 2006) \\
\hline MMP-2, -9 & $\begin{array}{l}\text { Zymograph } \\
\text { y, WB }\end{array}$ & $\begin{array}{l}\text { Ischemic } \\
\text { colitis }\end{array}$ & Mucosal biopsy & $\uparrow$ MMP-2 and -9 & $\begin{array}{l}\text { (Medina et al, } \\
\text { 2006b) }\end{array}$ \\
\hline $\begin{array}{l}\text { MMP-1, -2, - } \\
3,-9\end{array}$ & $\begin{array}{l}\text { ELISA, } \\
\text { specific }\end{array}$ & $\mathrm{UC}$ and $\mathrm{CD}$ & Mucosal biopsy & $\uparrow$ MMP-1, $-2,-3$ and -9 & $\begin{array}{l}\text { (Meijer et al, } \\
\text { 2007a) }\end{array}$ \\
\hline
\end{tabular}




\begin{tabular}{|c|c|c|c|c|c|}
\hline & activity & & & & \\
\hline $\begin{array}{l}\text { MMP-2 and - } \\
9\end{array}$ & ELISA & $\mathrm{CD}$ & $\begin{array}{l}\text { Serum and } \\
\text { mucosal biopsies }\end{array}$ & $\underline{\text { after infliximab: }}: \uparrow$ MMP- $2, \downarrow$ MMP-9 & (Gao et al, 2007) \\
\hline $\begin{array}{l}\text { MMP-2 and - } \\
9\end{array}$ & $\begin{array}{l}\text { zymograph } \\
\text { y }\end{array}$ & Pediatric IBD & Urine & $\uparrow$ MMP-2, MMP-9 and NGAL-MMP-9 & $\begin{array}{l}\text { (Manfredi et al, } \\
\text { 2008) }\end{array}$ \\
\hline MMP-7, -13 & ELISA & $\mathrm{UC}$ & Mucosal biopsy & $\uparrow$ MMP-7, undetectable MMP-13 & (Rath et al, 2010) \\
\hline MMP-9 & ELISA & $\begin{array}{l}\text { UC, } \\
\text { lymphocytic } \\
\text { colitis and } \\
\text { collagenous } \\
\text { colitis }\end{array}$ & Mucosal biopsy & $\uparrow$ MMP-9 in UC & $\begin{array}{l}\text { (Lakatos et al, } \\
\text { 2011) }\end{array}$ \\
\hline MMP-7, $-8,-9$ & ELISA & $\begin{array}{l}\text { Pediatric UC } \\
\text { and } \mathrm{CD}\end{array}$ & Serum & $\begin{array}{l}\uparrow \text { MMP-7, -8 and -9 } \\
\text { After GC or anti-TNF: } \downarrow \text { MMP-7 }\end{array}$ & $\begin{array}{l}\text { (Makitalo et al, } \\
\text { 2012b) }\end{array}$ \\
\hline MMP-9 & ELISA & $\mathrm{UC}$ & Feces & $\begin{array}{l}\uparrow \text { MMP-9 and correlation with clinical, endoscopic and } \\
\text { inflammatory markers }\end{array}$ & $\begin{array}{l}\text { (Annahazi et al, } \\
\text { 2013) }\end{array}$ \\
\hline
\end{tabular}




\begin{tabular}{|c|c|c|c|c|c|}
\hline MMP-9 & ELISA & $\mathrm{UC}$ and $\mathrm{CD}$ & Serum & $\begin{array}{l}\text { Combination serum MMP-9 and fecal calprotectin best for } \\
\text { discrimination UC and CD }\end{array}$ & $\begin{array}{l}\text { (Faubion et al, } \\
\text { 2013) }\end{array}$ \\
\hline $\begin{array}{l}\text { MMP-3 and - } \\
9\end{array}$ & ELISA & Pediatric UC & Serum & $\uparrow$ MMP-3 and -9 & $\begin{array}{l}\text { (Kofla-Dlubacz } \\
\text { et al, 2014) }\end{array}$ \\
\hline $\begin{array}{l}\text { MMP-2, }-3 \\
\text { and }-9\end{array}$ & $\begin{array}{l}\text { WB, } \\
\text { zymograph }\end{array}$ & $\mathrm{UC}$ and $\mathrm{CD}$ & $\begin{array}{l}\text { Mucosal biopsy } \\
\text { and PBMCs }\end{array}$ & 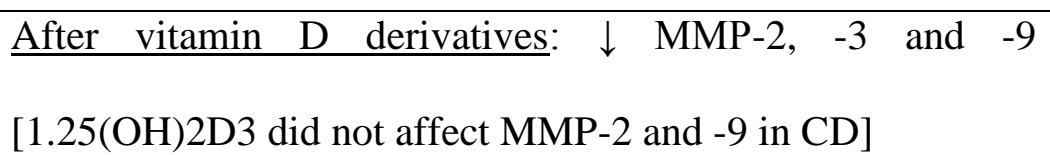 & $\begin{array}{l}\text { (Martinesi et al, } \\
\text { 2014) }\end{array}$ \\
\hline MMP-9 & ELISA & Pediatric IBD & Feces & $\begin{array}{l}\uparrow \text { MMP-9 with comparable performance than fecal } \\
\text { calprotectin }\end{array}$ & $\begin{array}{l}\text { (Kolho et al, } \\
\text { 2014) }\end{array}$ \\
\hline MMP-2, -9 & $\begin{array}{l}\text { Zymograph } \\
\text { y and } \\
\text { gelatin } \\
\text { degradation } \\
\text { assay }\end{array}$ & $\mathrm{UC}$ and $\mathrm{CD}$ & Mucosal biopsy & $\begin{array}{l}\uparrow \text { MMP-9 and NGAL-MMP-9 in inflamed biopsies }-\downarrow \text { after } \\
\text { infliximab } \\
\uparrow \text { gelatinolytic activity in inflamed biopsies }-\downarrow \text { after } \\
\text { infliximab }\end{array}$ & $\begin{array}{l}\text { (de Bruyn et al, } \\
\text { 2014b) }\end{array}$ \\
\hline
\end{tabular}




\begin{tabular}{|c|c|c|c|c|c|}
\hline $\begin{array}{l}\text { MMP-2 and - } \\
9, \quad \text { NGAL- } \\
\text { MMP-9 } \\
\text { complex }\end{array}$ & \begin{tabular}{|l} 
Zymograph \\
y and \\
ELISA
\end{tabular} & $\mathrm{UC}$ & Serum & $\begin{array}{l}\uparrow \text { MMP-9 and NGAL-MMP-9 } \\
\downarrow \text { after infliximab in patients with mucosal healing }\end{array}$ & $\begin{array}{l}\text { (de Bruyn et al, } \\
\text { 2014a) }\end{array}$ \\
\hline $\begin{array}{l}\text { MMP-3 and - } \\
9\end{array}$ & ELISA & $\mathrm{UC}$ and $\mathrm{CD}$ & Serum & $\uparrow$ MMP-3 and -9 & $\begin{array}{l}\text { (Silosi et al, } \\
\text { 2014) }\end{array}$ \\
\hline MMP-9 & ELISA & $\mathrm{UC}$ and $\mathrm{CD}$ & Feces & $\uparrow$ MMP-9 and correlation with UC disease activity, not CD & $\begin{array}{l}\text { (Farkas et al, } \\
\text { 2015) }\end{array}$ \\
\hline $\begin{array}{l}\text { NGAL-MMP- } \\
9 \text { complex }\end{array}$ & ELISA & $\mathrm{CD}$ & Serum & $\begin{array}{l}\uparrow \text { NGAL-MMP-9 } \\
\downarrow \text { after infliximab in patients with mucosal healing }\end{array}$ & $\begin{array}{l}\text { (de Bruyn et al, } \\
\text { 2015) }\end{array}$ \\
\hline
\end{tabular}

Abbreviations: ELISA, enzyme-linked immunosorbent assay; GC, glucocorticoid; IHC, immunohistochemistry; PBMC, peripheral blood mononuclear cell; PC, plasma cell; LPMC, lamina propria mononuclear cell; NA, not applicable; WB, Western blot. Symbols: =, unchanged; $\uparrow$, increased; $\downarrow$, decreased, $\downarrow \downarrow$, strongly decreased. 
Table 5: Overview of TIMP mRNA expression studies in IBD.

\begin{tabular}{|c|c|c|c|c|}
\hline TIMP & Method & Sample/IBD type & Main findings & References \\
\hline TIMP-1 and -2 & qRT-PCR & $\begin{array}{l}\text { Myofibroblasts cultures and } \\
\text { mucosal samples (UC and CD) }\end{array}$ & $\uparrow \uparrow$ TIMP-1 & (McKaig et al, 2003) \\
\hline \multicolumn{5}{|c|}{ Genome-wide expression studies } \\
\hline
\end{tabular}




\begin{tabular}{|c|c|c|c|c|}
\hline TIMP-1 & Microarray & $\mathrm{UC}$ an $\mathrm{CD}$ & $=$ TIMP-1 & $\begin{array}{l}\text { (Lawrance et al, } \\
\text { 2001) }\end{array}$ \\
\hline TIMP-1 & Microarray & $\mathrm{UC}$ and $\mathrm{CD}$ & $\uparrow$ TIMP-1 & (Gillberg et al, 2012) \\
\hline TIMP-1, -2 & $\begin{array}{l}\text { Microarray, } \\
\text { qRT-PCR }\end{array}$ & $\mathrm{UC}$ and $\mathrm{CD}$ & $\begin{array}{l}\text { UC and CDc: } \uparrow \text { TIMP-1, }-2 \text { and MMP-1/TIMP-1 } \\
-\downarrow \text { after infliximab } \\
\text { CDi: TIMP- } 1-\downarrow \text { after infliximab }\end{array}$ & $\begin{array}{l}\text { (de Bruyn et al, } \\
\text { 2014b) }\end{array}$ \\
\hline \multicolumn{5}{|c|}{ Targeted expression studies } \\
\hline $\begin{array}{l}\text { TIMP-1 and } \\
\text { TIMP-2 }\end{array}$ & qRT-PCR & $\mathrm{UC}$ and $\mathrm{CD}$ & $\begin{array}{l}\uparrow \text { TIMP-1 in inflamed, } \uparrow \text { 9-12-fold TIMP-1 in } \\
\text { ulcerated tissue }\end{array}$ & $\begin{array}{l}\text { (von Lampe et al, } \\
\text { 2000) }\end{array}$ \\
\hline TIMP-1 & qRT-PCR & Pediatric UC and CD & $=\mathrm{TIMP}-1$ & $\begin{array}{l}\text { (Heuschkel et al, } \\
2000)\end{array}$ \\
\hline
\end{tabular}




\begin{tabular}{|l|l|l|l|l|l|}
\hline TIMP-1 & qRT-PCR & UC an CD & $\uparrow$ TIMP-1 & (Lawrance et al, \\
\hline TIMP-1 & qRT-PCR & UC & & (Wang \& Yan, 2006) \\
\hline TIMP-1 & qRT-PCR & UC and CD & TIMP-1 TIMP-1 & (Rath et al, 2006) \\
\hline TIMP-1 & qRT-PCR & UC and CD & TIMP-1 & (Leon et al, 2009) \\
\hline Cellular localization studies & ISH & UC and CD, gastrointestinal ulcers & Granulation tissue at base of the ulcer & (Saarialho-Kere et al, \\
\hline TIMP-1 & & & & Endothelial cells and macrophage- or fibroblast- & (Vaalamo et al, 1998) \\
\hline TIMP-3 & ISH & UC and CD & like cells / $\downarrow$ in inflamed tissue & \\
\hline
\end{tabular}

Abbreviations: ISH, in situ hybridization. Symbols: =, unchanged; $\uparrow$, increased; $\downarrow$, decreased. 
Table 6: Overview of TIMP protein expression studies.

\begin{tabular}{|c|c|c|c|c|c|}
\hline TIMP & Method & IBD type & Sample & Main findings & References \\
\hline \multicolumn{6}{|c|}{ In vitro/ex vivo studies } \\
\hline TIMP-1 and -2 & $\begin{array}{l}\text { ELISA and } \\
\text { WB }\end{array}$ & NA & $\begin{array}{l}\text { Culture of human } \\
\text { fetal small intestine }\end{array}$ & $=$ TIMP -1 and -2 after $\mathrm{T}$ cell stimulation & $\begin{array}{l}\text { (Pender et al, } \\
1997)\end{array}$ \\
\hline TIMP-1 & WB & NA & $\begin{array}{l}\text { Organ culture of } \\
\text { human fetal small } \\
\text { intestine and } \\
\text { mesenchymal cells }\end{array}$ & $\begin{array}{l}\text { Small intestine: } \\
\underline{\text { After T cell activation: } \uparrow \text { TIMP-1 }} \\
\underline{\text { After TNFR-IgG: }:=\text { TIMP-1 }} \\
\text { Mesenchymal cells: } \\
\underline{\text { TNF- } \alpha}: \uparrow \text { TIMP-1 } \\
\underline{\text { after TNFR-IgG: }: \downarrow \text { TIMP-1 }}\end{array}$ & $\begin{array}{l}\text { (Pender et al, } \\
1998)\end{array}$ \\
\hline TIMP-1 & ELISA & $\mathrm{UC}$ and $\mathrm{CD}$ & Organ culture & $\uparrow$ TIMP-1 & (Louis et al, 2000) \\
\hline TIMP-1 and -2 & ELISA & $\begin{array}{l}\mathrm{UC} \text { (active) and } \\
\mathrm{CD} \text { (fibrotic) }\end{array}$ & $\begin{array}{l}\text { Myofibroblasts } \\
\text { cultures and } \\
\text { mucosal samples }\end{array}$ & $\begin{array}{l}\uparrow \text { TIMP-1 in CD myofibroblasts compared with } \\
\text { normal or UC tissue } \\
\text { TIMP-2: no differences }\end{array}$ & $\begin{array}{l}\text { (McKaig et al, } \\
\text { 2003) }\end{array}$ \\
\hline
\end{tabular}




\begin{tabular}{|c|c|c|c|c|c|}
\hline TIMP-1 and -2 & WB & $\mathrm{CD}$ & $\begin{array}{l}\text { Intestinal } \\
\text { fibroblasts from } \\
\text { mucosal biopsies }\end{array}$ & IL-21 stimulation: no effect on TIMP-1 and -2 & $\begin{array}{l}\text { (Monteleone et al, } \\
\text { 2006) }\end{array}$ \\
\hline TIMP-1 and -2 & $\begin{array}{l}\text { ELISA, } \\
\text { bioactivity } \\
\text { assays }\end{array}$ & $\mathrm{UC}$ and $\mathrm{CD}$ & Intestinal explants & $\begin{array}{l}\uparrow \text { TIMP-1 } \\
=\text { TIMP-2 }\end{array}$ & $\begin{array}{l}\text { (Meijer et al, } \\
\text { 2007b) }\end{array}$ \\
\hline TIMP-1 & WB, ELISA & $\mathrm{UC}$ and $\mathrm{CD}$ & $\begin{array}{l}\text { Plasma cells } \\
\text { isolated from } \\
\text { colonic tissue }\end{array}$ & $=$ TIMP-1 & $\begin{array}{l}\text { (Gordon et al, } \\
\text { 2008) }\end{array}$ \\
\hline TIMP-1 & WB, ELISA & $\mathrm{UC}$ and $\mathrm{CD}$ & $\begin{array}{l}\text { Plasma cells } \\
\text { isolated from } \\
\text { colonic tissue }\end{array}$ & $=$ TIMP-1 & $\begin{array}{l}\text { (Gordon et al, } \\
\text { 2009) }\end{array}$ \\
\hline \multicolumn{6}{|c|}{ Cellular localization studies } \\
\hline TIMP-1 & IHC & $\mathrm{UC}$ and $\mathrm{CD}$ & Mucosal biopsy & $\begin{array}{l}\text { Activated fibroblasts and vascular smooth muscle } \\
\text { cells at the ulcer base }\end{array}$ & $\begin{array}{l}\text { (Arihiro et al, } \\
\text { 2001) }\end{array}$ \\
\hline
\end{tabular}




\begin{tabular}{|c|c|c|c|c|c|}
\hline TIMP-1, -2 & IHC & UC & Mucosal biopsy & Stromal cells, not epithelial cells & $\begin{array}{l}\text { (Matsuno et al, } \\
\text { 2003) }\end{array}$ \\
\hline $\begin{array}{l}\text { TIMP-1, TIMP-2, and } \\
\text { TIMP-3 }\end{array}$ & IHC & $\mathrm{CD}$ & Mucosal biopsy & $\begin{array}{l}\text { TIMP-1: lamina propria mononuclear cells } \\
\text { TIMP-2 and 3: colonic epithelium and lamina } \\
\text { propria mononuclear cells }\end{array}$ & $\begin{array}{l}\text { (Kirkegaard et al, } \\
\text { 2004) }\end{array}$ \\
\hline TIMP-1 and -3 & IHC & $\mathrm{CD}$ & Mucosal biopsy & $\begin{array}{l}\text { After immunosuppressive treatment: } \downarrow \text { stromal } \\
\text { TIMP-1 and }-3, \uparrow \text { epithelial TIMP-3 }\end{array}$ & $\begin{array}{l}\text { (Makitalo et al, } \\
\text { 2009) }\end{array}$ \\
\hline TIMP-1 and -3 & IHC & $\begin{array}{l}\text { Pediatric UC and } \\
\text { CD }\end{array}$ & Mucosal biopsy & $\uparrow$ stromal TIMP-3 & $\begin{array}{l}\text { (Makitalo et al, } \\
\text { 2010) }\end{array}$ \\
\hline TIMP-1, -2 and -3 & IHC & $\begin{array}{l}\text { Pediatric UC } \\
\text { pouch }\end{array}$ & Ileal pouch biopsy & Lack of TIMP-1, -2 and -3 in most pouch samples & $\begin{array}{l}\text { (Makitalo et al, } \\
\text { 2012a) }\end{array}$ \\
\hline TIMP-1 & IHC & $\mathrm{UC}$ and $\mathrm{CD}$ & Mucosal biopsy & $\begin{array}{l}\text { TIMP-1: enteroendocrine cells in both control } \\
\text { and inflamed tissue }\end{array}$ & $\begin{array}{l}\text { (de Bruyn et al, } \\
\text { 2014b) }\end{array}$ \\
\hline \multicolumn{6}{|l|}{ Quantification studies } \\
\hline TIMP-1 & WB & $\begin{array}{l}\text { Pediatric UC and } \\
\text { CD }\end{array}$ & Mucosal biopsy & No differences & $\begin{array}{l}\text { (Heuschkel et al, } \\
\text { 2000) }\end{array}$ \\
\hline
\end{tabular}




\begin{tabular}{|c|c|c|c|c|c|}
\hline TIMP-1 & ELISA & $\begin{array}{l}\mathrm{CD}, \mathrm{UC} \text { and } \\
\text { colon cancer }\end{array}$ & $\begin{array}{l}\text { Serum and EDTA } \\
\text { plasma }\end{array}$ & $\uparrow$ TIMP-1 only in colon cancer & $\begin{array}{l}\text { (Holten-Andersen } \\
\text { et al, 2002) }\end{array}$ \\
\hline TIMP-1 & ELISA & UC & Plasma & $\uparrow$ TIMP-1 & $\begin{array}{l}\text { (Wiercinska- } \\
\text { Drapalo et al, } \\
\text { 2003) }\end{array}$ \\
\hline TIMP-1 & ELISA & $\mathrm{UC}$ and $\mathrm{CD}$ & Mucosal biopsy & $\uparrow$ TIMP-1 only in UC & (Rath et al, 2006) \\
\hline TIMP-1, -2 and -4 & ELISA & $\mathrm{UC}$ and $\mathrm{CD}$ & Serum & $\begin{array}{l}\uparrow \text { TIMP-1 both UC and CD /higher TIMP-1 levels } \\
\text { in male vs female patients / lower TIMP-4 levels } \\
\text { in male vs female }\end{array}$ & $\begin{array}{l}\text { (Kapsoritakis et } \\
\text { al, 2008) }\end{array}$ \\
\hline TIMP-1, -2 & ELISA & $\begin{array}{l}\text { Pediatric UC and } \\
\text { CD }\end{array}$ & Serum & $\begin{array}{l}\text { After GC treatment: } \downarrow \text { TIMP-1 and MMP- } \\
\text { 7/TIMP-2 ratio }\end{array}$ & $\begin{array}{l}\text { (Makitalo et al, } \\
\text { 2012b) }\end{array}$ \\
\hline
\end{tabular}

Abbreviations: ELISA, enzyme-linked immunosorbent assay; GC, glucocorticoid; IHC, immunohistochemistry; PBMC, peripheral blood mononuclear cell; PC, plasma cell; LPMC, lamina propria mononuclear cell; NA, not applicable; WB, Western blot. Symbols: =, unchanged; $\uparrow$, increased; $\downarrow$, decreased. 
Table 7: Overview of MMP and TIMP animal association studies.

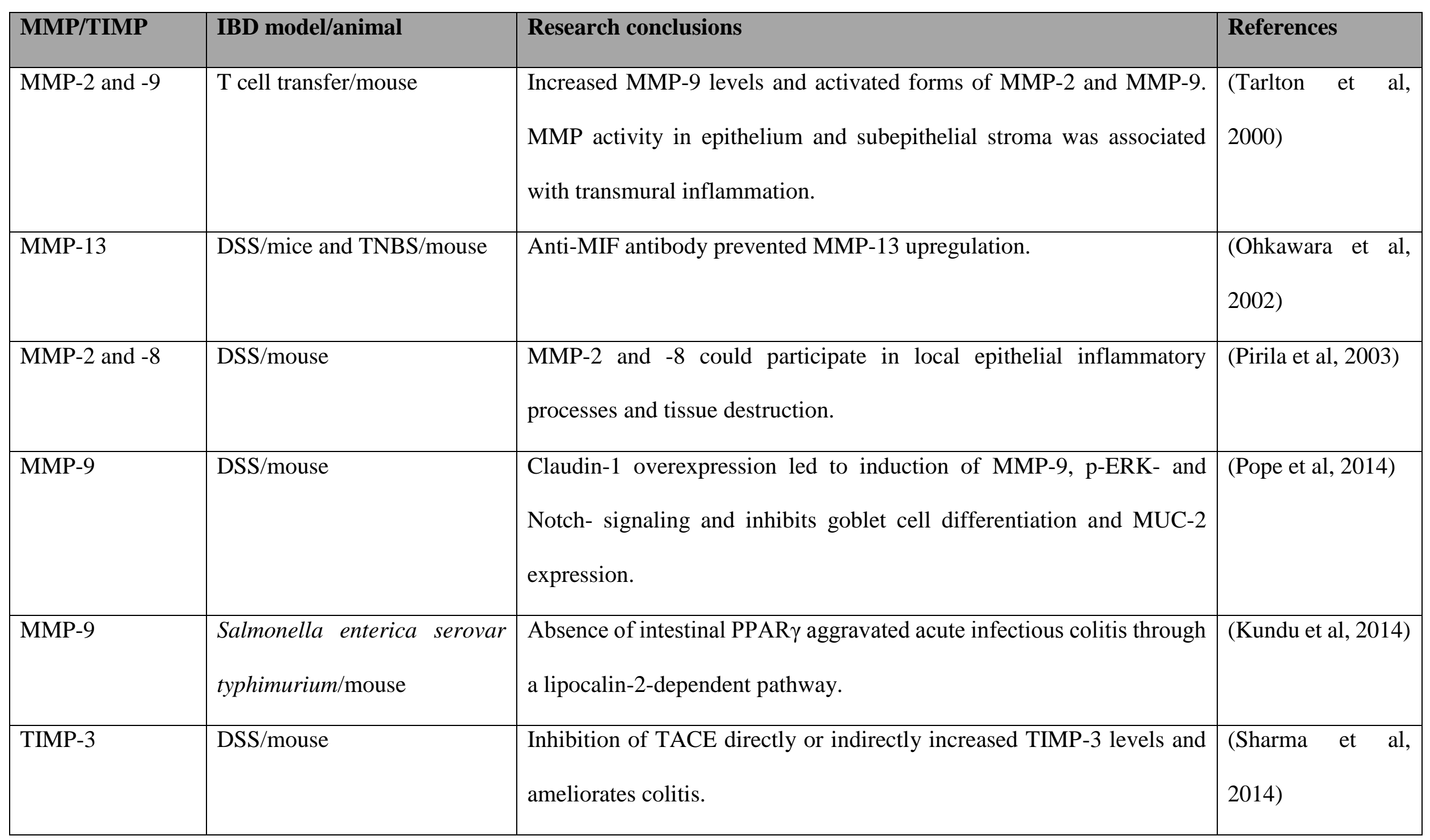




\begin{tabular}{|c|c|c|c|}
\hline MMP-9 & DSS/rat & Positive correlation between DAI, histological score and MMP-9 levels. & $\begin{array}{l}\text { (Oliveira et al, } \\
\text { 2014) }\end{array}$ \\
\hline $\begin{array}{l}\text { MMP-1 and } \\
\text { TIMP-1 }\end{array}$ & TNBS/mouse & Increased MMP-1 and TIMP-1 levels. & $\begin{array}{l}\text { (Lawrance et al, } \\
\text { 2003) }\end{array}$ \\
\hline TIMP-1 & $\begin{array}{lll}\text { anaerobic bacteria and } \\
\text { TNBS/rat }\end{array}$ & ALK5 and Smad proteins mediated TIMP-1 and collagen synthesis. & $\begin{array}{l}\text { (Medina et al, } \\
\text { 2011) }\end{array}$ \\
\hline MMP-2, -7, -9 & DSS/mouse & $\begin{array}{l}\text { Auraptene remarkably suppressed the DSS-induced gelatinolytic } \\
\text { activity of MMP-7 as well as the expression of MMP-2 and }-9 \text {, } \\
\text { suggesting that it might be useful in anti-metastatic therapies via the } \\
\text { targeting of MMPs. }\end{array}$ & $\begin{array}{l}\text { (Kawabata et al, } \\
\text { 2006a) }\end{array}$ \\
\hline
\end{tabular}




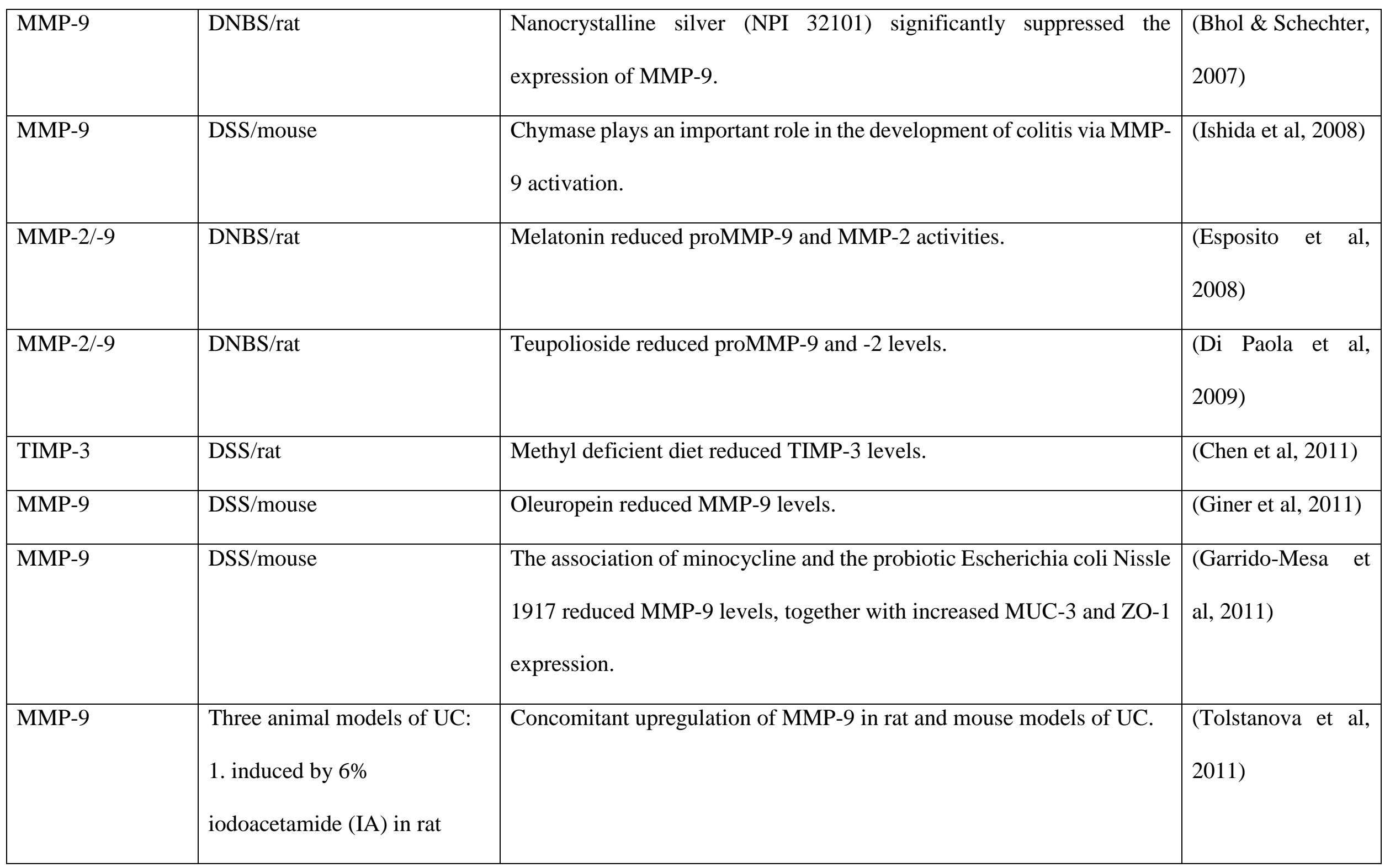




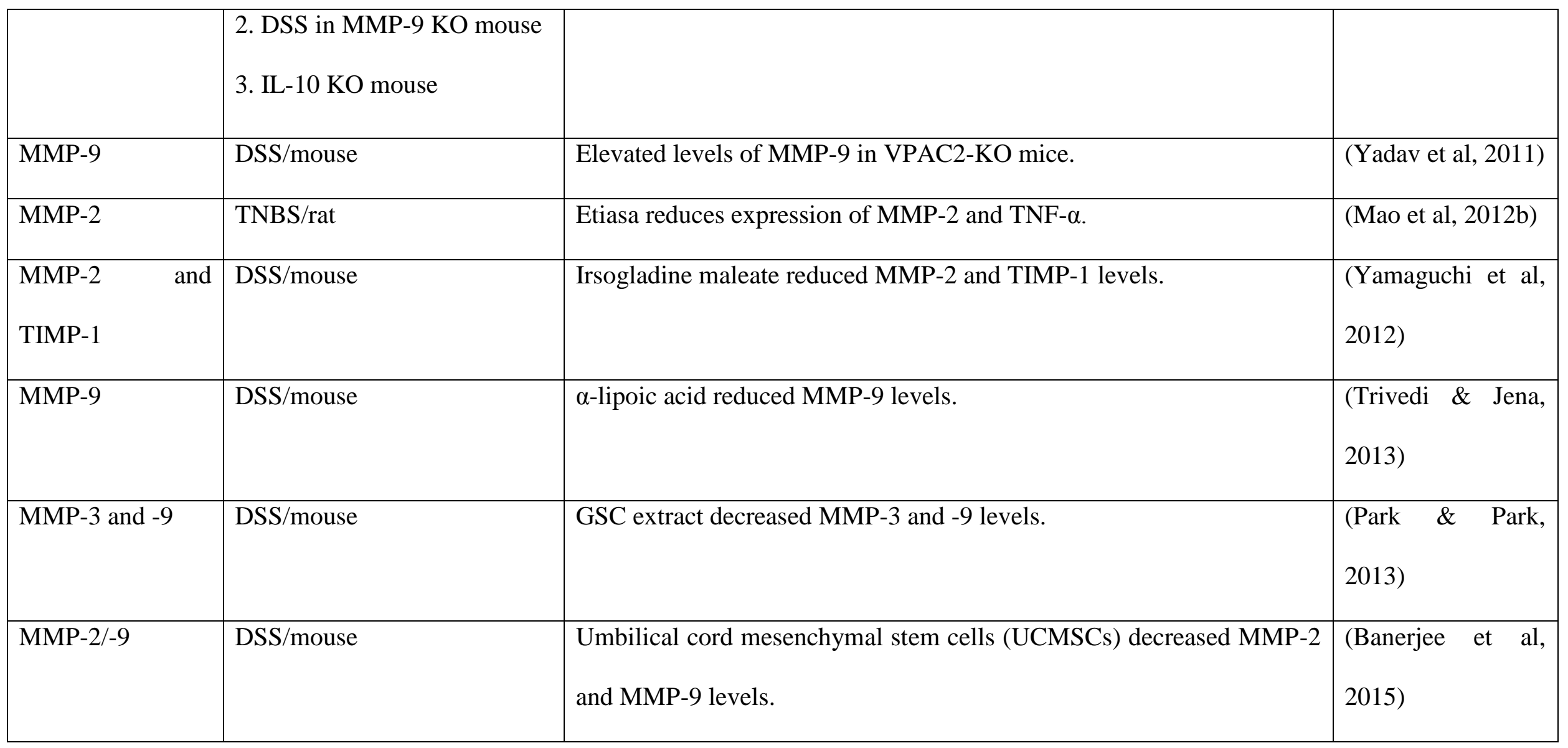


Table 8: Overview of experimental colitis studies in MMP/TIMP deficient mice.

\begin{tabular}{|c|c|c|c|c|}
\hline $\begin{array}{l}\text { MMP/TIMP } \\
\text { deficiency }\end{array}$ & $\begin{array}{l}\text { Genetic } \\
\text { background }\end{array}$ & Animal model & Research conclusions & References \\
\hline \multirow[t]{3}{*}{ MMP-2 -/- } & C57BL/6 & DSS/mouse & More severe DSS-induced colitis. & (Garg et al, 2006) \\
\hline & C57BL/6 & DSS/mouse & Less severe DSS-induced colitis. & $\begin{array}{l}\text { (Heimesaat et al, } \\
\text { 2011b) }\end{array}$ \\
\hline & C57BL/6j & $\begin{array}{l}\text { Campylobacter jejuni } \\
\text { /mouse }\end{array}$ & $\begin{array}{l}\text { Less severe infectious colitis and elevated IL-22, IL- } \\
18, \mathrm{IL}-17 \mathrm{~A} \text {, and IL-1 } \beta \text { levels. }\end{array}$ & (Alutis et al, 2015a) \\
\hline MMP-3 -/- & $\begin{array}{l}\text { NA (Merck } \\
\text { Research } \\
\text { Laboratories) }\end{array}$ & $\begin{array}{l}\text { Citrobacter } \\
\text { rodentium/mouse }\end{array}$ & $\begin{array}{l}\text { MMP-3 plays a role in the migration of } \mathrm{CD}^{+} \mathrm{T} \\
\text { lymphocytes to the intestinal mucosa }\end{array}$ & (Li et al, 2004) \\
\hline MMP-7 -/- & NA & $\begin{array}{l}\text { Salmonella } \\
\text { enterica serovar } \\
\text { Typhimurium } \\
(\text { S.T.)/mouse }\end{array}$ & $\begin{array}{l}\text { More severe infectious colitis. MMP-7 is involved in } \\
\text { host defense against bacteria through activation of } \\
\text { defensins. }\end{array}$ & (Wilson et al, 1999) \\
\hline
\end{tabular}




\begin{tabular}{|c|c|c|c|c|}
\hline & C57BL/6 & DSS/mouse & $\begin{array}{l}\text { More severe colitis. Upregulated IL-1 } 1 \beta \text { levels in } \\
\text { control and DSS-treated MMP-7 KO mice. }\end{array}$ & (Shi et al, 2007) \\
\hline & $\mathrm{C} 57 \mathrm{BL} / 6$ & DSS/mouse & $\begin{array}{l}\text { Less mortality after DSS-induced colitis. Impaired re- } \\
\text { epithelialization and neutrophil infiltration. }\end{array}$ & (Swee et al, 2008) \\
\hline & C57BL6/J & LPS/mouse & $\begin{array}{l}\text { Less severe ileal inflammation and increased } \\
\text { intestinal permeability. }\end{array}$ & $\begin{array}{l}\text { (Vandenbroucke et al, } \\
\text { 2014) }\end{array}$ \\
\hline \multirow[t]{6}{*}{ MMP-9 -/- } & FVB.B6 & DSS/mouse & Attenuated DSS-induced colitis. & (Castaneda et al, 2005) \\
\hline & FVB & DSS/mouse & Attenuated DSS-induced colitis. & (Santana et al, 2006) \\
\hline & $\mathrm{C} 57 \mathrm{BL} / 6$ & NA & $\begin{array}{l}\text { Increased number of goblet cells and MUC-2 } \\
\text { expression. }\end{array}$ & (Garg et al, 2007) \\
\hline & C57BL/6j & Azoxymethane + DSS & $\begin{array}{l}\text { Increased susceptibility to colitis-associated cancer } \\
\text { (CAC). }\end{array}$ & (Garg et al, 2010) \\
\hline & $\mathrm{C} 57 \mathrm{BL} / 6$ & DSS/mouse & No attenuation of colitis. & $\begin{array}{l}\text { (Heimesaat et al, } \\
\text { 2011b) }\end{array}$ \\
\hline & $\begin{array}{l}\text { B6.FVB }(\mathrm{Cg})- \\
\text { mmp9 } 9^{\mathrm{tm} 1 \mathrm{Tvu} / J}\end{array}$ & $\begin{array}{l}\text { Citrobacter } \\
\text { rodentium/mouse }\end{array}$ & $\begin{array}{l}\text { No attenuation of infectious colitis, MMP-9 plays a } \\
\text { role in gut microbiota homeostasis. }\end{array}$ & (Rodrigues et al, 2012) \\
\hline
\end{tabular}




\begin{tabular}{|c|c|c|c|c|}
\hline & C57BL/6j & $\begin{array}{l}\text { Campylobacter jejuni } \\
\text { /mouse }\end{array}$ & $\begin{array}{l}\text { No attenuation of infectious colitis, although lowered } \\
\text { infiltration of immune cells. }\end{array}$ & (Alutis et al, 2015a) \\
\hline & C57BL/6 & DSS/mouse & $\begin{array}{l}\text { Attenuated DSS-induced colitis. MMP-9 modulates } \\
\text { colonic permeability through increased MLCK } \\
\text { expression. }\end{array}$ & (Nighot et al, 2015) \\
\hline MMP-2/-9 -/- & C57BL/6 & $\begin{array}{l}\text { DSS, TNBS and } \\
\text { Salmonella } \\
\text { enterica serovar } \\
\text { Typhimurium } \\
\text { (S.T.)/mouse }\end{array}$ & $\begin{array}{l}\text { Attenuation of colitis. MMP-9 overrides the barrier } \\
\text { protective effect of MMP-2. }\end{array}$ & (Garg et al, 2009) \\
\hline Tg-villin-MMP-9 & C57BL/6 & $\begin{array}{l}\text { DSS and Salmonella } \\
\text { enterica serovar } \\
\text { Typhimurium } \\
\text { (S.T.)/mouse }\end{array}$ & $\begin{array}{l}\text { More severe colitis. MMP-9 is associated with } \\
\text { increased levels of proinflammatory cytokine Kc. }\end{array}$ & (Liu et al, 2013) \\
\hline $\begin{array}{l}\text { MMP-9 } \\
\text { overexpression }\end{array}$ & C57BL/6 & $\begin{array}{l}\text { exogenous MMP- } \\
\text { 9/mouse }\end{array}$ & $\begin{array}{l}\text { More severe colitis. MMP-9 causes increased } \\
\text { intestinal epithelial tight junction permeability. }\end{array}$ & (Nighot et al, 2015) \\
\hline
\end{tabular}




\begin{tabular}{|c|c|c|c|c|}
\hline Mmp-10 -/- & $\mathrm{C} 57 \mathrm{BL} / 6$ & DSS/mouse & $\begin{array}{l}\text { More severe colitis and development of dysplastic } \\
\text { lesions in the colon. }\end{array}$ & (Koller et al, 2012) \\
\hline MMP-12 -/- & C57BL/6 & TNBS/mouse & Less severe colitis. & (Pender et al, 2006) \\
\hline MMP-13 -/- & $\mathrm{C} 57 \mathrm{BL} / 6$ & DSS/mouse & $\begin{array}{l}\text { Less severe colitis. MMP13 contributes to intestinal } \\
\text { permeability via TNF shedding. }\end{array}$ & $\begin{array}{l}\text { (Vandenbroucke et al, } \\
\text { 2013) }\end{array}$ \\
\hline MMP-19 -/- & $\mathrm{C} 57 \mathrm{BL} / 6$ & DSS/mouse & $\begin{array}{l}\text { More severe colitis. MMP-19 is important in innate } \\
\text { immune response in colitis. }\end{array}$ & (Brauer et al, 2015) \\
\hline TIMP-3 -/- & $\mathrm{C} 57 \mathrm{BL} / 6$ & $\begin{array}{l}\text { TNBS and T cell } \\
\text { transfer/mouse }\end{array}$ & $\begin{array}{l}\text { Loss or reduction of TIMP-3 in mice promotes } \\
\text { development of colitis. }\end{array}$ & $\begin{array}{l}\text { (Monteleone et al, } \\
\text { 2012) }\end{array}$ \\
\hline Tg-CD68-TIMP-3 & $\mathrm{C} 57 \mathrm{BL} / 6$ & TNBS/mouse & $\begin{array}{l}\text { TIMP-3-Tg mice were resistant to TNBS-induced } \\
\text { colitis. }\end{array}$ & $\begin{array}{l}\text { (Monteleone et al, } \\
\text { 2012) }\end{array}$ \\
\hline TIMP-1 -/- & $\mathrm{C} 57 \mathrm{BL} / 6$ & DSS/mouse & $\begin{array}{l}\text { Paradoxical development of acute colitis, protected } \\
\text { from chronic colitis and fibrosis. }\end{array}$ & $\begin{array}{l}\text { (Breynaert \& de Bruyn } \\
\text { et al., in press) }\end{array}$ \\
\hline
\end{tabular}


Table 9: Overview of MMP inhibitors in IBD animal models.

\begin{tabular}{|c|c|c|c|}
\hline MMP inhibitor & IBD & Research conclusions & References \\
\hline Marimastat & TNBS/rat & Reduced tissue injury and inflammation. & (Sykes et al, 1999) \\
\hline Batimastat & TNBS/rat & Reduced colonic inflammation and MPO activity. & $\begin{array}{l}\text { (Di Sebastiano et al, } \\
\text { 2001) }\end{array}$ \\
\hline CGS-27023-A & DSS/rat & $\begin{array}{l}\text { Decreased extent and severity of epithelial injury but no influence } \\
\text { on mucosal repair. }\end{array}$ & $\begin{array}{l}\text { (Medina et al, } \\
\text { 2003) }\end{array}$ \\
\hline ONO-4817 & DSS/mouse & $\begin{array}{l}\text { Reduced shortening of the colon, infiltration of inflammatory cells } \\
\text { and MPO activity. }\end{array}$ & (Naito et al, 2004) \\
\hline CGS-27023-A & TNBS/rat & MMP-9 modulates intestinal injury in rats with transmural colitis. & $\begin{array}{l}\text { (Medina et al, } \\
\text { 2006a) }\end{array}$ \\
\hline MMP3-specific siRNA & DSS/mouse & Improved clinical score. & $\begin{array}{l}\text { (Kobayashi et al, } \\
\text { 2006) }\end{array}$ \\
\hline MMP10-specific siRNA & DSS/mouse & Improved clinical score. & $\begin{array}{l}\text { (Kobayashi et al, } \\
\text { 2006) }\end{array}$ \\
\hline
\end{tabular}




\begin{tabular}{|c|c|c|c|}
\hline Ilomastat & TNBS/rat & Reduced inflammation through inhibition of MMP-1. & $\begin{array}{l}\text { (Wang \& Wang, } \\
\text { 2008) }\end{array}$ \\
\hline \multirow[t]{2}{*}{ Minocycline } & TNBS/mouse & $\begin{array}{l}\text { Decreased mortality rate and inhibited pro-inflammatory cytokine } \\
\text { expression in colonic tissues. }\end{array}$ & (Huang et al, 2009) \\
\hline & DSS/mouse & $\begin{array}{l}\text { Reduced body weight loss, improved colonic histology and } \\
\text { blocked expression of iNOS, pro-inflammatory cytokines and } \\
\text { MMPs from colonic tissues. }\end{array}$ & (Huang et al, 2009) \\
\hline $\begin{array}{l}\text { synthetic collagen analogue (Gly-Pro- } \\
\text { Hyp) } 10\end{array}$ & DSS/mouse & $\begin{array}{l}\text { Ameliorated clinical symptoms and histopathological colonic } \\
\text { changes. }\end{array}$ & $\begin{array}{l}\text { (Heimesaat et al, } \\
\text { 2012) }\end{array}$ \\
\hline RO28-2653 & DSS/mouse & $\begin{array}{l}\text { Ameliorated clinical colitis pathology. Lack of Escherichia coli } \\
\text { overgrowth. }\end{array}$ & $\begin{array}{l}\text { (Heimesaat et al, } \\
\text { 2011a) }\end{array}$ \\
\hline AE-941 & TNBS/rat & Reduced colonic mucosal damage. & (Mao et al, 2012a) \\
\hline SDS3 & DSS/mouse & $\begin{array}{l}\text { Attenuation of DSS-induced colitis. Directed against the activated } \\
\text { forms of MMP-2 and MMP-9. }\end{array}$ & $\begin{array}{l}\text { (Sela-Passwell et al, } \\
\text { 2012) }\end{array}$ \\
\hline PGP antagonist & DSS/mouse & $\begin{array}{l}\text { Significant reduction in neutrophil infiltration in the intestine. } \\
\text { AB0041 inhibited release of sTNF- } \alpha \text { by MMP-9. }\end{array}$ & $\begin{array}{l}\text { (Koelink et al, } \\
2014)\end{array}$ \\
\hline
\end{tabular}




\begin{tabular}{|l|l|l|l|}
\hline MMP-9 antagonist & DSS/mouse & Reduced severity of colitis and reduced tumor growth and \\
& & metastases incidence. & 2015) \\
\hline
\end{tabular}




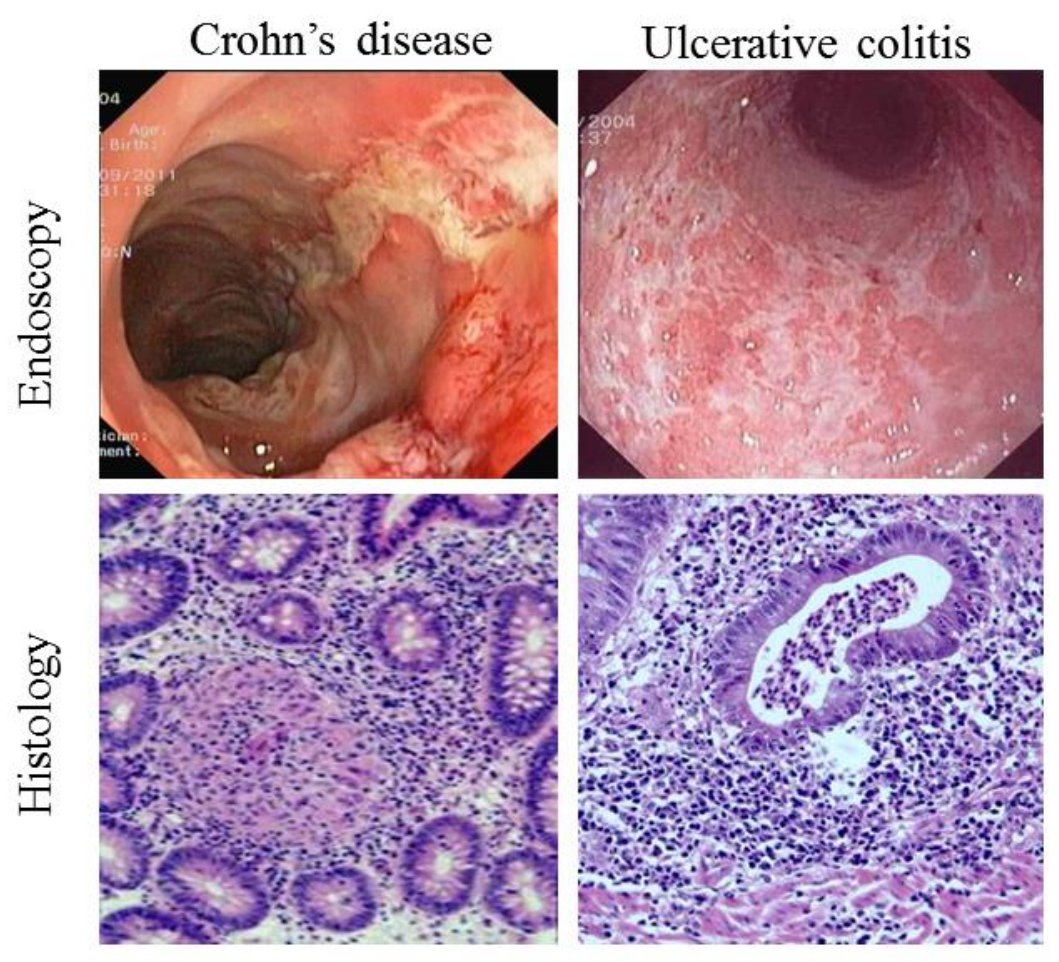

Figure 1: Endoscopic and histological features of Crohn's disease (CD) and ulcerative colitis (UC). CD is characterized endoscopically by linear and serpinguous ulcerations and patchy inflammation ultimately resulting in a cobblestone pattern, whereas histologically an infiltration of inflammatory cells and presence of granuloma can be visualized. In UC, erythema, loss of vascular pattern, friability and erosions can be seen during an endoscopic evaluation, whereas histological inflammation is characterized by infiltration of inflammatory cells, goblet cell loss and crypt abscesses. 
A

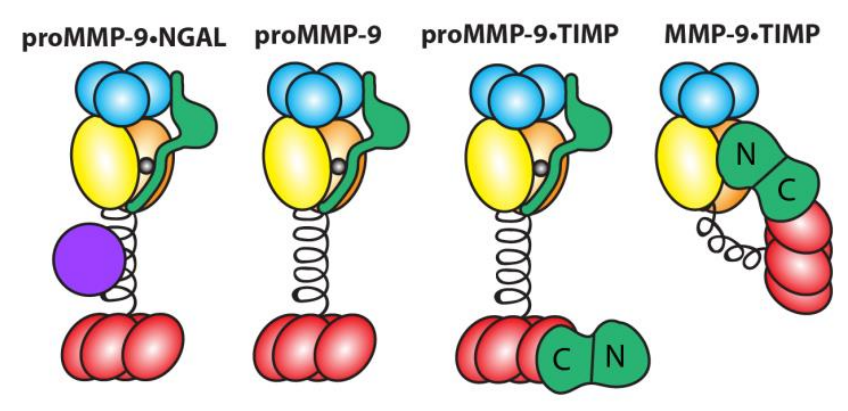

B

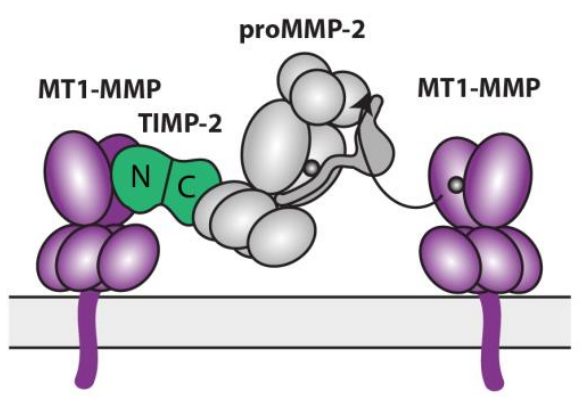

C

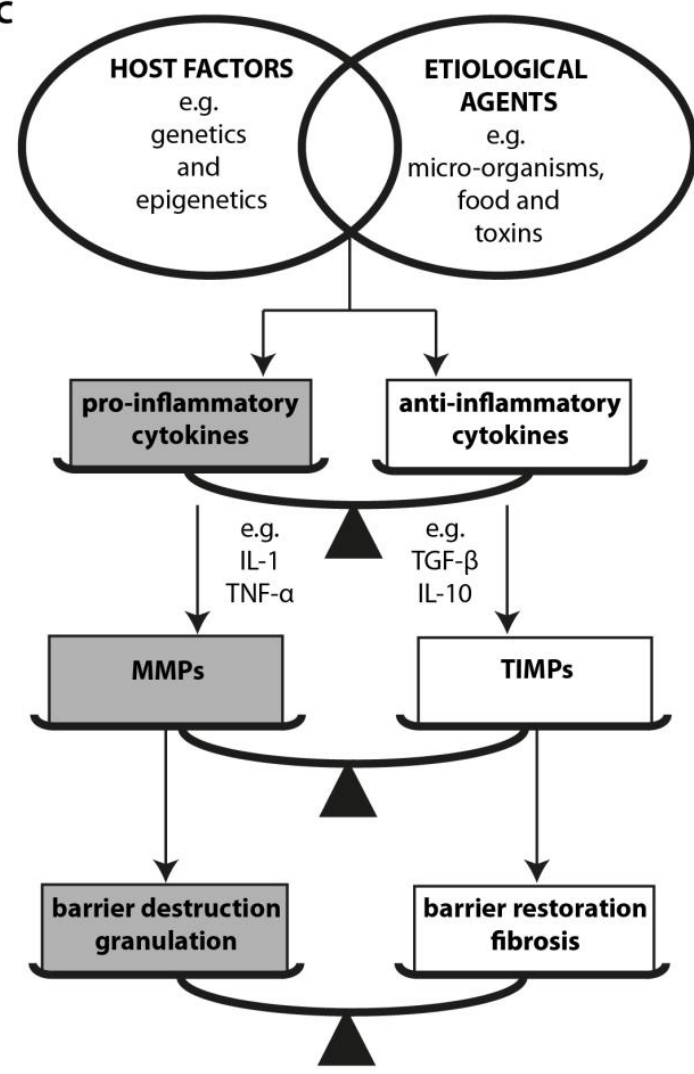

Figure 2: Interactions and balances between MMPs and TIMPs. A. MMPs are multidomain molecules, here exemplified by proMMP-9. The propeptide (in green) keeps newly synthesized MMPs inactive. Together, the active site (in yellow) and zinc (grey atom) binding domain (in orange) form the active center of MMP proteolysis. The C-terminal haemopexin domain (in red) is involved in substrate binding and is present in several MMPs. The three fibronectin repeats (in blue) are only present in MMP-2/gelatinase A and MMP-9/gelatinase B and aid the degradation of gelatins. Uniquely, MMP-9 also contains a highly flexible O-glycosylated domain (in black). The C-terminal domain of TIMPs (in green) can interact with the haemopexin domain whereas the N-terminal domain can inhibit MMP catalysis by interaction with the active site zinc molecule. Whereas single interaction sites between TIMPs and MMPs have been characterized, the interaction of TIMPs and MMPs in macromolecular complexes such as NGAL-MMP-9 remains elusive. B. TIMPs assist in the activation of proMMPs. For example, proMMP-2 activation involves the formation of an MT1-MMP, TIMP-2, proMMP-2 
complex at the cell surface. A second MT1-MMP molecule then removes the propeptide of the tethered pro-MMP-2 (Adapted from (Itoh \& Seiki, 2004)). C. In physiological conditions a delicate balance exists between MMPs and TIMPs. In IBD excess activity of MMPs leads to destruction of the intestinal barrier, generates remnant epitopes from intact proteins and results in granulation tissue. At a higher level the MMP/TIMP balances are regulated by balances between pro-inflammatory and anti-inflammatory cytokines and the production of cytokines is under the control of host and environmental factors (Adapted from (Opdenakker \& Van Damme, 1994) and (Opdenakker \& Van Damme, 2011). Excess TIMP might restore the intestinal barrier and can lead to fibrosis. 


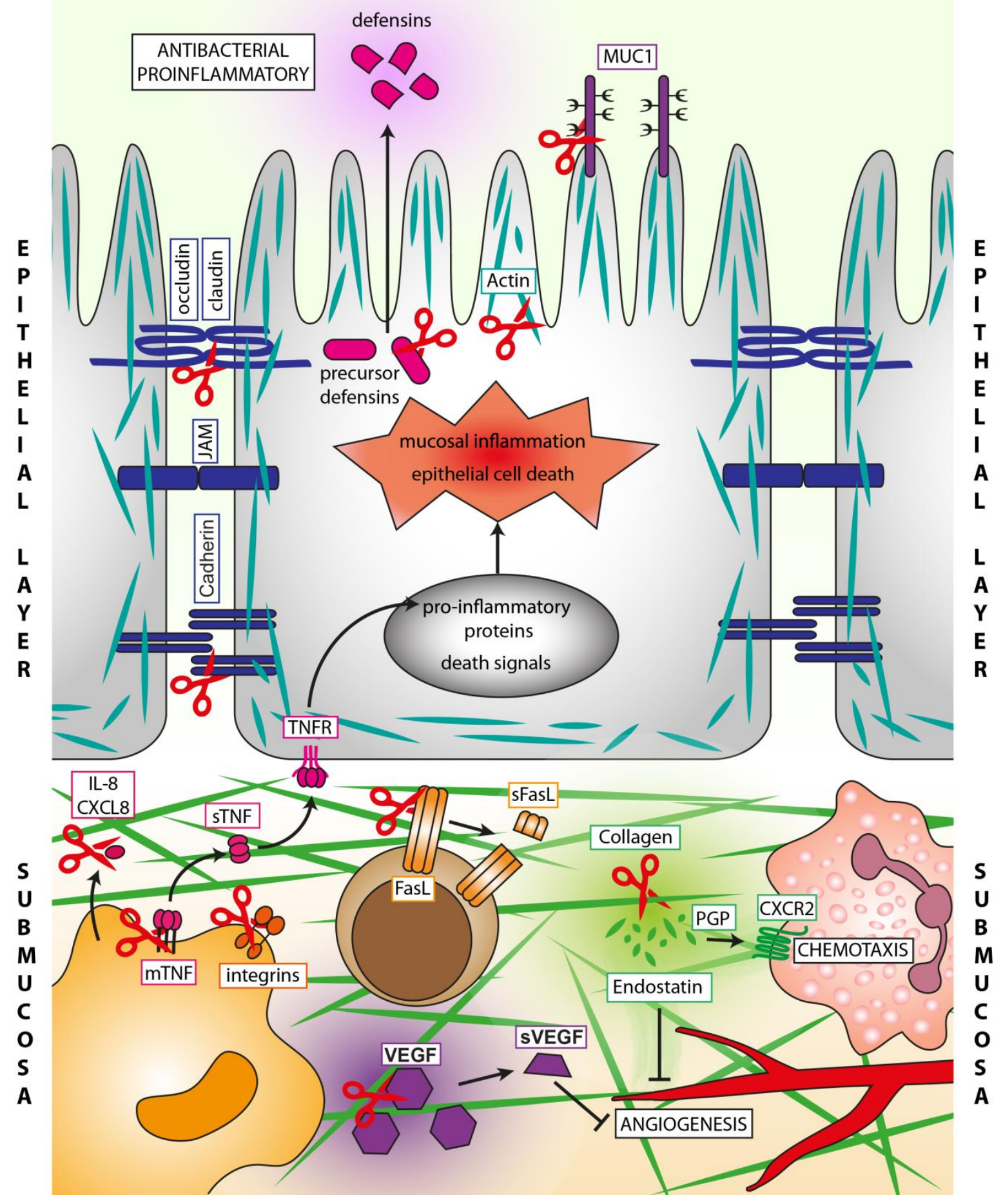

Figure 3: MMP substrates with potential roles in IBD. MMPs have a vast range of extracellular, pericellular and intracellular substrates. In the intestinal lumen, antibacterial molecules such as the membrane bound mucin-1 (MUC1) and secreted defensins can be modified by MMPs thereby altering the host-bacterial interaction and contributing to inflammation. Within the epithelial layer, MMPs can degrade intercellular junction molecules 
(e.g. cadherins, occludins and claudins) and intracellular structural proteins (e.g. actins), leading to altered cell shapes and barrier disruption. The degradation of extracellular matrix (ECM) in the submucosa (e.g. collagens) results in the release of chemotactic (PGP) and anti-angiogenic (endostatin) collagen fragments. Moreover, MMPs can release ECM-bound VEGF (sVEGF) which can change angiogenic processes. Finally, immune cells such as macrophages (in orange), lymphocytes (in brown) and neutrophils (in pink) contain and secrete many MMP substrates such as cytokines (e.g. TNF), chemokines (e.g. IL-8/CXCL8), adhesion molecules (e.g. integrins) and survival molecules (e.g. FasL). 


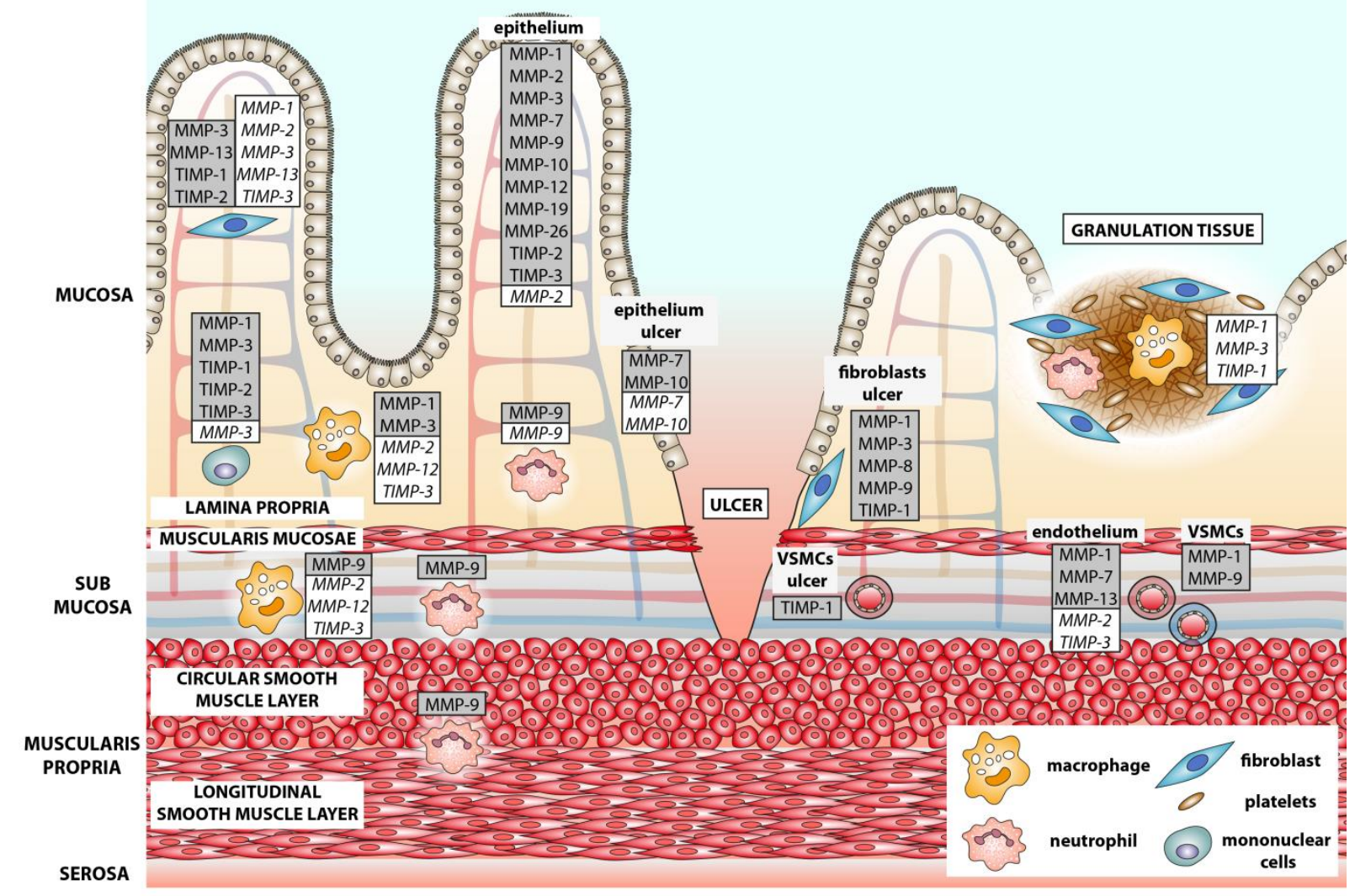

Figure 4: Overview of the cellular localization of MMPs and TIMPs in the intestinal

layers. MMPs and TIMPs are mostly studied in the mucosa, but are also present in submucosa and muscularis propria. The healthy epithelium expresses a wide range of MMPs and TIMPs, but, in injured epithelium, MMP-7 and MMP-10 are more pronounced which suggests a role for MMP-7 and MMP-10 in epithelial regeneration (Vaalamo et al, 1998). Stromal cells such as fibroblasts also express many MMPs and TIMPs, but fibroblasts containing MMP-1, $-3,-8$ and -9 have been associated with areas of inflammation at the base of ulcers (Arihiro et al, 2001). Immune cells also contribute to MMP and TIMP expression in mucosal and submucosal layers. Neutrophils, in particular, abundantly express MMP-9 (Opdenakker et al, 2001b). Whereas TIMPs are expressed across different cell types, TIMP-3 is mainly associated with healthy intestine and is less abundant in inflamed intestine (Vaalamo et al, 1998). RNA expression is shown on a white background and protein expression on a gray background. 


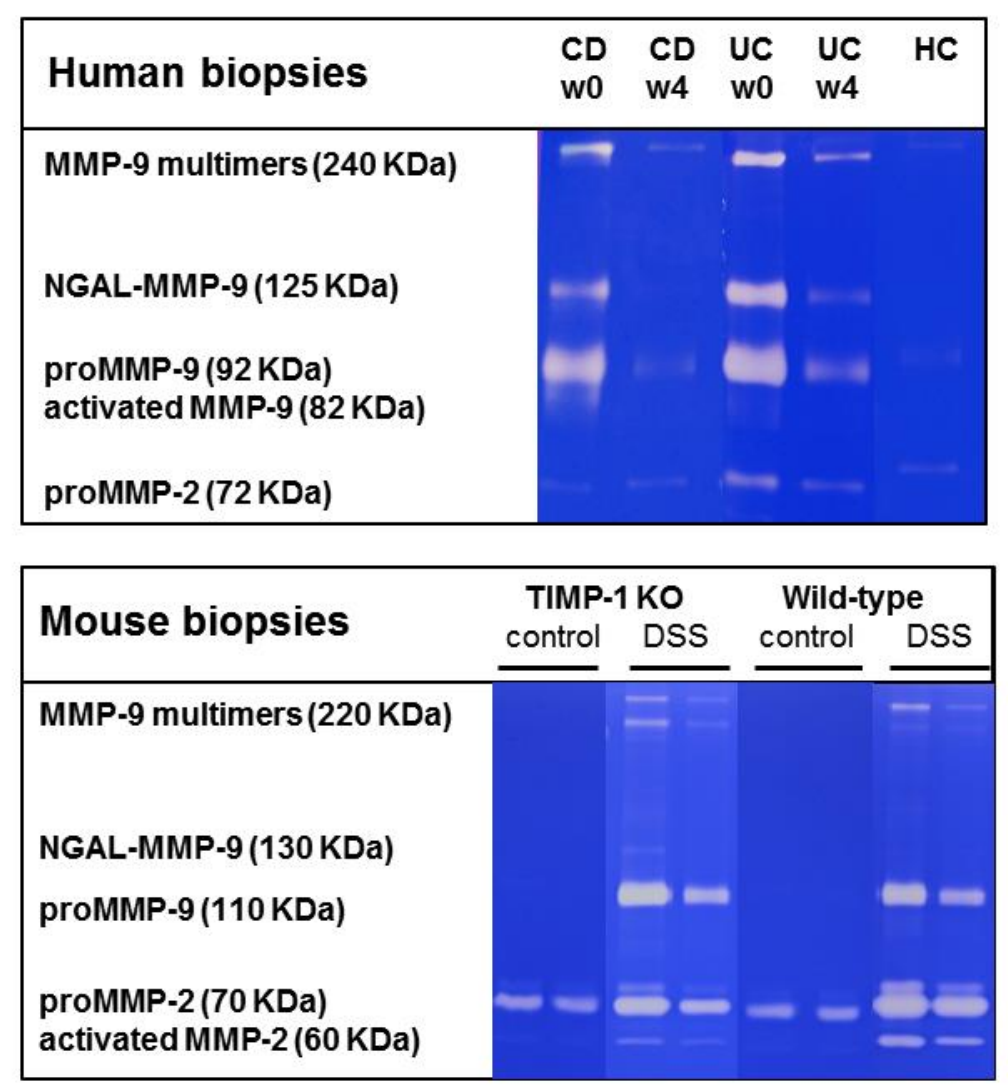

Figure 5. Gelatin zymography template illustrating several forms of gelatinases $A$ and $B$ present in human- and mouse-derived intestinal tissues. In the top panel, zymography profiles from mucosal biopsies derived from IBD (CD and UC, respectively) patients are shown before (w0) and after (w4) treatment with infliximab as well as from one healthy control (HC) individual. In the lower panel, zymography profiles are shown from homogenized colonic tissues isolated from TIMP-1 knockout (KO) and wild-type mice in control conditions and after dextran sodium sulphate (DSS) administration. MMP-9 multimers, NGAL-MMP-9 complex, proMMP-9, activated MMP-9, proMMP-2 and activated MMP-2 forms can be distinguished in both human and mouse intestinal tissues. However, NGAL-MMP-9 levels were less frequently observed in mouse tissues. Moreover, mouse and human gelatinase forms have distinct molecular weights (KDa). 